\title{
A Study of Atmospheric Neutrino Oscillations in the MINOS Far Detector
}

\author{
Andrew Blake \\ St. John's College
}

A dissertation submitted to the University of Cambridge

for the degree of Doctor of Philosophy

August 2005 


\title{
A Study of Atmospheric Neutrino Oscillations in the MINOS Far Detector
}

\author{
Andrew Blake
}

\begin{abstract}
In recent years, neutrino experiments have begun to challenge the Standard Model assumption that neutrinos are massless. There is now firm evidence that neutrinos undergo quantum mechanical oscillations between flavours. This would imply that neutrinos possess mass and that neutrino flavours are mixed by the weak interaction. Atmospheric neutrinos, produced by the interactions of cosmic rays in the earth's atmosphere, can be used to study these oscillations.

The MINOS Far Detector has been collecting atmospheric neutrino data since 1st August 2003 using a $5.4 \mathrm{kT}$ steel-scintillator sampling calorimeter located $700 \mathrm{~m}$ underground (2100 m water-equivalent) at the Soudan Underground Laboratory, Minnesota. The Far Detector is the first massive underground detector to possess a magnetic field. This makes the separation of atmospheric $\nu_{\mu}$ and $\bar{\nu}_{\mu}$ charged current interactions possible for the first time.

This thesis presents a study of atmospheric neutrino oscillations in the Far Detector, based on a total detector exposure of 316 days (3.3 kT-Yrs fiducial exposure). The separation of atmospheric neutrinos from the high background of cosmic muons is outlined. A total of 82 candidate events are observed, with an expectation of $109.9 \pm 21.4$ events in the absence of oscillations. Of the selected events, 40 events have a clcarly identified charge, with 27 events tagged as neutrinos and 13 events tagged as anti-neutrinos. This represents the first direct observation of atmospheric $\nu_{\mu}$ and $\bar{\nu}_{\mu}$ charged current interactions. A maximum likelihood analysis is used to determine the allowed region for the oscillation parameters $\Delta m_{23}^{2}$ and $\sin ^{2} 2 \theta_{23}$. This disfavours the null oscillation hypothesis at the $79 \%$ confidence level. With the current low statistics, the sensitivity of the analysis is limited. The expected future sensitivity of the atmospheric neutrino analysis is discussed.
\end{abstract}




\section{Declaration}

This dissertation is my own work except where explicit reference is made to the work of others, and has not been submitted for another qualification to this or any other university.

Andrew Blake 


\section{Acknowledgements}

I am grateful for the help and support of many people. First and foremost, I would like to thank my supervisor, Mark Thomson, for his exceptional advice and guidance throughout my PhD. I have been fortunate to have the opportunity of working with such a good physicist and nice guy. There are several others who have made important contributions to the work presented in this thesis. Caius Howcroft has played an instrumental role in developing the analysis and I am indebted to his expert knowledge and valucd friendship. I have also received a great deal of help and support from Pat Ward, David Ward, John Chapman, Andy Culling, John Marshall and Matt Palmer. Over the past four years, I have had the pleasure of working with many people in many places. I would like to take this opportunity to thank all those who have helped to make my PhD a great experience. Finally, I would like to thank my family and friends for all their support and encouragement - I couldn't have done it without you! 
In memory of Shirley Astbury 
We shall not cease from exploration And the end of our exploring Will be to arrive where we started And know the place for the first time. T. S. Eliot - Littlc Gidding 


\section{Contents}

1 Introduction $\quad 1$

2 Neutrino Oscillations $\mathbf{5}$

2.1 Overview . . . . . . . . . . . . . . . . . . . .

2.2 Theory of Neutrino Oscillations . . . . . . . . . . . . . . 6

2.3 Neutrino Oscillation Experiments . . . . . . . . . . . . . . . 10

2.3.1 Solar Neutrinos . . . . . . . . . . . . . . . . 10

2.3 .2 SNO . . . . . . . . . . . . . . . . . . . 15

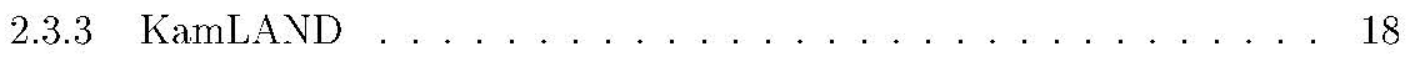

2.3.4 Atmospheric Neutrinos . . . . . . . . . . . . . . . . . . 20

2.3.j Super-Kamiokande . . . . . . . . . . . . . . 23

$2.3 .6 \mathrm{~K} 2 \mathrm{~K} \ldots \ldots \ldots \ldots \ldots \ldots \ldots$

$2.3 .7 \mathrm{CHOOZ} \ldots \ldots \ldots \ldots \ldots \ldots \ldots$

2.3 .8 LSND . . . . . . . . . . . . . . . . . . . . . . . 28

2.4 The Current Status . . . . . . . . . . . . . . . . . . . . 31

3 The MINOS Experiment $\quad 33$

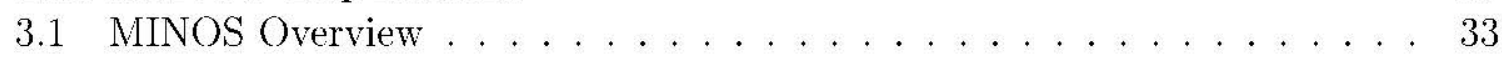

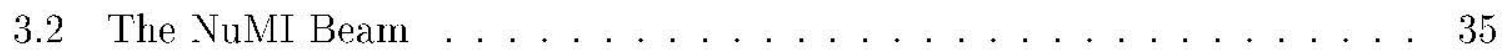

3.3 The MINOS Detectors . . . . . . . . . . . . . . . . . . . 39

3.4 Beam Neutrino Physics Analysis . . . . . . . . . . . . . . . . . . . 40

$3.4 .1 \nu_{\mu} \rightarrow \nu_{\tau}$ disappcarance scarch . . . . . . . . . . . . . 42

$3.4 .2 \nu_{\mu} \rightarrow \nu_{e}$ appearance search . . . . . . . . . . . . . 42

3.5 Atmospheric Neutrino Physics Analysis . . . . . . . . . . . . . . . . . 45

4 The MINOS Far Detector $\quad 47$

4.1 Detector Overview . . . . . . . . . . . . . . . . . . . . . . . . . . . . . 47

4.2 Detector Technologies . . . . . . . . . . . . . . . . . 48

4.2 .1 Plastic Scintillator . . . . . . . . . . . . . . . 48

4.2.2 M16 Photo-multiplier Tubes . . . . . . . . . . . . . . 51

4.2 .3 Front-End Electronics . . . . . . . . . . . . . . . . . . . . 04

4.2.4 Data Acquisition + Trigger Processing . . . . . . . . . . . . j6

4.2.5 Detector Control Systern . . . . . . . . . . . . . . . . 57

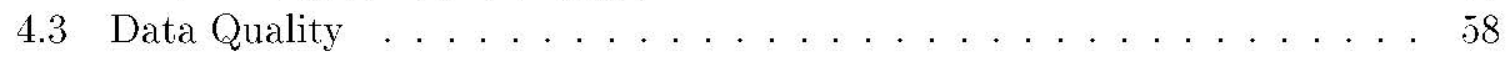


4.3 .1 Coil Currents . . . . . . . . . . . . . . . . . . . 58

4.3 .2 Dead Electronics . . . . . . . . . . . . . . . . . 60

4.3 .3 Busy Electronics . . . . . . . . . . . . . . . . 60

4.4 The Veto Shield . . . . . . . . . . . . . . . . . . 62

5 Detector Calibration $\quad 67$

5.1 Calibration Overview . . . . . . . . . . . . . . . 67

5.2 Timing Calibration . . . . . . . . . . . . . . . . . . 68

5.2 .1 Calibration Procedure . . . . . . . . . . . . . 70

5.2 .2 Mcasuring the Refractive Index . . . . . . . . . . . . . 72

5.2 .3 Hardware Changes . . . . . . . . . . . . . . . . . 73

5.2 .4 Time Walk Corrections . . . . . . . . . . . . . 76

5.2 .5 Strip-to-Strip Calibration . . . . . . . . . . . . 77

5.2 .6 Validating the Calibration . . . . . . . . . . . . . 79

5.3 Pulse Height Calibration . . . . . . . . . . . . . . . . . . . . 82

5.3 .1 Gain Calibration . . . . . . . . . . . . . . . 84

5.3 .2 Linearity Calibration . . . . . . . . . . . . . . . . 84

5.3 .3 Strip-to-Strip Calibration . . . . . . . . . . . . 86

5.3 .4 Energy Calibration . . . . . . . . . . . . . . 87

6 Monte Carlo Simulation $\quad 89$

6.1 Simulation Overview . . . . . . . . . . . . . . . . . . . . . . . . . 89

6.2 Detector Simulation . . . . . . . . . . . . . . . . . . 90

6.2.1 Simulation of Active Detector . . . . . . . . . . . . 90

6.2.2 Simulation of M16 PMTs and VA Electronics . . . . . . . . . 91

6.2.3 Data-MC Comparison of Detector Response . . . . . . . . . . 91

6.3 Cosmic Muon Simulation . . . . . . . . . . . . . . . . . . . . . 95

6.3.1 The Cosmic Ray Flux . . . . . . . . . . . . . . . . . 95

6.3.2 Simulation of Underground Cosmic Muon Flux . . . . . . . . 98

6.3.3 Data-MC Comparison of Cosmic Muon Flux . . . . . . . . . . . 99

6.4 Atmospheric Neutrino Simulation . . . . . . . . . . . . . . 102

6.4.1 Simulation of Atmospheric Neutrino Flux . . . . . . . . . . . 102

6.4.2 Simulation of Atmospheric Neutrino Cross-Sections . . . . . . . 106

6.5 Monte Carlo Samples . . . . . . . . . . . . . . . . . . . . . 108

7 Event Reconstruction $\quad 111$

7.1 Reconstruction Overvicw . . . . . . . . . . . . . . . . . . . 111

7.2 Low Level Reconstruction . . . . . . . . . . . . . . . . . . . . . . 113

7.3 Reconstruction of Particle Tracks and Showers . . . . . . . . . . . 117

7.4 Reconstruction of Event Kinematics . . . . . . . . . . . . . . . . 126

7.4.1 Direction Reconstruction . . . . . . . . . . . . . . . . 126

7.4 .2 Charge Reconstruction . . . . . . . . . . . . . . . 133

7.4 .3 Energy Reconstruction . . . . . . . . . . . . . . 137

8 Selection of Atmospheric Neutrino Events 145 
8.1 Event Selection Overview . . . . . . . . . . . . . . . . . 145

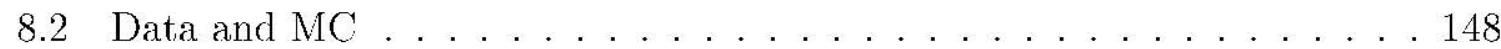

8.3 Low Level Filters . . . . . . . . . . . . . . . . . . . . . . 150

8.3 .1 Hardware Filter . . . . . . . . . . . . . . . 150

8.3 .2 Noise Filter . . . . . . . . . . . . . . . . . . . 151

8.3.3 Containment Filter . . . . . . . . . . . . . . . . . 153

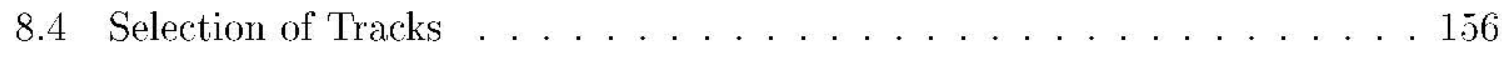

8.5 Selection of FC and Down-Going PC Events . . . . . . . . . . . . . . . . . . . . . . . . . . . . . . .

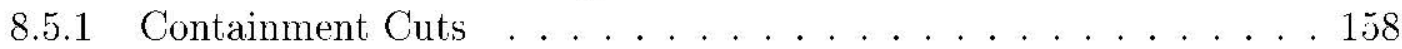

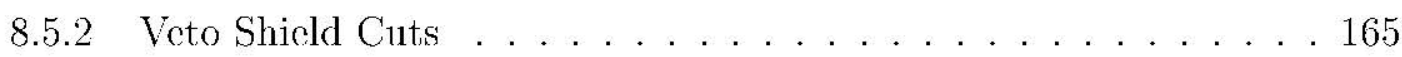

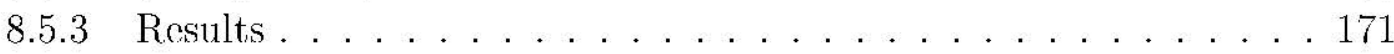

8.6 Sclection of Up-Going PC Events . . . . . . . . . . . . . . . . . . 173

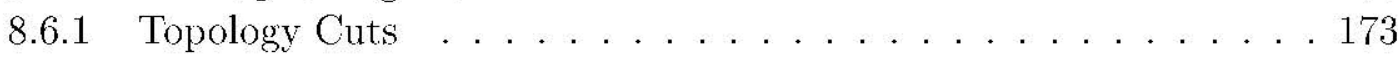

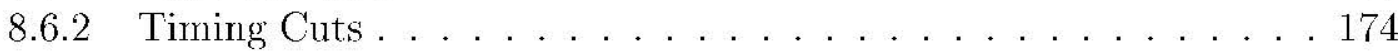

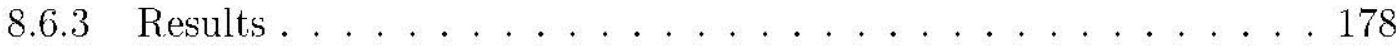

8.7 Selection Efficiencies . . . . . . . . . . . . . . . . . . . . . . . . . . . . . . . . . . . . . . . . . . . .

8.8 Other Backgrounds . . . . . . . . . . . . . . . . . . . . . . . . . . . . . . . . . . . . . . . . . . . .

8.8 .1 Nentrons. . . . . . . . . . . . . . . . . 182

8.8.2 Neutrino-Induced Lp-Going Muons . . . . . . . . . . . . . . . 182

8.8.3 Tau Ncutrinos . . . . . . . . . . . . . . . . . 183

8.9 Systematic Uncertainties in Event Selection . . . . . . . . . . . . . . 184

8.10 Selected Events . . . . . . . . . . . . . . . . 186

9 Neutrino Oscillation Analysis 191

9.1 Analysis Overview . . . . . . . . . . . . . . . . . . . . . . . 191

9.2 Selection of High Resolution Events . . . . . . . . . . . . . . . . . . . . 192

9.2.1 Determination of $u p /$ down Ratio . . . . . . . . . . . . . . 198

9.2.2 Determination of $\bar{\nu}_{\mu} / \nu_{\mu}$ Ratio . . . . . . . . . . . . . . . 199

9.3 Fits to Neutrino Oscillations . . . . . . . . . . . . . . . . . 202

9.3.1 Combined $\nu_{\mu} / \bar{\nu}_{\mu}$ Oscillation Fit . . . . . . . . . . . . . . 209

9.3.2 Separated $\nu_{\mu} / \bar{\nu}_{\mu}$ Oscillation Fit . . . . . . . . . . . . 213

9.4 Projected 25 kT-Yr Sensitivity . . . . . . . . . . . . . . 216

10 Conclusion $\quad 221$

A Atmospheric Neutrino Events $\quad 225$

A.1 Fully Contained Events . . . . . . . . . . . . . . . . . . . . 226

A.2 Down-Going Partially Contained Events . . . . . . . . . . . . . . 228

A.3 Lp-Going Partially Contained Events . . . . . . . . . . . . . . . 229 


\section{List of Figures}

2.1 The p-p fusion chain . . . . . . . . . . . . . . . . . . . . 11

2.2 The Super-Kamiokande detector. . . . . . . . . . . . . . . . . . . . 14

2.3 Super-Kamiokande measurement of neutrino-electron scattering. . . . . . 14

2.4 The S.NO detector. . . . . . . . . . . . . . . . . . . . . . . 16

2.5 SNO measurement of solar neutrino flux. . . . . . . . . . . . . 17

2.6 SNO neutrino oscillation confidence limits . . . . . . . . . . . . . 17

2.7 KamLA.ND measurement of reactor neutrino spectrum _ . . . . . . . 19

2.8 KamLA.VD neutrino oscillation confidence limits . . . . . . . . . . . . . . 19

2.9 Atmospheric neutrino production . . . . . . . . . . . . . . . 21

2.10 Super-Kamiokande atmospheric neutrino zenith angle distribution. . . . . 24

2.11 Super-Kamiokande neutrino oscillation confidence limits . . . . . . . 24

2.12 Super-Kamiokande neutrino oscillation L/E analysis . . . . . . . . . . 25

2.13 K2K measurement of beam spectrum . . . . . . . . . . . . . . 27

$2.14 \mathrm{~K} 2 \mathrm{~K}$ neutrino oscillation confidence limits . . . . . . . . . . . 27

$2.15 \mathrm{CHOOZ}$ measurement of reactor neutrino spectrum . . . . . . . . . 29

$2.16 \mathrm{CHOOZ}$ neutrino oscillation confidence limits . . . . . . . . . . . 29

2.17 The LSND signal . . . . . . . . . . . . . . . . . . . . . . 30

3.1 The MINOS neutrino baseline . . . . . . . . . . . . . . . . . 34

3.2 The NLYI facility . . . . . . . . . . . . . . . . 36

3.3 NuMI focusing horn . . . . . . . . . . . . . . . . 37

3.4 The NuMI beam configurations . . . . . . . . . . . . . . . 38

3.5 The MINOS Near Detector . . . . . . . . . . . . . . . . . . . . 41

3.6 The MINOS Far Detector . . . . . . . . . . . . . . . . . 41

3.7 MINOS sensitivity to $\nu_{\mu} \rightarrow \nu_{\tau}$ oscillations . . . . . . . . . 43

3.8 MINOS scnsitivity to $\nu_{\mu} \rightarrow \nu_{e}$ oscillations . . . . . . . . . 44

4.1 The MINOS Far Detector. . . . . . . . . . . . . . . . . . . . . . . . . 49

4.2 Far Detector scintillator plane. . . . . . . . . . . . . . . . . 50

4.3 Far Detector optical readout. . . . . . . . . . . . . . . . . . 52

4.4 Far Detector front-end readout scheme. . . . . . . . . . . . 55

4.5 Far Detector coil currents . . . . . . . . . . . . . . . . . . . 59

4.6 Far Detector HV trips . . . . . . . . . . . . . . . . . . . . . . 59

4.7 Rates of dead VA chips . . . . . . . . . . . . . . . . 61

4.8 Rates of busy VA chips . . . . . . . . . . . . . . . . . . 61 
4.9 Example of vetoed cosmic muon event. . . . . . . . . . . . . . . . 64

4.10 Veto shicld tagging efficiencies . . . . . . . . . . . . . . 65

5.1 Timing calibration procedurc. . . . . . . . . . . . . . 70

5.2 Measuring the refractive index of WLS fibre (1) . . . . . . . . . . . . . . . . . . . . 74

5.3 Measuring the refractive index of WLS fibre (2) . . . . . . . . . . . . 74

5.4 Example of timing shift due to hardware change. . . . . . . . . . . . . 75

5.5 Chronology of time jumps due to hardware changes. . . . . . . . . . . 75

5.6 Time walk dclay. . . . . . . . . . . . . . . . . . . . . . . . . . . . . . . . . . . . . 78

5.7 Timing calibration constants. . . . . . . . . . . . . . . . 78

5.8 Validation of calibration (1) . . . . . . . . . . . . . . . . . . . . . . . . . . . . 80

5.9 Validation of calibration (2) . . . . . . . . . . . . . . . . . . . . . . . . . . . . . . 80

5.10 Overall timing resolution. . . . . . . . . . . . . . . . . . . . 81

5.11 The Light Injection System. . . . . . . . . . . . . . . . . . . . 83

5.12 Distribution of PMT gains. . . . . . . . . . . . . . . . . . . . . . . . . . . . . . . . . . . . .

5.13 Typical P.TT response curve. . . . . . . . . . . . . . . . . . . . . . . . . . . . . . . . . . .

5.14 Relative response of scintillator strips. . . . . . . . . . . . . . . 87

6.1 Tuning the light output. . . . . . . . . . . . . . . . . . . 92

6.2 Data-MC comparison of light attenuation. . . . . . . . . . . . . . 92

6.3 Tuning the timing resolution. . . . . . . . . . . . . . . . . 93

6.4 Data-MC comparison of time walk. . . . . . . . . . . . . . . 93

6.5 Cosmic ray primary spectrum - low energies . . . . . . . . . . . . . . . 96

6.6 Cosmic ray primary spectrum - high energies . . . . . . . . . . . . . . . 97

6.7 Distribution of muon track planes. . . . . . . . . . . . . . . . . 100

6.8 Distribution of muon zenith angle. . . . . . . . . . . . . . . . . . 101

6.9 Distribution of muon azimuthal angle. . . . . . . . . . . . . . . . 101

6.10 Atmospheric neutrino flux model. . . . . . . . . . . . . . . . . 105

6.11 Cross-sction for $\nu_{\mu}$ CC interactions. . . . . . . . . . . . . . . . . . . . . . . . . . . . . . . . . .

6.12 Cross-section for $\bar{\nu}_{\mu}$ CC interactions. . . . . . . . . . . . . 107

7.1 De-multiplexing atmospheric neutrino events. . . . . . . . . . . . . 115

7.2 Atmospheric neutrino event topologies. . . . . . . . . . . . . . . . . 116

7.3 Track and shower reconstruction. . . . . . . . . . . . . . . . 118

7.4 Reconstructing atmospheric neutrino events. . . . . . . . . . . . . . . . 121

7.5 Reconstruction efficiency for muon tracks (1) . . . . . . . . . . . . . . 123

7.6 Reconstruction efficiency for muon tracks (2) . . . . . . . . . . . . . . . . . . . . . . . . . . . . . .

7.7 Event-by-event track efficiency. . . . . . . . . . . . . . . . . . 124

7.8 Event-by-event track purity . . . . . . . . . . . . . . . . . . 124

7.9 Reconstruction efficiency for vertex showers. . . . . . . . . . . . . . . 125

7.10 Reconstruction of track direction. . . . . . . . . . . . . . . . . . . . . . . . . . . . . . . . . . .

7.11 Distribution of $r m s_{d o w n}$ for stopping muons. . . . . . . . . . . . . . . . 129

7.12 Distribution of $r m s_{u p}-r m s_{\text {down }}$ for stopping muons. . . . . . . . . . . . 129

7.13 Direction reconstruction efficiency for stopping muons (1). . . . . . . . . 130

7.14 Direction reconstruction efficiency for stopping muons (2). . . . . . . . . 130 
7.15 Distribution of $r m s$ for $\nu_{\mu} / \bar{\nu}_{\mu}$ CC events. . . . . . . . . . . . . . . . 131

7.16 Distribution of $r m s_{u p}-r m s_{d o w n}$ for $\nu_{\mu} / \bar{\nu}_{\mu}$ CC events. . . . . . . . . 132

7.17 Direction reconstruction efficiency for $\nu_{\mu} / \bar{\nu}_{\mu}$ CC events. . . . . . . . . . . 132

7.18 Distribution of $\langle Q / p\rangle / \sigma_{\langle Q / p\rangle}$ for stopping muons. . . . . . . . . . . 135

7.19 Charge reconstruction efficiency for stopping muons. . . . . . . . . . . . 135

7.20 Distribution of $\langle Q / p\rangle / \sigma_{\langle Q / p\rangle}$ for $\nu_{\mu} / \bar{\nu}_{\mu}$ CC events. . . . . . . . . . . 136

7.21 Charge reconstruction efficiency for $\nu_{\mu} / \bar{\nu}_{\mu} \mathrm{CC}$ events. . . . . . . . . 136

7.22 Determination of muon momentum from range (1) . . . . . . . . . . . 139

7.23 Determination of muon momentum from range (2) . . . . . . . . . . . . 139

7.24 Determination of muon momentum from curvature (1). . . . . . . . . . . 140

7.25 Determination of muon momentum from curvature (2). . . . . . . . . . 140

7.26 Determination of vertex shower energy from pulse height $(1) \ldots$. . . . . . 141

7.27 Determination of vertex shower energy from pulse height $(2)$. . . . . . . 141

7.28 Angular resolution for $\nu_{\mu} / \bar{\nu}_{\mu}$ CC events (1) . . . . . . . . . . . . 143

7.29 Angular resolution for $\nu_{\mu} / \bar{\nu}_{\mu}$ CC cvents (2). . . . . . . . . . . . 143

8.1 Atmospheric neutrino event classes. . . . . . . . . . . . . . . . . . 147

8.2 Far Detector live time. . . . . . . . . . . . . . . . . . . . . . . . . . . . 149

8.3 Noisc Filtcr. . . . . . . . . . . . . . . . . . . 152

8.4 Containment filter: event classification. . . . . . . . . . . . . . 154

8.5 Containment filter: pulse height in edge regions. . . . . . . . . . . . . . . 155

8.6 Containment filter: pulse height in fiducial region. . . . . . . . . . . . . 155

8.7 Fiducial containment of tracks. . . . . . . . . . . . . . . . . . . . . . . . . . . . . . . . . .

8.8 Containment cuts: (1) Trace. . . . . . . . . . . . . . . . 159

8.9 Containment cuts: (1) Trace cut. . . . . . . . . . . . . . . . . 159

8.10 Containment cuts: (2) Typical background topologies. . . . . . . . . . . 160

8.11 Containment cuts: (2) Topology cuts for FC events. . . . . . . . . . . . . 161

8.12 Containment cuts: (2) Topology cuts for PCDN events . . . . . . . . . . 162

8.13 Containment cuts: (3) Vertex charge. . . . . . . . . . . . . . . 163

8.14 Containment cuts: (3) Vertex charge cut for FC events. . . . . . . . . . . 164

8.15 Containment cuts: (3) Vertex charge cut for PCDN events. . . . . . . . . 164

8.16 Veto shield time window . . . . . . . . . . . . . . 166

8.17 Veto shicld efficiency vs time. . . . . . . . . . . . . . . . . 168

8.18 Data-MC comparison of vetoed cvents (1) . . . . . . . . . . . . . 170

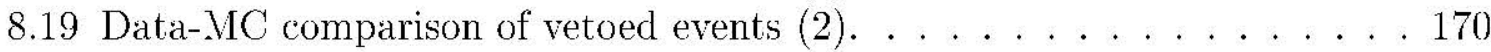

8.20 Timing cuts: (1) Distribution of $r^{m} s_{u p}$ vs rms down . . . . . . . . . . . 175

8.21 Timing cuts: (2) rms cuts. . . . . . . . . . . . . . . . . . . . . . . . . . . . . . . . . . . . . . .

8.22 Timing cuts: (3) rms/range cut. . . . . . . . . . . . . . . . . . . . . . . . . . . . . . . . . . . . .

8.23 Timing cuts: (4) $1 / \beta$ cut. . . . . . . . . . . . . . . . . . 177

8.24 Event selection efficiencies vs neutrino energy. . . . . . . . . . . . . . . . . . . . . . . . . . . . . .

8.25 Event solection officiencies vs muon direction. . . . . . . . . . . . . . . 181

8.26 Other backgrounds - oscillated $\nu_{\mu} / \bar{\nu}_{\mu}$ CC events. . . . . . . . . . . 183

8.27 Candidate atmospheric $\nu_{\mu} / \bar{\nu}_{\mu} \mathrm{CC}$ events (1) FC event. . . . . . . . . . . 187

8.28 Candidate atmospheric $\nu_{\mu} / \bar{\nu}_{\mu}$ CC events (2) PCDN event. . . . . . . 188 
8.29 Candidate atmospheric $\nu_{\mu} / \bar{\nu}_{\mu}$ CC events (3) PCUP event. . . . . . . . 188

8.30 Sclected Events: (1) X-Y interaction points. . . . . . . . . . . . . 189

8.31 Selected Events: (2) Z interaction points. . . . . . . . . . . . . . 190

8.32 Selected Events: (3) Event rates. . . . . . . . . . . . . . 190

9.1 Selection of high resolution sample: (1) track planes. . . . . . . . . . . 194

9.2 Selection of high resolution sample: (2) track range. . . . . . . . . . . . 194

9.3 Selection of high resolution sample: (3) muon direction cut. . . . . . . . . 195

9.4 Selection of high resolution sample: (4) muon charge cut. . . . . . . . . . 195

9.5 Distribution of muon zenith angle: (1) combined $\nu_{\mu} / \bar{\nu}_{\mu}$ events. . . . . . 197

9.6 Distribution of muon zenith angle: (1) separated $\nu_{\mu} / \bar{\nu}_{\mu}$ events. . . . . . . 197

9.7 Atmospheric neutrino up/down ratio as function of $\Delta m_{23}^{2}$. . . . . . . . . 201

9.8 Atmospheric neutrino $\bar{\nu}_{\mu} / \nu_{\mu}$ ratio as function of $\Delta \bar{m}_{23}^{2} . \quad \ldots \ldots$. . . . . 201

9.9 Distribution of reconstructed neutrino energy. . . . . . . . . . . . . . . 203

$9.10 \mathrm{~L} / \mathrm{E}$ resolution: (1) using muon to calculate propagation length. . . . . . 204

$9.11 \mathrm{~L} / \mathrm{E}$ resolution: (2) using neutrino to calculate propagation length. . . . 204

9.12 Distribution of reconstructed $L / E$ : (1) combined $\nu_{\mu} / \bar{\nu}_{\mu}$ events . . . . . . 205

9.13 Distribution of reconstructed $L / E$ : (2) separated $\nu_{\mu} / \bar{\nu}_{\mu}$ events . . . . . . 205

9.14 Systematic errors in $L / E$ distribution. . . . . . . . . . . . . . . . . . . . 207

9.15 Combined $\nu_{\mu} / \bar{\nu}_{\mu}$ oscillation fit: best fit $L / E$ distribution. . . . . . . . . . 210

9.16 Combined $\nu_{\mu} / \bar{\nu}_{\mu}$ oscillation fit: confidence limits (1). . . . . . . . . . 211

9.17 Combined $\nu_{\mu} / \bar{\nu}_{\mu}$ oscillation fit: confidence limits (2). . . . . . . . . . . 212

9.18 Separated $\nu_{\mu} / \bar{\nu}_{\mu}$ oscillation fit: best fit $L / E$ distribution. . . . . . . . . . 214

9.19 Separated $\nu_{\mu} / \bar{\nu}_{\mu}$ oscillation fit: confidence limits. . . . . . . . . . . 215

$9.2025 \mathrm{Kt}-\mathrm{Yr}$ projection: combined $\nu_{\mu} / \bar{\nu}_{\mu}$ oscillation fit. . . . . . . . . . . . 217

$9.2125 \mathrm{Kt}-\mathrm{Yr}$ projection: separated $\nu_{\mu} / \bar{\nu}_{\mu}$ oscillation fit (1). . . . . . . . 218

$9.2225 \mathrm{Kt}$-Yr projection: separated $\nu_{\mu} / \bar{\nu}_{\mu}$ oscillation fit (2) . . . . . . . . 219 


\section{List of Tables}

6.1 MC samples generated for atmospheric neutrino analysis. . . . . . . . 109

8.1 Shield efficiencies (1) rate of cosmic muon tagging. . . . . . . . . . . 167

8.2 Shicld efficicncics (2) rate of accidental signal tagging. . . . . . . . . . 167

8.3 FC/PCDN cvent solection. . . . . . . . . . . . . . . . 172

8.4 PCUP cvent sclection. . . . . . . . . . . . . . . . . . . 178

8.5 Average energy of selected neutrinos. . . . . . . . . . . . . . . 179

8.6 Systematic uncertainties in event selection. . . . . . . . . . . . . . 185

8.7 Atmospheric Neutrino Event Selection Summary. . . . . . . . . . . . . 186

9.1 Event selection: (1) comparison with expectation for oscillations. . . . . . 192

9.2 Event selection: (2) separation of up-going/down-going events. . . . . . . 196

9.3 Event selection: (3) separation of $\nu_{\mu} / \bar{\nu}_{\mu}$ events. . . . . . . . . . . . . . 196

9.4 Event selection: (4) separation of up/down events and $\nu_{\mu} / \bar{\nu}_{\mu}$ events. . . . 196

9.5 Systematic errors in up/down ratio. . . . . . . . . . . . . . . . . . . . . 200

9.6 Systcmatic crrors in $\bar{\nu}_{\mu} / \nu_{\mu}$ ratio. . . . . . . . . . . . . . . . . 200

9.7 Systcmatic crrors in ncutrino oscillation fit. . . . . . . . . . . . . 208 


\section{Chapter 1}

\section{Introduction}

Neutrinos are among the most abundant particles in the universe. However, it is difficult to measure their properties since their interactions with matter are so weak that they arc able to pass through scveral light ycars of matcrial without deviation. Physicist Fred Reines appropriately described neutrinos as: "the most tiny quantity of reality ever imagined by a human being". This ghostly nature makes neutrino physics a difficult science. Neutrino experiments require intense sources of particles and massive underground detectors. Physicist John Bahcall once summed up the challenge to experimenters: "this makes looking for a necdle in a haystack scem casy!". But physicists have time and again risen to the challenge and been rewarded by a rich array of results.

The concept of the neutrino was first postulated in 1930 by Wolfgang Pauli as a solution to a problem obscrved in measurements of nuclear beta decay. It was believed that beta decay was a two-body process involving a nuclear transition and the emission of an electron. However, the electron was found to have a continuous spectrum, an obscrvation which appeared to violate the principle of encrgy conservation. Pauli firmly believed that this law should not be broken under any circumstances and he proposed that a third invisible particle might be emitted in beta decay in addition to the electron and daughter nucleus. This particle would have to be neutrally charged, very light and interact only with the weak force. Pauli's idea was taken up by Enrico Fermi who in 1934 published a quantitative theory of beta decay incorporating the new particle, which Fermi affectionately dubbed the neutrino ("little neutral one") [1].

Fermi's theory accurately described many experimental results and provided strong evidence for the existence of the neutrino. However, the prospect for any direct ob- 
servation of neutrinos seemed remote since the cross-section for neutrino interactions with matter was calculated to be so small that a neutrino could easily pass through the whole Earth without deviation. It was not until two decades later that neutrinos were finally detected by Fred Reines and Clyde Cowan, two physicists from the Los Alamos Laboratory. Reines and Cowan attempted to detect the high flux of neutrinos emitted by nuclear reactors. Their research began in 1951 and was initially dubbed "Project Poltergeist". Their carly attempts were hampered by high backgrounds, but in 1956 they constructed an underground detector beside the Savannah River nuclear reactor in South Carolina and finally observed conclusive evidence of neutrino interactions [2]. Their discovery paved the way for a new branch of study in particle physics.

In 1959, physicist Melvin Schwartz realised that an accelerator beam of neutrinos could be produced by first manufacturing a beam of charged pions and then allowing them to decay. This offered a powerful new technique for studying neutrino interactions. During this time a new problem had also arisen in studies of muon decay. It was believed that the muon decayed into an electron, neutrino and anti-neutrino, but it was not understood why no annihilation took place between the neutrino and anti-neutrino. One proposed solution was that neutrinos might occur in two distinct flavours associated with the clectron and the muon, with clectron neutrinos produced in beta decays and muon neutrinos produced in muon decays. In 1962, Leon Lederman, Jack Steinberger and Schwartz performed the first accelerator neutrino experiment at the Brookhaven Laboratory. They showed that the neutrinos produced from pion decays interacted to produce only muons. Here was compelling evidence that neutrinos occur in more than one gencration [3].

By 1974, two generations of quarks and leptons had been discovered. The first generation contained the up $(u)$ and down $(d)$ quarks, the electron $(e)$ and electron neutrino $\left(\nu_{e}\right)$. The sccond gencration appeared to be a heavicr copy of the first, containing the charm $(c)$ and strange $(s)$ quarks, the muon $(\mu)$ and muon neutrino $\left(\nu_{\mu}\right)$. The observed number of generations soon rose to three. In 1975, a team led by Martin Perl at Stanford discovered the tau lepton $(\tau)$ [4], and two years later, a team led by Leon Lederman at the Fermi Laboratory discovered the bottom quark $(b)[5]$. The discovery of a third generation of quarks and leptons implied the existence of a third type of neutrino and in 2000 , the tau neutrino $\left(\nu_{\tau}\right)$ was finally observed by the DONUT experiment at the Fermi Laboratory [6]. In 1990, the LEP experiment at CERN showed that only three neutrino 
generations exist in nature. LEP collided electrons and positrons at the resonance of the $Z^{0}$ boson and measured its decay width. The results were consistent with a three neutrino universe [7].

Neutrinos are known to be extremely light. In the Standard Model of particle physics they are assigned zero mass. Efforts to directly measure the mass of each neutrino flavour have yielded only upper limits [8] [9] [10] [11] [12] [13]. However, in recent years an indirect technique of probing the mass of neutrinos has produced remarkable results. There is now strong evidence to suggest that that neutrinos undergo transformations between flavours, a quantum mechanical phenomenon known as "neutrino oscillations". This implies that neutrinos possess mass and that neutrino flavours are mixed by weak force. Compelling oscillation signals have now been observed in neutrinos emitted by the sun [14], the atmosphere [15] [16] [17], nuclear reactors [18], and particle accelerators [19]. The MINOS experiment will perform precise measurements of neutrino oscillations using an accelerator beam of neutrinos. The MINOS Far Detector is also being used to study atmospheric neutrino oscillations. This detector is unique in being the first massive underground detector to possess a magnetic field, making the separation of $\nu_{\mu}$ and $\bar{\nu}_{\mu}$ charged current atmospheric neutrino interactions possible for the first time.

This thesis presents an analysis of the first atmospheric neutrino data collected by the Far Detector, and represents the first physics results from the MINOS experiment. In chapter 2, the phenomenology of neutrino oscillations is discussed and the current experimental evidence for neutrino oscillations is revicwed. In chapter 3, an overvicw of the MINOS experiment is presented and the atmospheric neutrino analysis is introduced. In chapter 4, a description of the Far Detector is presented. In chapter 5, the Far Detector timing and energy calibration schemes are outlined. In chapter 6, the simulation of the detector and data and of cosmic ray and atmospheric neutrino interactions within the detector is discussed. In chapter 7 , the methods used to reconstruct physics events in the Far Detector are described. In chapter 8, the separation of atmospheric neutrino events from the high background of cosmic muons is outlined. In chapter 9 , the atmospheric ncutrino $u \mathrm{p} /$ down ratio, $\bar{\nu}_{\mu} / \nu_{\mu}$ ratio, and neutrino oscillation parametcrs are determined, and the expected future sensitivity of the atmospheric neutrino analysis is discussed. 


\section{Chapter 2}

\section{Neutrino Oscillations}

\subsection{Overview}

The current understanding of neutrinos and their interactions is cncapsulated in the Standard Model of electroweak interactions. This accommodates three generations of neutrinos with zcro mass. Each neutrino flavour is paired with a charged lepton: the electron neutrino $\left(\nu_{e}\right)$ with the electron; the muon neutrino $\left(\nu_{\mu}\right)$ with the muon; and the tau neutrino $\left(\nu_{\tau}\right)$ with the tau lepton. In the Standard Model, interactions of leptons are confined within these doublets such that the number of leptons of each flavour remains a conserved quantity. Neutrinos couple only to the weak force and undergo two types of interaction: the charged current (CC) interaction, in which a neutrino exchanges a $W^{ \pm}$ boson with a lepton or quark to produce its partner charged lepton; and the neutral current $(\mathrm{NC})$ interaction, in which a neutrino exchanges a $Z^{0}$ boson. The large masses of these exchange bosons mean that the cross-sections for neutrino interactions with matter at low energies are extremely small.

There is no known symmetry of nature that requires neutrinos to be massless. In recent years experiments have begun to challenge the Standard Model assumption that neutrinos have no mass. There is now compelling evidence that neutrinos undergo transitions between flavours, a quantum mechanical phenomenon known as "neutrino oscillations". This would imply that neutrinos possess a small but non-zero mass, and that the leptonic flavours are mixed by the weak interaction, breaking the conservation of lepton number for each flavour.

Evidence of neutrino oscillations has been observed in a number of neutrino sources, 
both natural and artificial. The seminal neutrino oscillation experiments measured solar neutrinos produced by thermonuclear reactions at the centre of the sun, and atmospheric neutrinos produced by cosmic ray interactions in the Earth's atmosphere. In both sources, a deficit was observed in the measured neutrino flux relative to the Standard Model expectation. More recently, evidence for oscillations has also been observed in neutrinos manufactured in nuclear reactors and accelerator beams.

\subsection{Theory of Neutrino Oscillations}

If neutrinos possess mass, then there exists a spectrum of neutrino mass eigenstates $\left|\nu_{i}\right\rangle(i=1,2,3)$. The neutrino eigenstates $\left|\nu_{\alpha}\right\rangle(\alpha=e, \mu, \tau)$ that couple to the weak force are in general not identical to these mass eigenstates, but can be expressed as a linear combination of them as follows:

$$
\left|\nu_{\alpha}\right\rangle=\sum_{i=1,2,3} U_{\alpha i}\left|\nu_{i}\right\rangle
$$

where $U$ is a unitary lepton mixing matrix. For neutrino propagation through a vacuum, the neutrino mass eigenstates are also free particle eigenstates, and a state $\left|\nu_{i}\right\rangle$ at position $\mathbf{x}$ with four-momentum $\mathbf{p}_{i}$ cvolves as follows:

$$
\left|\nu_{i}(\mathbf{x})\right\rangle=e^{-i \mathbf{p}_{i} \cdot \mathbf{x}}\left|\nu_{i}\right\rangle
$$

Therefore a neutrino produced in a flavour state $\left|\nu_{\alpha}\right\rangle$ evolves as follows:

$$
\left|\nu_{\alpha}(\mathbf{x})\right\rangle=\sum_{i=1,2,3} e^{-i \mathbf{p}_{i} \cdot \mathbf{x}} U_{\alpha i}\left|\nu_{i}\right\rangle
$$

Assuming that the mass states are produced with a common energy and that the neutrino masses are small compared to this encrgy, the phase $\mathbf{p}_{i} \cdot \mathbf{x}$ of each state can be approximated as: $\mathbf{p}_{i} \cdot \mathbf{x}=E t-p_{i} x \approx\left(m_{i}^{2} / 2 E\right) L$. If the neutrino masses $m_{1}, m_{2}, m_{3}$ are different, then the phase of each mass state will evolve at a different rate and a neutrino produced in an initially pure flavour state will develop contributions from the other flavours over time. Therefore a neutrino produced as one flavour may be detected as a different flavour at a later time. The probability that a flavour state $\nu_{\alpha}$ transforms into a flavour state $\nu_{\beta}$ after a distance $L$ is given by: 


$$
P\left(\nu_{\alpha} \rightarrow \nu_{\beta}\right)=\left|\left\langle\nu_{\beta} \mid \nu_{\alpha}(L)\right\rangle\right|^{2}
$$

Substituting from Equations 2.1 and 2.3 gives the following transition probabilities:

$$
\begin{aligned}
P\left(\nu_{\alpha} \rightarrow \nu_{\beta}\right) & =\delta_{\alpha \beta}-4 \sum_{i<j} \operatorname{Re}\left(U_{\alpha i}^{*} U_{\beta i} U_{\alpha j} U_{\beta j}^{*}\right) \sin ^{2} \frac{\Delta m_{i j}^{2} L}{4 E} \\
& +2 \sum_{i<j} \operatorname{Im}\left(U_{\alpha i}^{*} U_{\beta i} U_{\alpha j} U_{\beta j}^{*}\right) \sin \frac{\Delta m_{i j}^{2} L}{2 E}
\end{aligned}
$$

where $\Delta m_{i j}^{2}$ is the squared mass splitting between the $i^{\text {th }}$ and $j^{\text {th }}$ mass states. Restoring units of $\hbar$ and $c$ in Equation 2.5 requires the inclusion of an additional numerical factor as follows:

$$
\frac{\Delta m_{i j}^{2}}{4 E} L \cong 1.27 \Delta m_{i j}^{2}\left(\mathrm{eV}^{2}\right) \frac{L(\mathrm{~km})}{E(\mathrm{GcV})}
$$

The transition probability in Equation 2.5 is periodic with the distance of neutrino propagation. Therefore, if the neutrino mass states mix and possess different masses then neutrinos will oscillate between flavours during flight.

The transition probabilities for anti-neutrino flavours can be obtained from the transition probabilities for neutrinos. Assuming CPT invariance holds:

$$
P\left(\bar{\nu}_{\alpha} \rightarrow \bar{\nu}_{\beta}\right)=P\left(\nu_{\beta} \rightarrow \nu_{\alpha}\right)
$$

Setting $\nu_{\alpha} \leftrightarrow \nu_{\beta}$ in Equation 2.5, this becomes:

$$
P\left(\bar{\nu}_{\alpha} \rightarrow \bar{\nu}_{\beta} ; U\right)=P\left(\nu_{\alpha} \rightarrow \nu_{\beta} ; U^{*}\right)
$$

Therefore, the anti-neutrino transition probabilities are obtained from the neutrino transition probabilities by replacing the mixing matrix $U$ by its complex conjugate $U^{*}$. If the mixing matrix is complex, then different probabilities are obtained for neutrinos and anti-neutrinos in cases where neutrinos undergo transitions between different flavours. This is because in these cases, the last term in Equation 2.5 carries an opposite sign for neutrinos and anti-neutrinos. This represents a violation of $C P$ invariance.

The idea of mixing of massive neutrinos was first introduced in 1962 by Maki, Nak- 
agawa and Sakata [20], and the phenomenology of neutrino oscillations was first discussed in 1967 by Pontecorvo [21]. Hence the unitary matrix $U$ is commonly known as the Pontecorvo-Maki-Nakagawa-Sakata (PMNS) matrix. The mixing of three neutrino mass states can be described using threc mixing angles and threc complex phases. A common parameterization of the PMNS mixing matrix is given by the following product of matrices:

$$
U=\left(\begin{array}{ccc}
1 & 0 & 0 \\
0 & c_{23} & s_{23} \\
0 & -s_{23} & c_{23}
\end{array}\right)\left(\begin{array}{ccc}
c_{13} & 0 & s_{13} e^{-i \delta} \\
0 & 1 & 0 \\
-s_{13} e^{i \delta} & 0 & c_{13}
\end{array}\right)\left(\begin{array}{ccc}
c_{12} & s_{12} & 0 \\
-s_{12} & c_{12} & 0 \\
0 & 0 & 1
\end{array}\right)\left(\begin{array}{ccc}
e^{i \alpha} & 0 & 0 \\
0 & e^{i \beta} & 0 \\
0 & 0 & 1
\end{array}\right)
$$

Here $c_{i j}=\cos \theta_{i j}$ and $s_{i j}=\sin \theta_{i j}$ where $\theta_{i j}$ is the mixing angle between the $i^{\text {th }}$ and $j^{\text {th }}$ states. The complex phase $\delta$ is known as the Dirac phase and is the leptonic analogue of the single complex phase in the quark sector. The size of the Dirac phase determines the extent of CP violation in the neutrino sector. The complex phases $\alpha$ and $\beta$ are known as Majorana phases and have physical consequences if neutrinos are Majorana particles, identical to their anti-particles. The Majorana phases do not affect the neutrino oscillation probabilities.

Neutrino oscillations can be detected experimentally by measuring the change in the flavour composition of neutrino sources over long distances. The experimental signature for neutrino oscillations is a flavour composition that differs from the Standard Model expectation periodically as a function $L / E$. The mixing angles and mass splittings can be determined experimentally by measuring the period of the neutrino oscillations. The mass splittings are determined by measuring the period of the oscillations; and the mixing angles are determined by measuring the amplitude of the oscillations.

The three-flavour neutrino oscillations described by Equation 2.5 can be greatly simplified in cases of practical importance. The current empirical evidence suggests that the neutrino mass hierarchy and mixing matrix follow a regime in which the mass splitting $\left|\Delta m_{23}^{2}\right|$ is much greater than the mass splitting $\left|\Delta m_{12}^{2}\right|$; the two mixing angles $\theta_{12}$ and $\theta_{23}$ are large; and the third mixing angle $\theta_{13}$ is small. In this regime, the three-flavour oscillations of Equation 2.5 effectively decouple into the following sets of two-flavour oscillations: 
- Long Range Oscillations: Over long ranges where $\Delta m_{12}^{2} L / E \sim O(1)$, the dominant mode of oscillations is $\nu_{e} \leftrightarrow \nu_{\mu} / \nu_{\tau}$, controlled by the squared mass splitting $\Delta m_{12}^{2}$ and mixing angle $\theta_{12}$. This mode of oscillations is associated with measurements of $\nu_{e}$ disappearance in solar neutrinos and long bascline reactor experiments. The oscillation probability is given by:

$$
P\left(\nu_{e} \leftrightarrow \nu_{\mu} / \nu_{\tau}\right) \simeq \sin ^{2} 2 \theta_{12} \sin ^{2} \frac{1.27 \Delta m_{12}^{2} L}{E}
$$

- Short Range Oscillations : Over short ranges where $\Delta m_{23}^{2} L / E \sim O(1)$, the dominant mode of oscillations is $\nu_{\mu} \leftrightarrow \nu_{\tau}$, controlled by the squared mass splitting $\Delta m_{23}^{2}$ and mixing angle $\theta_{23}$. This short range mode of oscillations is associated with measurements of $\nu_{\mu}$ disappcarance in atmospheric neutrinos and long bascline neutrino experiments. The oscillation probability is given by:

$$
P\left(\nu_{\mu} \leftrightarrow \nu_{\tau}\right) \simeq \sin ^{2} 2 \theta_{23} \sin ^{2} \frac{1.27 \Delta m_{23}^{2} L}{E}
$$

- Sub-Dominant Short Range Oscillations : Over short ranges, a subdominant oscillation mode $\nu_{e} \leftrightarrow \nu_{\mu} / \nu_{\tau}$ is permitted at a level controlled by the small mixing angle $\theta_{13}$. This mode of oscillations is associated with searches for $\nu_{e}$ disappearance in short baseline reactor neutrino experiments. The oscillation probability is given by:

$$
P\left(\nu_{e} \leftrightarrow \nu_{\mu} / \nu_{\tau}\right) \simeq \sin ^{2} 2 \theta_{13} \sin ^{2} \frac{1.27 \Delta m_{23}^{2} L}{E}
$$

Neutrino oscillation experiments have been constructed with a range of characteristic $L$ and $E$ to study each of these oscillation modes and probe the different regions of oscillation parameter space. For an oscillation experiment to be sensitive to a particular mass splitting $\Delta m^{2}$, the characteristic $L / E$ of the incident neutrino flux must satisfy $L / E \simeq \pi / \Delta m^{2}$. This ensures that the neutrino flux is sampled in the $L / E$ region that maximizes the oscillation probability. For cases in which $L / E \ll \pi / \Delta m^{2}$, the oscillation probability is small and consequently flavour transitions do not take place at a detectable level. For cases in which $L / E \gg \pi / \Delta m^{2}$, the oscillation probability averages to $\frac{1}{2} \sin ^{2} \theta$ and the size of the mass splitting cannot be resolved. 


\subsection{Neutrino Oscillation Experiments}

The detection of neutrinos is based on the observation of particles produced in charged current or neutral current neutrino interactions. The tiny neutrino cross-section means that intense neutrino sources and large detectors are required to obtain reasonable event rates. Neutrino detectors must also be placed underground to provide shiclding against cosmic radiation incident on the Earth's surface.

A range of neutrino experiments have becn constructed to study neutrino oscillations. These experiments fall into two categories: "disappearance" experiments search for a deficit in the dominant flavour component of a neutrino source relative to the initial flux; and "appearance" experiments scarch for an excess in a sub-dominant flavour component of a neutrino source relative to the initial flux. Both types of experiment require an accurate measurement or simulation of the initial flux of neutrinos in order to infer that neutrino flavour transformations have taken place. Both natural and artificial sources of neutrinos are used to study oscillations. The current experimental status is summarized below.

\subsubsection{Solar Neutrinos}

The sun is powered by a chain of nuclear fusion reactions taking place in the solar core. These reactions generate energy and also produce an intense flux of $\nu_{c}$. Solar neutrinos represent the largest known flux of neutrinos incident on the Earth's surface. The dominant chain of fusion reactions is the "pp chain", in which four hydrogen nuclei fuse together to form a helium nucleus. The combined effect of these reactions is:

$$
4 p+2 e^{-} \rightarrow{ }^{4} \mathrm{He}+2 \nu_{e}+27 \mathrm{MeV}
$$

The structure and dynamics of the solar core are simulated by the Standard Solar Model (SSM) [22]. The SSM is used to determine the rate of cach fusion reaction and calculate the emitted flux of solar neutrinos. Figure 2.1 shows the reactions that form the $p p$ chain, and the energy spectrum of solar neutrinos predicted by the SSM. The majority of solar neutrinos $(>90 \%)$ are emitted by the primary $p p$ interaction $p+p \rightarrow d+e^{+}+\nu_{e}$ that initiates the $p p$ chain. However, the energies of these neutrinos are low $(<0.42 \mathrm{MeV})$. At higher energies, the decay ${ }^{8} B \rightarrow{ }^{8} B e^{*}+e^{+}+\nu_{e}$ produces neutrinos with energies up to $14.1 \mathrm{MeV}$, and the interactions ${ }^{7} \mathrm{Be}+e^{-} \rightarrow^{7} \mathrm{Li}+\nu_{e}$ and 

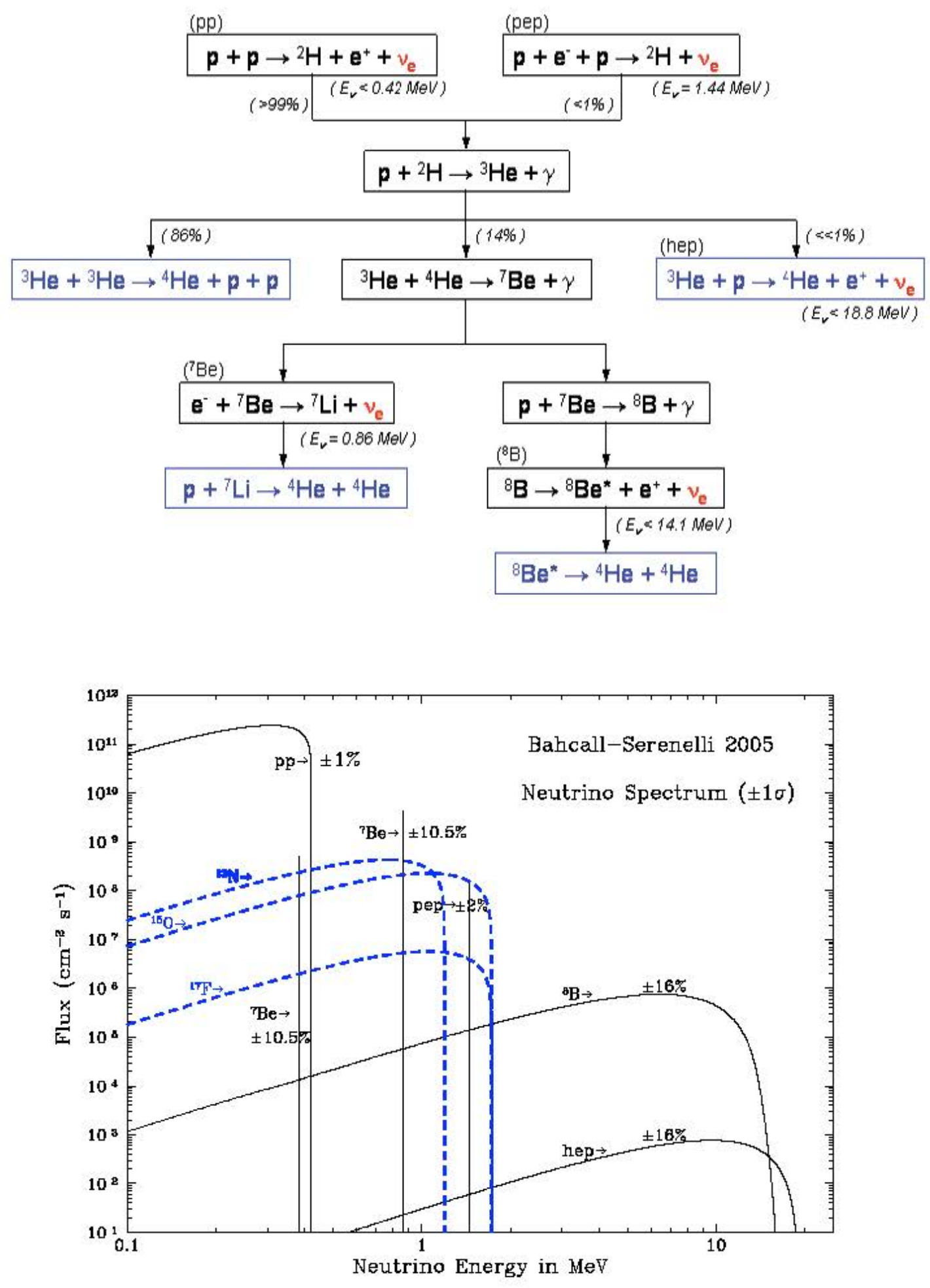

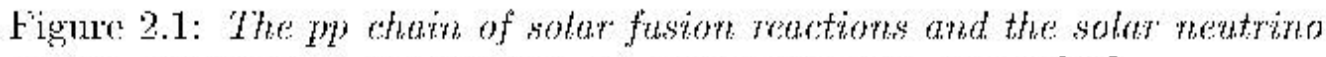

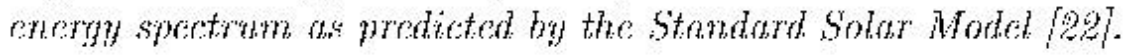


$p+e^{-}+p \rightarrow d+\nu_{e}$ produce mono-energetic peaks at $0.86 \mathrm{MeV}$ and $1.44 \mathrm{MeV}$.

The flux of solar neutrinos incident on the Earth's surface can be measured experimentally using large underground detectors. The pioneering solar neutrino experiment was the Homestake radiochemical detector located in Lead, South Dakota [23]. Homestake used 615 tons of $\mathrm{C}_{2} \mathrm{Cl}_{4}$ to detect solar neutrinos by the inverse beta decay process: $\nu_{e}+{ }^{37} \mathrm{Cl} \rightarrow{ }^{37} \mathrm{Ar}+e^{-}$. This process has a threshold energy of $0.81 \mathrm{MeV}$, making Homestake sensitive chiefly to ${ }^{8} B$ neutrinos. The tiny neutrino cross-section restricted the interaction rate in the Homestake detector to approximately 1 event per day. The ${ }^{37} A r$ was allowed to accumulate over several months and was then chemically extracted from the tank. ${ }^{37} \mathrm{Ar}$ is radioactive with a half life of 35 days, so the ${ }^{37} \mathrm{Ar}$ yicld was measured using a low background counter. The typical ${ }^{37} A r$ yield obtained from each run was typically 15 atoms. However, Homestake was capable of extracting these atoms with $\geq 90 \%$ efficiency. The Homestake experiment ran from 1968-1995 and consistently measured a $\nu_{e}$ rate lower than that predicted by the SSM. The average solar neutrino flux was calculated to be $2.56 \pm 0.16 \pm 0.16 \mathrm{SNL}$ ( 1 SNU $\equiv 1$ interaction per $10^{36}$ atoms per second) compared with an expectation of $7.6_{-1.1}^{+1.3}$ SNU [24]. The observed $\nu_{e}$ deficit became known as the "solar neutrino problem".

A new generation of radiochemical experiments was developed in the 1980s and 1990s by SAGE [25] and GALLEX [26] using gallium to detect solar neutrinos by the inverse beta decay process: $\nu_{e}+{ }^{71} G a \rightarrow^{71} G e+e^{-}$. This process has a threshold energy of $0.23 \mathrm{McV}$ cnabling SAGE and GALLEX to perform the first measurements of solar neutrinos emitted by the primary $p p$ interaction. Like Homestake, both SAGE and GALLEX observed a deficit in the rate of $\nu_{e}$ relative to the SSM prediction, measuring a $\nu_{e}$ flux of $70.8_{-5.2}^{+5.3}{ }_{-3.2}^{+3.7} \mathrm{SNL}[27]$ and $77.5_{-6.2}^{+6.2}{ }_{-1.7}^{+1.3}$ SNU [28] respectively compared with an expectation of $128_{-7}^{+9}$ SNU [29] for both experiments.

A different technique of solar neutrino detection was employed by the Kamiokande experiment [30], located beneath . It. Ikenoyama in Japan. Kamiokande used a detector containing $3 \mathrm{kT}$ of ultra-pure water and instrumented by 900 P.TTs to detect newtrino interactions by the clastic scattering process: $\nu_{x}+e^{-} \rightarrow e^{-}+\nu_{x}$. All threc neutrino flavours contribute to this process through $\mathrm{NC}$ scattering, but a large additional contribution arises from $\nu_{e}$ CC scattering. This makes the cross-section for $\nu_{e}$ approximately six times larger than the cross-section for $\nu_{\mu}$ and $\nu_{\tau}$. Neutrino interactions were detected by observing the Cerrenkov light emitted by recoil electrons. The 
close correlation between the direction of recoil and the direction of the incident neutrino assisted the separation of the solar neutrino signal from the background natural radioactivity. Solar neutrinos could be positively identified above an energy threshold of $7 \mathrm{McV}$ making Kamiokande sensitive chicfly to ${ }^{8} B$ neutrinos. Kamiokande collected solar neutrino data from 1987-1995. Like the radiochemical experiments, it observed a significant deficit in the rate of solar neutrinos, measuring a neutrino flux corresponding to $49.6_{-4.2}^{+1.1}{ }_{-4.8}^{+1.8} \%$ of the SSM expectation [31].

A large scale successor to Kamiokande, the Super-Kamiokande (SKK) experiment (see Figure 2.2) [32], has been collecting data since 1996. SKK uses $50 \mathrm{kT}$ of ultra-pure water to detect neutrino interactions by measuring the Cerrenkov radiation emitted by charged particles produced in the interactions. The large mass of the SKK detector provides a high neutrino interaction rate enabling flux measurements to be performed with high precision. The SKK detector is divided into two parts. An inner detector containing $20 \mathrm{kT}$ of water and instrumented with 11,000 PMTs is used as a fiducial volume for detection of neutrino events. An outer detector containing $30 \mathrm{kT}$ and instrumented with 2,000 PMTs is used to veto through-going cosmic muons and low energy backgrounds. Significant improvements in water purity levels have been achieved over Kamiokande, reducing backgrounds from natural radioactivity and cnabling SKK to detect solar neutrinos down to $5 \mathrm{MeV}$. SKK has performed a precise determination of the solar neutrino flux by measuring neutrino-electron scattering [33]. Figure 2.3 shows the angular distribution of recoil clectrons relative to the position of the sun. This shows a clear forward peak from solar neutrino interactions above a flat background distribution. A measurement of the solar neutrino flux is obtained by fitting the angular distribution. SKK observes a significant deficit in the $\nu_{e}$ rate, measuring a neutrino flux corresponding to $46 . \bar{z} \pm 0 . \bar{j} \pm 1.3 \%$ of the SSM expectation [34].

Neutrino oscillations offer a resolution to the solar neutrino problem since transitions between $\nu_{e}$ and $\nu_{\mu} / \nu_{\tau}$ in solar neutrinos result in a deficit in the measured $\nu_{e}$ flux relative to the SSM expectation. All of the experiments described above are sensitive primarily to the $\nu_{e}$ flux and so observe the disappearance of $\nu_{e}$ but not the corresponding appearance of $\nu_{\mu}$ and $\nu_{\tau}$. Recently, compelling evidence in favour of neutrino oscillations in has been observed in solar neutrinos by the Sudbury Neutrino Observatory (SNO) using a detector uniquely sensitive to all three neutrino flavours. 


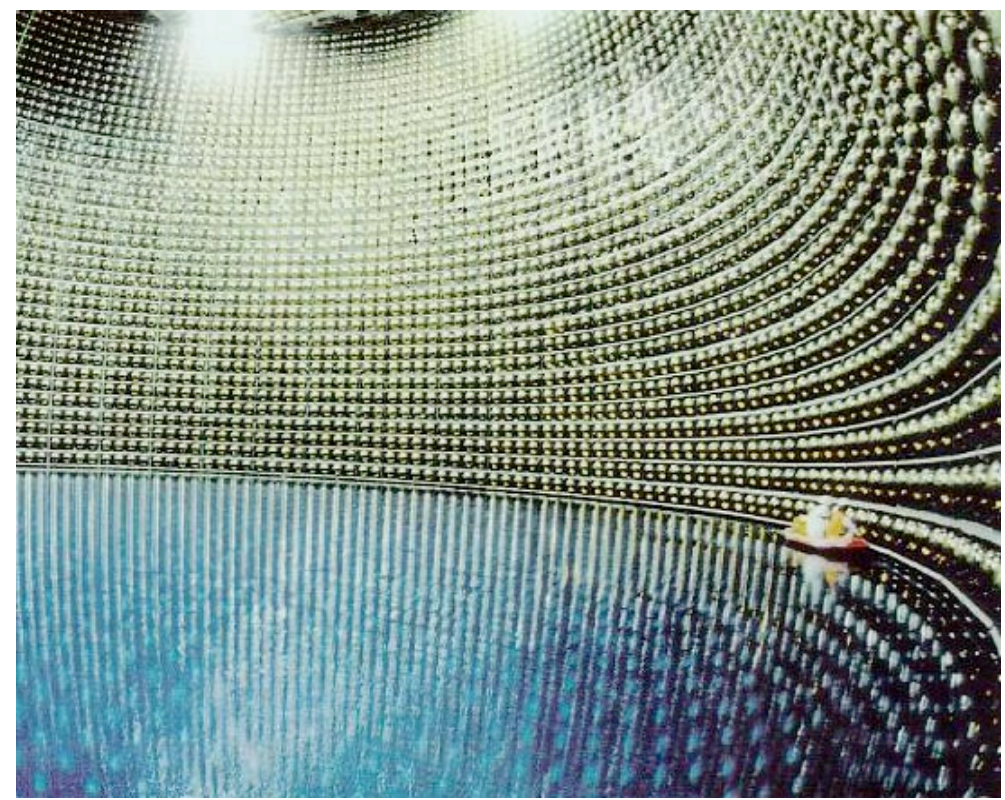

Figure 2.2: Super-Kamiokande uses a $50 \mathrm{kT}$ water Cĕrenkov detector to measure the neutrino interactions. Its large mass delivers a high interaction rate, enabling flux measurements to be performed with high precision.

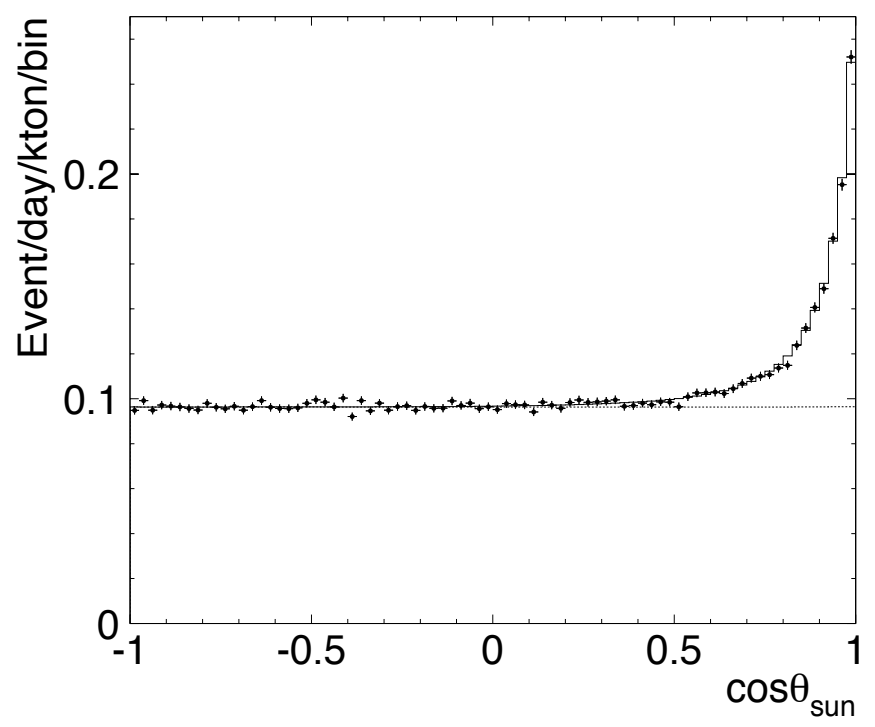

Figure 2.3: The angular distribution of neutrino-electron scattering measured by Super-Kamiokande [33]. The peak at +1 is due to solar neutrinos. 


\subsubsection{SNO}

SNO is a $1 \mathrm{kT}$ heavy water $\left(\mathrm{D}_{2} \mathrm{O}\right)$ Cěrenkov detector (see Figure 2.4) located in Sudbury, Canada [35]. The use of $\mathrm{D}_{2} \mathrm{O}$ to detect solar neutrinos provides a unique sensitivity to all three neutrino flavours. Three modes of neutrino detection are possible through three different interactions: charged current (CC) interactions $\left(\nu_{e}+d \rightarrow p+p+e^{-}\right)$, sensitive only to $\nu_{e}$; neutral current ( $\mathrm{NC}$ ) interactions $\left(\nu_{x}+d \rightarrow n+p+e^{-}\right)$, sensitive to all three flavours of neutrino in equal amounts; and elastic scattering (ES) interactions $\left(\nu_{x}+e^{-} \rightarrow e^{-}+\nu_{e}\right)$, sensitive to all threc flavours of neutrino but with reduced crosssections for $\nu_{\mu}$ and $\nu_{\tau}$. The measurements of each interaction type can be combined to obtain separate measurements of the $\nu_{e}$ flux and the $\nu_{\mu} / \nu_{\tau}$ flux. The energy threshold of the SNO analysis is $5 \mathrm{McV}$, making SNO sensitive chicfly to ${ }^{8} B$ solar neutrinos.

The SNO experiment is being performed in three phases, with the $\mathrm{NC}$ sensitivity enhanced for each new phase of the experiment. In the first phase, NC events are detected by observing the emission of a $6 \mathrm{McV}$ gamma ray following the capture of the emitted neutron; in the second phase, 2 tons of salt $(\mathrm{NaCl})$ are added to the water, increasing the neutron capture efficiency and the gamma ray multiplicity; in the third phase, the salt is removed and an array of ${ }^{3} H e$ proportional counters are deployed in the water to directly detect the neutrons. The experiment is currently in its third phase. Figure 2.5 shows the results of the second phase [36]. The non- $\nu_{e}$ component of the flux $\left(\phi_{\mu \tau}\right)$ is plotted against the $\nu_{e}$ component of the flux $\left(\phi_{e}\right)$. The SNO measurement of the $C C, N C$ and $E S$ interactions are plotted along with the SKK measurement of $E S$ interactions and the SSM prediction. All the measurements are consistent with the SSM expectation and with each other, and meet at a common point at which the non- $\nu_{e}$ flux has a non-zero valuc. This provides compelling evidence that a non- $\nu_{e}$ component of the solar neutrino flux exists, and that when combined with the $\nu_{e}$ component, the total flux is in good agreement with the SSM prediction.

The SNO measurements of the solar neutrino flux can be used to determine the oscillation parameters $\Delta m_{12}^{2}$ and $\theta_{12}$ that control the long range $\nu_{e} \leftrightarrow \nu_{\mu} / \nu_{\tau}$ oscillations. The analysis must incorporate the effects on the oscillations of neutrino interactions in mattcr. As neutrinos propagate through a medium, they undergo forward scattering interactions within the medium. All three neutrino flavours undergo NC scattering, but electron neutrinos undergo additional CC scattering off electrons. This means that electron neutrinos experience an additional potential in the medium relative to muon and 


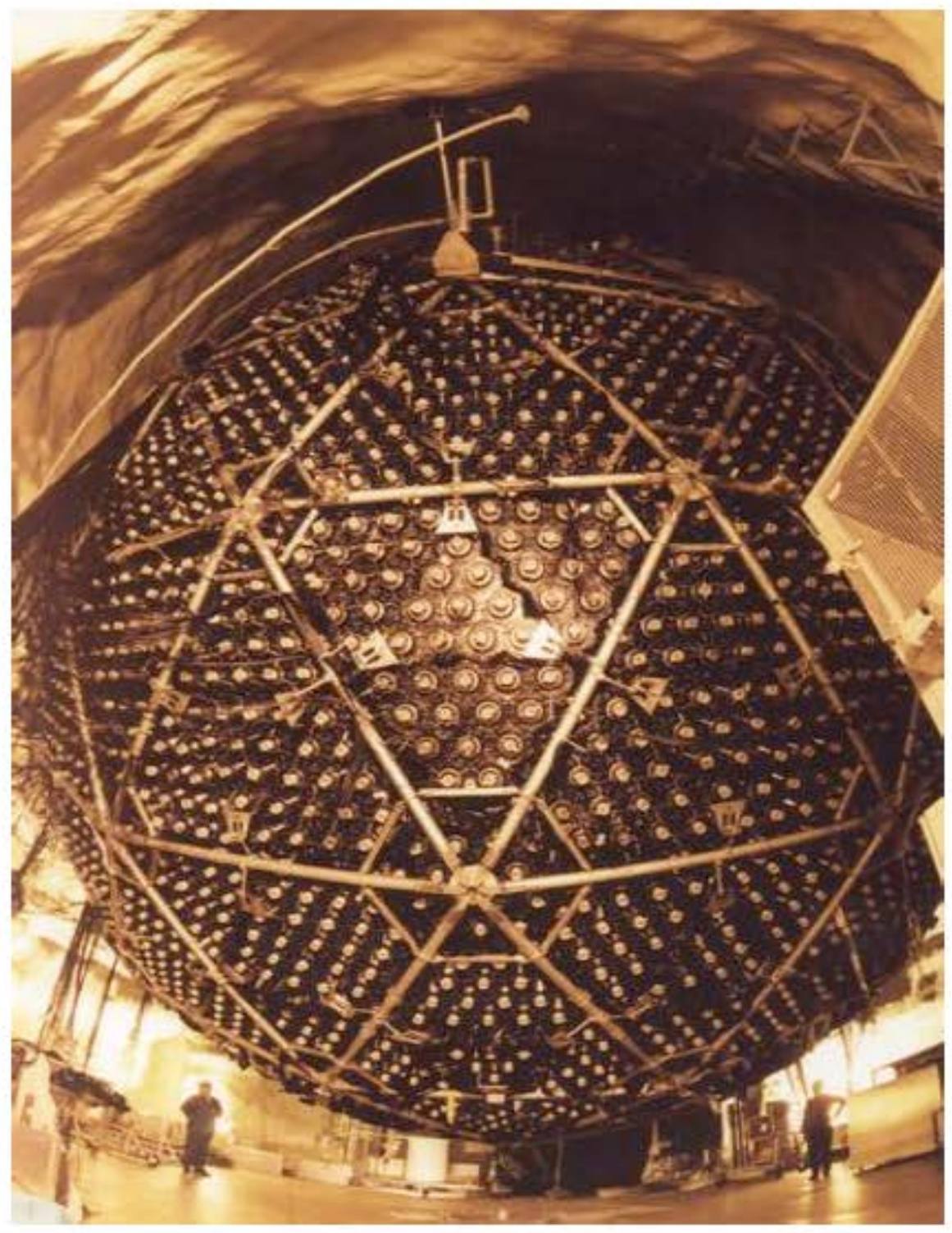

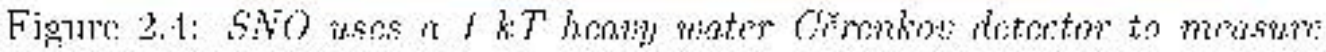

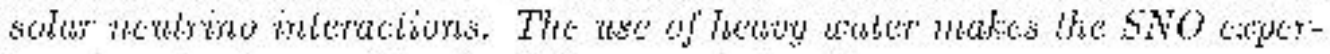

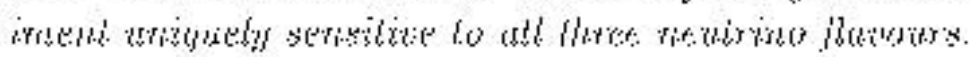




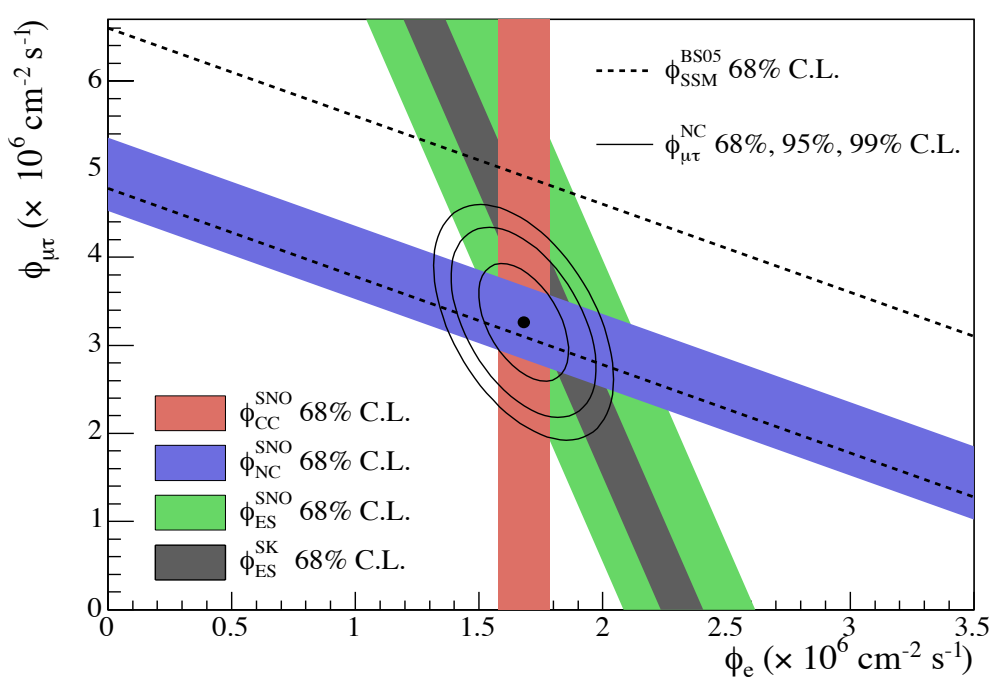

Figure 2.5: The results from the second phase of the SNO experiment [36]. The flux of $\nu_{\mu}+\nu_{\tau}$ is plotted against flux of $\nu_{e}$. The SNO measurements of $C C, N C$ and ES neutrino interactions and the SKK measurement of ES neutrino interactions are indicated by the filled bands, while the flux predicted by the SSM is indicated by the dotted lines. The $\pm 1 \sigma$ uncertainties are represented by the intercepts on the axes.

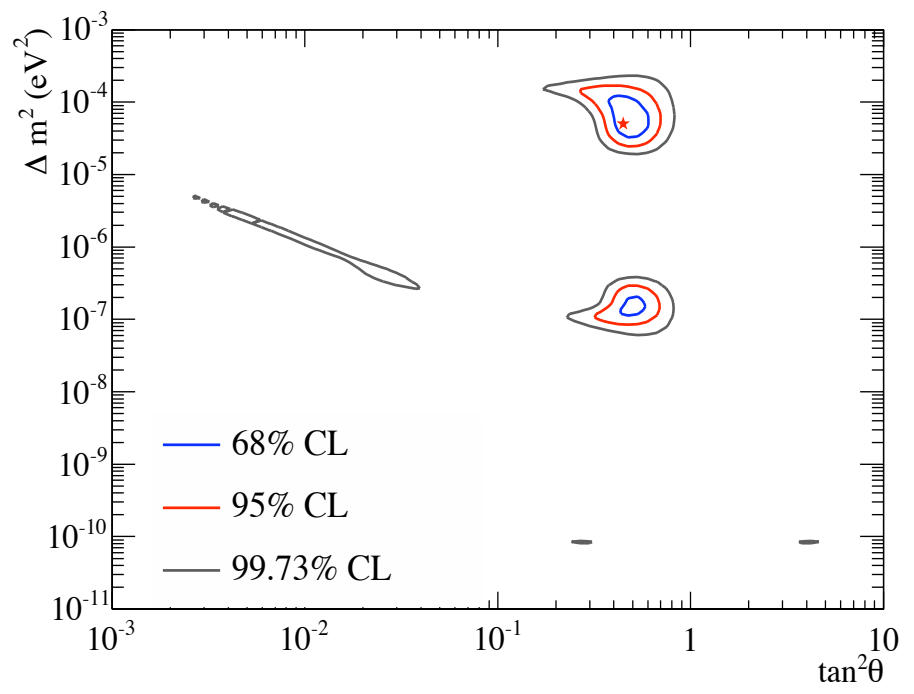

Figure 2.6: The confidence limits for the oscillation parameters $\Delta m_{12}^{2}$ and $\theta_{12}$ obtained from and analysis of the results from the second phase of the SNO experiment [36]. The best fit point is indicated by a star. 
tau neutrinos, modifying the mass states in the medium and altering the mixing angles and mass splittings. This is known as the Mikheyev-Smirnov-Wolfenstein (MSW) effect [37] [38]. The high density of electrons at the centre of the sun heavily distorts the neutrino mass states and therefore significantly affects the transitions between the neutrino flavours.

Figure 2.6 shows the confidence limits for the parameters $\Delta m_{12}^{2}$ and $\theta_{12}$ calculated from an analysis of SNO data assuming two-flavour oscillations. The fit is consistent with more than one region of parameter space, but favours "Large Mixing Angle" (LMA) oscillations. The best fit point is: $\Delta m_{12}^{2}=5.0_{-1.8}^{+6.2} \times 10^{-5} \mathrm{eV}^{2}, \tan ^{2} \theta_{12}=0.45_{-0.10}^{+0.11}$. SNO has also carricd out a global analysis of all solar neutrino expcriments. This strongly favours LMA oscillations, excluding all other regions of parameter space at $99 \%$ confidence. The best fit point is: $\Delta m_{12}^{2}=6.5_{-2.3}^{+4.4} \times 10^{-5} \mathrm{eV}^{2}, \tan ^{2} \theta_{12}=0.45_{-0.08}^{+0.09}$.

\subsubsection{KamLAND}

The oscillation signal observed in solar neutrinos has been confirmed by the KamLAND long bascline reactor neutrino experiment [39]. The KamLAND experiment is measuring the flux of $\bar{\nu}_{e}$ produced by a cluster of nuclear power reactors. Assuming that CPT invariance holds, the KamLAND $\bar{\nu}_{e}$ measurements can be combined with the solar $\nu_{e}$ measurements to constrain the solar neutrino oscillation parameters. KamLAND measures neutrinos produced by 52 reactor cores located at 16 commercial power stations in Japan. The neutrinos are detected using a $1 \mathrm{kT}$ liquid scintillator detector located bencath Mt. Ikcnoyama in Japan. Neutrinos are cmitted by nuclear power reactors as a result of beta decays of neutron rich fission fractions. The typical energies of these neutrinos are $E \simeq 3 \mathrm{McV}$. In order to probe the mass splittings of $\Delta \mathrm{m}^{2} \simeq 10^{-5} \mathrm{cV}^{2}$ obtained from global fits to solar neutrino data, the reactor neutrinos must be detected at propagation distances of order $L \simeq 200 \mathrm{~km}$. The reactors are distributed at distances of $L \simeq 100-700 \mathrm{~km}$ from the KamLAND detector.

KamLAND detects $\bar{\nu}_{e}$ using the inverse beta decay process: $\bar{\nu}_{e}+p \rightarrow n+e^{+}$. The signature of a $\bar{\nu}_{e}$ interaction is prompt scintillation light produced by the $e^{+}$followed by a $2 \mathrm{McV}$ gamma ray produced by the capture of the $n$ on hydrogen. The $\bar{\nu}_{e}$ encrgy is estimated from the measured prompt $e^{+}$scintillation light. The expected $\bar{\nu}_{e}$ in the absence of oscillations is calculated using operational data provided by each nuclear power plant. KamLAND has published an analysis of data acquired between March 


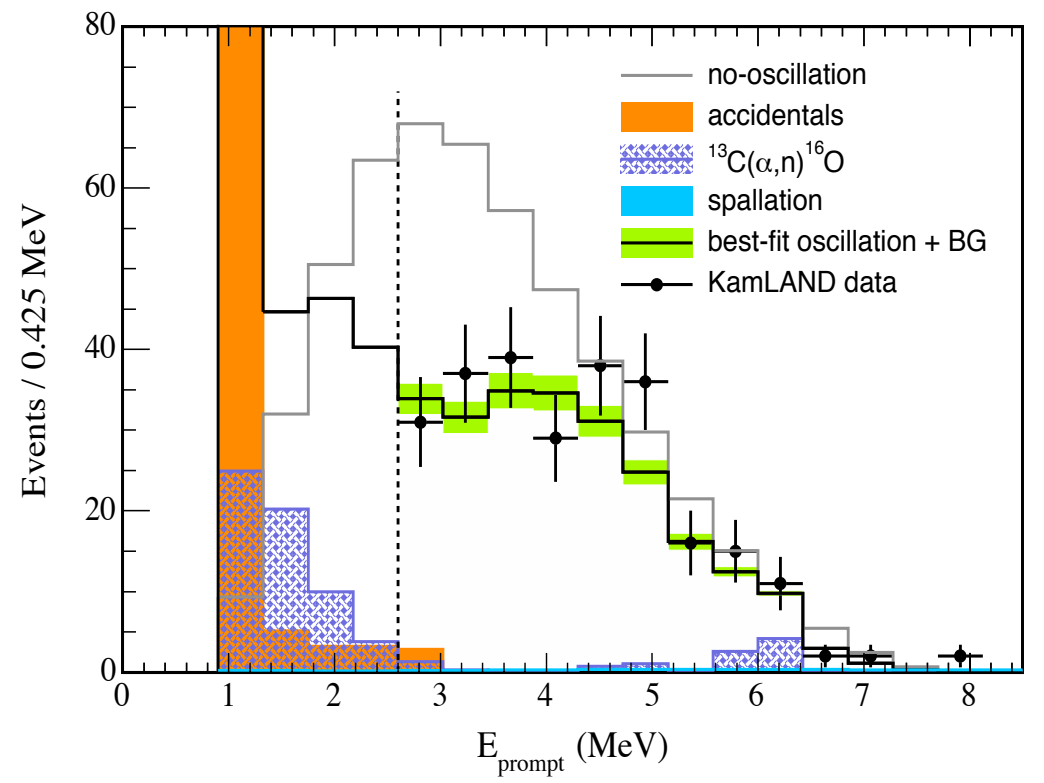

Figure 2.7: Energy spectrum of prompt $e^{+}$from $\bar{\nu}_{e}$ interactions detected by KamLAND [40]. There is a significant deficit in the measured spectrum relative to the no-oscillation expectation.
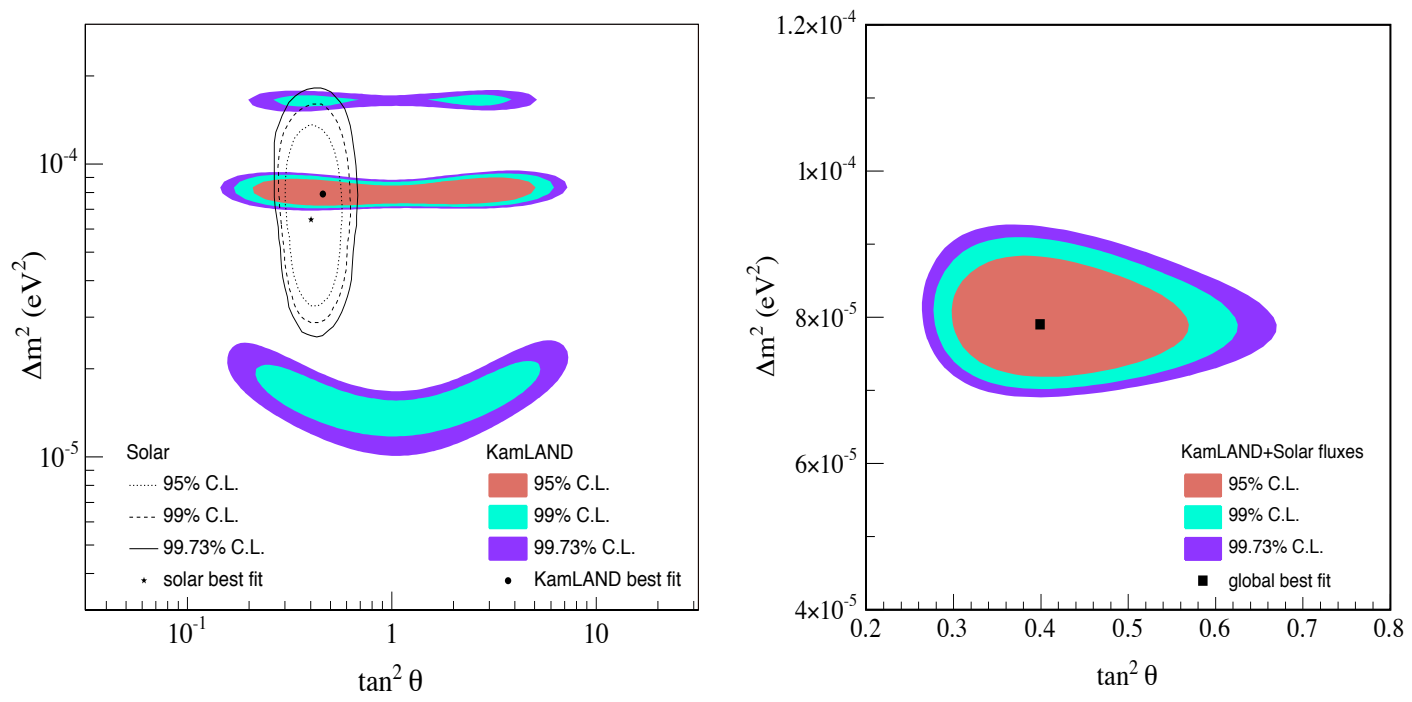

Figure 2.8: (a) Allowed region of oscillation parameter space from analysis of KamLAND data (indicated by shaded regions) and global analysis of solar neutrino data (indicated by lines) (b) Allowed region of oscillation parameter space from combined analysis of KamLAND and solar neutrino data. Figure taken from [40]. 
2002 and January 2004 [40]. KamLAND measures a significant deficit in the $\bar{\nu}_{e}$ flux, obscrving 258 events compared to an expectation of $365.2 \pm 23.7$ cvents in the absence of oscillations. Figure 2.7 shows the $\bar{\nu}_{e}$ spectrum measured by KamLAND along with the zero oscillation expectation. The measured spectrum is analysed assuming twoflavour oscillations to determine the solar neutrino oscillation parameters $\Delta m_{12}^{2}$ and $\theta_{12}$. One important difference between the solar neutrino analysis and the KamLAND analysis is that the MSW effect has no significant influence on the propagation of reactor neutrinos through the Earth. This simplifies the determination of oscillation parameters by reducing the systematic errors. Figure 2.8(a) shows the confidence limits obtained by KamLAND for $\Delta m_{12}^{2}$ and $\theta_{12}$ along with the confidence limits obtained from the global analysis of solar neutrino data. The KamLAND confidence limits are consistent with the LMA oscillation regime and there is a clear overlap between the region of parameter space allowed by the solar neutrino analysis, and one of the regions allowed by KamLAND. Figure 2.8(b) shows the confidence limits obtained by combining the KamLAND and solar neutrino data. The best fit point is: $\Delta m_{12}^{2}=7.9_{-0.5}^{+0.6} \times 10^{-5} \mathrm{cV}^{2}$, $\tan ^{2} \theta_{12}=0.40_{-0.07}^{+0.10}$.

\subsubsection{Atmospheric Neutrinos}

High energy cosmic rays striking the atmosphere produce a cascade of secondary pions and kaons whose decay chains result in the cmission of a high flux of neutrinos. These processes can be summarised as follows (see Figure 2.9):

$$
\begin{aligned}
p, H e+N & \rightarrow X+\pi^{ \pm} / \kappa^{ \pm} \\
\pi^{ \pm} / \kappa^{ \pm} & \rightarrow \mu^{ \pm}+\nu_{\mu}\left(\bar{\nu}_{\mu}\right) \\
\mu^{ \pm} & \rightarrow e^{ \pm}+\nu_{e}\left(\bar{\nu}_{e}\right)+\bar{\nu}_{\mu}\left(\nu_{\mu}\right)
\end{aligned}
$$

The energies of atmospheric neutrinos lie in the range $E \simeq 0.1-100 \mathrm{GeV}$ and are sharply peaked toward lower energies, reflecting the energy spectrum of the incident cosmic particles. Detailed simulations have been developed which model the atmospheric neutrino flux resulting from the primary cosmic ray flux. Although uncertainties of approximately $15 \%$ exist in the absolute neutrino flux, the simulations can be used to predict neutrino flux ratios to within approximately $5 \%$. By analysing the decay 


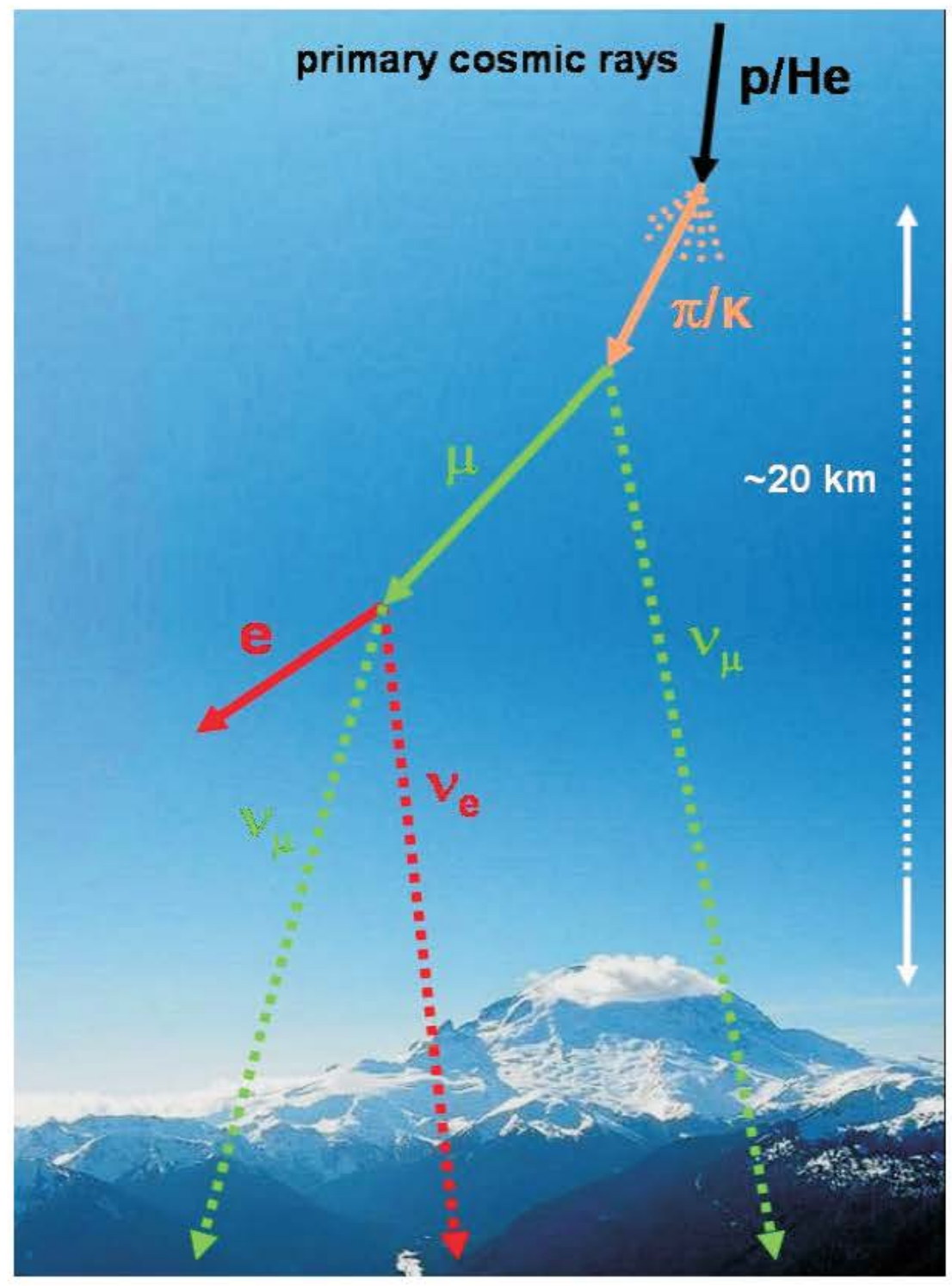

Figure 2.9: Atmospheric nentrinos ant produred by the decays of serondart particles cutated by cosmic: ray interactions in the upper atmosphere. 
chains which produce atmospheric neutrinos, the neutrino flavour ratio is predicted to be $\phi_{\mu} / \phi_{e} \approx 2$. From geometric considerations the initial flux for cach neutrino flavour is also expected to be up-down symmetric giving $\phi_{u p} / \phi_{\text {down }} \approx 1$.

The first measurements of the atmospheric $\nu_{\mu}$ and $\nu_{e}$ flux were performed in the 1980s by the IMB [41] and Kamiokande [42] experiments using water Cerrenkov detectors. Atmospheric neutrinos were detected by observing Cěrenkov rings produced by charged leptons emitted in charged current neutrino interactions. The neutrino flavour was tagged by the flavour of the emitted lepton. Electrons and muons were separated by analysing the topology of the Cerrenkov rings. Since electrons are less massive than muons they undergo more multiple scattering and therefore produce fuzzicr Cerrenkov rings. Both IMB and Kamiokande calculated the "ratio of ratios" $\Re$ between the observed and predicted neutrino flavour ratios. Both experiments observed a deficit in the $\nu_{\mu}$ flux relative to the expectation, measuring ratios of $\Re=0.54_{-0.05}^{+0.05}{ }_{-0.11}^{+0.11}$ [43] and $\Re=0.60{ }_{-0.06}^{+0.07}{ }_{-0.05}^{+0.05}[44]$ respectively.

The atmospheric $\nu_{\mu}$ and $\nu_{e}$ flux was also measured using iron calorimeter detectors. The early experiments, NUSEX [45] [46] and Frejus [47] [48], observed no evidence for a deficit in the $\nu_{\mu}$ flux. A more precise measurement was subsequently performed by the Soudan 2 experiment [49]. Soudan 2 used a $1 \mathrm{kT}$ calorimeter detector composed of active drift tubes within a passive steel structure to sample the particle tracks and showers produced by neutrino interactions. An active shield containing layers of proportional tubes was constructed around the detector to tag particles entering or exiting the detector. The signature of an atmospheric neutrino interaction was an event with a contained interaction vertex. The $\nu_{\mu} \mathrm{CC}$ and $\nu_{e} \mathrm{CC}$ interactions were separated by analysing the event topology, with $\nu_{\mu} \mathrm{CC}$ events associated with muon tracks and $\nu_{e}$ $\mathrm{CC}$ events associated electromagnetic showers. Soudan 2 observed a deficit in the $\nu_{\mu}$ flux relative to the expectation, measuring a ratio of $\Re=0.72_{-0.19}^{+0.19}{ }_{-0.07}^{+0.05}[50]$.

Neutrino oscillations offer a resolution to the atmospheric neutrino anomaly, since transitions between $\nu_{\mu}$ and other neutrino flavours result in a deficit in the measured $\nu_{\mu}$ flux relative to the expectation. One sensitive method of detecting the presence of neutrino oscillations in atmospheric neutrinos is to measure the zenith angle distribution of the $\nu_{\mu}$ flux. The zenith angle is defined as the angle between the incident neutrino direction and the vertical. For a detector close to the surface of the Earth, atmospheric neutrinos are characterized by a wide variation in the propagation distance $L$ between 
the neutrino production and detection points. Down-going neutrinos cover distances of $L \simeq 20-500 \mathrm{~km}$, whereas 11p-going neutrinos cover distances of $L \simeq 500-13,000$ $\mathrm{km}$. If neutrino oscillations occur at wavelengths within this range of distances, then deficits in the measured $\nu_{\mu}$ flux will occur at angles where the neutrino propagation distance corresponds to a high probability of neutrino oscillations. Recently, compelling evidence in favour of neutrino oscillations has been observed in atmospheric neutrinos by the Supcr-Kamiokande experiment.

\subsubsection{Super-Kamiokande}

SKK has performed precise measurements of the atmospheric $\nu_{\mu}$ and $\nu_{\epsilon}$ flux. The large fiducial volume of the SKK detector enables a large sample of multi-GeV charged current neutrino interactions to be acquired. These events provide a high sensitivty to neutrino oscillations, since the emitted lepton closely follows the path of the incident neutrino, giving an accurate measurement of the neutrino propagation distance. The atmospheric neutrino events are divided into a number of classes: Fully Contained (FC) events, in which the neutrino interaction vertex and emitted particles are contained within the fiducial volume; Partially Contained (PC) events, in which the interaction vertex is contained but one or more particles are observed to exit the detector; and neutrinoinduced Lp-Going Muons (UPMU), in which newtrinos interact in the rock below the detector to produce muons that then enter the detector.

SKK has published an analysis of atmospheric neutrino data acquired between April 1996 and November 2001 [51]. Figure 2.10 shows the angular distribution measured the SKK event classes and energy samples. The multi-GeV $\nu_{\mu}$ distributions dip to approximatcly half the expected flux at large zenith angles. In contrast, the $\nu_{e}$ distributions are in good agreement with the expected flux. The interpretation of this data is that atmospheric neutrinos undergo $\nu_{\mu} \leftrightarrow \nu_{\tau}$ oscillations at close to maximal mixing. The angular distributions are analysed to determine the parameters $\Delta m_{23}^{2}$ and $\theta_{23}$ that control short range $\nu_{\mu} \leftrightarrow \nu_{\tau}$ oscillations. Figure 2.11 shows the confidence limits obtained by the SKK analysis. The $90 \%$ confidence limits are: $1 . \bar{j} \times 10^{-3}<\Delta m_{23}^{2}<3.4 \times 10^{-3} \mathrm{eV}^{2}$, $\sin ^{2} 2 \theta_{23}>0.92$. The best fit point is: $\Delta m_{23}^{2}=2.1 \times 10^{-3} \mathrm{cV}^{2}, \sin ^{2} 2 \theta_{23}=1.0$.

The SKK has published a separate $L / E$ analysis [52]. The mean $L / E$ resolution is calculated as a function of the reconstructed neutrino energy and zenith angle. Regions with a resolution of $<70 \%$ are used in the oscillation analysis. Regions with a resolution 

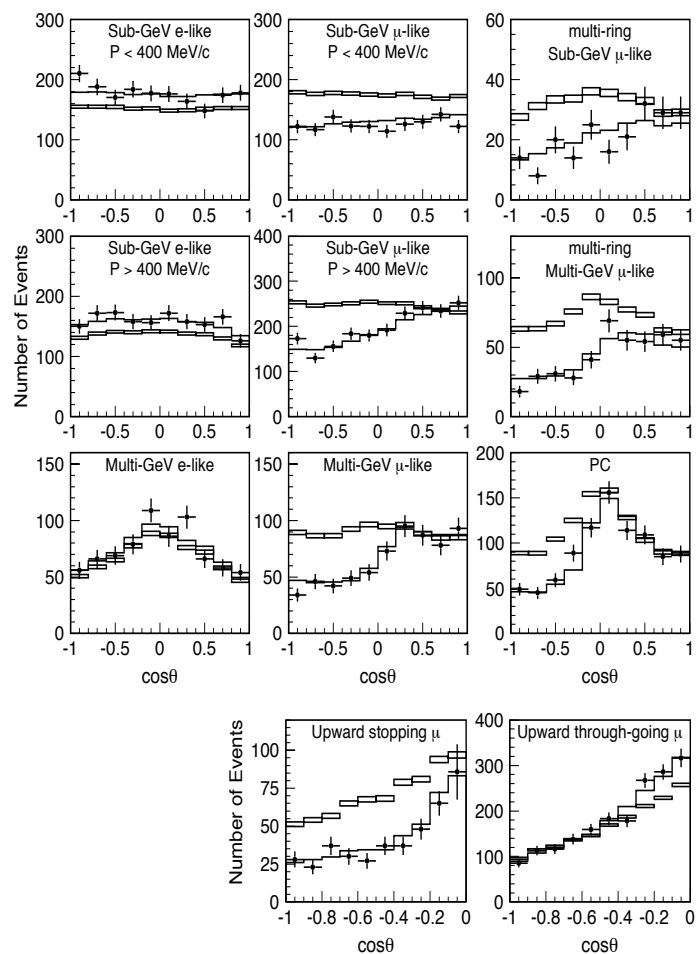

Figure 2.10: Super-Kamiokande zenith angle distribution. The data is represented by the points, the zero oscillation predictions by the boxes, and the best fit $\nu_{\mu} \leftrightarrow \nu_{\tau}$ oscillations by the solid lines [51].

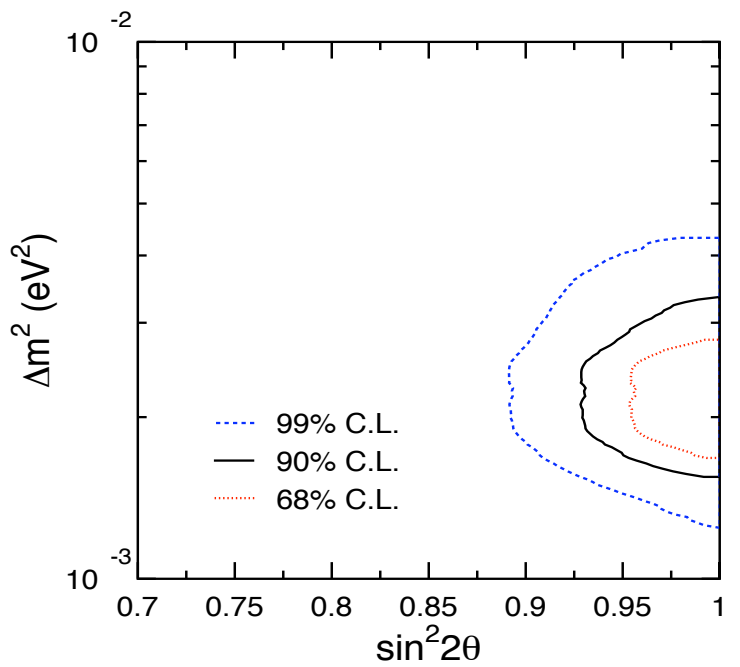

Figure 2.11: SKK allowed region for $\nu_{\mu} \leftrightarrow \nu_{\tau}$ oscillations [51]. 


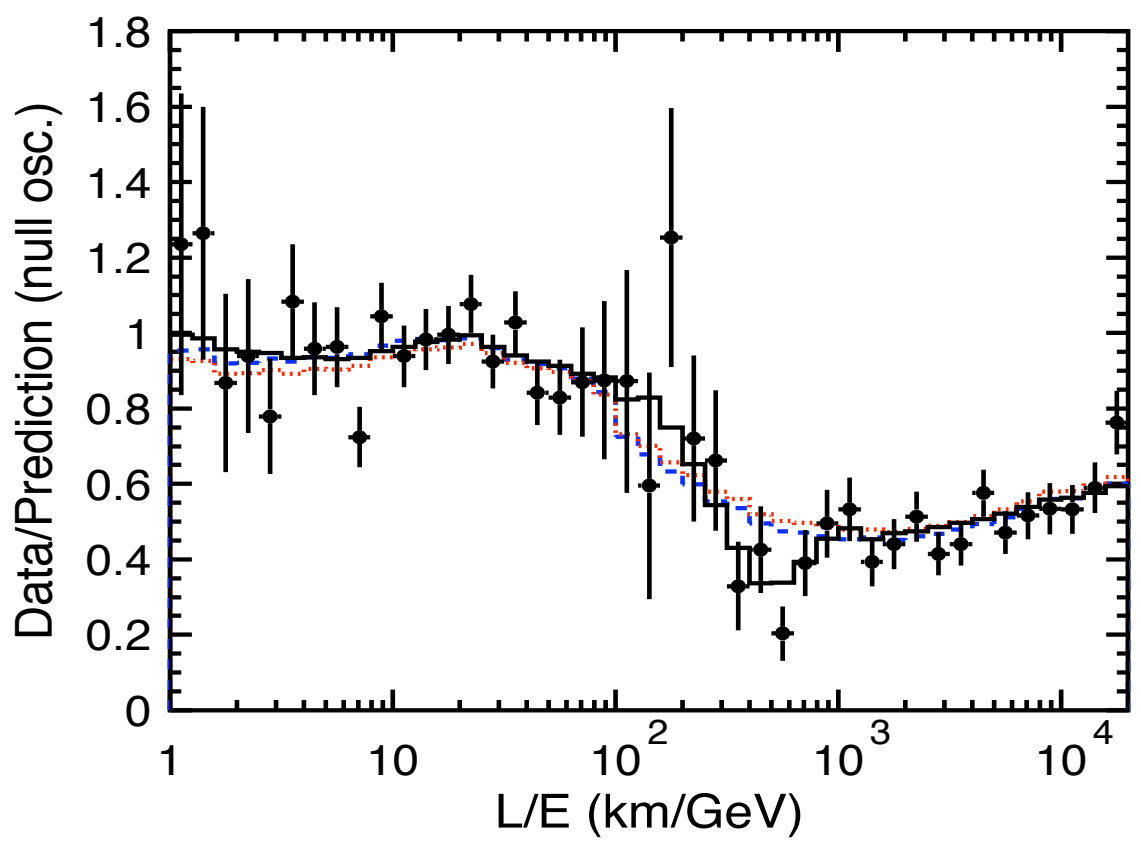

Figure 2.12: Super-Kamiokande L/E analysis. The ratio between the measured $L / E$ distribution and the expectation in the absence of oscillations is shown. The best fit to neutrino oscillations is represented by the solid line; the best fit to neutrino decay is represented by dashed line; the best fit to neutrino neutrino decoherence is represented by the dotted line [52].

of $>70 \%$ occur close to the horizon where the propagation distance varies rapidly with zenith angle, and at low energies where the mean angle between the neutrino and muon is large. Figure 2.12 shows the ratio between the measured $L / E$ distribution and the expectation for no oscillations. A dip is observed at $L / E \simeq 500 \mathrm{~km} / \mathrm{GeV}$, consistent with the expectation that that oscillation probability should be periodic with $L / E$. The distribution is analysed to determine the oscillation parameters $\Delta m_{23}^{2}$ and $\theta_{23}$ assuming two-flavour oscillations. The $90 \%$ confidence limits are: $1.9 \times 10^{-3}<$ $\Delta m_{23}^{2}<3.0 \times 10^{-3} \mathrm{eV}^{2}, \sin ^{2} 2 \theta_{23}>0.90$. The best fit point inside the physical region is: $\Delta m_{23}^{2}=2.1 \times 10^{-3} \mathrm{eV}^{2}, \sin ^{2} 2 \theta_{23}=1.0$. This is consistent with the confidence limits obtained by the analysis of the neutrino zenith angle distributions. The $L / E$ analysis also disfavours alternative oscillation models including neutrino decay [53] [54] and neutrino decoherence [55] [56] that explain the distortion of the atmospheric neutrino flux with zenith angle but do not predict any dip in the $L / E$ distribution. 


\subsubsection{K2K}

The oscillation signal observed in atmospheric neutrinos has recently been confirmed by the $\mathrm{K} 2 \mathrm{~K}$ long baseline accelerator neutrino experiment [57]. K2K uses an accelerator $\nu_{\mu}$ beam to study neutrino oscillations. The neutrino beam is manufactured by the KEK accelerator facility in Tsukuba, Japan. A beam of protons from the $12 \mathrm{GeV}$ synchrotron at KEK is fired onto a fixed aluminium target producing an intense flux of secondary pions and kaons. The positively charged secondaries are focused by a pair of magnetic horns and then directed into a $200 \mathrm{~m}$ long decay pipe where they decay to produce a $98 \%$ pure $\nu_{\mu}$ beam with a mean energy of $1.3 \mathrm{GeV}$. The initial unoscillated $\nu_{\mu}$ spectrum is measured at a distance of $300 \mathrm{~m}$ from the proton target using a $1 \mathrm{kT}$ water Cecrenkov detector and a system of fine-grained detectors. The beam travels $250 \mathrm{~km}$ through the Earth and is then measured by the Super-Kamiokande detector. The beam is produced in pulses, enabling neutrino interactions in the SKK detector to be identified using timing information. The signature of neutrino oscillations is a distortion in the neutrino energy spectrum at SKK relative to the expectation in the absence of oscillations. The expectation for no oscillations is determined by extrapolating the neutrino flux measured by the two near detectors to the SKK detector. The beam parameters of $E \simeq 1 \mathrm{GeV}$ and $L \simeq 250 \mathrm{~km}$ provide sensitivity to oscillations of order $\Delta m^{2} \simeq 10^{-3} \mathrm{cV}^{2}$.

K2K has published an analysis of beam data acquired between June 1999 and February 2004 [58]. K2K measures a deficit of events at SKK, observing 107 events compared with an expectation of $151_{-10}^{+12}$ events in the absence of oscillations. A sample of 57 single-ring $\mu$-like events is chosen to measure the energy spectrum of the beam. For these events, the neutrino energics are reconstructed from the measured encrgy and direction of the emitted muons assurning charged current quasi-elastic kinematics. A two-flavour oscillation analysis is applied to the neutrino energy spectrum to determine the parameters $\Delta m_{23}^{2}$ and $\theta_{23}$. Figure 2.13 shows the cnergy spectrum along with the zero oscillation prediction and the best fit to neutrino oscillations. There is a clear spectral distortion in the measured events. Figure 2.14 shows the confidence limits obtained by the K2K analysis. The $90 \%$ confidence limits on $\Delta m_{23}^{2}$ are: $1.9 \times 10^{-3}<\Delta m_{23}^{2}<3.6 \times 10^{-3} \mathrm{cV}^{2}$ The best fit point inside the physical region is: $\Delta m_{23}^{2}=2.8 \times 10^{-3} \mathrm{eV}^{2}, \sin ^{2} 2 \theta_{23}=1.0$. The confidence limits obtained by the $\mathrm{K} 2 \mathrm{~K}$ experiment are consistent with those obtained by the the SKK experiment. 


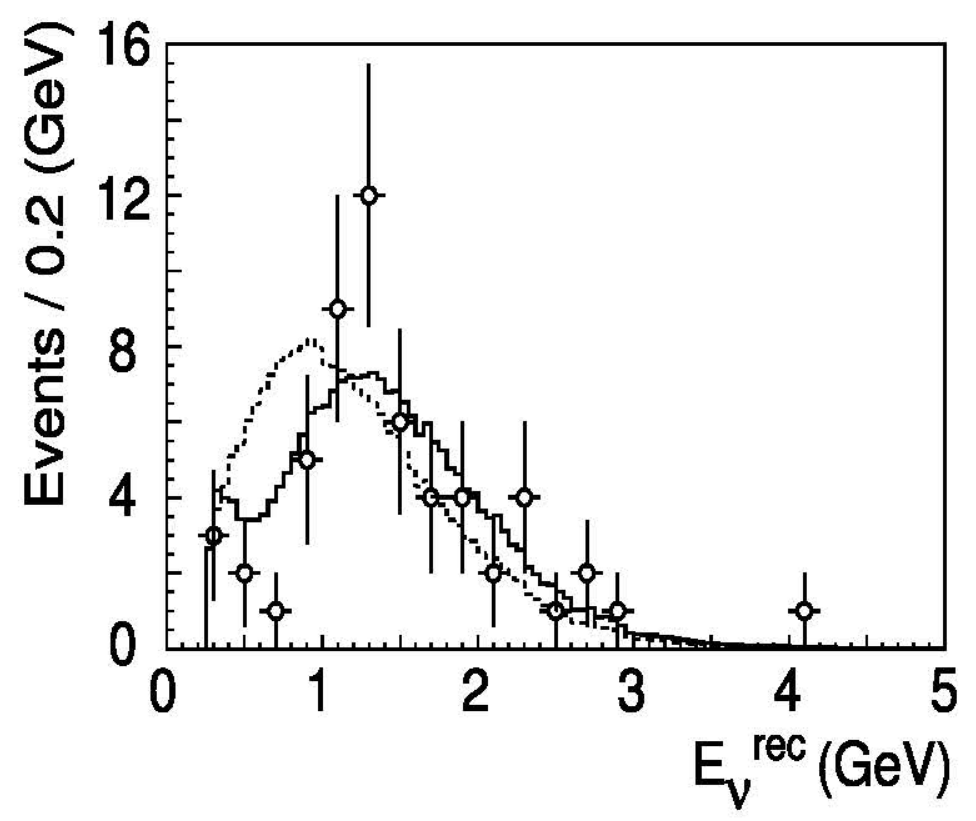

Figure 2.13: The reconstructed energy spectrum of $\nu_{\mu} C C$ events measured by K2K. The data is represented by the points, the zero oscillation spectrum by the dotted line, and the best fit oscillations by the solid line [58].

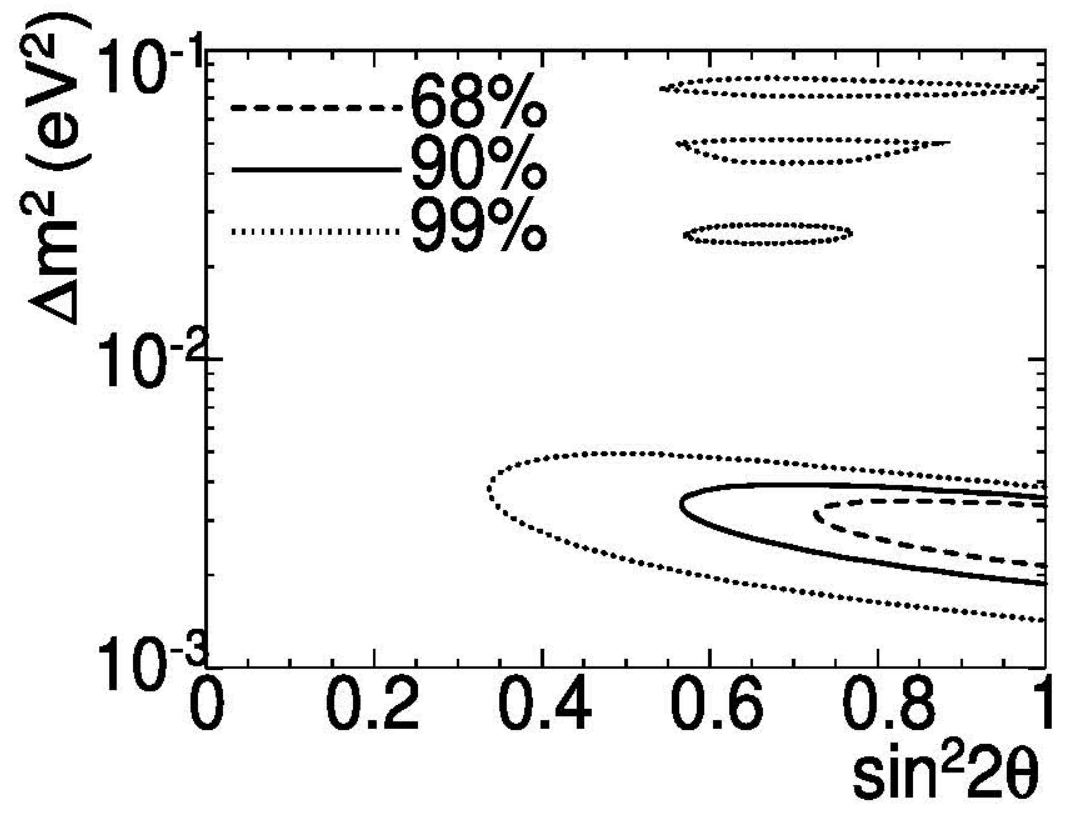

Figure 2.14: K2K allowed region for $\nu_{\mu} \leftrightarrow \nu_{\tau}$ oscillations [58]. 


\subsubsection{CHOOZ}

The solar and atmospheric neutrino oscillation sectors are weakly coupled by a small mixing angle $\theta_{13}$. This mixing angle can be measured by detecting the sub-dominant $\nu_{e} \leftrightarrow \nu_{\mu} / \nu_{\tau}$ oscillation mode at distances associated with atmospheric neutrino oscillations. The current generation of experiments has set an upper limit on the value of $\theta_{13}$. The tightest bound on $\theta_{13}$ has been set by the CHOOZ reactor neutrino experiment [ 59 ].

CHOOZ was a short baseline reactor neutrino experiment located $1 \mathrm{~km}$ from the CHOOZ nuclear power plant in northern France. CHOOZ measured the flux of emitted $\bar{\nu}_{e}$ using a $\bar{\jmath}$ ton liquid scintillator detector. The $\bar{\nu}_{e}$ were detected by the inverse beta decay process $\bar{\nu}_{e}+p \rightarrow n+e^{+}$. The signature of a $\bar{\nu}_{e}$ interaction was a prompt $e^{+}$signal followed by photon emissions from the capture of the $n$. The CHOOZ detector was loaded with Gadolinium to enhance the efficiency of neutron capture and the energy of the photon emissions following neutron capture.

The signature of oscillations at $\mathrm{CHOOZ}$ was a deficit in the measured rate of $\bar{\nu}_{e}$ and a distortion in the $\bar{\nu}_{e}$ spectrum relative to the zero oscillation prediction. Figure 2.15 shows the prompt $e^{+}$spectrum measured by CHOOZ along with the expectation in the abscnec of oscillations. The measured spectrum shows no significant deficit or spectral distortion. Figure 2.16 shows the confidence limits on $\theta_{13}$ as a function of $\Delta m_{23}^{2}$ obtained assuming two-flavour oscillations. The $90 \%$ confidence limit is $\sin ^{2} 2 \theta_{13}<0.17$ [60].

\subsubsection{LSND}

An additional unconfirmed oscillation signal has been reported by the LSND short baseline accelerator neutrino experiment located at the Los Alamos National Laboratory in New Mexico [61]. LSND measured a beam of neutrinos produced by a beam dump of $800 \mathrm{MeV}$ protons onto a fixed target. The secondary $\pi^{-}$are mostly absorbed by the target, but the sccondary $\pi^{+}$mostly come to rest and decay by $\pi^{+} \rightarrow \mu^{+} \nu_{\mu}$. Most of the emitted muons then also come to rest and decay by $\mu^{+} \rightarrow e^{+} \nu_{e} \bar{\nu}_{\mu}$. Since the majority of decays take place at rest, the neutrino beam has a well defined energy spectrum with a maximum energy of $E \simeq 50 \mathrm{McV}$. LSND used a 160 ton liquid scintillator detector located $30 \mathrm{~m}$ away from the beam production point to search for $\bar{\nu}_{e}$ appearance.

LSND reported an excess of $87.9 \pm 22.4 \pm 6.0 \bar{\nu}_{e}$ events in the range $E \simeq 36-60 \mathrm{MeV}$, interpreted as being the result of $\bar{\nu}_{\mu} \leftrightarrow \bar{\nu}_{e}$ oscillations [62]. However, other short baseline 


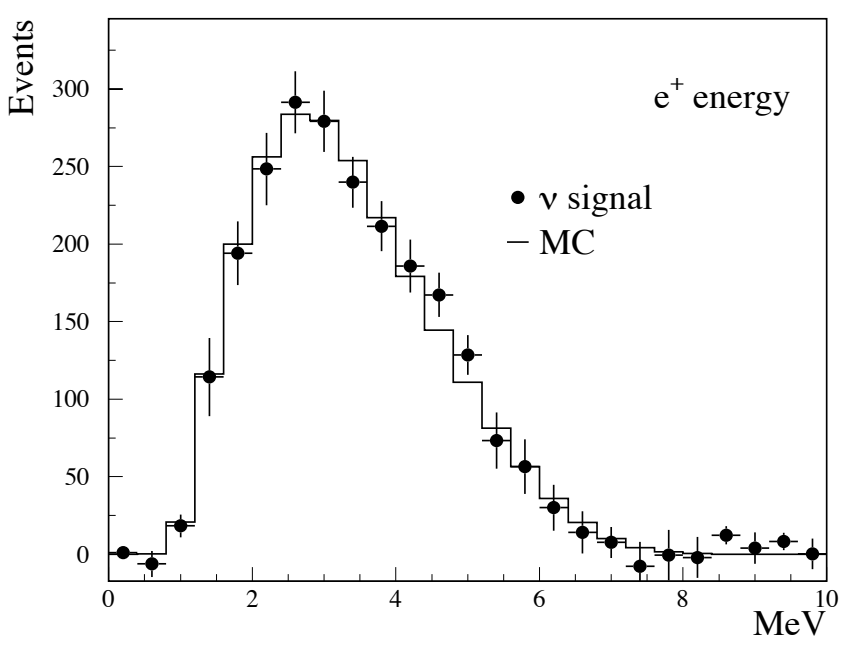

Figure 2.15: CHOOZ prompt $e^{+}$spectrum [60]. The measured energy spectrum is represented by the points; the expectation in the absence of oscillations is represented by the solid line.

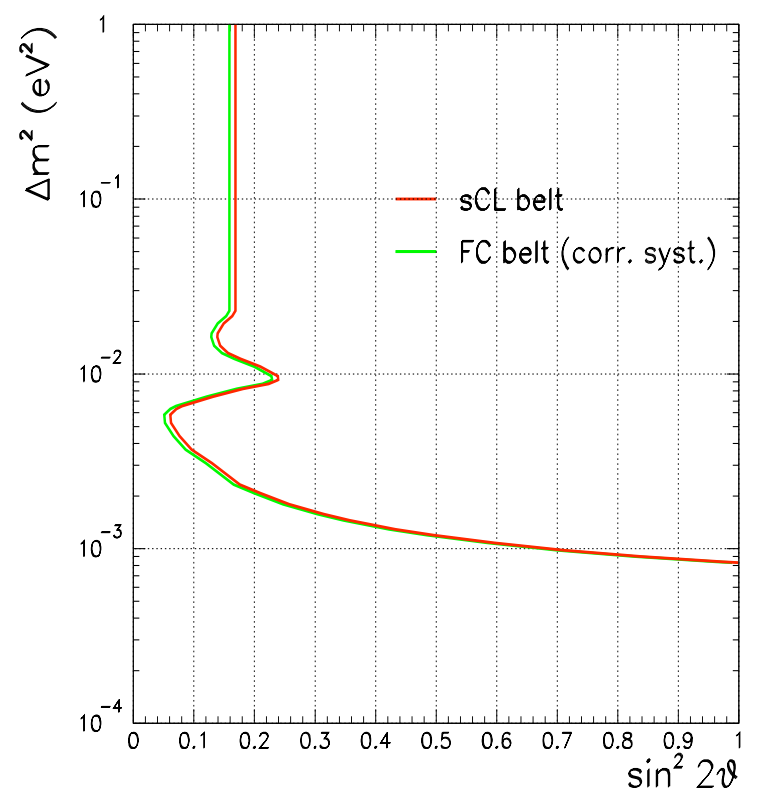

Figure 2.16: $C H O O Z$ confidence limits on $\sin ^{2} 2 \theta_{13}$ and $\Delta m_{23}^{2}$. The region to the right of the line is excluded at $90 \%$ confidence. The two lines correspond to different methods of obtaining the 90\% confidence interval [60]. 


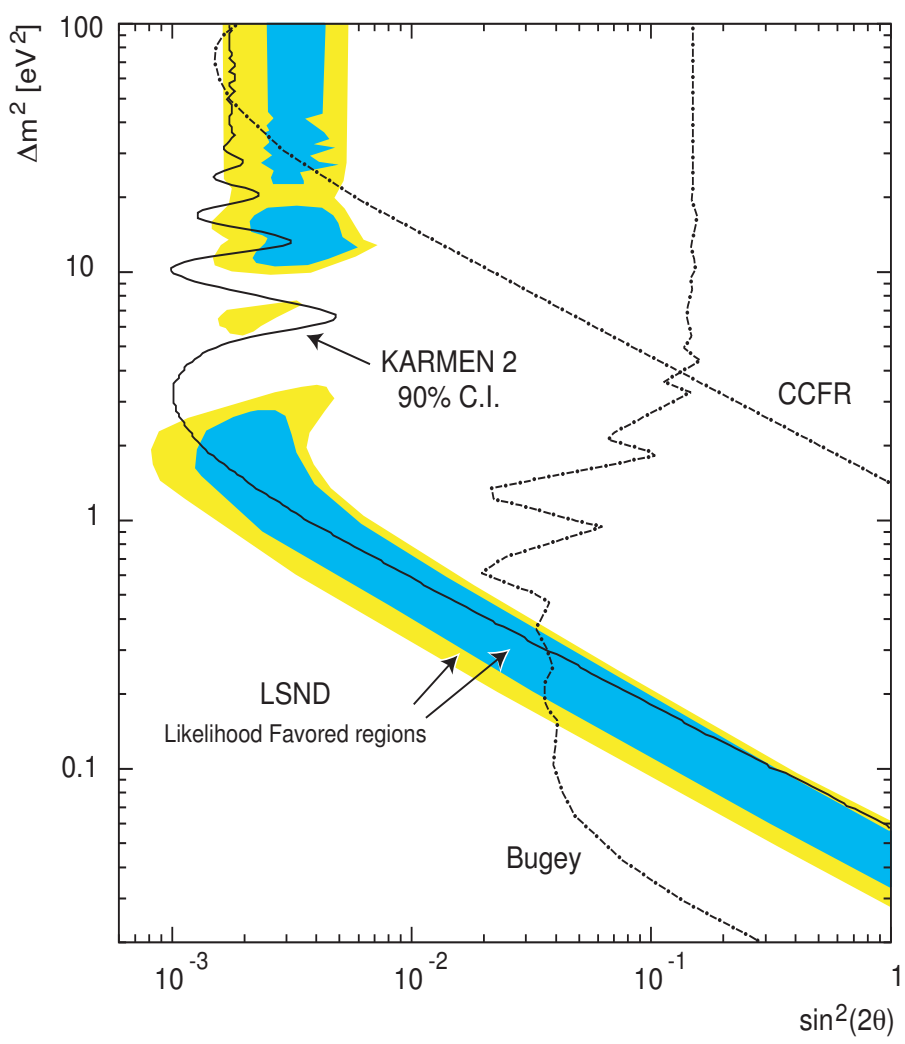

Figure 2.17: Comparison of results from short baseline experiments [63].

neutrino oscillation experiments have not observed a similar $\bar{\nu}_{e}$ appearance signal. Both the KARMEN 2 [63] accelerator neutrino and BUGEY [64] reactor neutrino experiments have observed $\bar{\nu}_{e}$ rates in good agreement with the expectation. Figure 2.17 shows the confidence limits obtained from these experiments assuming two-flavour oscillations. Much of the LSND region is excluded, but two regions at $\Delta m^{2} \simeq 0.2-1 \mathrm{eV}^{2}$ and $\Delta m^{2} \simeq 7 \mathrm{eV}^{2}$ remain consistent with all the data.

If LSND is correct, it represents a third $\Delta m^{2}$ measurement. It is not possible to define three independent $\Delta m^{2}$ values using three neutrino flavours, so to explain the LSND result it is necessary to introduce additional "sterile" neutrinos which do not couple to the weak force normally [65] or invoke exotic solutions such as CPT violation [66]. It is important to obtain independent verification of the LSND result. To this end, the MiniBooNE experiment has been constructed at Fermilab [67]. This is sensitive to neutrino oscillations over the entire LSND signal region. 


\subsection{The Current Status}

The current generation of neutrino experiments has observed strong evidence for mixing between neutrino flavours. These experiments have determined the general form of the neutrino mixing matrix. There are two confirmed oscillation signals which provide measurements of two neutrino mass splittings and two mixing angles. The results of solar neutrino experiments and KamLAND are interpreted as $\nu_{e} \leftrightarrow \nu_{\mu} / \nu_{\tau}$ oscillations with $\Delta m_{12}^{2} \simeq 10^{-4} \mathrm{cV}^{2}$ and $\theta_{12} \simeq 30^{\circ}$. The results of atmospheric neutrino experiments and $\mathrm{K} 2 \mathrm{~K}$ are interpreted as $\nu_{\mu} \leftrightarrow \nu_{\tau}$ oscillations with $\Delta m_{23}^{2} \simeq 10^{-3} \mathrm{eV}^{2}$ and $\theta_{23} \simeq 45^{\circ}$. The third mixing angle is constrained by CHOOZ to be in the region $\theta_{13} \leq 10^{\circ}$. In the limit that $\theta_{13} \approx 0$, the mixing matrix can be written as follows:

$$
\left(\begin{array}{c}
\nu_{e} \\
\nu_{\mu} \\
\nu_{\tau}
\end{array}\right) \approx\left(\begin{array}{ccc}
0.83 & 0.56 & 0 \\
-0.40 & 0.59 & 0.71 \\
0.40 & -0.59 & 0.71
\end{array}\right)\left(\begin{array}{c}
\nu_{1} \\
\nu_{2} \\
\nu_{3}
\end{array}\right)
$$

The LSND experiment provides evidence of a third neutrino oscillation signal which cannot be incorporated in the above picture. Confirmation of the LSND result would indicate the existence of additional sterile neutrino flavours or point towards some other exotic form of new physics. The MiniBooNE experiment has been constructed to resolve this issue and will confirm or refute the LSND signal.

The current experiments have led to significant advances in neutrino physics but have also raised new questions on the nature of neutrino mass and mixing:

- Do neutrinos really oscillate? Neutrino oscillations are predicted to have a periodic dependence on $L / E$. The current generation of experiments have observed hints of a periodic structure in $L / E$. However no clear-cut observation has yet been made.

- How big is the angle $\theta_{13}$ ? A measurement of the mixing angle $\theta_{13}$ would fill in a missing part of the neutrino mixing matrix. The size of this mixing angle also determines the extent of $\mathrm{CP}$ violation in the neutrino sector and could have important consequences in cosmology [68] [69]. The current generation of experiments have only set an upper limit on the value of $\theta_{13}$. 
- What is the sign of $\Delta \mathbf{m}_{23}^{2}$ ? Since the transition probabilities of neutrino oscillations in a vacu1um do not depend on the sign of the neutrino mass splitting, it is currently not known whether neutrinos occupy a nor mal mass hierarchy in which $\Delta m_{23}^{2}>0$, or an inverted mass hicrarchy in which $\Delta m_{23}^{2}<0$. The degeneracy can be broken by exploiting matter effects in long baseline neutrino and anti-neutrino oscillations.

- Is the angle $\theta_{\mathbf{2 3}}$ maximal ? The mixing angle $\theta_{23}$ is known to lie around $45^{\circ}$, but the allowed range of angles is large. Precise measurements are required to determine the value of $\theta_{23}$ and any possible deviation from maximal mixing.

These questions provide the motivation for a new generation of experiments that will perform precise measurements of neutrino oscillations. The MINOS experiment is a part of this new generation. MINOS is a long baseline neutrino oscillation experiment conducting a precision study of oscillations in an accelerator $\nu_{\mu}$ beam. MINOS will definitively test the neutrino oscillation hypothesis by searching for the expected periodicity in $\nu_{\mu} \leftrightarrow \nu_{\tau}$ oscillations, and will precisely determine the oscillation parameters $\Delta m_{23}^{2}$ and $\theta_{23}$ by measuring these oscillations. MINOS will also determine the value of $\theta_{13}$ or set a new limit by searching for $\nu_{\mu} \leftrightarrow \nu_{e}$ oscillations. In addition MINOS will conduct the first separate measurements of $\nu_{\mu} \leftrightarrow \nu_{\tau}$ and $\bar{\nu}_{\mu} \leftrightarrow \bar{\nu}_{\tau}$ oscillations in atmospheric neutrinos. 


\section{Chapter 3}

\section{The MINOS Experiment}

\subsection{MINOS Overview}

The Main Injector Neutrino Oscillation Search (MINOS) [70] is a long bascline experiment conducting a study of oscillations in an accelerator beam of muon neutrinos. MINOS aims to confirm the existence of neutrino oscillations and perform precise measurements of the oscillation parameters.

The MINOS beam line is illustrated in Figure 3.1. The neutrino beam is manufactured at the Fermi National Accelerator Laboratory in Illinois, USA. The neutrino energy spectrum is sampled at two points along the beam line using two functionally similar sampling calorimeters: a $1 \mathrm{kT}$ Near Detector constructed $1 \mathrm{~km}$ away from the proton target at Fermilab; and a $5 \mathrm{kT}$ Far Detector located $730 \mathrm{~km}$ away at the Soudan Lnderground Laboratory in Minnesota, USA. A search for neutrino oscillations is conducted by comparing the cnergy spectrum measured by each detector. The Near Detector spectrum is used to make a prediction of the Far Detector spectrum in the absence of neutrino oscillations. This is then compared with the spectrum measured by the Far Detector. The signature of oscillations is a deficit of muon neutrinos in the Far Detector spectrum with a periodic energy dependence.

In addition, the Far Detector can be used to measure atmospheric neutrino oscillations [71] [72]. Its large mass produces a sufficient event rate and its location decp underground provides a good degree of shielding against the large flux of cosmic ray muons incident on the Earth's surface. The Far Detector is unique in being the first massive underground detector to possess a magnetic field. This makes the separation of 

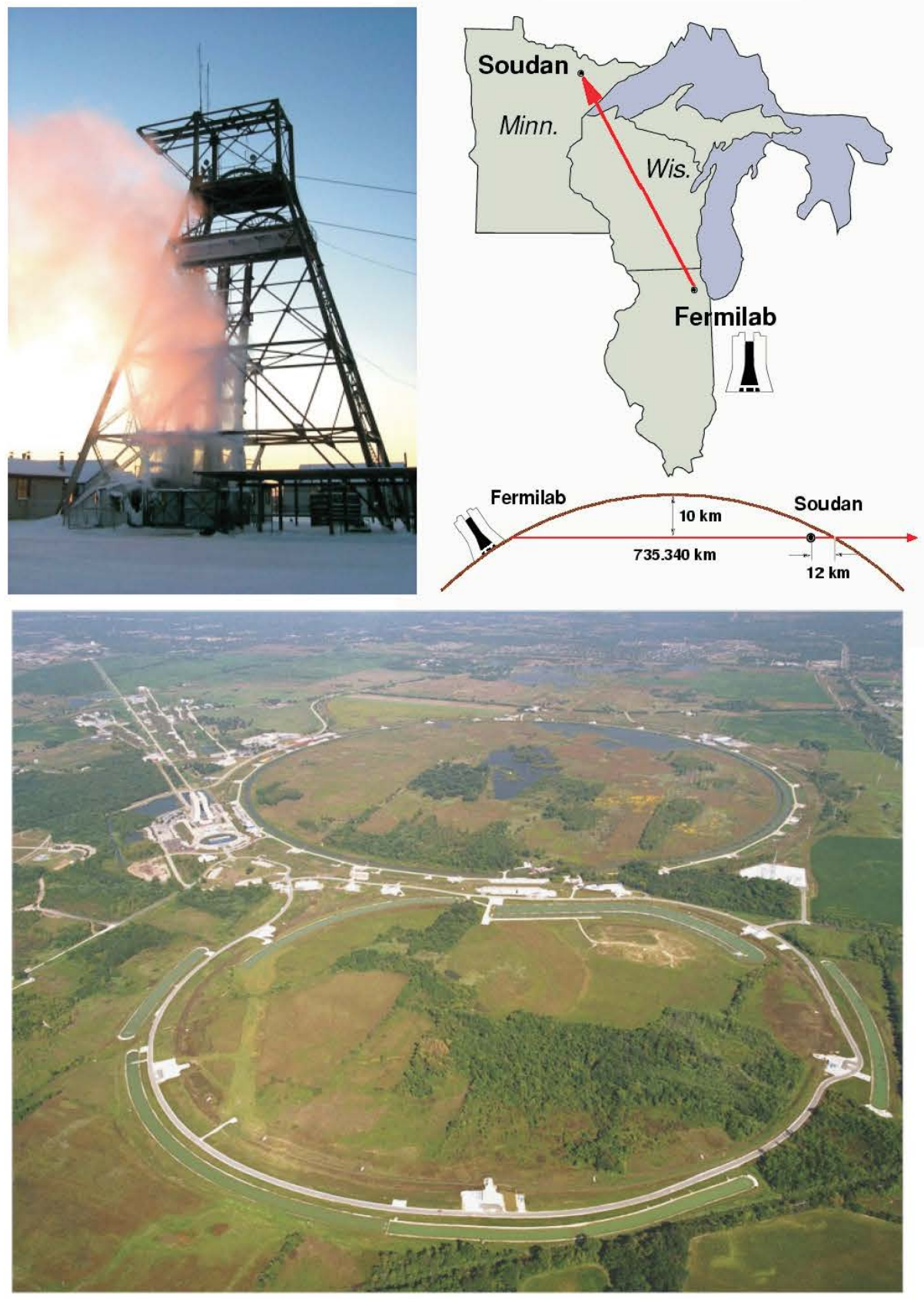

Figure 3.1: The MINOS neutrino beam line. The neutrinos originate at the Fermi Laboratory in Illinois (bottom) and then travel $7.35 \mathrm{~km}$ through the Earth to the Soudan Underground Laboratory in Minnesota (top left). 
atmospheric neutrinos and anti-neutrinos possible, enabling separate measurements of oscillations in neutrinos and anti-neutrinos to be carried out for the first time.

MINOS is a large international collaboration of 175 physicists from 32 institutions in 6 countries. The experiment has recently commenced full opcrations. The Far Detector was completed in August 2003 and has now collected a large set of atmospheric neutrino and cosmic ray data. The Near Detector was completed in August 2004 and the accelerator beam became fully opcrational in March 2005.

\subsection{The NuMI Beam}

The MINOS neutrino beam is produced by the dedicated Main Injector Neutrino (NuMI) beam facility based at Fermilab [73]. The layout of the NuMI beam line is set out in Figure 3.2. A sequence of three kicker magnets is used to rapidly extract batches of 120 GoV protons from the Main Injector Ring and direct them into the N11MI beam line. The beam spills are $8 \mu \mathrm{s}$ in duration, spaced $1.9 \mathrm{~s}$ apart, and each contain $2.5 \times 10^{13}$ protons (at design intensity). The protons are transported along a carricr tunnel and then directed onto a $1 \mathrm{~m}$ long, $6.4 \mathrm{~mm}$ wide, $18 \mathrm{~mm}$ high segmented graphite target. The protons interact in the target to produce secondary particles, consisting mainly of pions and kaons. The long and narrow shape of the target is chosen to cnable the majority of protons to interact in the target but also allow the secondary pions and kaons to escape through the sides of the target thus minimizing any re-absorption by the target.

The secondary particles emitted from the target are focused back onto the axis of the primary proton beam using two magnetic focusing horns (see Figure 3.3). Each magnetic horn consists of an inner and outer cylindrical conductor. Pulses of $200 \mathrm{kA}$ current are applied to the conductors in time with the beam spills, generating a toroidal magnetic field between the conductors. The inner conductors are parabolic in shape so that the horns act as lenses and focus charged particles incident on the horns between the conductors. The direction of the current is chosen to focus positively charged particles. The focal length of each magnetic horn is approximately proportional to the momentum of the incident particles. This means that the momentum of the focused secondaries and thus the neutrino spectrum is controlled by the relative position of the two magnetic horns and the target. To select secondary pions and kaons with low momentum, the horms are positioned close to the target; and to select secondaries with high momentum, 


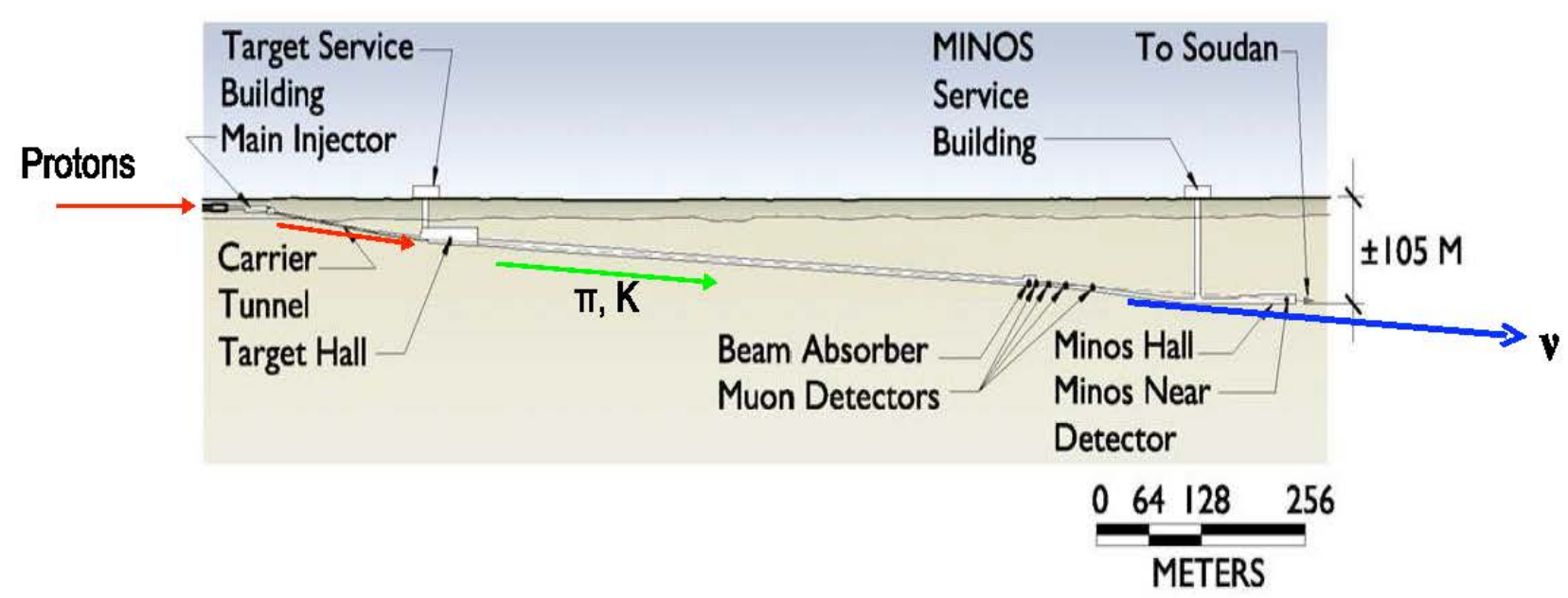

Figure 3.2: Layout of the NuMI bearn line at Fermilab. A beam of $120 \mathrm{GeV}$ protons is directed onto the graphite target. The secondary pions and kaons are then focused by a pair of magnetic horns into a $675 \mathrm{~m}$ long decay pipe where they decay to produce a beam of muon neutrinos.

the horns are positioned away from the target.

The focused beam of secondary pions and kaons is directed down a $675 \mathrm{~m}$ long, $2 \mathrm{~m}$ diameter decay pipe. The pions and kaons undergo $\pi^{+} \rightarrow \mu^{+} \nu_{\mu}$ and $K^{+} \rightarrow \mu^{+} \nu_{\mu}$ decays to produce a beam of $\nu_{\mu}$. The beam also contains small components $(<2 \%)$ of $\bar{\nu}_{p}$ and $\nu_{e}$ produced by the secondary decay $\mu^{+} \rightarrow e^{-} \bar{\nu}_{k} \nu_{e}$. At the end of the decay pipe, any remaining hadrons are stopped by a metal absorber. The muons produced by the decays of pions and kaons then range out in the $240 \mathrm{~m}$ of rock that lies between the ond of the decay pipe and the Near Detector, leaving a pure beam of noutrinos.

The profile of the hadrons and muons at the end of the decay pipe is monitored using an array of ionization detectors [71]. A hadron monitor is constructed in front of the absorber at the end of the decay pipe to measure pions, kaons and protons. Three muon detectors are constructed in alcoves dug into the rock $10-50 \mathrm{~m}$ downstream of the absorber to measure the profile of mons enitted in the decays of pions and kaons. The lateral profiles of muons and hadrons provide a good measure of the integrity of the NuMI target and the focusing of the neutrino beam.

The NuMl beam line has been designed to provide a high degree of flexibility in the choice of neutrino beam spectrum. The shape and energy range of the spectrum is controlled by the configuration of the horns and the target. Three possible target- 


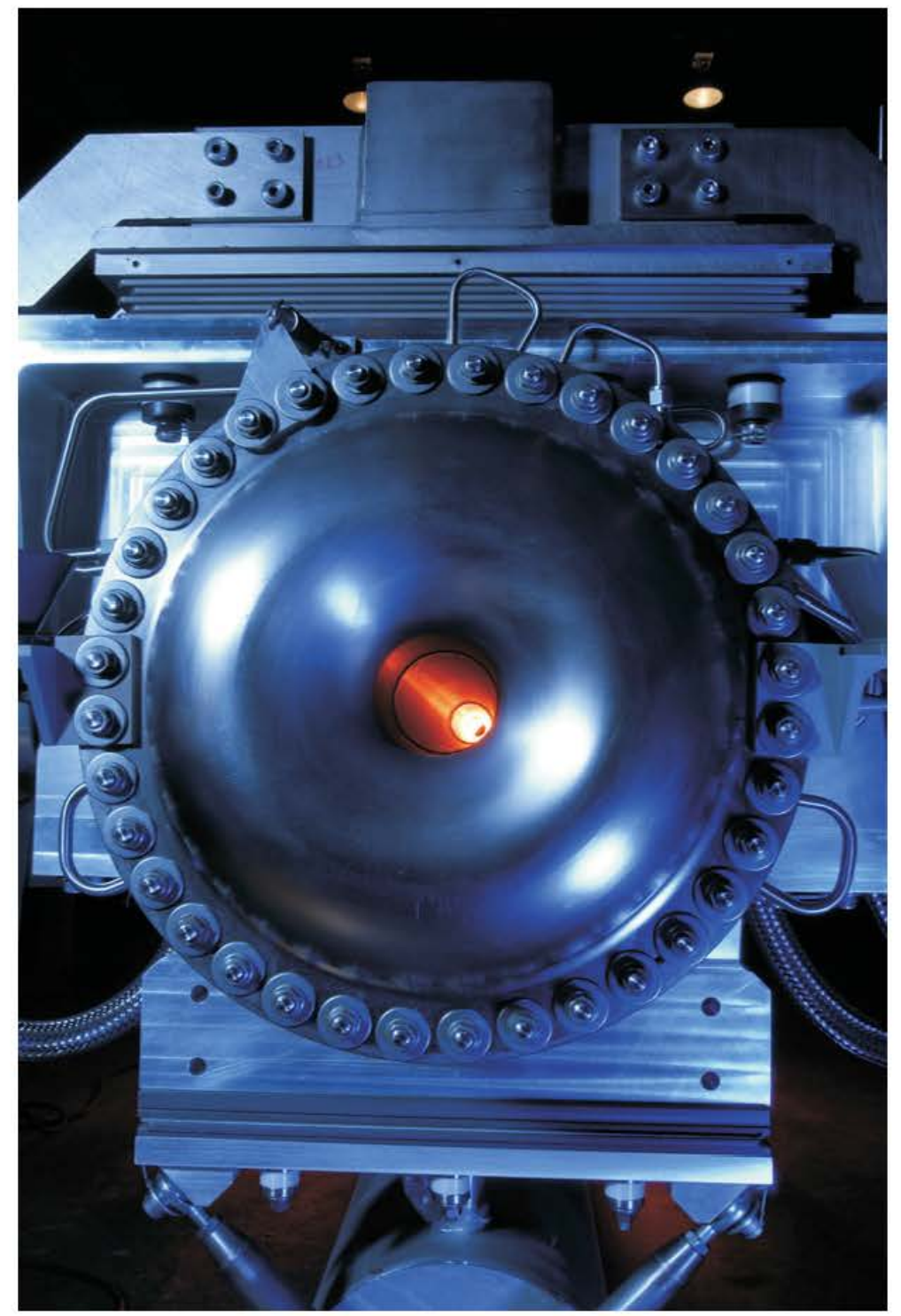

Figure 3.3: View through one of the two NuMI focusing horns. Pulses of current are applied in time with the beam spills generaling a magnelic field. which is used to focus secondary particles onto the axis of the beam line. 


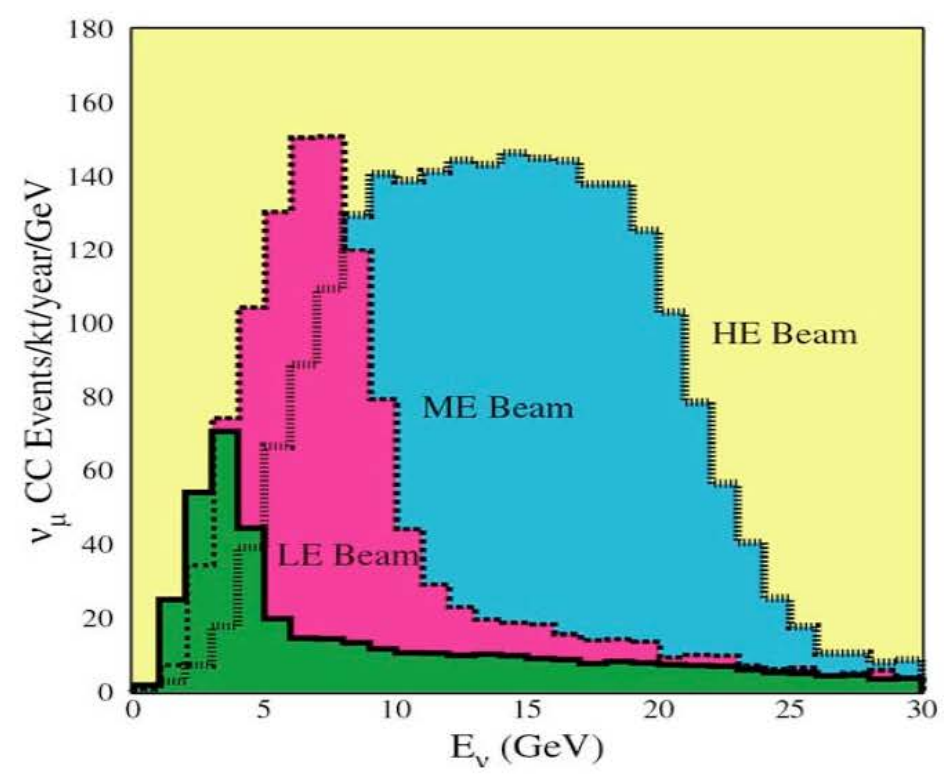

Figure 3.4: The projected energy spectrum of $\nu_{\mu} C C$ events at the Far Detector for the proposed $L E, M E$ and $H E$ target-horn configurations.

horn configurations have been proposed for use in MINOS which cover the full range of possible neutrino energies:

- High Energy (HE) A beam with $E_{y} \simeq 5-25 \mathrm{GeV}$ is obtained by positioning the two horms at their maximum separation and placing the target at the maximum distane from the first horn.

- Medium Energy (ME) A beam with $E_{\nu} \simeq 3-13 \mathrm{GeV}$ is obtained by bringing the horns closer together and moving the target towards the first horn.

- Low Energy (LE) A beam with $E_{\nu} \simeq 1-5 \mathrm{GeV}$ is obtained by positioning the horns adjacent to each other and moving the target inside the first horn.

Figure 3.4 shows the projected energy spectrum of $\nu_{\mu}$ CC events at the Far Detector for each proposed target-horn configuration after 1 year (at design intensity). As the peak encrgy of the beam is reduced, the overall flux intensity at the Far Detector also decreases. The beam is initially being run in the LE configuration since this maximizes the expected neutrino interaction rate at neutrino energies corresponding to the region of oscillations favoured by the SKK experiment. 


\subsection{The MINOS Detectors}

The MINOS experiment uses two detectors to measure the neutrino beam. The detectors are designed to be as functionally similar as possible in order to reduce the systematic crrors in the oscillation measurement. Both detectors are sampling calorimeters composed of interleaved layers of steel and plastic scintillator. Neutrinos interact in the steel to produce a number of secondary particles. Charged particles passing through the scintillator generate small quantities of light which are amplified by photo-multiplier tubes and read out by sensitive electronics. The detectors are magnetized by current-carrying coils which generate a toroidal magnetic field in the steel planes. This is used to focus muons produced by neutrino interactions and measure their momentum.

An accurate determination of the absolute and relative energy response of the Near and Far Detectors is crucial to the success of the MINOS physics analysis. Since it was not possible to expose the Near and Far detectors to test beams, the MINOS collaboration constructed an additional Calibration Detector to characterize the calorimetric response of its detectors. The Calibration Detector was designed to be a "miniature" version of the Near and Far Detectors.

The MINOS detectors are described in further detail below:

- The Near Detector: The MINOS Near Detector (sce Figure 3.5) is located $100 \mathrm{~m}$ underground at Fermilab, $1 \mathrm{~km}$ downstream of the NuMI target. The beam has a diameter of approximately $50 \mathrm{~cm}$ when it reaches the Near Detector, and the detector is constructed to contain the neutrino interactions along the beam spot. The detector is composed of 282 planes of $2.54 \mathrm{~cm}$ steel each measuring $3.8 \mathrm{~m}$ by $4.8 \mathrm{~m}$. This gives it an overall mass of $0.98 \mathrm{kT}$. The detector is divided into four regions: an initial veto region used to veto neutrino interactions upstream of the detector; a target region which provides the fiducial volume used to select neutrino interactions for analysis; a shower region used to contain hadronic and electromagnetic showers produced by neutrino interactions in the target region; and a spectrometer region used to measure the muons produced by neutrino intcractions in the target region. In cach $8 \mu$ s beam spill, approximatcly 20 neutrino events are expected in the Near Detector. These multiple events overlap closely in space and time. The Near Detector uses a system of multi-ranging $Q I E$ readout electronics [75] to digitize detector hits into bins of $19 \mathrm{~ns}$ with no dead time. 
- The Far Detector: The MINOS Far Detector (see Figure 3.6) is located $700 \mathrm{~m}$ 1underground at Soudan, $730 \mathrm{~km}$ downstream of the NuMI target. The beam has a diameter of approximately $1 \mathrm{~km}$ when it reaches the Far Detector. This reduces the beam intensity by a factor of $\sim 10^{5}$ relative to the Near Detector. The Far Detector is therefore more massive than the Near Detector, consisting of 486 planes of $2.54 \mathrm{~cm}$ thick steel measuring $8 \mathrm{~m}$ by $8 \mathrm{~m}$. This gives the detector an overall mass of $5.4 \mathrm{kT}$. The expected neutrino interaction rate in the absence of neutrino oscillations is 1,600 per year for the LE beam.

- The Calibration Detector: The MINOS Calibration Detector was constructed to measure the response of the MINOS detectors and readout systems to test beams. During 2001-3, the detector was exposed to $0.2-10 \mathrm{GeV}$ beams of $p, \pi^{ \pm}$, $\mu^{ \pm}, e^{ \pm}$at the CERN PS accelerator in Geneva. The detector consisted of 60 planes of $2.5 \mathrm{~cm}$ thick stecl planes measuring $1 \mathrm{~m}$ by $1 \mathrm{~m}$. This gave the detector an overall mass of 12 tons. The detector was instrumented with readout components from both the Near and Far Detectors.

\subsection{Beam Neutrino Physics Analysis}

The MINOS experiment is capable of observing neutrino oscillations in a number of channels. The primary oscillation scarch is the measurement of $\nu_{\mu}$ disappearance in the NuMI beam due to $\nu_{\mu} \rightarrow \nu_{\tau}$ oscillations. MINOS will probe the region of parameter space favoured by the SKK experiment. In addition, MINOS offers a degree of sensitivity in the detection of $\nu_{e}$ appearance in the NuMI beam duc to sub-dominant $\nu_{\mu} \rightarrow \nu_{e}$ oscillations and will either observe these oscillations or tighten the existing limits.

In order to make precise measurements of oscillations, clean samples of $\nu_{\mu} \mathrm{CC}$ and $\nu_{e}$ $\mathrm{CC}$ events must be selected from the data. Since the beam is produced in spills, timing information is used to separate beam events from cosmic muons and detector noise. The $\nu_{\mu} \mathrm{CC}$ and $\nu_{e} \mathrm{CC}$ events must then be separated from NC events. The signature of a $\nu_{\mu}$ CC interaction is a muon track, with the main background arising from NC interactions which produce charged pion tracks. The signature of a $\nu_{e} \mathrm{CC}$ interaction is an electromagnetic shower, with the main background arising from NC interactions that produce neutral pion showers. Studies have shown that $\nu_{\mu} \mathrm{CC}$ [76] and $\nu_{e} \mathrm{CC}[77]$ events can be selected by analysing the event topology. 


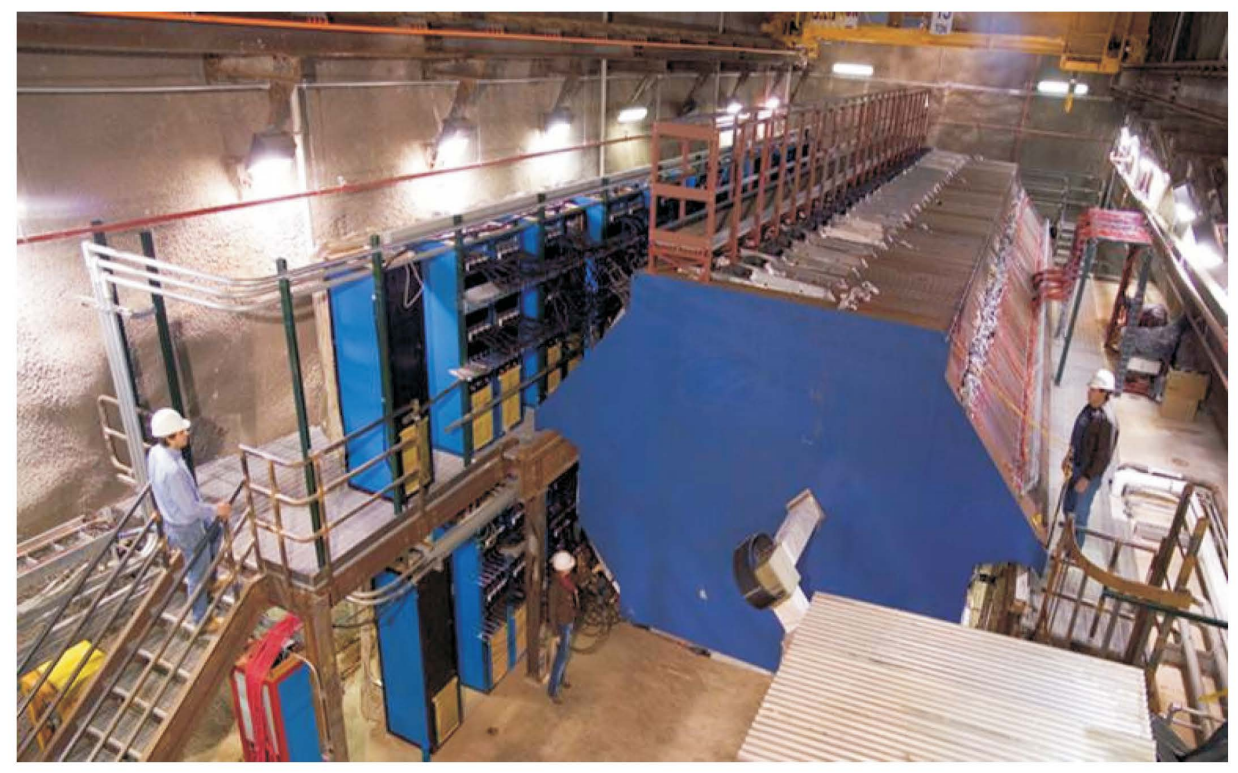

Figure 3.5: The MINOS Near Detector.

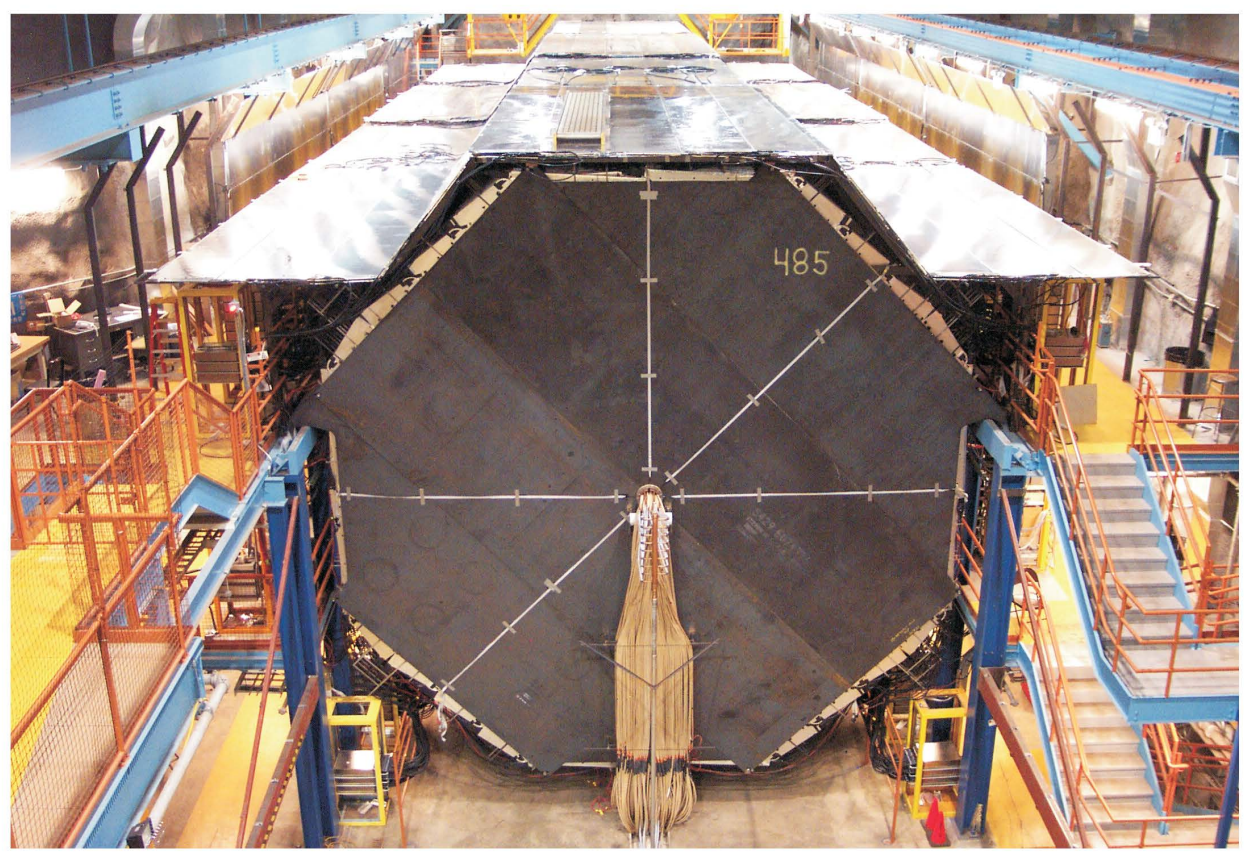

Figure 3.6: The MINOS Far Detector. 


\subsection{1 $\nu_{\mu} \rightarrow \nu_{\tau}$ disappearance search}

If $\nu_{\mu} \rightarrow \nu_{\tau}$ oscillations occur in the region of parameter space favoured by SKK, then MINOS will be capable of precisely measuring these oscillations. The NuMI beam parameters of $L=735 \mathrm{~km}$ and $E=1-5 \mathrm{GeV}$ provide sensitivity to oscillations in the region $\Delta m_{23}^{2} \geq 1 \times 10^{-3} \mathrm{eV}^{2}$. MINOS will search for $\nu_{\mu} \rightarrow \nu_{\tau}$ oscillations by comparing

the measured spectrum of $\nu_{\mu} \mathrm{CC}$ events at the Far Detector with a zero oscillation prediction obtained by extrapolating the measured spectrum of $\nu_{\mu}$ CC events at the Near Detector. The signature of neutrino oscillations is a periodic energy dependence in the Near-to-Far ratio of the measured Far Detector spectrum and the extrapolated Near Detector spectrum. The ratio dips at neutrino energies corresponding to high oscillation probabilitics. An observation of this periodic structure would represent definitive cvidence of neutrino oscillations. The value of the mass splitting $\Delta m_{23}^{2}$ can be determined by measuring the position of the dip in the Near-to-Far ratio. The size of the mixing angle $\theta_{23}$ can be determined by measuring the amplitude of the dip.

Figure 3.7 shows the expected sensitivity to $\nu_{\mu} \rightarrow \nu_{\tau}$ oscillations with the $L E$ beam for $25 \times 10^{20}$ protons on target (5 years running) [78]. The distributions of Near-toFar ratios and confidence limits for $\Delta m_{23}^{2}$ and $\sin ^{2} 2 \theta_{23}$ are shown for input oscillation parameters spanning the parameter space favoured by SKK at $90 \%$ confidence. In cach case a clear dip is observed in the Near-to-Far ratio at the oscillation maximum, enabling the parameters $\Delta m_{23}^{2}$ and $\sin ^{2} 2 \theta_{23}$ to be determined with better than $10 \%$ accuracy. MINOS is able to discrimminate clearly between neutrino oscillations and alternative models which do not produce a dip in the Near-to-Far ratio such as neutrino decay [53] [54] and neutrino decoherence [55] [56].

\subsection{2 $\nu_{\mu} \rightarrow \nu_{e}$ appearance search}

If the mixing angle $\theta_{13}$ is non-zero, then $\nu_{\mu} \rightarrow \nu_{e}$ oscillations will occur in the NuMI beam. The current oscillation experiments have placed an upper limit on the value of $\theta_{13}$. MINOS is capable of extending this sensitivity, and will either improve on the existing limit or observe $\nu_{\mu} \rightarrow \nu_{e}$ oscillations if the value of $\theta_{13}$ is high cnough.

The $\nu_{e}$ appearance signal is an excess in the observed number of $\nu_{e} \mathrm{CC}$ events above the background at energies corresponding to high probabilities of $\nu_{\mu} \rightarrow \nu_{\tau}$ oscillations. The background consists of the natural $\nu_{e}$ component in the beam due to the decays of 

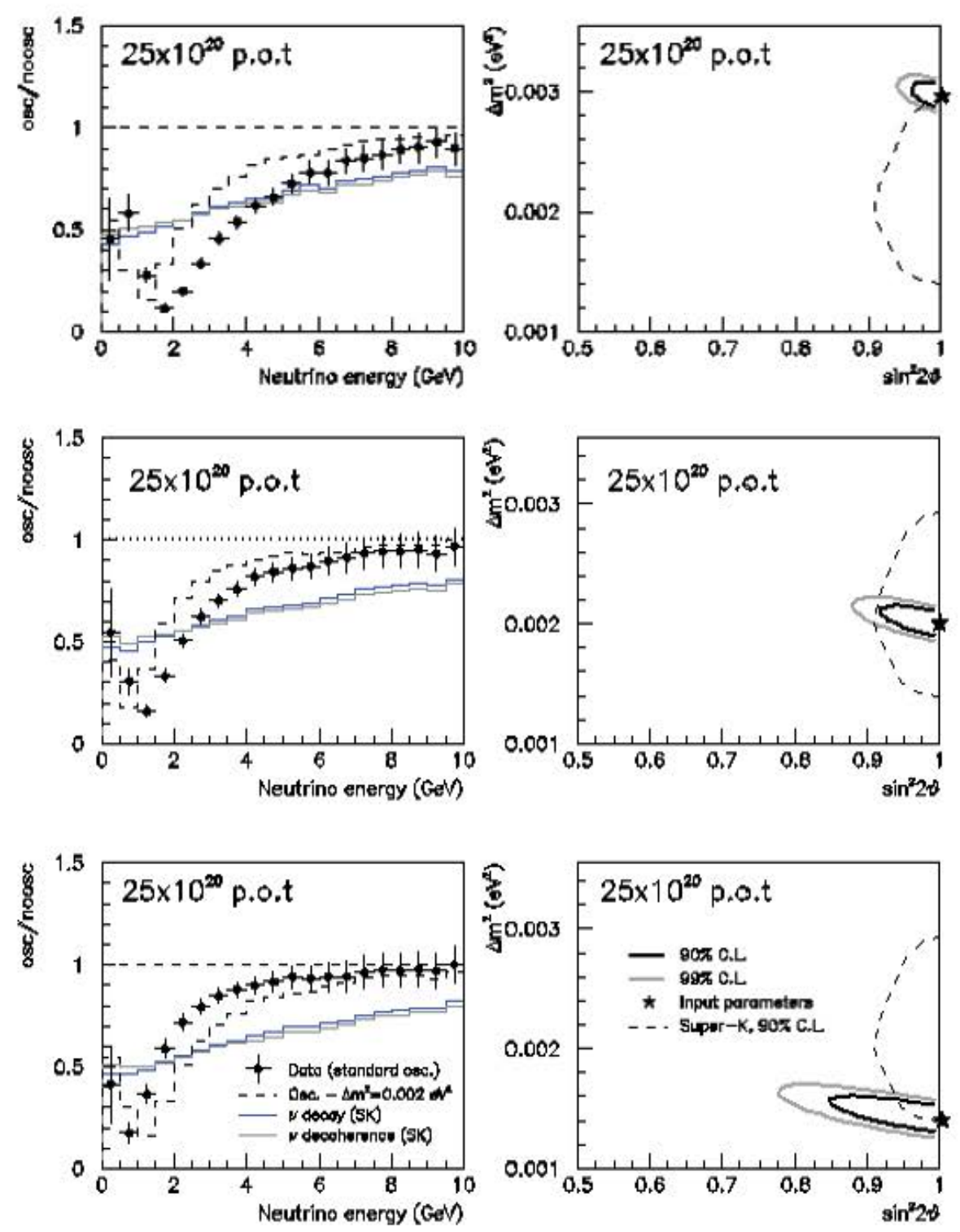

Figure 3. $i$ : MNOS senstimty to $\nu_{1 k} \rightarrow \nu_{-}$oscillation. The left punels show the expected Near-to-lar ratios for neutrino oscillation, neutrino decay and

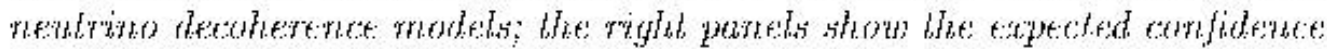

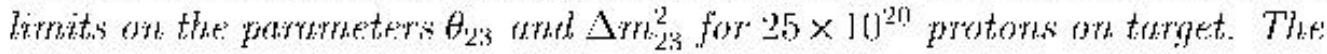

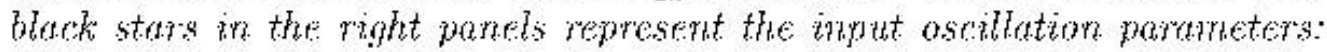
(Lop) $\Delta m_{23}^{2} \quad 2.95 \times 10^{-3} \mathrm{ct}^{2}, \sin ^{2} 20$ 1.0; middo; $\Delta m \frac{2}{2} \quad 2.0 \times$

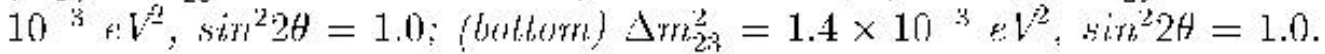
These purameters represtent the best fit values and go\% confidence limits for maximal mixing obtained by the shK experiment. 


\section{$3 \sigma$ Contours}

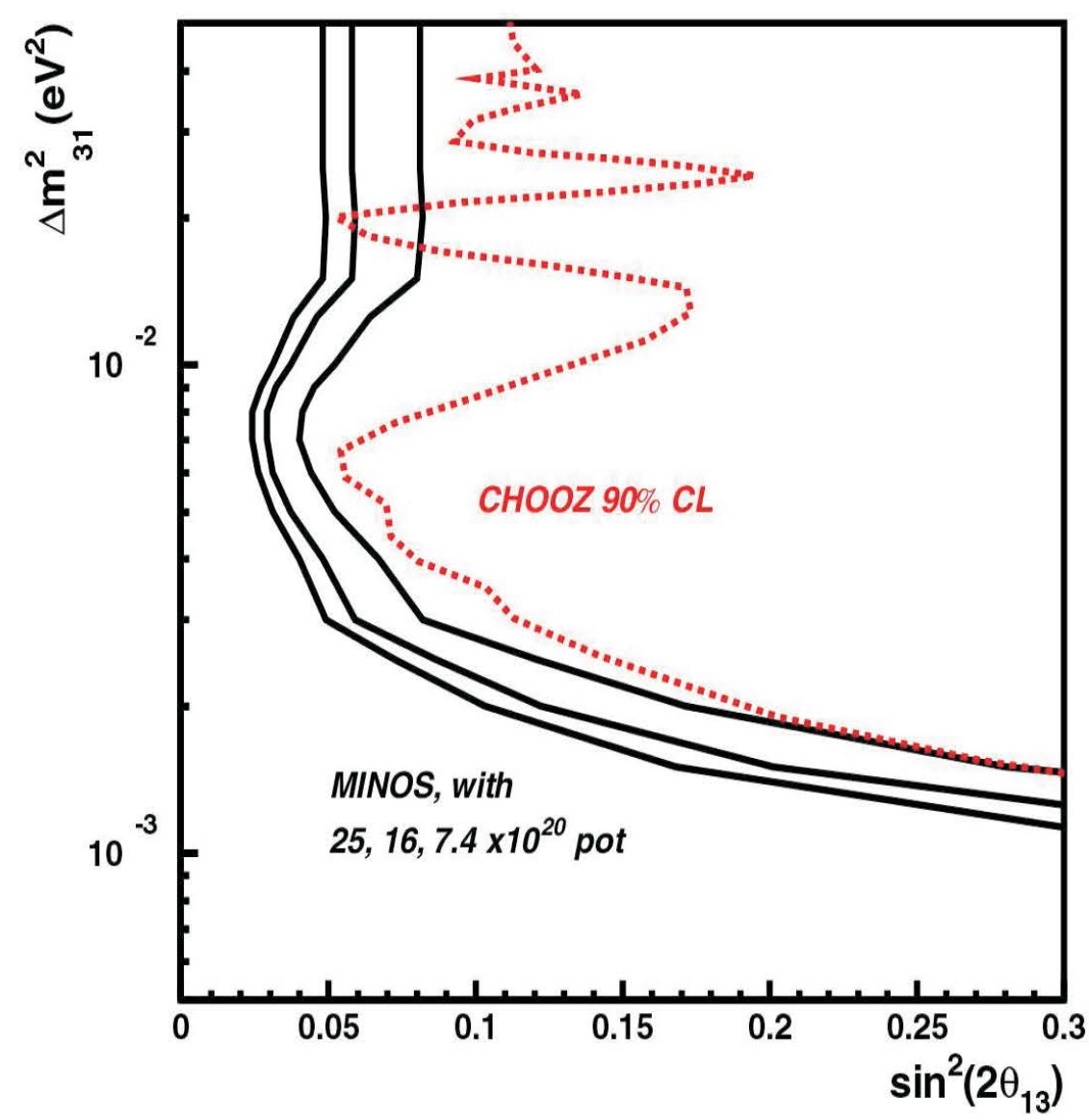

Figure 3.8: MINOS sensitivity to $\nu_{\mu} \rightarrow \nu_{e}$ oscillations. The solid lines represent the expected $3 \sigma$ confidence limits on the parameters $\theta_{13}$ and $\Delta m_{13}^{2}$ for 7.4, 26 and $25 \times 10^{20}$ protons on target. The dashed line represents the $90 \%$ limit obtained by the $\mathrm{CHOOZ}$ experiment.

$K^{+}$and $\mu^{+}$in the decay pipe. In addition, $\nu_{e} \mathrm{CC}$ events are mimicked by $\mathrm{\lambda C}$ interactions which produce neutral pions. The sensitivity of the $\nu_{e}$ appearance measurement is limited by crrors in the $\nu_{e}$ background expectation. These crrors can be reduced by measuring the $\nu_{e}$ component in the beam using the Near Detector, and by selecting $\nu_{e}$ CC events only at energies where $\nu_{\mu} \rightarrow \nu_{\tau}$ oscillations occur. Figure 3.8 shows the cxpected sensitivity to $\nu_{\mu} \rightarrow \nu_{e}$ oscillations with the $L E$ beam for 7.4, 16 and $25 \times 10^{20}$ protons on target (2, 4 and 5 years running) [78] compared with the CHOOZ limit $90 \%$ confidence limit. MINOS is sensitive to oscillations in the region $\theta_{13} \geq 5^{\circ}$. 


\subsection{Atmospheric Neutrino Physics Analysis}

The large mass and decp location of the MINOS Far Detector make it well suited for studies of atmospheric neutrino oscillations. In addition, the Far Detector is unique in being the first massive underground detector to possess a magnetic field, enabling separate measurements of neutrinos and anti-neutrino oscillations to be performed for the first time. This represents an extension on the physics capabilities of any other current or previous atmospheric neutrino experiment.

MINOS will study atmospheric neutrino oscillations by searching for distortions in the atmospheric $\nu_{\mu}$ flux relative as a function of $L / E$. The analysis requires the selection of a clean sample of atmospheric $\nu_{\mu}$ CC interactions. The atmospheric neutrino interactions must be separated from the large background of cosmic muons. Even at a depth of $700 \mathrm{~m}$ cosmic muons are incident on the Far Detector at a rate $\sim 10^{4}$ times greater than the rate of atmospheric neutrino interactions. Atmospheric neutrino events are identified by the following characteristic features:

- Contained Interaction Vertex: Atmospheric neutrinos interact inside the detector, whereas cosmic muons are incident on the edges of the detector. The interaction vertex of each event is measured using 3D reconstruction. Atmospheric neutrino events are sclected by requiring this vertex to be contained within the fiducial volume of the detector.

- Up-Going Muons: Events travelling upward through the detector originate from neutrino interaction. The direction of each event is measured by analysing timing information. Atmospheric neutrino events are selected by requiring the direction of the event to be up-going.

In order to perform an accurate measurement of oscillations in atmospheric neutrinos, a detailed understanding of the MINOS detector and data is required. The data must be well calibrated and the detector and incident particles well simulated. Atmospheric neutrino events must be separated cleanly from the cosmic muon background and the neutrino kinematics must be reconstructed accurately. This thesis presents a study of atmospheric neutrino oscillations in the MINOS Far Detector. 


\section{Chapter 4}

\section{The MINOS Far Detector}

\subsection{Detector Overview}

The MINOS Far Detector is located $700 \mathrm{~m}$ 11nderground at the Soudan Underground Laboratory in northern Minnesota, USA. The detector is a sampling calorimeter, composed of many interleaved planes of stecl and plastic scintillator. It is $30 \mathrm{~m}$ long, $8 \mathrm{~m}$ wide, $8 \mathrm{~m}$ high and weighs $5.4 \mathrm{kT}$. The detector was completed in August 2003 and began collecting cosmic ray and atmospheric neutrino data.

The detector is constructed from 486 octagonal stecl planes. The planes are $8 \mathrm{~m}$ wide and $2.54 \mathrm{~cm}$ thick, with their centres spaced apart by $5.94 \mathrm{~cm}$. The detector is divided into two sections, termed supermodules, containing 249 and 237 planes respectively. The stecl planes are backed with $1 \mathrm{~cm}$ thick layers of plastic scintillator, but cach supermodule begins and ends with steel so the two supermodules contain 248 and 236 scintillator planes respectively. The planes are aligned vertically to maximize sampling of bearn neutrino interactions.

The scintillator planes are divided into 192 parallel strips, $4.1 \mathrm{~cm}$ wide and up to $8 \mathrm{~m}$ long. Planes are aligned alternately along the orthogonal axes $U=\frac{1}{\sqrt{2}}(x+y)$ and $V=\frac{1}{\sqrt{2}}(-x+y)$. Each plane provides a high spatial resolution in either the $\mathrm{U}$ or $\mathrm{V}$ view, and information in the two views can be combined to give 3D measurements of events. The strips in each plane are housed inside 8 modules containing either 20 or 28 strips covered by a thin aluminium casing. Scintillation light generated in the strips by the passage of charged particles propagates in both directions along the strip. The light is collected by optical fibres and transported to photo-multiplier tubes. The signal is 
amplified by the photo-multiplier tubes and then digitized by sensitive electronics.

Each supcrmodule is magnetized using 190 turns of copper wire carrying a current of $80 \mathrm{~A}$ and running through the centre of the planes. This produces a toroidal magnetic ficld in the stecl with a mean strength of $1.3 \mathrm{~T}$. The ficld is designed to maximize the containment of charged particles and assist in the determination their momentum from the curvature of their tracks in the field. The direction of the field is chosen to focus negatively charged muons produced by beam neutrino interactions.

A veto shield of scintillator modules has been constructed above the detector to tag cosmic muons entering the detector. Since the flux of muons is peaked towards vertical angles, muons are mainly incident on the top face of the detector. However, the scintillator planes in the detector are aligned vertically and so provide $<20 \%$ coverage of the top surface of the detector. The shield modules are aligned along the $\mathrm{Z}$-axis and provide $>99 \%$ coverage of the top surface of the detector [72].

Figure 4.1 shows an end-on photograph of the Far Detector along with a schematic diagram illustrating the detector geometry, co-ordinate system and magnetic ficld direction. Figure 4.2 shows a steel plane instrumented with scintillator modules along with a diagram illustrating the arrangement of modules on each plane.

\subsection{Detector Technologies}

\subsubsection{Plastic Scintillator}

Physics events occurring inside the MINOS detector are sampled by the planes of active scintillator. The scintillator strips are manufactured from extruded polystyrene. Charged particles passing through the strips leave trails of excited molecules in their wake. The polystyrene contains aromatic molecules which, when excited, release a small fraction of energy as optical photons. The polystyrene is doped with primary (1\% PPO) and secondary $(0.03 \%$ POPOP) fluors which absorb the photons at a fast rate and reradiate them at a displaced wavelength where the scintillator is more transparent. In order to maximize the light yield, the strips are also lined with a reflective coating of polystyrene mixed with $15 \% \mathrm{TiO}_{2}$.

The scintillation light is collected by $1.2 \mathrm{~mm}$ diameter Kuraray Y-11 wavelength 

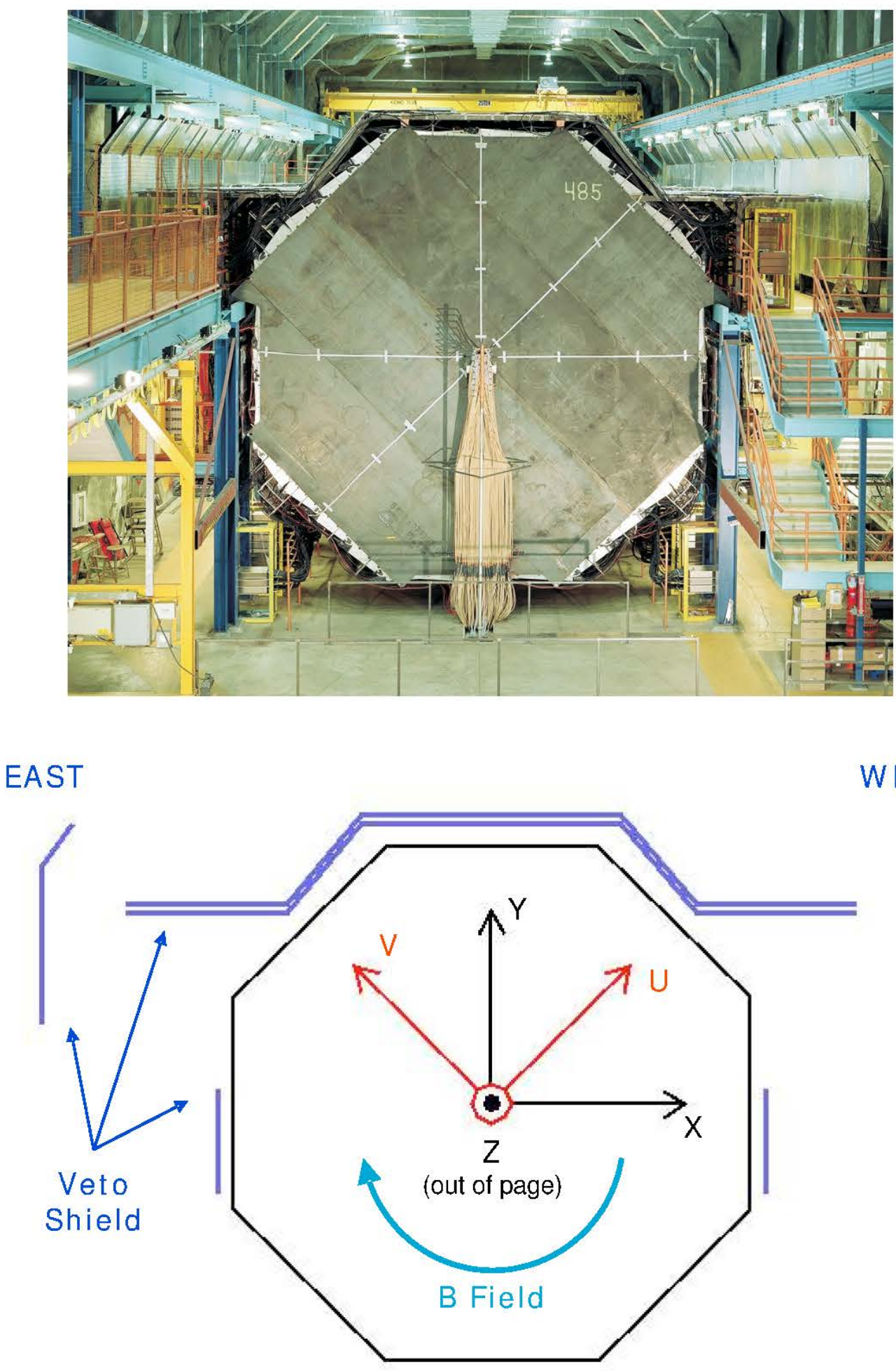

WEST

Figure 4.1: The MINOS Far Detector (viewed towards Fermilab). 

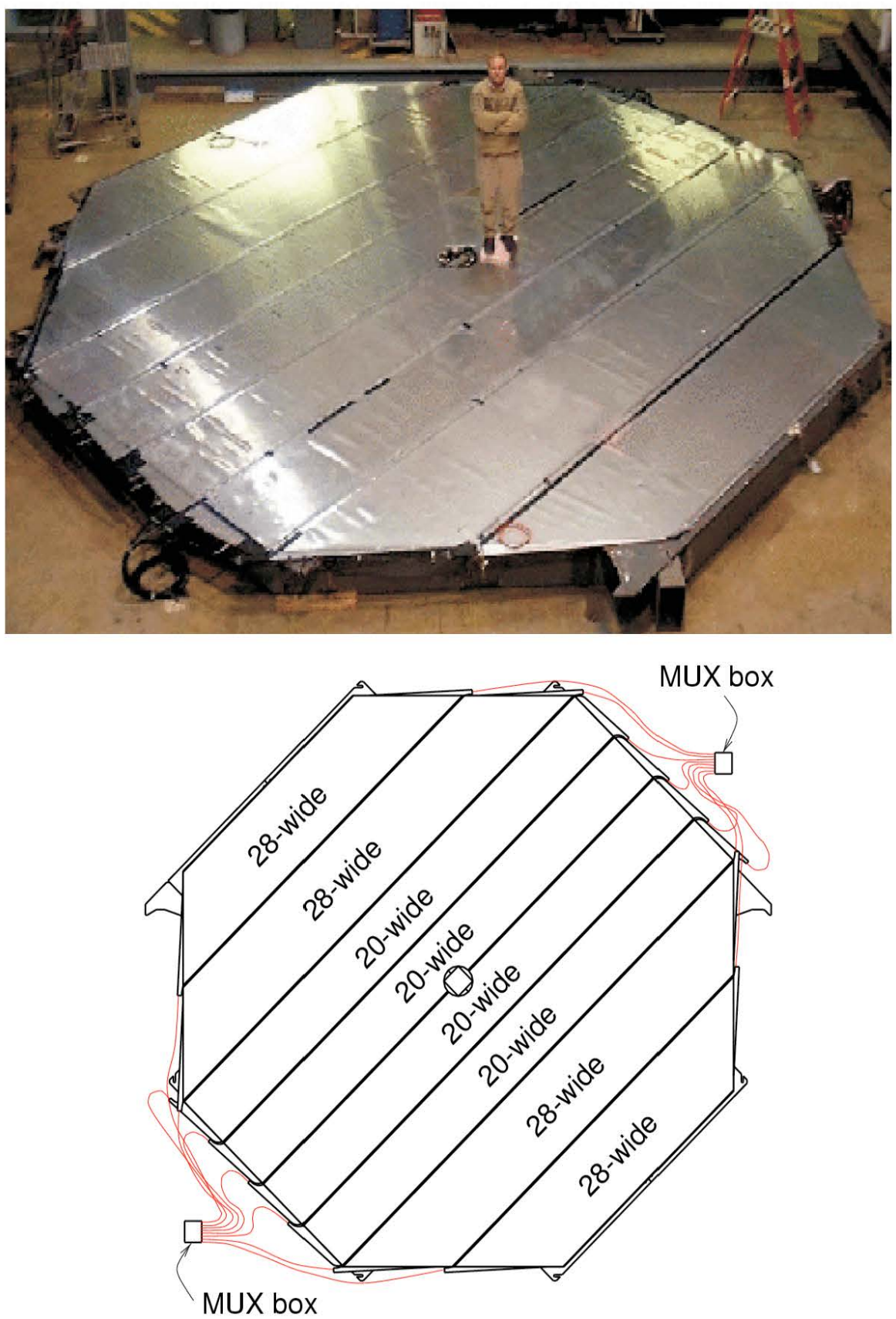

Figure 4.2: Far Detector scintillator plane. Each plane is composed of eight aluminium-clad modules containing either 20 or 28 scintillator strips. The signals at each strip end are transported along optical fibres from the scintillator to optical connectors at the edges of the modules. 
shifting (WLS) fibres glued into grooves cut along the wider edge of the strips and running along their entire length. The WLS fibres absorb light in the blue part of the spectrum, with peak absorption at $420 \mathrm{~nm}$. Light is then re-emitted in the green part of the spectrum, with peak emission at $520 \mathrm{~nm}$ [70]. The decay time for emission of light by the WLS fibres is approximately 8 ns [70]. The attenuation of light along the WLS fibres is described by two characteristic attenuation lengths. The strong absorption of light at short wavelengths is characterized by a short attenuation length; and the attenuation of the remaining longer wavelength light is characterized by a longer attenuation length. For the WLS fibre used at the Far Detector, these attenuation lengths have been measured to be $\lambda_{\text {short }} \simeq 1.0 \mathrm{~m}$ and $\lambda_{\text {long }} \simeq 7.0 \mathrm{~m}$ respectively [79] [80].

At each strip end, additional short lengths of WLS fibre are used to transport the light to the optical connectors located at each end of the scintillator modules. The light is then channelled along highly transparent clcar polystyrene fibres and transported to multi-pixel photo-multiplier tubes. The double-ended readout scheme ensures an approximately 11niform response along the strips since the signals at the ends of cach strip are summed to give the overall response of the strip. The overall response varies by up to $20 \%$ depending on the intial position along the strip.

\subsubsection{M16 Photo-multiplier Tubes}

The scintillator strips are read out by Hamamatsu M16 16-pixel photo-multiplier tubes (PMTs) [81]. Figure 4.3 shows a schematic of the Far Detector optical readout. Three PVTs on each side of the detector are used to read out a pair of scintillator planes in the either the $\mathrm{U}$ or $\mathrm{V}$ view. This gives a total of 1452 PMTs in the detector readout. Each group of three PMTs is housed in a custom-built light-tight multiplexing (MUX) box. Within each PMT, a group of fibres from 8 scintillator strips is coupled to each pixel. This creates an inherent eight-fold ambiguity in the readout scheme. However, the multiplexing of fibres into pixels on either side of the scintillator plane is designed such that each strip is read out by a unique pair of pixels. Therefore if the PMTs on opposite sides of a scintillator plane each record signals in only one pixel then a unique strip can be identified, and even if the PMTs record multiple hits it is usually possible to clearly define the event region.

Light signals generated in the scintillator are converted to photo-electrons at the PVT photo-cathodes. At the peak WLS fibre emission, the quantum efficiency for 


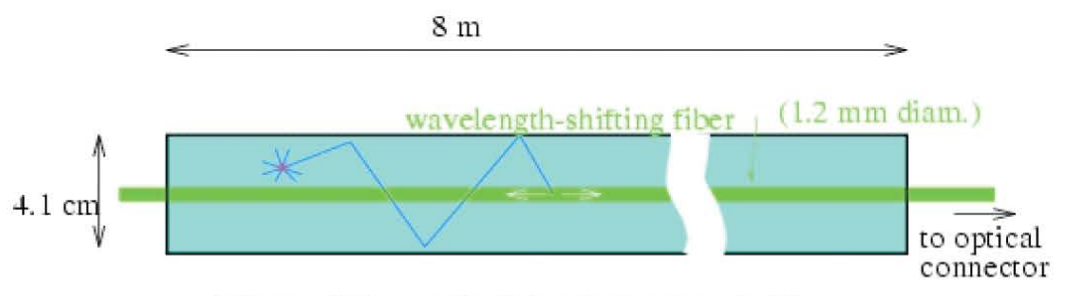

$1.0 \mathrm{~cm} \mathrm{x} 4.1 \mathrm{~cm}$ extruded polystyrene scintillator
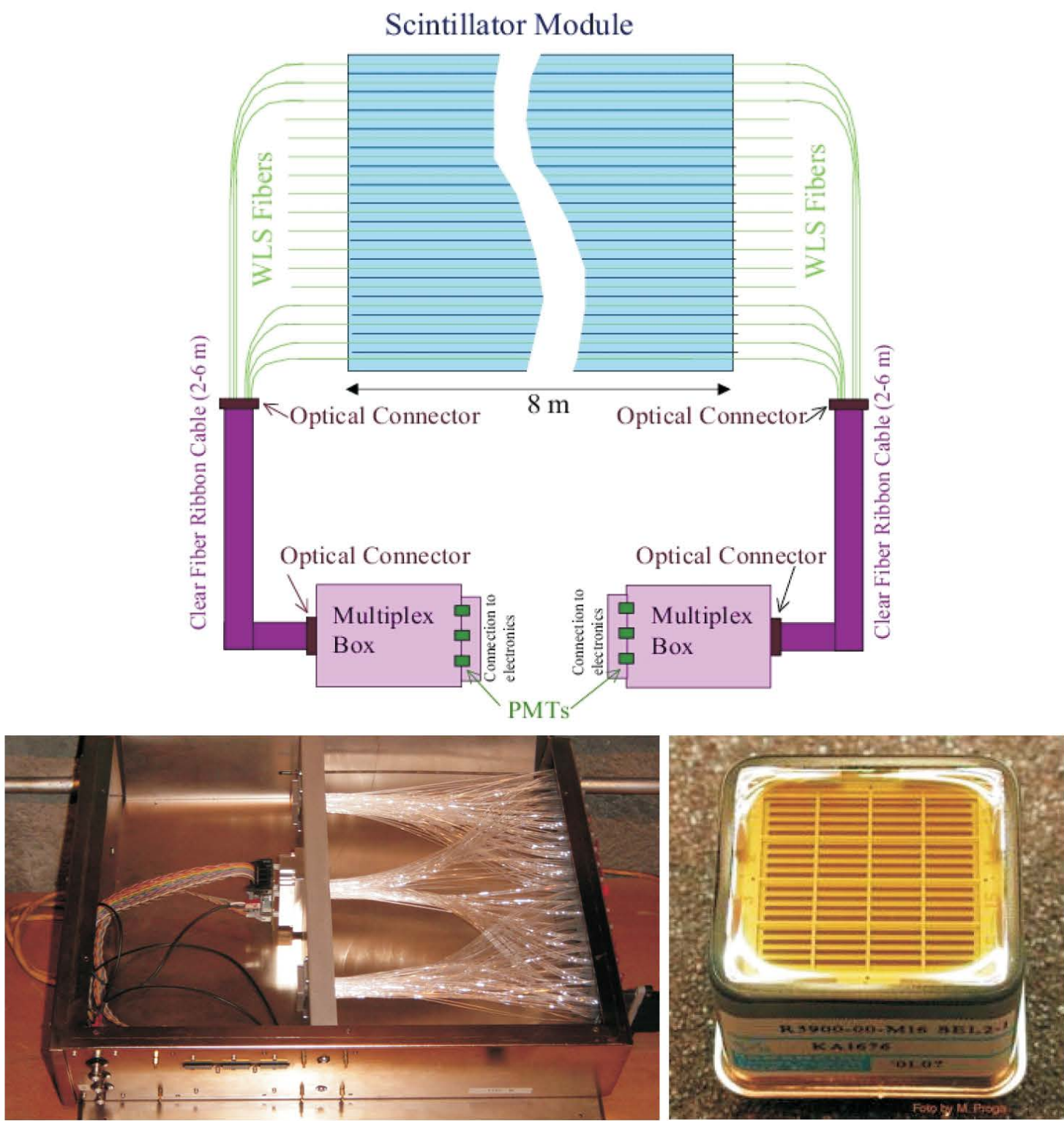

Figure 4.3: (Above) schematic of Far Detector optical readout. Light signals generated in scintillator strips are transported by optical fibres to M16 PMTs housed in custom-built MUX boxes. (Below) photographs showing optical fibres threaded into a MUX box (left) and an M16 PMT (right). 
photon conversion is approximately 13\%. A typical signal for a minimum ionizing muon is 5 PEs per strip end. The PMTs operate at gains of $10^{6}$ and typically produce signals of $1 \mathrm{pC}$ on the photo-anodes. Bench studies have shown that the integrated charge on the photo-anode is lincar with the input signal on the photo-cathode to within $5 \%$ for signals up to $100 \mathrm{PEs}[81]$.

Each P.T provides a common dynode signal which is used to trigger the readout. The trigger threshold is set below the single photo-clectron peak at a level of charge corresponding to $\frac{1}{3} \mathrm{PEs}$. However this is subject to variations in gain between pixels. The rate of dynode triggers caused by background noise in the detector has been measured to be $5-10 \mathrm{kHz}$ for each PMT. This is caused by the following effects [82]:

- Dark Photons: PMTs register dynode signals even when no input light is present. These arise chicfly from thermal cmissions of electrons from the photocathode which produce single photo-electron signals. The rate of these dark photons has been measured to be approximately $500 \mathrm{~Hz}$ per PMT.

- Natural Radioactivity: The detector components (stecl, scintillator, aluminium etc...) and surrounding rock contain small concentrations of long lived radioactive isotopes (e.g. ${ }^{238} U,{ }^{232} T h,{ }^{40} K$ ) whose complex decay chains produce a constant flux of $\beta$ and $\gamma$ radiation on the surface of the detector. One product of these decay chains is radioactive ${ }^{222} R n$ gas which is able to escape into the surrounding air and penetrate deep into the detector. The incident radiation produces energy deposits in the scintillator, resulting in signals of a few photo-clectrons.

Natural radioactivity at the Far Detector has been studied extensively. Based on these measurements, the predicted detector rates duc to radioactivity are $770 \mathrm{~Hz}$ per PMT, comprising: $410 \mathrm{~Hz}$ per PMT from natural radioactivity in the rock; $210 \mathrm{H} z$ per PMT from radioactivity in the detector; and $150 \mathrm{~Hz}$ per PMT from radon.

- Anomolous WLS Fibre Noise: Additional rates of approximately $5 \mathrm{kHz}$ per PMT are caused by photon emissions originating in the WLS fibre which result in single photo-clectron signals. The source of these photon emissions is belicved to be the long-term relaxation of mechanical stress caused by the glueing of WLS fibres into the scintillator strips during detector construction. 


\subsubsection{Front-End Electronics}

The P.MT photo-anodes are read out by a system of digital electronics. The Far Detector readout uses a customized version of the IDE AS Viking VA chip, an integrated circuit containing multiple channels of shaping amplifiers and sample-and-hold circuitry [83]. Each VA chip is responsible for reading out one PMT. The VA chips use 22 VA readout channels, with 1 channel allocated for each of the PMT photo-anodes; a further channel used to read out one of the PIN diodes that form part of the detector calibration system (sec Section 5.3); and the remaining 5 channels used to correct common mode fluctuations across the channels.

The Far Detector front-end readout scheme is illustrated in Figure 4.4. The VA clectronics is organized onto VA Front-End Boards (VFBs) located on the side of the MLX boxes, and VA Readout Control cards (VARCs) housed in VME crates some distance away. Three VA chips are housed on a VFB along with associated support circuitry. The readout of PMTs by VA chips is directed by Event Time Controller chips (ETCs) located on the VARCs. The VA chips read out each of the PMT photoanodes, amplify and shape the signals, and then send the signals to the VARC. The signals are digitized by Vare Mezzanine Modules (VMMs) which reside on the VARC. Each ETC-VMM pair services 2 VFBs, corresponding to 6 VA chips, and each VARC houses up to 6 ETC-VMM pairs. There are 3 VARCs in each VME crate, and 8 VME crates on each side of the detector.

The common dynode signals from each PMT are monitored using ASD-lite discriminator chips located on the VFBs. When a dynode signal is detected by the ASD-lite chip, a signal is sent to the ETC on the VARC. A coincidence trigger is implemented on the VARC which requires 2 out of 36 VA chips serviced by the VARC to trigger within 400 ns before the readout is activated. This reduces the trigger rate to $300 \mathrm{~Hz}$ per PMT. When an ETC receives a pair of dynode triggers that satisfy the 2/36 VARC trigger, it timestamps the triggers and directs the VA chips to read out the PMTs that have triggered. The signals in each of the VA channels are then digitized in turn by the VMM. During this time any further dynode signals from the PMTs are ignored. This means that a short dead time is incurred. The VA readout and digitization operates at $200 \mathrm{kHz}$ per chip, producing a dead time of $5 \mu s$ per VA chip. If multiple VA chips with a common VMM are triggered, they are queued by the ETC awaiting digitization. Since each VMM services 6 VA chips, dead times of up to $30 \mu s$ can occur. 
(a) VA Front-End Boards (VFBs)

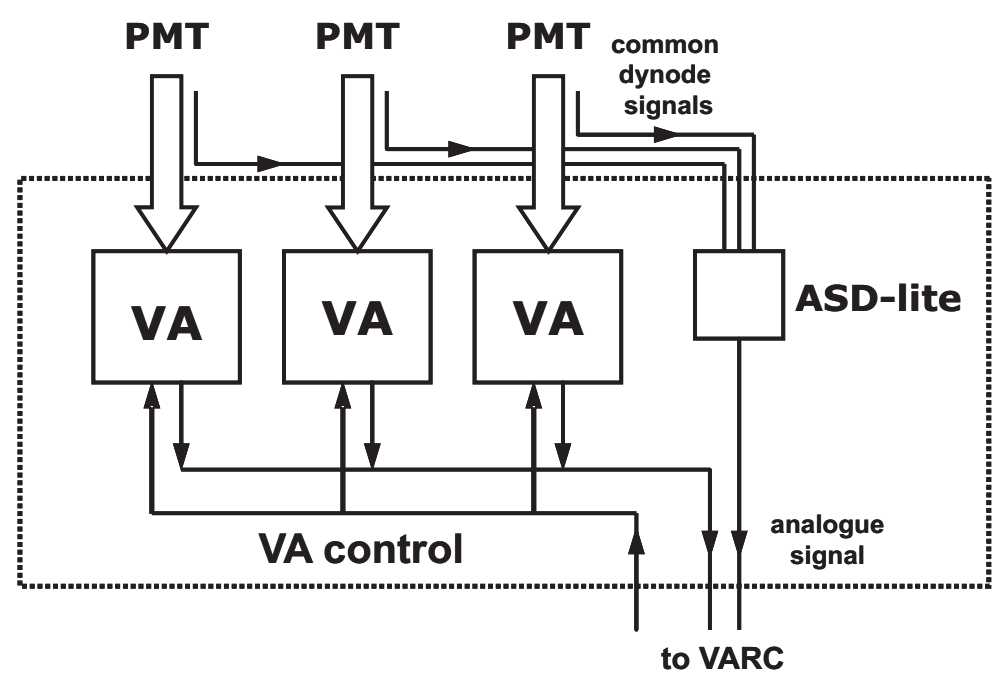

\section{(b) VA Readout Cards (VARCs)}
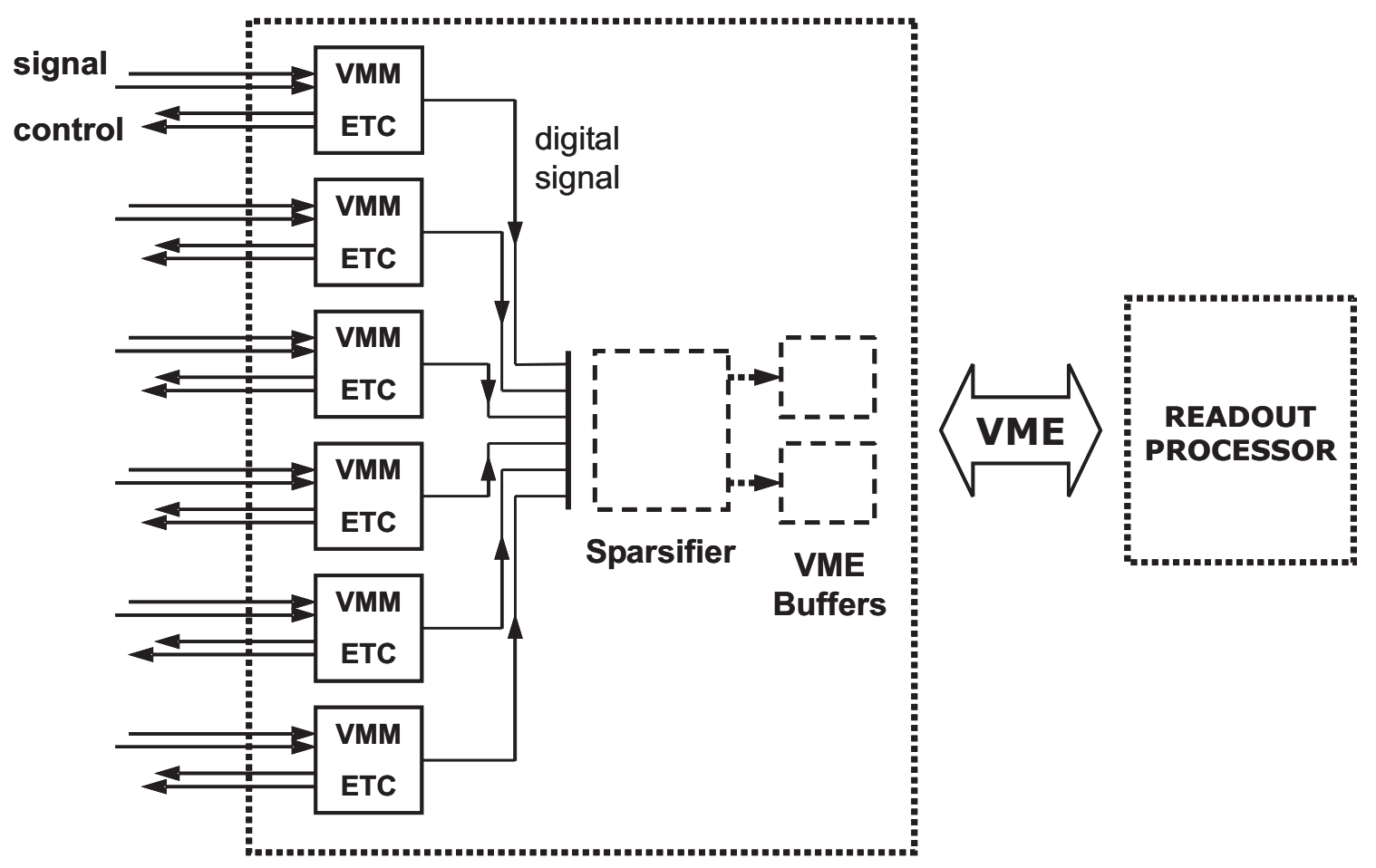

Figure 4.4: Far Detector front-end readout scheme. The VA electronics is organized onto VA Front-End Boards (VFBs) located on the side of MUX boxes, and VA Readout Control cards (VARCs) housed in VME crates. 
The VMMs digitize the VA signals into a 14-bit ADC. The dynamic range is set to approximately 70 ADCs per PE, giving a maximum signal of 300 PEs. The digitized signals are passed to a sparsifier chip on the VARC, which subtracts pedestal thresholds from each signal and sparsifies the data by removing all signals below 20 ADCs. The sparsified data is then transferred into one of two output buffers on the VARC. The two output buffers are used alternately to collect data from the VARC and transfer data out of the VARC, swapping roles at a rate of $50 \mathrm{~Hz}$. This cnables the data-taking to be continuous.

\subsubsection{Data Acquisition + Trigger Processing}

Each V.ME crate houses a single-board Readout Processor (ROP) which supervises the readout of data from the VARCs within the crate. The ROPs assemble the data into second-long time intervals known as timeframes. These timeframes are then passed through Branch Readout Processors (BRPs) to one of scveral Trigger Processors (TPs) running on a local farm of PCs. The task of the TPs is to search through the timeframes for events of physics interest.

The TPs time order the hits in each timeframe and divide them into groups separated from one another by at least 156 ns. These are then passed to a set of trigger algorithrns which search for spatial and temporal clusters in each group. If a group of hits satisfies one or more of these trigger conditions it is passed to the output stream of the TPs. The algorithm implemented at the Far Detector for cosmic ray and atmospheric neutrino data is a $4 / 5$ plane trigger algorithm which requires 4 out of 5 contiguous planes in the detector to register hits. Events written to the output stream of the TPs are collected by a Data Collection Process (DCP). These events are termed snarls. The events are written out and archived to the Fermilab mass storage system.

The Far Detector data acquisition (DAQ) [84] is co-ordinated by a central Run Control process [72]. Run Control directs the activity of all the DAQ processes, displays real time diagnostics of the state of the detector and the flow of data, and also provides a user interface for the DAQ. The collection of data is divided into many short segments called runs each lasting typically 2 hours. This enables data to be analysed offline in manageable segments. 


\subsubsection{Detector Control System}

To ensure safe and efficient detector operations, a Detector Control System (DCS) is used to control and monitor the state of the detector systems [85]. The DCS contains the following monitoring systerns:

- Environmental Controls: Sensors are placed around the cavern and on chosen detector planes to monitor the temperature and humidity inside the cavern and also the levels of radon gas.

- Low Voltage Monitoring: The VA chips are powered by $\pm 2.5 \mathrm{~V}$ voltage rails situated on the VFB. Voltage regulators on the VFB ensure quiet operation and the voltages are recorded by the VFB at the start of each run.

- High Voltage Monitoring: The PMTs are powered by eight high voltage mainframes. The typical voltage supplied to each PMT is $800 \mathrm{~V}$. The high voltage supply is controlled by custom control software, which reads the voltage on each PMT at regular intervals and attempts to adjust any voltages found to be out of range.

- Magnet Controls: Each magnet coil draws a 80 A current from a $450 \mathrm{~V}$ high voltage power supply. The power is ramped up and down using custom control software, and both the voltage and current are monitored at the supply. Thermocouples are also placed in several locations to monitor the coil temperature.

- Rack Protection Systems: Rack Protection systems (RPSs) are installed in each of the VME and DAQ racks around the detector. These systems monitor scnsors that are placed around the racks to measure environmental conditions and power supply voltages.

The DCS continuously collects monitoring data from each sub-system, providing detailed information on the operational state of the detector. This data is displayed and analysed in real time, allowing any problems to be identified and addressed. Most systems are also programmed to sound an alarm or automatically shut down if they are found to be operating outside normal conditions. 


\subsection{Data Quality}

All data used in the MINOS physics analysis must be checked to ensure that it has been acquired under normal conditions. The requirements for good data include:

1. All detector systems must be functioning normally.

2. There must be few malfunctioning readout components.

3. A large fraction of the detector readout must be live.

Several sources of detector and data monitoring are used to perform checks on the quality of physics data. The DCS provides data for all the detector systems. In addition, the DAQ monitors the quality of raw data and records any crror flags that have been registered by the VA electronics. The DAQ monitoring records include: trigger rates for cach PMT; measurements of temperatures and low voltage rails for cach VFB; and any buffer overflow flags that have been set by each VARC. The DCS and DAQ monitoring data is analysed to identify periods of abnormal detector running. This is then combined with information obtained from written logs and daily checks of physicists and enginecrs working on site at the Far Detector.

The data analysed in this thesis was acquired during the period 1st August 2003 to 31st October 2004. Data quality checks have been performed on all physics data acquired during this period. The main checks are discussed below.

\subsubsection{Coil Currents}

The magnetic ficld is essential to the MINOS physics analysis as it enables the charge sign and momentum of charged particles to be determined from the curvature of their trajectories through the detector. The DCS monitors the coils continuously and records the size of the coil currents at two minute intervals. Figure 4.5 shows the coil currents recorded in each supermodule for the period 1st August 2003 to 31st October 2004. During normal operations, the coil current remains stable at $80 \mathrm{~A}$. However, in $3 \%$ of physics data, the current is found to drop below $70 \mathrm{~A}$ in one or both of the supcrmodules due to power trips or hardware malfunctions. These data are removed from the physics analysis. On 21st June 2004 the polarity of the coil was reversed in order to study systematic effects in cosmic muon charge determination. 


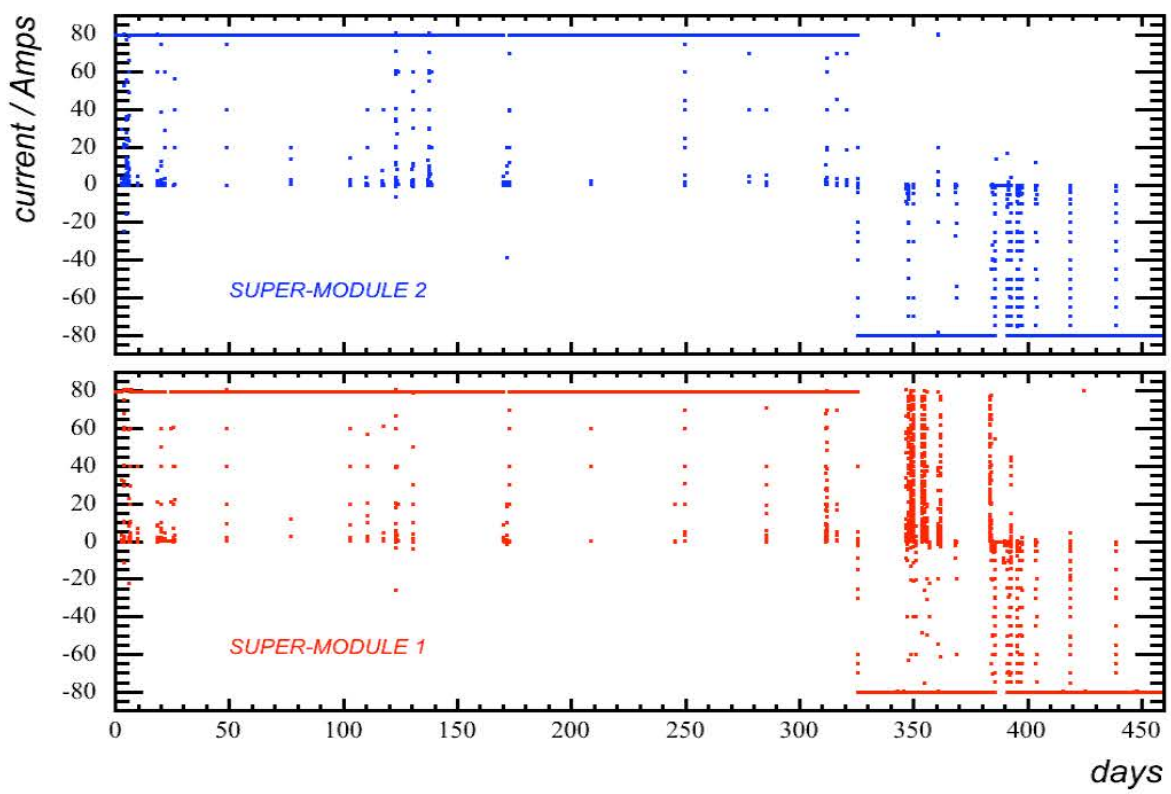

Figure 4.5: Coil currents versus days since 1st August 2003 (day 0). During normal operations, the coil draws a current of $80 \mathrm{~A}$. The polarity of both coils was reversed on 21 st June 2004 (day 325).

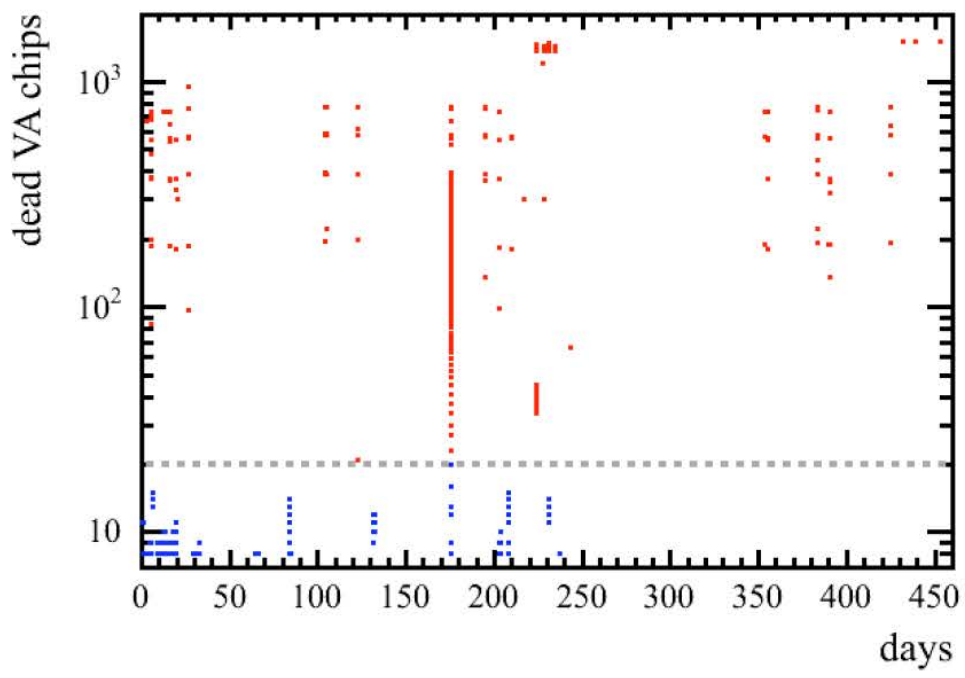

Figure 4.6: The number of dead VA chips versus days since 1st August 2003 (day 0). High voltage trips are flagged for $>20$ dead VA chips. 


\subsubsection{Dead Electronics}

Malfunctions in hardware can lead to localised regions in which the readout is dead. These readout holes are chicfly caused by malfunctioning readout clectronics and mainly affect single PVTs or VA chips dispersed through the detector. However, much larger dead regions are created by trips in one or more of the eight mainframe HV supplies that power the PMTs.

Readout holes in the detector can be identified using DAQ monitoring information. For each timeframe, the TPs record the rate of hits on each VA chip. A VA chip is flagged as dead if the rate drops below $50 \mathrm{~Hz}$. Figure 4.6 shows the number of dead VA chips plotted against time for physics data collected between 1st August 2003 and 31st Octobcr 2004. During normal detector operations this number remains below 10 VA chips. However, occasional spikes occur due to HV trips, which are flagged if the number of dead VA chips rises above 20.

Figure 4.7(a) shows the distribution of dead VA chips in data where the HV was found to be functioning normally. The mean number of dead VA chips is calculated to be 1.82 . This corresponds to $0.13 \%$ of the detector readout. Figure 4.7 (b) shows the mean number of dead VA chips plotted against time. This shows a clcar improvement over time, as the detector is better understood and bad components are replaced. By 1st October 2004 the number of readout holes has fallen to zero.

\subsubsection{Busy Electronics}

Each time a VA chip reads out a PMT, a period of $5 \mu$ s dead time is incurred, effectively creating a readout hole in the detector. The readout is activated when two dynode triggers are recorded that satisfy the 2/36 VARC trigger conditions. If the triggers occur in VA chips serviced by different VMMs ( $85 \%$ of the time), both VA chips will go dead for $5 \mu \mathrm{s}$. However if the triggers occur in VA chips serviced by the same VMM ( $15 \%$ of the time), one VA chip will be queued while the other is directed to read out, producing dead times of $5 \mu$ s and $10 \mu$ s respectively.

The mean rate of hits on each VA chip is $300 \mathrm{~Hz}$. This produces a dead time of $0.2 \%$ per VA chip corresponding to approximately 3 busy VA chips in the detector at any particular time. In order to identify which VA chips are busy at the time of an event, the TPs record the previous $30 \mu$ s of detector hits (the pre-trigger window) along with 

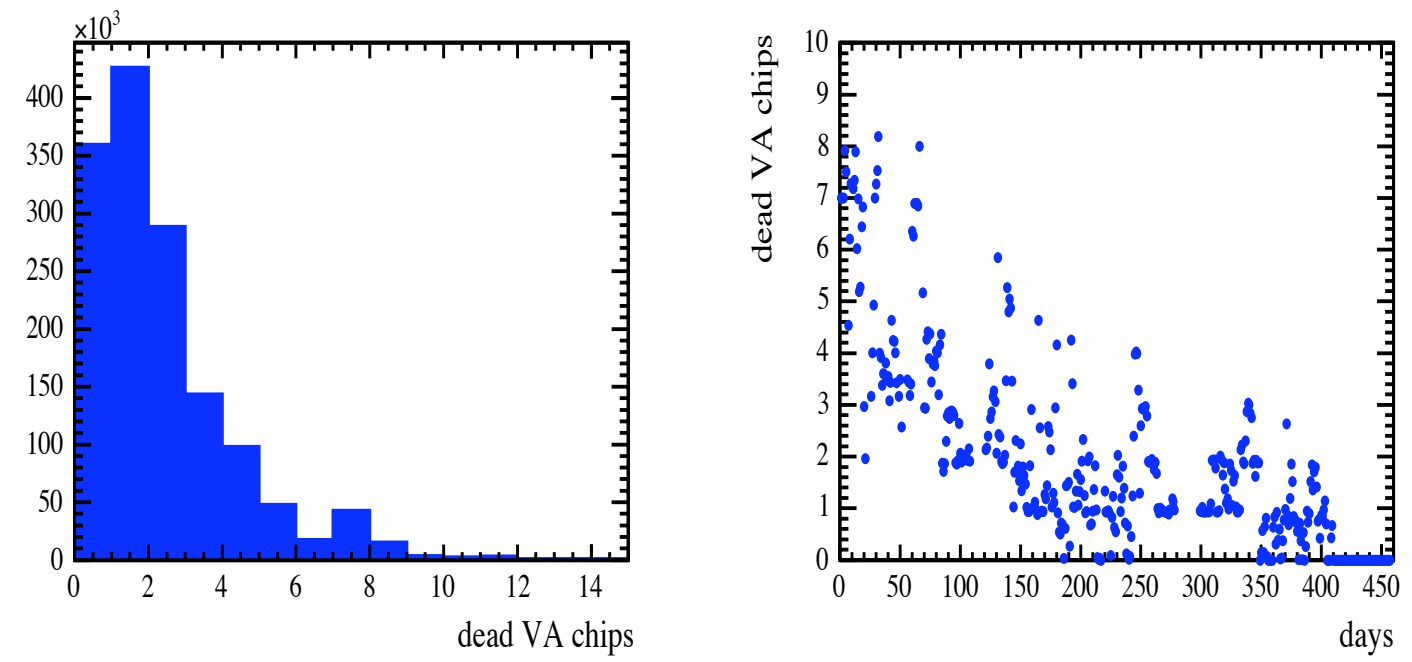

Figure 4.7: (a) Distribution of dead VA chips, using data where no HV trip has been flagged. (b) Mean number of dead VA chips versus time.
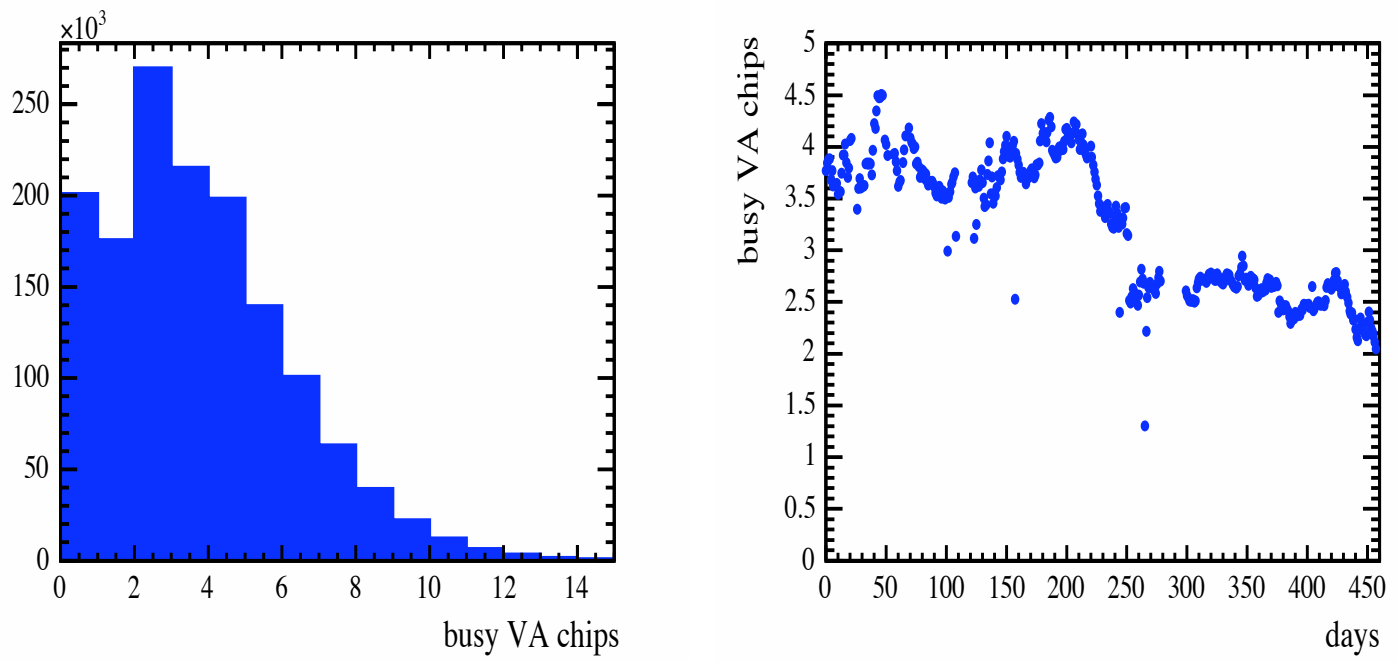

Figure 4.8: (a) Distribution of busy VA chips, obtained by analysing pretrigger windows. (b) Mean number of busy VA chips versus time. 
the hits associated with the event. The pre-trigger hits are then analysed offline to determine which VA chips were absent from the readout during the event. The $30 \mu \mathrm{s}$ time window corresponds to the maximum possible dead time for a VA chip.

Figure 4.8(a) shows the distribution of busy VA chips obtained using the pre-trigger windows of events. The peak at 2 arises from the VARC trigger conditions which require at least 2 coincident dynode triggers. Events with 1 busy VA chip occur when one of the dynode triggers is sparsified away. The vast majority of events contain less than 10 busy VA chips, with the mean number of busy VA chips calculated to be 3.29 , corresponding to $0.23 \%$ of the detector readout. Figure 4.8 (b) shows the mean number of busy VA chips plotted against time. This number slowly decreases over time, reflecting a reduction in detector noise over time. The drop at around 200 days occurred following a tuning of the P.MT dynode trigger thresholds.

\subsection{The Veto Shield}

A veto shicld has been constructed above the detector to cnable the selection of atmospheric neutrino events from the data [86]. Atmospheric neutrino events must be selected from a high background of cosmic muons. The veto shield is used to tag cosmic muons entering throwgh the top or sides of the detector. If a high tagging efficiency can be achieved then the cosmic muon background in the atmospheric neutrino event selection can be reduced to a low level.

The veto shicld is constructed out of the same scintillator modules as the scintillator planes in the detector. The modules are placed along the top and sides of the detector and are supported by a metal framework as well as by the detector itself. The shicld is divided into four overlapping sections aligned along the Z-axis. Sections 1 and 2 are located above the first supermodule; sections 3 and 4 are located above the second supermodule. Each section consists of a central double layer of modules directly above the detector, and two single layers diagonally above and directly to the side of the detector. This layout is illustrated in Figure 4.1. A double layer is used above the detector because this surface has the highest incident flux of cosmic muons and therefore requires the highest muon tagging efficiency. However, the majority of cosmic muons pass through two layers of scintillator before entering the detector. Figure 4.9 shows an example of a cosmic muon event tagged by the veto shield. 
The readout of the shield modules is integrated into the existing detector readout. Fibres run from both ends of the scintillator strips into M16 PMTs housed in MUX boxes. The multiplexing of fibres from the shield modules is identical on both sides of the detector, with eight adjacent strips coupled to each PMT pixcl. Each group of cight scintillator strips coupled to a single PMT pixel is known as a shield plank. Each plank has a spatial resolution of $10 \mathrm{~cm}$ in the X-Y plane. The PMTs are read out by VA chips and digitized by VMMs which share common VARCs with the detector readout. The trigger conditions are set differently for the shield readout in order to optimize the muon tagging efficiency. To reduce tagging inefficiencies that arise from single PE noise, the dynode threshold is set to a level equivalent to $1-2$ PEs. The shicld readout is also not included in the VARC trigger so that all shield activity is recorded.

A small fraction of cosmic muons are not tagged by the shield as they enter the detector. These shicld incfficicncies occur for a number of reasons:

1. A shield readout channel is dead due to noise or radioactivity.

2. A cosmic muon passes through the gap between two shield modules.

3. A shield activity is below the $1-2 \mathrm{PE}$ dynode threshold.

The tagging efficiency of each shicld plank is detcrmined 11sing a sample of cosmic muons which span $>20$ planes and have straight trajectories, with RMS deviations of $<0.5 \mathrm{~cm}$ from linear fits in both the $\mathrm{U}$ and $\mathrm{V}$ views. The muon trajectories are projected lincarly back to the shicld to identify which planks they have passed through. The projected trajectories are required to pass $<5 \mathrm{~cm}$ from the centre of planks to ensure that the correct planks have been identified. The shield efficiency is then determined for each plank by calculating the percentage of muon tracks tagged by shicld hits within a window of \pm 100 ns around the projected muon time at the shield. Figure 4.10 shows the tagging efficiencies of each plank in cach shicld section. The vast majority of planks have efficiencies of $>90 \%$, with the mean efficiency calculated to be $94.9 \%$. Therefore, the shield can be used to reduce the cosmic muon background by a factor of $\sim 20$. 


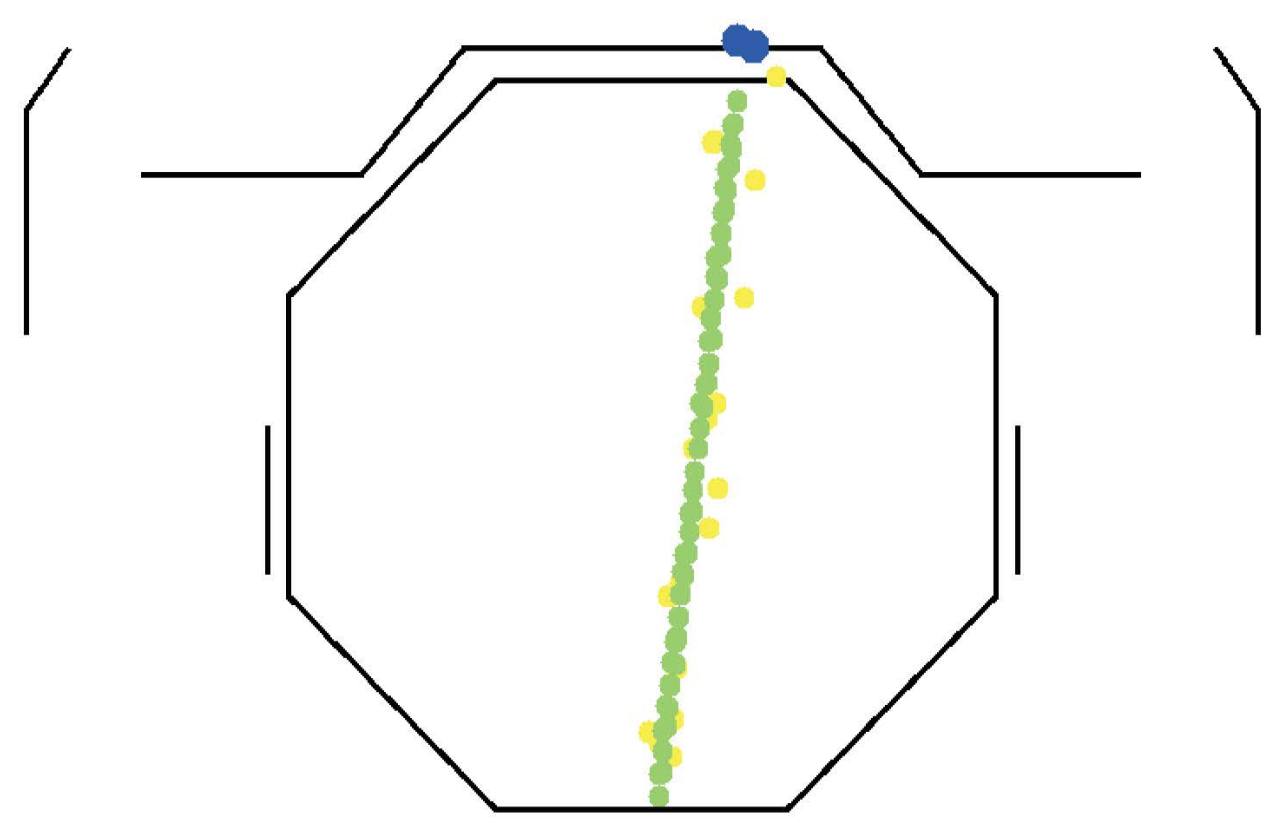

Figure 4.9: Example of a cosmic muon tagged by the veto shield. The event has been de-multiplexed to give the positions of the hits in the $U-Z$ and $V-Z$ views. These are then combined to give $3 D$ hits in the $X-Y$ view. The yellow points represent hits with $<2$ PEs; the green points represent hits with $>2$ PEs; the large blue points indicate activity in the veto shield. 

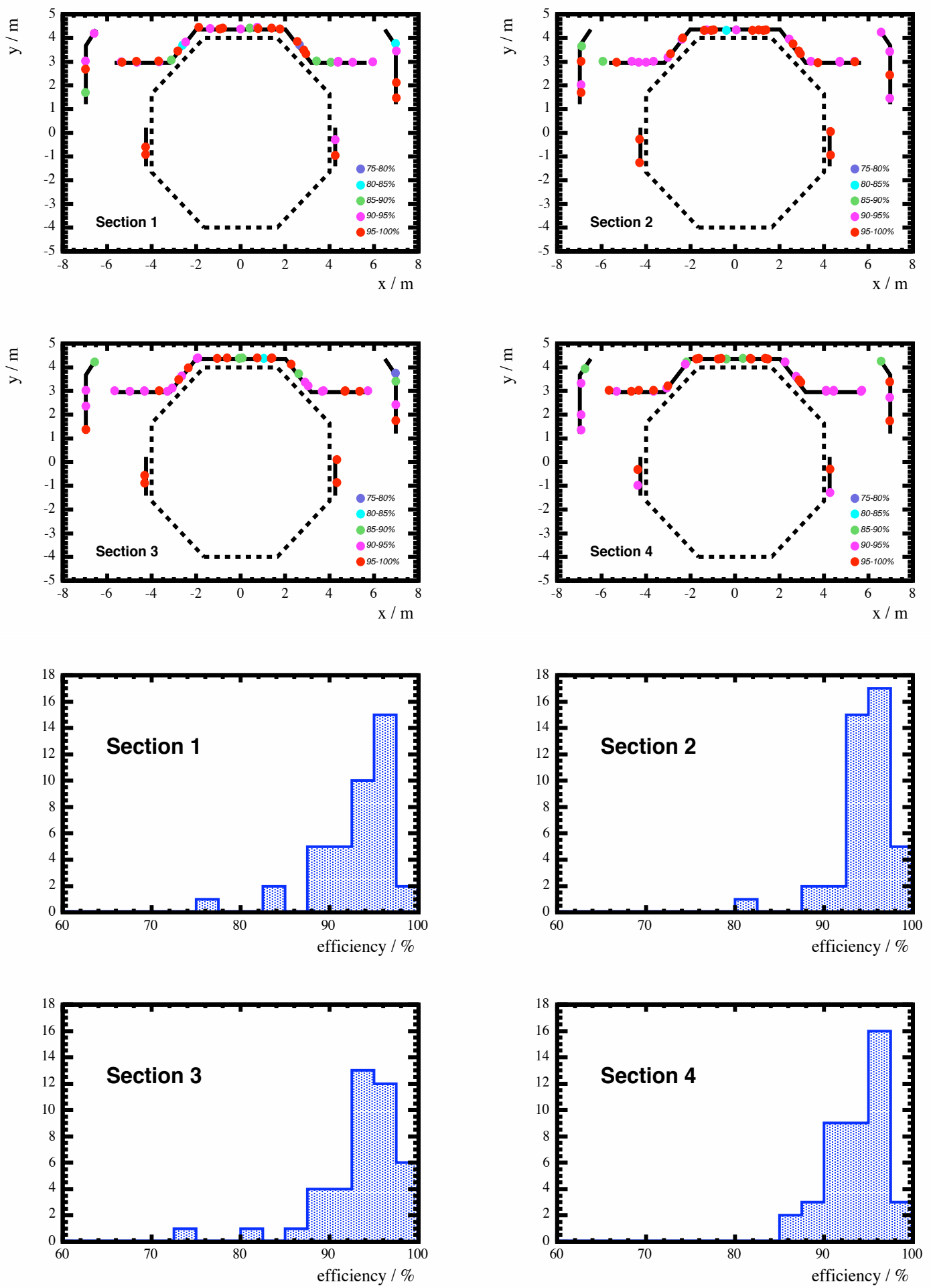

Figure 4.10: (a) cosmic muon tagging efficiencies for each shield section. (b) distribution of muon tagging efficiencies for each shield section. 


\section{Chapter 5}

\section{Detector Calibration}

\subsection{Calibration Overview}

The Far Detector must be calibrated to correct for variations in the response of scintillator strips and readout channcls in the detector. For cach physics event, the detector records a series of hits which sample the event and provide measurements of timing and pulse height. These measurements are used to reconstruct the event. The timing and pulse height information is digitized into finc-grained bins to maximize the resolution of the detector. However, large constant offsets in time and variations in response exist across the detector which must be corrected. A calibration scheme is implemented at the Far Detector to characterize cach scintillator strip and readout channcl by measuring of their response to well understood or common sources.

The calibration of the timing system is carried out 11sing cosmic muon data. High energy cosmic muons pass through the detector at a velocity close to the speed of light. Time offsets between scintillator strips and readout channels are measured by comparing the relative time and separation of detector hits along cosmic muon tracks.

The linearity and drift in the gain of each readout channel is measured using two injection systems. A system of Light Injection (LI) is used to inject controlled quantities of light into the scintillator strips; and a system of Charge Injection (CI) is used to inject controlled quantities of charge into the readout electronics. The relative response of each scintillator strip and readout channel is then determined using cosmic muon data assuming a common profile of energy deposition across the detector. 


\section{$5.2 \quad$ Timing Calibration}

Timing information is used to determine the propagation direction of physics events in the Far Detector. In order to maximize the physics sensitivity of the atmospheric neutrino analysis, the up-going and down-going events must be cleanly scparated. Events reconstructed as up-going provide one of the characteristic signatures of neutrino interactions. Any event travelling up through the detector must originate from a neutrino interaction. However, these up-going neutrino-induced events must be selected from a very high background of down-going cosmic muons. This means that the event direction must be reconstructed very precisely from the timing information.

The Far Detector is capable of performing timing measurements at the level of nanoseconds. The detector hits are digitally timestamped by the readout electronics into bins of $1.5625 \mathrm{~ns}$. This gives an intrinsic rms resolution of $0 . \overline{\mathrm{ns}}$. However, in practice the timing resolution is controlled by the 8 ns decay time of the WLS fibre. When this decay time is combined with the average signal generated on the P.TTs by the passage of minimum ionizing particles through the detector, a timing resolution of $\sim 2 \mathrm{~ns}$ is obtained. In comparison to this resolution, a $1 \mathrm{GeV}$ muon takes 10 ns to come to rest in the detector. This suggests that the detector should be very sensitive to the direction of physics events. However, a series of constant time offsets and delays exist within the timing system that are large compared to the resolution. These must be measured and corrected in order to optimize the resolution. Timing corrections must be applied to account for the following effects:

- Electronic Offsets: The Far Detector timing system is stable to $<1$ ns over long periods of time, but is synchronized across the detector to $<30 \mathrm{~ns}$. The system is controlled by a Timing Central Unit (TCU), which is clocked by a GPS system. This unit fans out control signals via optical cables to Timing Receiver Cards (TRCs) housed in each of the VME crates. These TRCs in turn generate signals in the VARCs. The short time delays associated with sending these signals mean that constant timing offsets exist between the timestamps recorded by the detector readout components. The primary timing offsets in the detector readout are between the VARCs, but there are also small but significant offsets between the PMTs and VFBs read out by each VARC, due to variations in the rise time of dynode trigger signals. 
- Hardware Changes: Shifts in the timing calibration of around 5 ns are caused by changes of hardware components in the detector readout system: each new PMT possesses a different characteristic signal rise time; each new VFB is fitted with a new dynode trigger chip; and each new VARC reccives timing signals from the TRCs with slightly modified arrival times. Changes in detector hardware occur on a weekly basis, and the resulting shifts in the timing system must be corrected before any long term timing calibration can be carried out.

- Strip-to-Strip Calibration: Additional small timing offsets exist upstream of the readout electronics, created by effects such as variations in the lengths of optical fibres read out by each P.MT and variations in rise times of the dynode signals within a PMT. A full strip-to-strip timing calibration must be performed to correct for these effects.

- Time Walk: The number of photons yielded at each strip end by the passage of minimum ionizing particles through the scintillator is low, typically 40 photons. The trigger time is determined by the arrival time of the first photon that converts on the photo-cathode, and by the rise time of the dynode signal through the PMT. For large photon yields, the trigger time is likely to be prompt; for low photon yields, the trigger time is skewed towards later times. This time delay is known as time walk, and can be as high as $10 \mathrm{~ns}$ for low signals.

A timing calibration of the Far Detector has been carried out using data acquired between 1st August 2003 and 31st October 2004. The timing calibration must be accurate to $<1$ ns in order not to degrade the overall resolution of the timing system. There must also be few poorly calibrated channels, since atmospheric neutrino events are typically short and their propagation direction must be detcrmined from a relatively small number of hits. The timing calibration is performed using through-going cosmic muons selected from the data. These high energy muons travel close to the velocity of light. This cnables timing fits to be applied to the measured times and positions along the muon tracks constraining the muon velocity. The timing fits are used to calculate the average time offsets associated with each of the above effects. The timing calibration procedure is described in detail below. 


\section{Time Calibration Procedure}

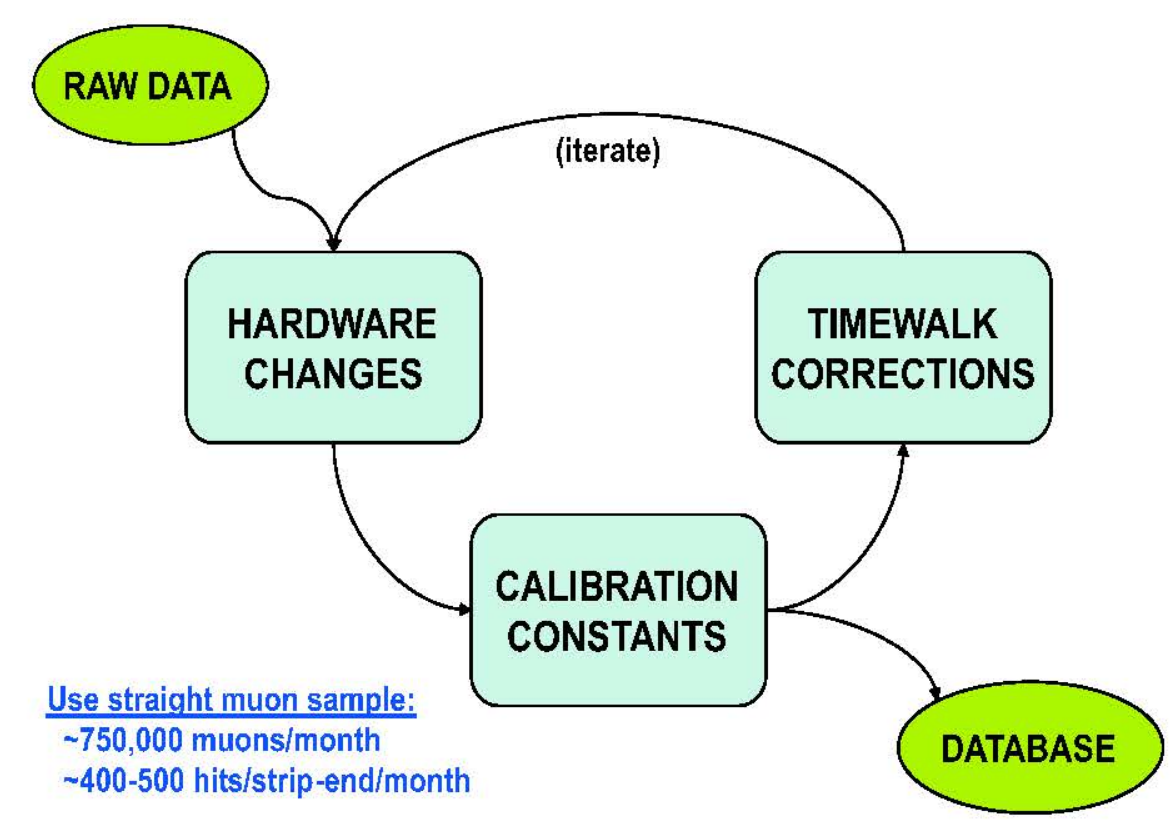

Figurc 5.1: The timing calibration procedure.

\subsubsection{Calibration Procedure}

The overall time correction $\Delta \mathrm{T}$ applied to each scintillator strip is given by the sum of the following different types of correction:

$$
\Delta \mathrm{T}=\Delta \mathrm{T}_{0}+\Delta \mathrm{T}_{j u m p}\left(t>t_{\text {jump }}\right)+\Delta \mathrm{T}_{\text {walk }}(Q)
$$

where:

$\Delta \mathrm{T}_{0}=$ combincd time offset from scintillator strip and readout channcl.

$\Delta \mathrm{T}_{\text {jump }}\left(t>t_{\text {jump }}\right)=$ shift in time offset due to hardware swap at time $t_{\text {jump }}$.

$\Delta \mathrm{T}_{\text {walk }}(Q)=$ time walk correction as function of pulse height $Q$.

Each type of correction is calculated separately using cosmic muon data. The overall corrections are then subtracted from the measured times to give calibrated times. The procedure for the timing calibration is illustrated in Figure 5.1. The shifts in timing caused by changes in hardware components are first calculated from the data. Corrections are applied for these shifts and an initial set of time offsets is calculated for 
each strip end. These offsets are then used to perform an accurate measurement of the time walk corrections. Finally the time walk corrections are fed back into the timing calibration to re-calculate the timing shifts and timing offsets.

The timing calibration is carried out using a sample of high cnergy cosmic muons selected from the data. Events are required to span $>20$ scintillator planes in order to contain a large number of timing measurements. The events are de-multiplexed (see Section 7.2) to determine which strips have been hit in each event. In order to ensure that the muons are well-measured and lie in a clean environment the events are required to satisfy straight line fits in both the $U$ and $V$ views with $r m s$ deviations of $<1 \mathrm{~cm}$. Using this sclection, an event rate of 750,000 muons/month is obtained, corresponding to $400-500$ hits/strip-end/month.

The straight line fits in each view are combined to give a measurement of the $3 \mathrm{D}$ trajectory of each muon track. This is then used to calculate the crossing points of the muons through each scintillator strip. The times recorded at each strip end are projected back to these crossing points by subtracting the propagation times of photons along measured lengths of optical fibre between the strip ends and the muon crossing points. The muon track times calculated by projecting the measured times on the east and west ends of scintillator strips are given as follows:

$$
\begin{aligned}
\mathrm{T}_{\mu}^{L} & =\mathrm{T}^{L}-\frac{n}{c} \mathrm{~L}^{L} \\
\mathrm{~T}_{\mu}^{W} & =\mathrm{T}^{W}-\frac{n}{c} \mathrm{~L}^{W}
\end{aligned}
$$

where:

$\mathrm{T}_{\mu}^{E}=$ muon time projected from east strip end.

$\mathrm{T}^{E}=$ measured time at cast strip end.

$\mathrm{L}^{E}=$ length of fibre between muon trajectory and east strip end.

$\mathrm{T}_{\mu}^{W}=$ muon time projected from west strip end.

$\mathrm{T}^{W}=$ measured time at west strip end.

$\mathrm{L}^{W}=$ length of fibre between muon trajectory and west strip end.

$n=$ refractive index of fibre.

$c=$ velocity of light. 
Each muon track is down-going and travels at a velocity close to the speed of light. The time at distance $S$ along the muon track is parameterized as follows:

$$
\begin{aligned}
& \mathrm{T}_{f i t}^{E}(S)=\mathrm{T}_{f i t}^{E_{1}}(0)+\frac{1}{c} S \\
& \mathrm{~T}_{j i l}^{W}(S)=\mathrm{T}_{\text {fil }}^{W}(0)+\frac{1}{c} S
\end{aligned}
$$

where:

$\mathrm{T}_{f i l}^{E_{i i}}(S)=$ fitted time on cast side at distance $S$ along track.

$\mathrm{T}_{f i t}^{W}(S)=$ fitted time on west side at distance $S$ along track.

Using this parameterization, charge-weighted timing fits are applied on the east and west sides of the detector for each muon track. The time residuals from these fits are then used to calculate sets of calibration constants. The constant time offset associated with a strip is given by the mean residual from timing fits through that strip:

$$
\Delta \mathrm{T}_{0}=\left\langle\mathrm{T}_{\mu}-\mathrm{T}_{\text {fit }}\right\rangle
$$

Using the same method, the time walk correction is given by the mean residual as a function of pulse height:

$$
\Delta \mathrm{T}_{\text {walk }}(Q)=\left\langle\mathrm{T}_{\mu}(Q)-\mathrm{T}_{f i t}\right\rangle
$$

The calibration constants are tuned itcratively by fecding them back into the data, repeating the fits, and then incrementing the constants by the new residuals.

\subsubsection{Measuring the Refractive Index}

The refractive index of the WLS fibre is an essential ingredient of the time calibration procedure. The effective refractive index in the fibre is given by:

$$
n=c \frac{\Delta T}{\Delta L}
$$

where:

$L=$ propagation distance of signal along fibre.

$T=$ propagation time of signal along fibre. 
The refractive index can be calculated from cosmic muon data 11sing double-cnded strips on straight muon tracks. The difference $\mathrm{T}^{E}-\mathrm{T}^{W}$ between the times recorded at each strip end is plotted against the difference $L^{E}-L^{W}$ between the lengths of optical fibre traversed by photons on each side of the detector. The refractive index is calculated from the gradient of a straight line fit through this plot as illustrated in Figure 5.2.

A bias is introduced into this measurement by time walk effects. For strips where $\mathrm{L}^{E_{s}}-\mathrm{L}^{W}<0$, the mean pulse height is greater on the east side of the detector, giving smaller time walk delays on this side and skewing $\mathrm{T}^{E}-\mathrm{T}^{W}$ towards larger negative values. For strips where $\mathrm{L}^{E}-\mathrm{L}^{W}>0$, the mean pulse height is greater on the west side of the detector, giving smaller time walk delays on the west side and skewing $\mathrm{T}^{E}-\mathrm{T}^{W}$ towards larger positive values. This causes the measured refractive index to become an over-estimate of the true refractive index. In order to remove this bias, a threshold is applied to the pulse heights at each strip end. Figure 5.3 shows the measured refractive index plotted against the pulse height threshold. As the pulse height threshold is increased the measured refractive index flattens out to a value of 1.77 .

\subsubsection{Hardware Changes}

Changes in Far Detector readout components occur on a weckly basis. The new components have different characteristic time offsets, causing the timing calibration to shift as each new component is installed. There is insufficient data to perform a full strip-tostrip calibration after each hardware change. Therefore the relative timing shift between the old and new time offsets are measured for each new readout component and timing corrections are applied to the channels read out by the new component.

Timing shifts are identified by analysing the east-west differences between the projected times along muon tracks calculated from the measured times on the east and west sides of the detector. A hardware change on one side of the detector will cause a sudden shift in the east-west difference since the new hardware component will have different time offsets. The mean east-west difference is calculated for each pair of opposite PMTs in the detector after each run. These differenes are then analysed to identify timing shifts between runs. Shifts can be resolved at the level of 0.5 ns. Once a shift has been identified, the side of the detector where the shift took place must be located. Separate timing fits are applied to the east and west sides of the detector using data taken before 


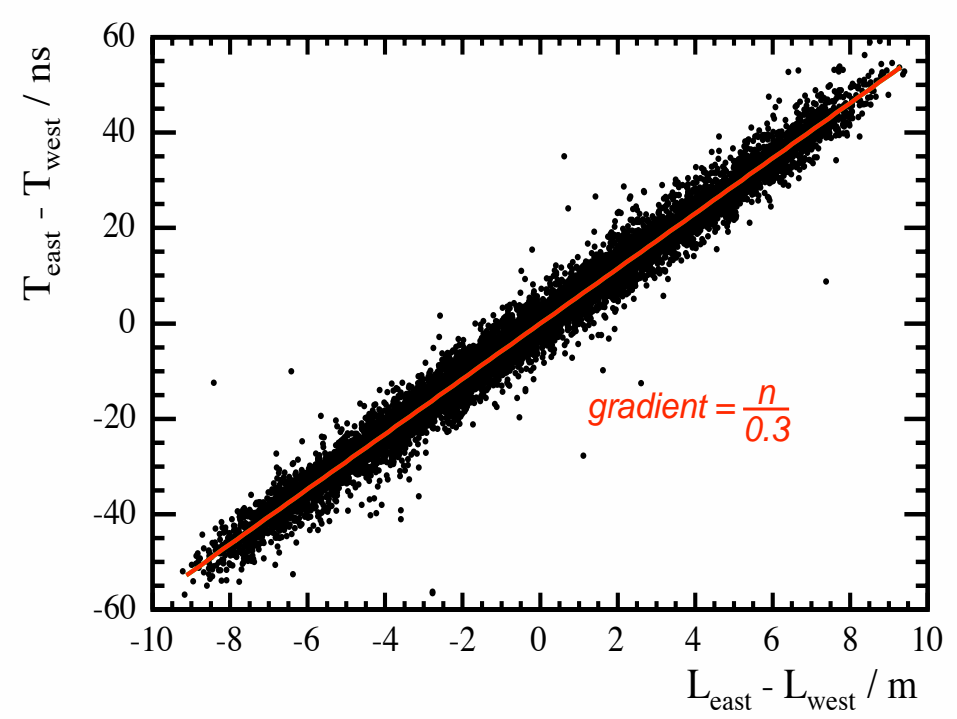

Figure 5.2: Measurement of the refractive index of WLS fibre. The eastwest time difference is plotted against the east-west fibre length difference for strips on muon tracks containing more than 500 ADCs at each end. The effective refractive index is calculated from the gradient of a straight line fit to this distribution.

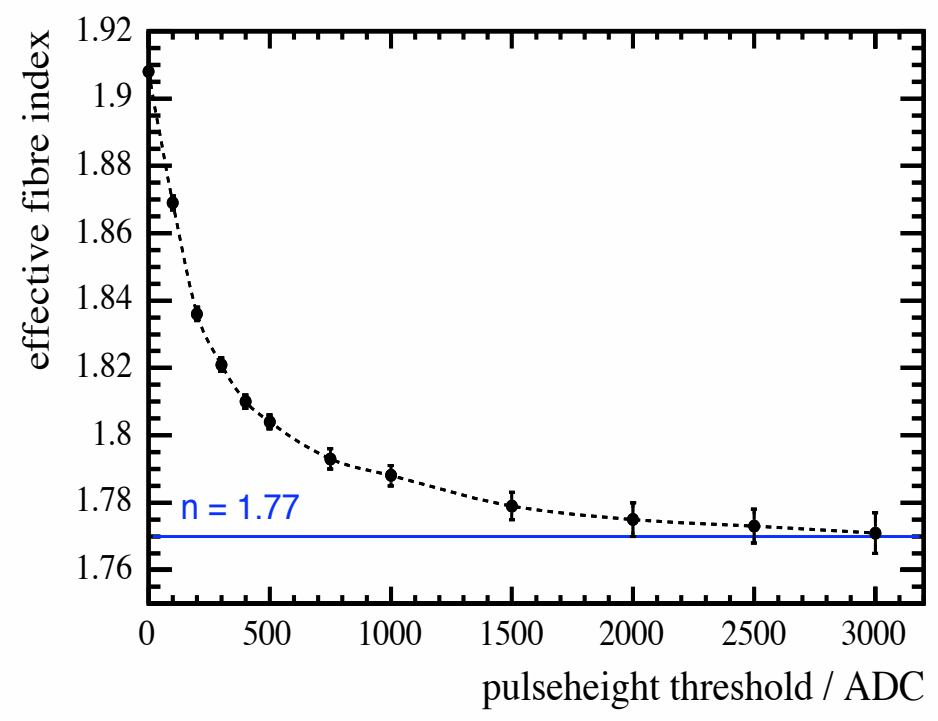

Figure 5.3: Measured refractive index versus pulse height threshold. As the applied pulse height threshold increases, the time walk effects decrease and the refractive index tends to a constant value of $1.7 \%$. 

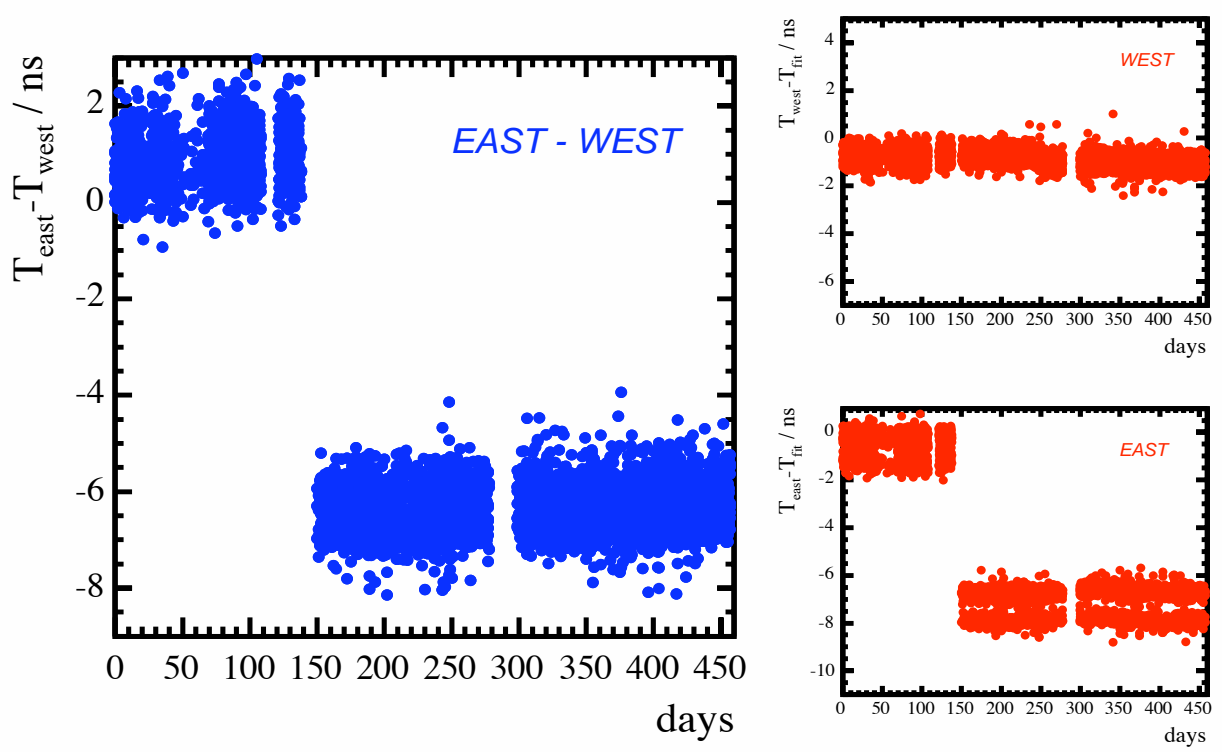

Figure 5.4: (Left) The east-west shift resulting from the replacement of a $V F B$ on the east side of the detector. (Right) The mean residuals from separate timing fits to the east and west sides of the detector. A shift is observed on the east side of the detector, matching the east-west shift.
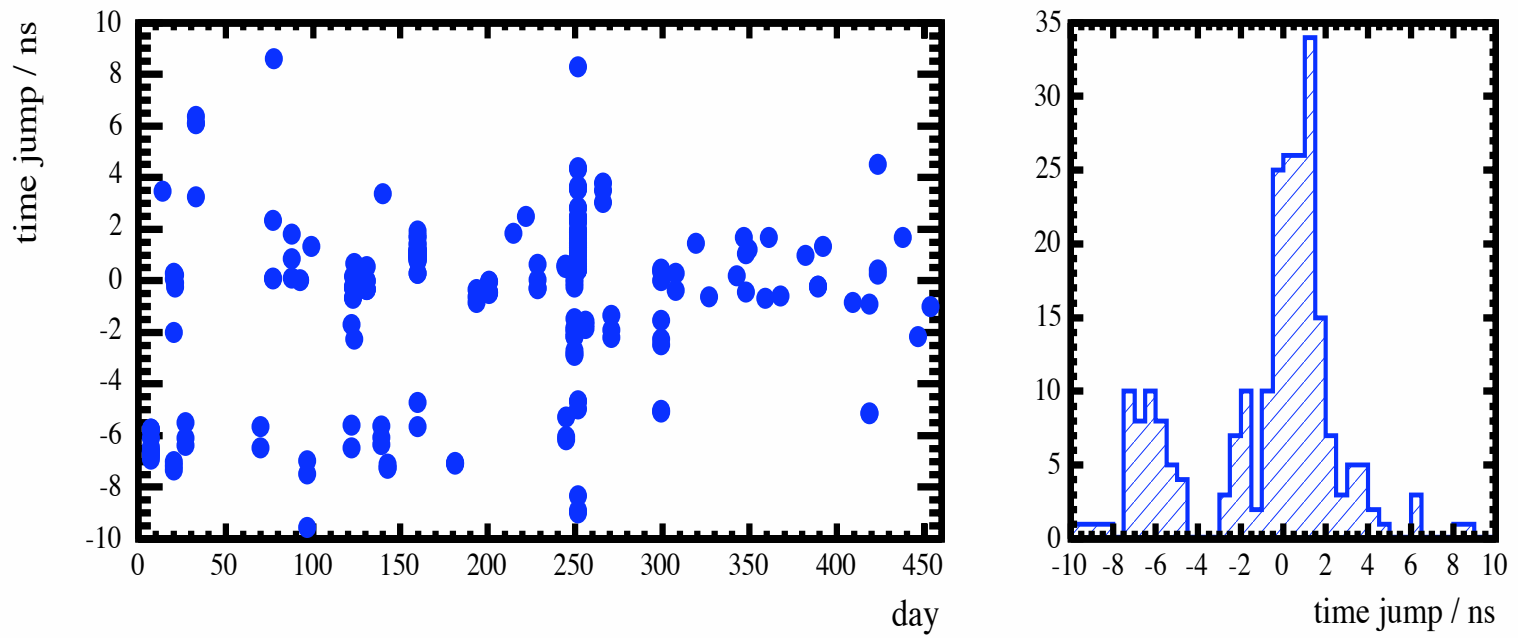

Figure 5.5: (Left) Chronology of time jumps between 1st August 2003 and 31st October 2004. (Right) Distribution of time jumps. 
and after the observed shift. The mean residual for each readout channel is then calculated from these fits. A shift should be observed on the side of the detector where the shift took place, and the size of the shift should match the east-west shift.

As an example, Figure 5.4 shows the effect of replacing a VFB on the cast side of the detector. The east-west time difference is plotted against time for the VA chips on the VFB. After 150 days the VFB is replaced. As a result of this change the east-west difference shifts by $-7 \mathrm{~ns}$. When the mean residuals from timing fits on each side of the detector are calculated, no shift is observed on the west side of the detector but a -7 ns shift is observed on the east side of the detector matching the east-west shift.

Calibration corrections are applied to account for these timing shifts. The size of the correction is given by the measured shift in the east-west time difference. In the above example, a correction of $+7 \mathrm{~ns}$ is required to correct the timing shift caused by the VFB swap. Figure 5.5 shows distributions of all the timing shifts identified in Far Detector data between 1st August 2003 and 31st October 2004. A total of 240 timing shifts are obscrved in the data.

\subsubsection{Time Walk Corrections}

The time walk delay is calculated as a function of the ADC pulse height. Timing fits are applied to muon tracks using only the hits with pulse heights greater than 5000 ADCs where the time walk effects are considered to be small. The time walk delay is then found by calculating the mean time displacements from these fits as a function of ADC using the hits with pulse heights less than 5000 ADCs. The mean time walk delay is parameterized as follows:

$$
\frac{\Delta \mathrm{T}_{\text {walk }}}{\mathrm{ns}}=20.66-2.0178\left(\ln \frac{Q}{A D C}\right)-0.2337\left(\ln \frac{Q}{A D C}\right)^{2}+0.0218\left(\ln \frac{Q}{A D C}\right)^{3}
$$

Figure 5.6 shows the mean time walk and paramaterization of time walk as a function of pulse height. The residuals from the parameterization are also shown as a function of pulse height. The mean time walk is fitted to within \pm 0.2 ns over the full range of ADC values. Typical pulse heights of 300 ADCs are produced by the passage of minimum ionizing charged particles through the scintillator. For signals of this magnitude, a time walk correction of 5 ns must be applied to the measured times. 


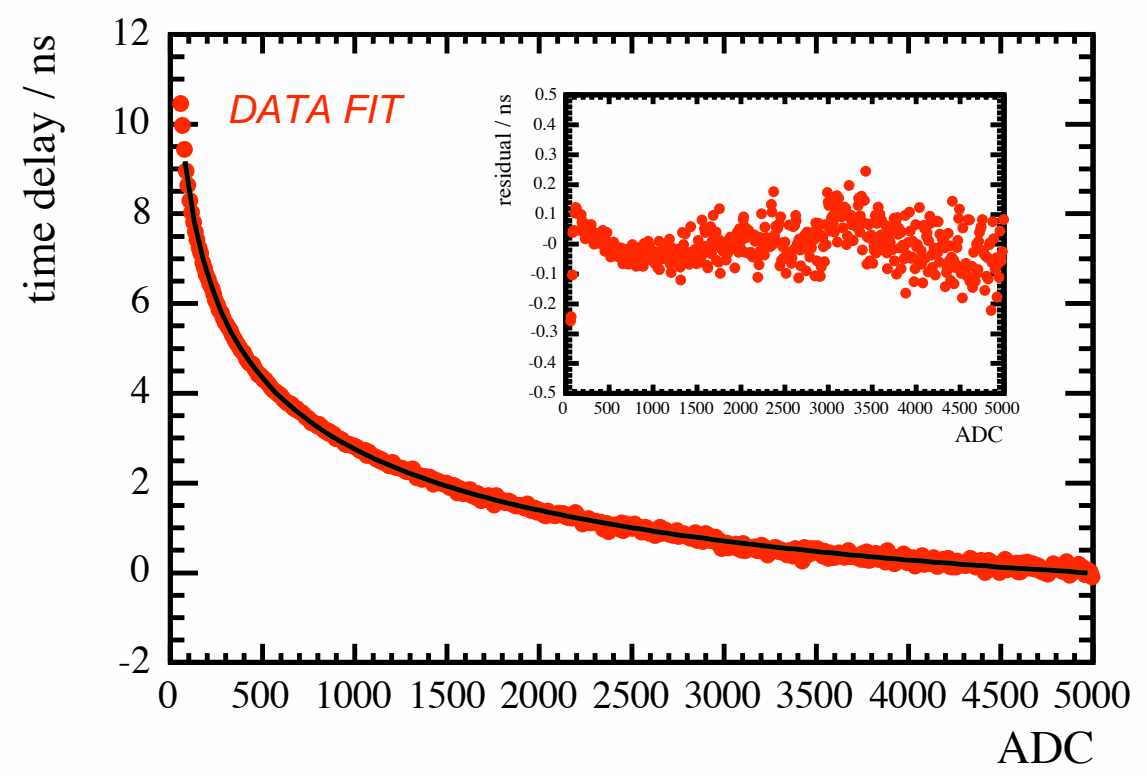

Figure 5.6: The time walk delay as a function of pulse height.

\subsubsection{Strip-to-Strip Calibration}

Once timing corrections have been applied to account for hardware changes and time walk effects, the time offsets between each strip end are calculated. The east and west sides of the detector are calibrated independently. A single east-west offset is then calculated in the final stage of calibration. The calibration of each side of the detector is carried out in two stages. The readout electronics are first calibrated at the PMT and VARC level. After this initial calibration of the timing system, a full strip-to-strip calibration of the timing system is carried out.

The calibration of the readout electronics is carried out by calculating the offsets between the PMTs in each VARC and then calculating the offsets between each VARC. The PMT calibration is carried out for each VARC using muon tracks that pass through all the planes read out by the VARC. Timing fits are applied to the tracks using only the hits read out by the VARC. The time offsets between each PMT in the VARC are then given by the mean residuals from these fits. The VARC calibration is carried out by calculating the offsets between pairs of adjacent VARCs using muon tracks that pass through all the planes read out by both VARCs. Timing fits are applied to the tracks, first using only the hits on the first VARC, and then using only the hits on the second 

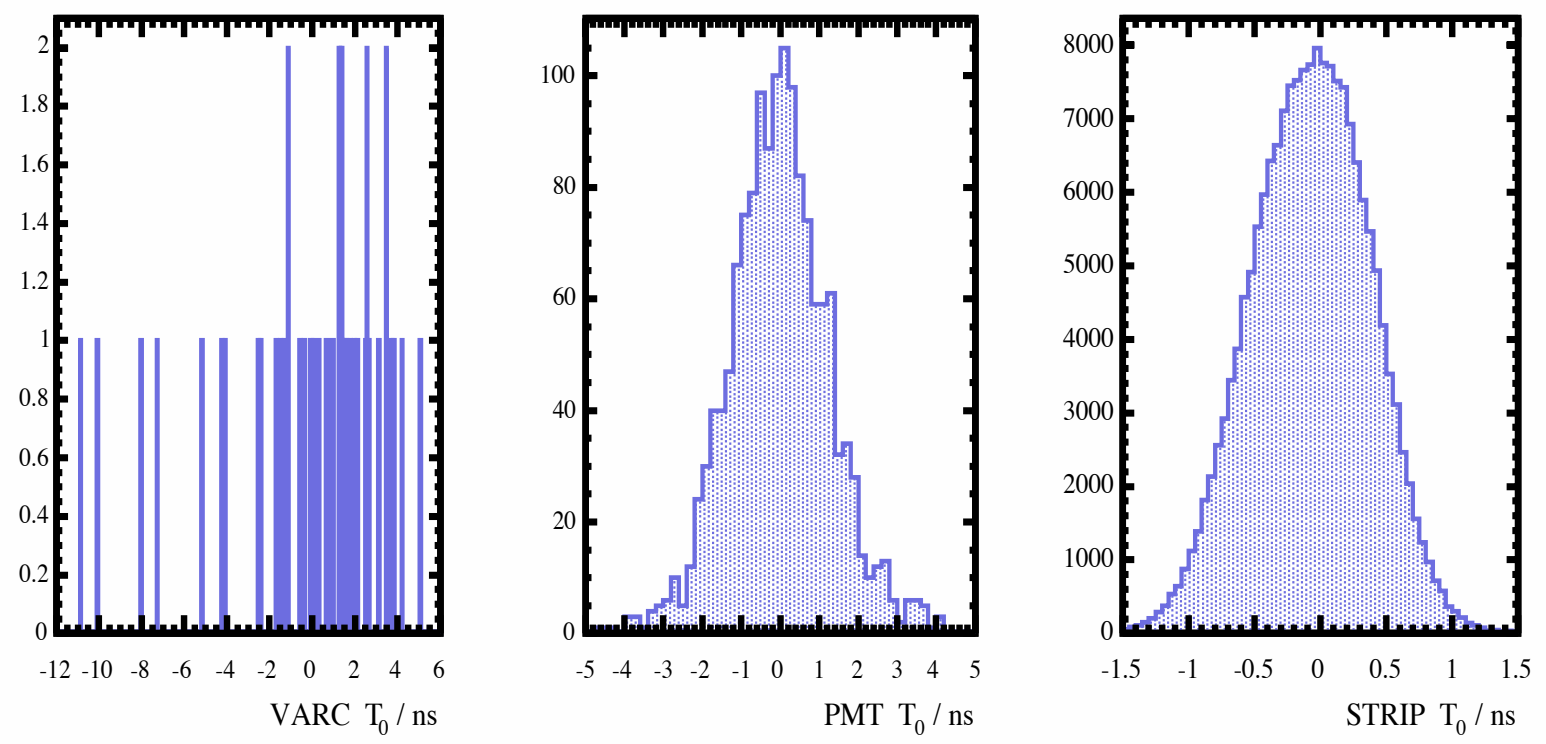

Figure 5.7: Timing offsets for VARCs, PMTs and strip ends.

VARC. The timing fits are projected to the boundary between the VARCs, and the mean time difference is calculated at the VARC boundary. The VARC calibration constants are calculated from the mean time differences between pairs of adjacent VARCs by constraining the mean calibration constant to be zero. The overall calibration constant for each readout channel is given by the sum of the PMT and VARC calibration constants.

Once the time offsets between readout channels have been calibrated, the time offsets between strip ends are calculated. Timing fits are applied to muon tracks using the hits from each side of the detector in turn. The calibration constants for each strip end are given by the mean residuals from these fits. The calibration constant for each strip end is then given by the sum of the time offsets for the strip end and its readout channel.

Figure 5.7 shows the distributions of calibration constants for VARCs, PMTs and strip ends. The largest time offsets occur for VARCs whose calibration constants vary in the range $-12 n s \rightarrow+6 n s$. This scatter is due to the large constant variations in the propagation time of the signals sent from the TCU to the TRCs, and the signals sent from the TRC to the VARCs. The time offsets for the PMTs in each VARC vary in the range $-3 n s \rightarrow+3 n s$. This scatter is due to variations in the rise time of dynode signals in each of the VFBs and propagation time of signals sent between the VFBs and 
VARCs. The time offsets calculated for each strip end vary in the range $-1 n s \rightarrow+1 n s$. This small scatter is due to variations in the rise time of dynode signals within each P.T and small residuals in the measured lengths of the readout fibres.

The final stage of the calibration chain is to calculate the overall time difference between the east and west sides of the detector. This is carried out using double-ended strips on muon tracks. The measured times at the east and west strip ends are projected back to the track and the mean cast-west time difference is calculated. A correction is then applied to shift this time difference to zero.

\subsubsection{Validating the Calibration}

Since the cast and west sides of the detector are calibrated independently, the performance of the calibration can be validated by measuring the time differences between the east and west strip ends. The validation is carried out using double-ended strips on muon tracks. The measured times at each strip end are corrected for hardware changes, time walk delays, and strip-to-strip time offsets. Fibre length corrections are then applied at cach strip end to project the measured times back to the muon track. The mean difference between the times projected from each strip end is calculated. The spread in the mean east-west time differences gives a measure of the accuracy of the calibration. In order to test the stability of the calibration over time, the data is divided into eight blocks of approximately two months and the mean east-west differences are calculated for each block of data.

Figure 5.8 shows the mean east-west time difference plotted as a function of plane number before and after the timing calibration. The uncalibrated time differences vary in the range $-20 \mathrm{~ns} \rightarrow+10 \mathrm{~ns}$. The time differences appear to slope upwards for cach group of planes read out by a common pair of VME crates. This is because the timing signals generated by the TRCs in each VME crate propagate in opposite directions on opposite sides of the detector so that the smallest time offsets on the east side correspond to the largest time offsets on the west side. This creates a "sawtooth" effect in the eastwest time difference as a function of plane number. The timing calibration flattens out the time offsets between strips ends and shifts the mean east-west time difference to zero. The calibrated time differences vary in the range $-4 n s \rightarrow+4 n s$ and are strongly peaked around zero. The small number of outlying points are caused by readout components with malfunctions that produce a small degree of instability in the measured times. 

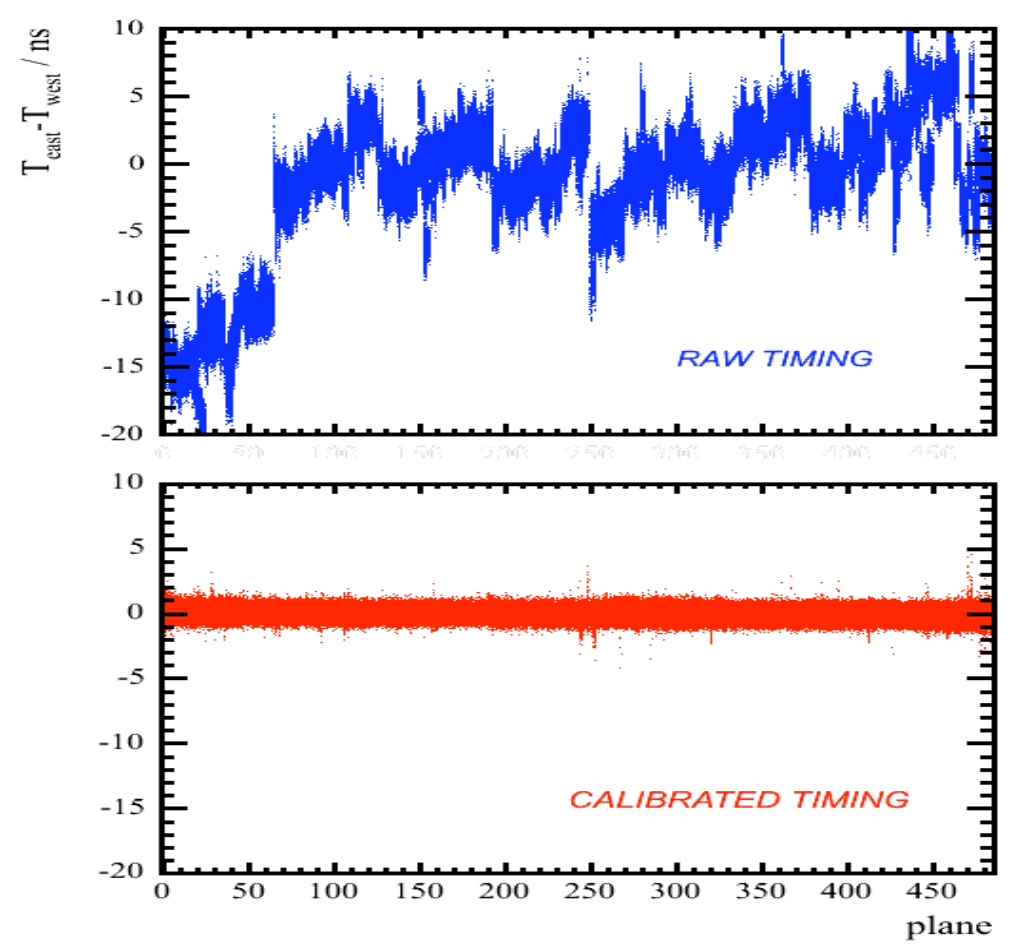

Figure 5.8: Average east-west time difference for each strip plotted as a function of the plane number before and after the timing calibration.

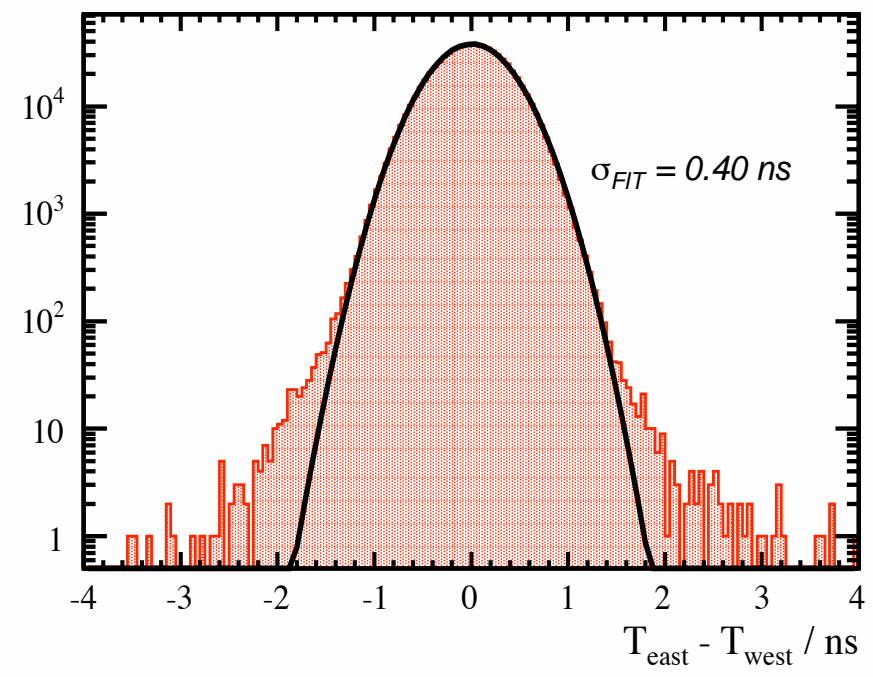

Figure 5.9: Distribution of calibrated east-west differences for all strips. The distribution is found to be a good fit to a Gaussian of width $0.40 \mathrm{~ns}$. 


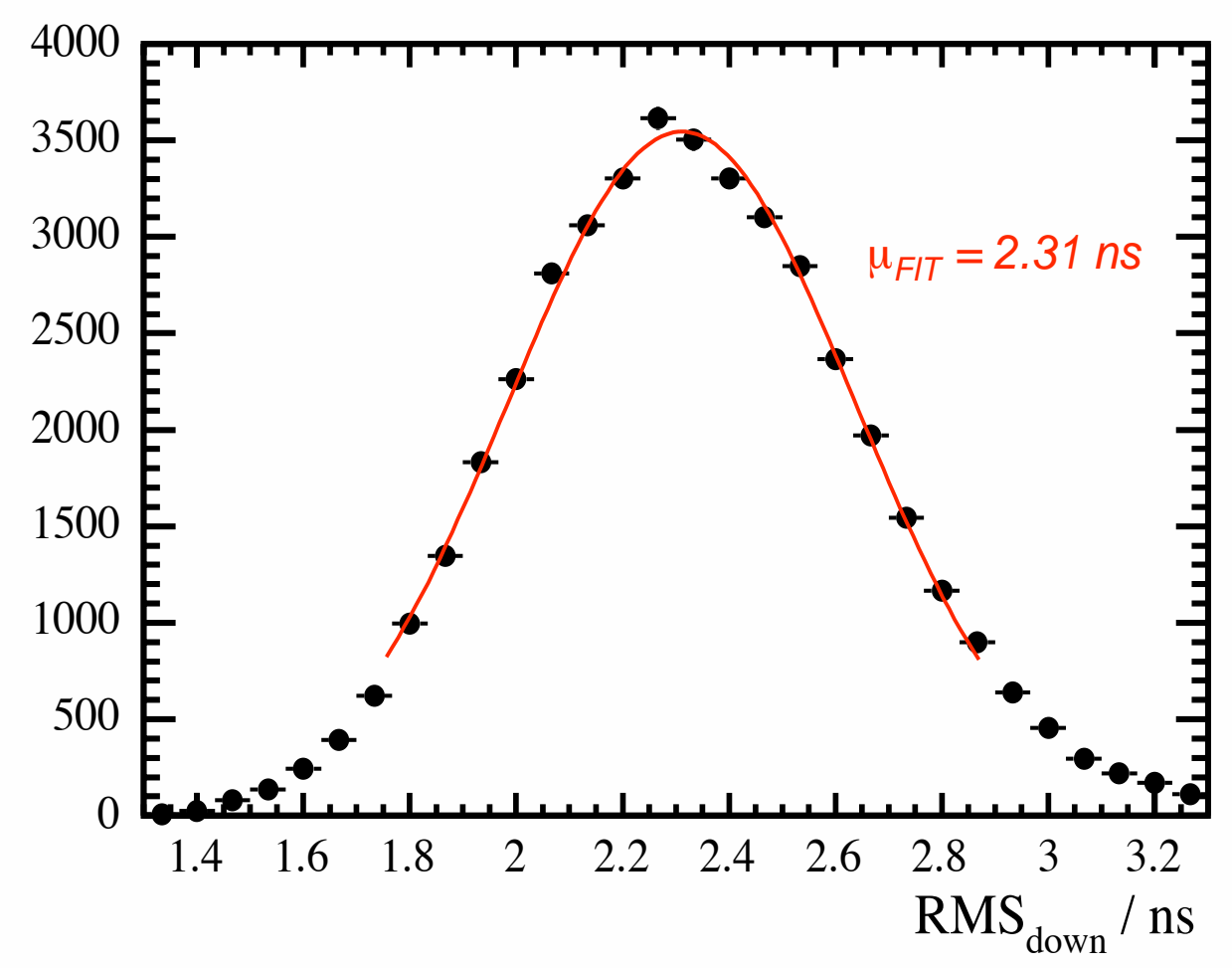

Figure 5.10: Distribution of rms deviations for muon timing fits.

Figure 5.9 shows the distribution of calibrated east-west time differences for each strip and each set of data. A Gaussian fit to this distribution gives a rms of 0.40 ns. The mean statistical error on each east-west time difference is calculated to be 0.06 ns. This is small compared to the overall rms of the east-west time differences, implying that the rms is dominated by the errors in the timing calibration. The rms of the east-west time differences combines in quadrature the mean calibration errors on the east and west sides of the detector. An estimate of the mean calibration error for a single strip end is therefore given by: $\sigma_{\Delta \mathrm{T}}=0.40 / \sqrt{2}=0.28 \mathrm{~ns}$. This implies that the timing system has been successfully calibrated at the sub-nanosecond level.

The overall resolution of the timing system is determined by applying timing fits to the calibrated times on each muon track. The resolution is given by the mean rms deviation of these timing fits. Figure 5.10 shows the distribution of all $\mathrm{rms}$ deviations. A Gaussian fit to this distribution gives a resolution of $2.31 \pm 0.03 \mathrm{~ns}$. 


\subsection{Pulse Height Calibration}

Pulse height information is used to determine the energy of particle showers in the Far Detector. In order to measure the shower energy accurately, the detector must be calibrated to create a uniform and linear response across the readout channels. Each channel has a characteristic response curve which must be measured by the detector calibration systems. There are two calibration systems in place at the Far Detector:

- Light Injection System: A system of Light Injection (LI) [87] is used to measure the lincarity and drift of the PMTs and their readout electronics. A schematic of the LI system is shown in Figure 5.11. Controlled quantities of light are injected from a series of UV LEDs into the WLS fibres at the end of each scintillator strip. The light follows the same optical path as real signals and is digitized in the same way by the front-end electronics. The light is also injected into a set of highly stable and linear PIN diodes which monitor the light level. By varying the amount of injected light, the response of each readout channel can be mapped out. The LEDs are housed in Pulser Boxes. Each box is paired with a VME crate, and injects light into the planes read out by that VME crate. Light is injected at a rate of $50 \mathrm{~Hz}$ to ensure rapid accumulation of data. To distinguish the LI pulses in the data, each Pulser Box houses an additional LED which pulses a dedicated trigger PMT (TPMT). The TPMT hits are read out and digitized by the VA electronics and provide unique identifiers for the LI pulses.

- Charge Injection System: A system of Charge Injection (CI) [88] is used to measure the linearity and drift of the readout electronics. Each VA chip has an in-built CI circuit which allows controlled amounts of charge to be injected as an input to the readout. The charge is then digitized by the VA clectronics in the usual way. By varying the quantity of injected charge, the response of each VA channel can be mapped out.

The LI and CI systems are used to linearize the detector response and monitor drift in the overall response over time. In the lincar region of the readout $(<100 \mathrm{PEs})$ the detector response is characterized by a single constant gain, but for large signals $(>100 \mathrm{PEs}$ ) the full response curve must be measured. Once the response of each readout channel in the detector has been linearized, the strip-to-strip variations across 


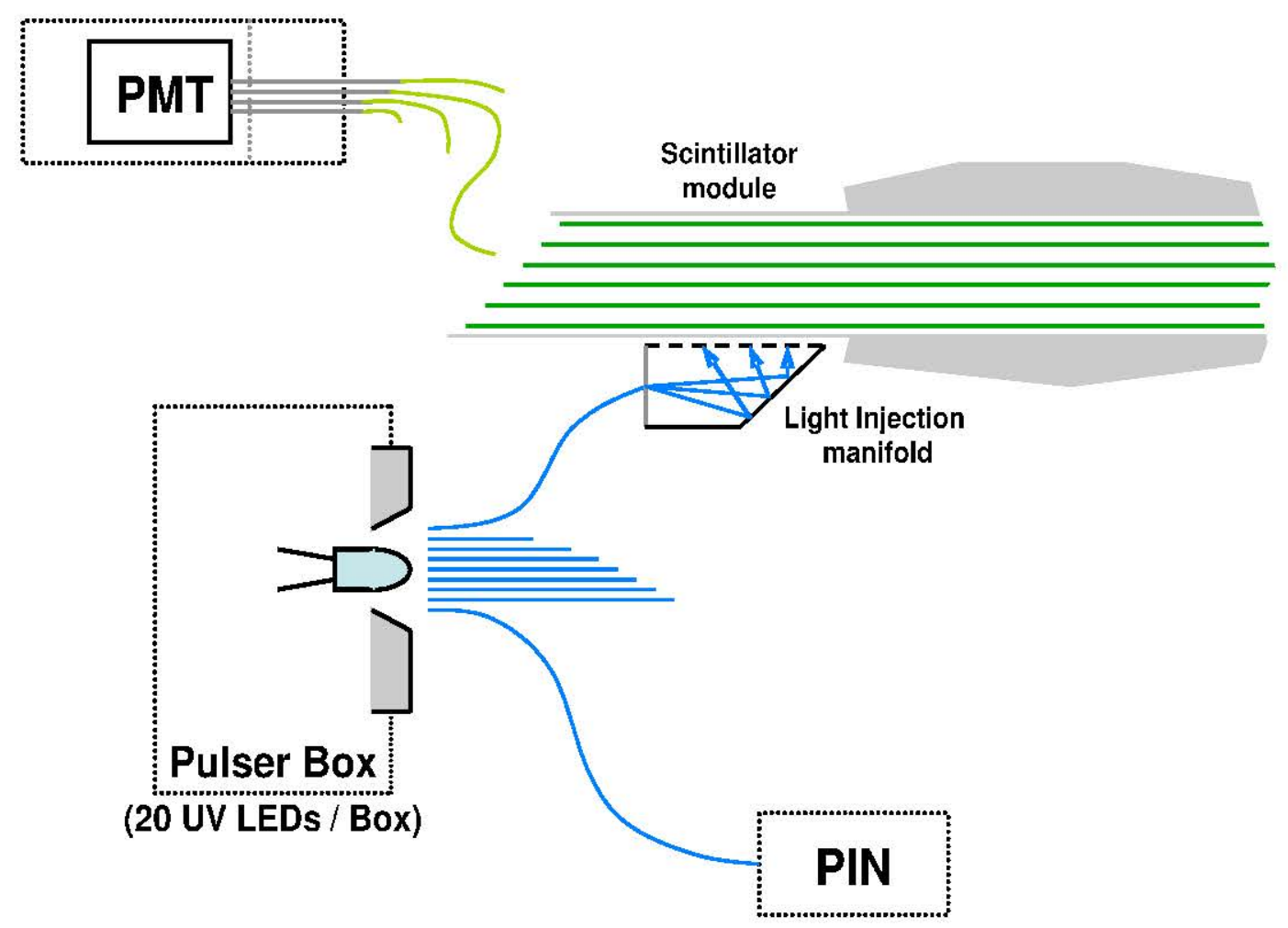

Figure 5.11: The MINOS Light Injection System. A dedicated system of LEDs is used to inject light into the scintillator. The light follows the same optical path as real signals and is digitized by the front-end electronics. The light is also injected into a set of highly linear PIN diodes which monitor the light level. The response of the detector to levels of injected light covering the entire dynamic range of the readout is mapped out.

the detector are measured using cosmic muons. The average energy deposited by muons will be the same for cach strip, so the average pulse height measured at cach strip end will be proportional to the response of the strip. The size of the signal measured at each strip end depends on the amount of attenuation along the strip. The average response measured at the end of a $8 \mathrm{~m}$ length of WLS fibre varies by 11p to a factor of $\sim 3$ depending on the initial position of the signals along the fibre. Therefore the response along each scintillator strip in each module has been mapped using radioactive source prior to the installation of the modules in the detector.

The Far Detector calibration has been developed and tested at the Calibration Detector, and is documented in detail by a number of sources [89] [90] [91]. A summary of the calibration is presented below. 


\subsubsection{Gain Calibration}

For signals of $<100 \mathrm{PE}$, the detector readout is approximately linear [92], producing a constant gain $G$ between the PE and ADC for each readout channel. The LI system is used to determine these gains [93]. Light is injected into the detector at levels chosen to be in the linear regime of the detector readout and the mean and rms ADC from many LI pulses is calculated. Assuming that the initial number of PEs produced at the photo-cathode is dominated by Poisson statistics, the width of the ADC distribution is given by:

$$
\frac{\mu_{A D C}}{\sigma_{A D C}} \simeq \frac{1}{C} \frac{\mu_{P E}}{\sigma_{P E}}
$$

where $C=0.8$ is a factor applied to correct for fluctuations in secondary cmissions on the first PMT dynode which broaden the ADC distribution relative to the PE distribution. Using $\sigma_{P E}=\sqrt{\mu}_{P E}$, the gain $G$ of cach readout channel is calculated as follows:

$$
G=\frac{\mu_{A D C}}{\mu_{P E}} \simeq C \frac{\sigma_{A D C}^{2}}{\mu_{A D C}}
$$

Figure 5.12 shows the distribution of gains in the Far Detector calculated using Equation 5.9. The mean gain of the readout is calculated to be $67 \pm 1 \mathrm{ADC} / \mathrm{PE}$, with a spread of $20 \%$ across the readout channels.

\subsubsection{Linearity Calibration}

For signals of $>100$ PE, the readout becomes non-lincar as the PMTs start to saturate. The LI system is used to convert the ADC scale into a scale linear in light. The LEDs are pulsed at a scries of light levels chosen to cover the cntire dynamic range of the ADC readout. The PMT response is then mapped out using the PIN diodes. Figure 5.13 shows a typical PMT response curve measured by the Light Injection system. The mean ADC measured for the PMT is plotted against the mean ADC measured for the PIN diodes for each light level. The PMT response curve is linear up to 10,000 ADCs and then flattens out as the P.MT saturates.

Although the response of the PINs is known to be highly stable and linear, the PINs are read out and digitized by the VA electronics which are known to be non-linear. Before the PINs can be used to calibrate the non-linearity of the PMTs, the non-linearity of the VA electronics must first be removed. This is achieved using the CI system. Charge 


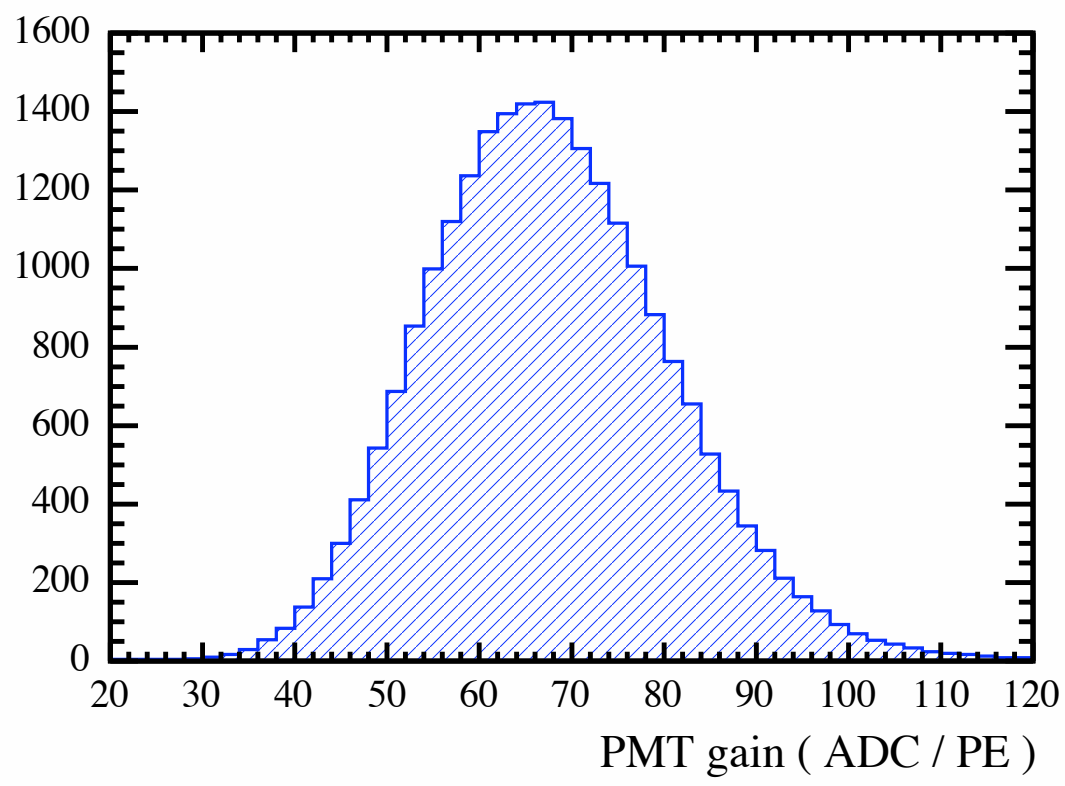

Figure 5.12: Distribution of PMT gains.

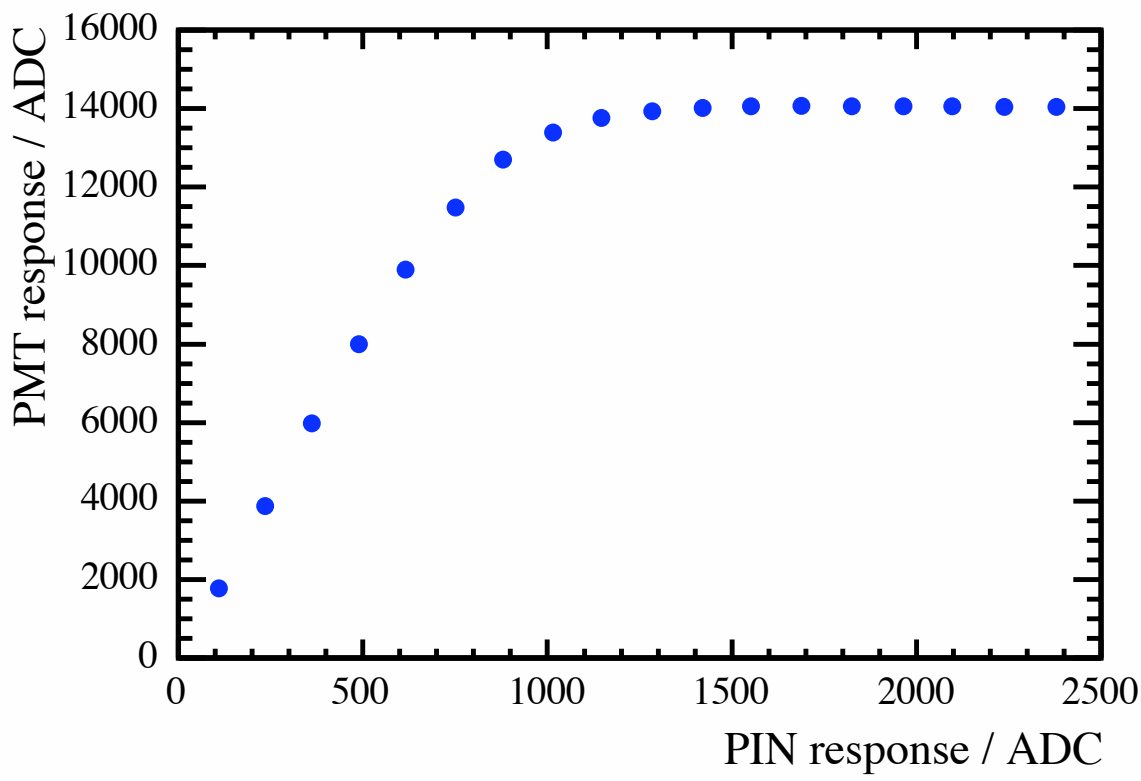

Figure 5.13: Typical PMT response curve. The average PMT response is plotted against the average PIN response for a series of light levels. 
is injected into the electronics at levels covering the dynamic range of the VA readout. The mean response is then measured for each level. Once the PINs have becn lincarized, they are in turn used to linearize the PMTs and their readout electronics.

Variations in the temperature and environmental conditions around the detector can cause the calibration to drift over time. A drift of $1 \% /{ }^{\circ} \mathrm{C}$ in the absolute response and $1 \% /$ month in the relative response of channels has been observed at the Far Detector [94]. The drift in detector response is monitored using the LI system. The LEDs are pulsed in the linear region of the PMTs and the amplitudes of the PMT responses are measured. The PINs are used to remove any variations in the LED light output. A scaling factor is then applied to the amplitude of the detector response to correct for any drift. To ensure that the detector is well calibrated, drift monitoring is continually interspersed with normal data-taking ${ }^{a}$.

\subsubsection{Strip-to-Strip Calibration}

Once the response of each readout channel has been linearized, a full strip-to-strip calibration [95] is performed to normalize the variations in response between each scintillator strips and create a uniform response across the detector. Variations in detector response arise for a number of reasons:

1. Differences in the scintillator light output.

2. Variations in attenuation coefficients of optical fibres.

3. Variations in the transmission efficiency of optical connectors.

4. Scale factors not removed by lincarity calibration (c.g. PIN gain).

The variations between strips are normalized by measuring the response of each strip to ionization energy deposited by cosmic muons passing through the detector. The average energy deposited by muons is the same for each strip and therefore the average pulse height at each strip end is proportional to the response of the strip.

The strip-to-strip calibration is carried out by calculated the average linearized ADC response at each strip end. Each ADC measurement is corrected to account for for muon

\footnotetext{
${ }^{a}$ At the time of this analysis, the linearity and drift calibrations were not yet available.
} 


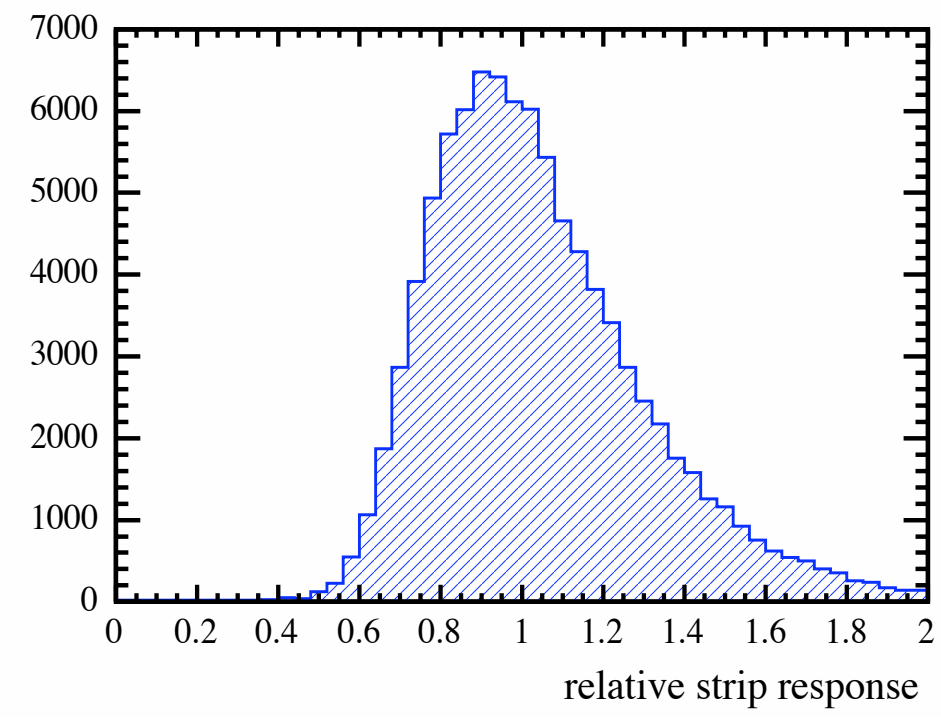

Figure 5.14: Relative response of scintillator strips.

path lengths through the scintillator strips and attenuation of signals in the WLS and clear fibres. Since the mean number of photo-electrons generated by cosmic muons at each strip end is small ( $\sim 5 \mathrm{PEs}$ ), the probability that the signal fluctuates to zero is not negligable. Therefore, additional "zero" corrections are applied to the mean ADC measurements to account for undetected signals. Figure 5.14 shows a normalized distribution of strip-to-strip responses at the Far Detector. There is a 25\% spread in the responses of scintillator strips across the detector. A uniform detector response is obtained by dividing a pulse height at a given strip end by its normalized response.

\subsubsection{Energy Calibration}

The final stage of the calibration is to convert the normalized detector response into an overall energy scale. In order to determine the energy scale, the Calibration Detector has measured the response of the MINOS detectors to different types of particles with known energies [96] [97]. The results have been used to tune the models used in the simulation of particle showers. For this analysis, the overall energy scale is set by measuring the mean response of the Far Detector to cosmic muons and tuning the detector simulation to reproduce this response. The reconstruction of neutrino shower energies is then developed using the tuned detector simulation. 


\section{Chapter 6}

\section{Monte Carlo Simulation}

\subsection{Simulation Overview}

The atmospheric neutrino analysis is developed and tuned using a Monte Carlo (MC) simulation of the Far Detector. The simulation is used to develop the reconstruction and sclection of atmospheric neutrino events. The oscillation analysis is also performed by comparing the data with the expectation. The MC simulation for the atmospheric neutrino analysis is divided up as follows:

- Atmospheric Neutrino Simulation: The flux of atmospheric neutrinos incident on the detector is calculated by simulating the cascade of particles produced by cosmic ray interactions in the atmosphere. The rate of atmospheric neutrino interactions in the detector is calculated using models of neutrino cross-sections.

- Cosmic Muon Simulation: Cosmic muons form the main background to atmospheric neutrinos. The flux of muons incident on the detector is calculated by extrapolating a paramaterization of the flux measured on the surface.

- Detector Simulation: Particles generated in the detector are tracked through the stecl and scintillator. The light emissions in the scintillator and the creation and propagation of photons along the fibres are modelled. The detector front-end instrumentation is simulated to determine the measured signals.

A number of detailed computer models have been developed to simulate the flux of particles incident on the Far Detector and the response of the detector to these particles. These models are described in detail below. 


\subsection{Detector Simulation}

\subsubsection{Simulation of Active Detector}

The principal simulation software used by MINOS is the gminos package [98]. This defines the composition and geometry of the detector, and interfaces to Geant3 [99] for the physics of particle transport through materials. gminos reads in a list of particle four-momenta, inserts these particles into the detector, and outputs a record of encrgy deposits in the active scintillator as determined by the Geant3 simulation.

The active scintillator response is modelled by the PhotonTransport package [100]. This package simulates the creation and propagation of photons in the scintillator and their subsequent conversion to photo-electrons on the PMT photo-cathodes. For each recorded energy deposit in the scintillator, PhotonTransport generates a number of blue photons according to Birks' Law as follows [101]:

$$
N=C \frac{L_{0}}{C_{0}} \frac{d E}{1+k_{B} \frac{d L}{d x}}
$$

where:

$d E=$ energy deposited in the scintillator.

$L_{0}=$ light output of the scintillator.

$C_{0}=$ calibration constant applied to correct for the strip response.

$C=$ overall normalization used to tune the light output.

$k_{B}=$ Birks' constant (taken to be $0.133 \mathrm{~m} \mathrm{GeV}^{-1}$ ).

Some of the blue photons are captured by the WLS fibres and converted into green photons. The green photons are distributed along the WLS fibre using a set of PDFs relating the positions and times of green photons to the positions and times of the bluc photons. The PDFs are constructed from detailed simulations of photon cmission, propagation and absorption in the scintillator strips and WLS fibres. Each green photon is tracked individually down the WLS and clear fibres, accounting for attenuation in the fibres. The photons are converted into photo-clectrons at the PMT photo-cathodes using a flat probability of $13 \%$, equal to the measured quantum efficiency of the M16 PMTs. Additional single photo-electrons are also generated according to the measured rates to simulate background noise in the detector. 


\subsubsection{Simulation of M16 PMTs and VA Electronics}

The Far Detector Front-End instrumentation is simulated by the DetSim package [100]. This provides models for the amplification of PEs through the M16 PMTs, the digitization of charge by the VA electronics, and the implementation of DAQ triggers.

The total number of PEs on a photo-cathode is determined by summing the individwal PEs generated by the PhotonTransport simulation on that photo-cathode. Optical crosstalk between adjacent PMT pixels is taken into account by redistributing the PEs on each PMT photo-cathode according to a probability matrix calculated from the results of M16 bench studies [102]. The amplification of cach signal through a PMT is simulated in two stages. The signal is initially amplified through the first pair of dynodes and the resulting charge is smeared to account for fluctuations due to secondary dynode emissions. The final charge on the photo-anode is then calculated using the measured response PMT curve. The dynode trigger threshold is set at a level equivalent to 0.3 PEs, and the dynode trigger time is taken to be the arrival time of the first photon on any of the photo-cathodes on the PMT.

Once the amplification of signals by the PMTs has been simulated, DetSim then models the response of the VA readout electronics. Signals that satisfy the 2/36 VARC trigger are amplified using the measured VA response curves and smeared using the measured pedestal widths. The signals are digitized and hits with $<20$ ADCs are sparsified away. Finally, the digitized charges and times are joined with the channel numbers to create raw detector hits. DetSim replicates the treatment of hits by the TPs and DCP. The 4/5 plane trigger algorithm is applicd to the hits to select interesting physics events. These events are then written out in the same format as real data.

\subsubsection{Data-MC Comparison of Detector Response}

Each detector hit provides a measurement of timing and pulse height. Both these quantities must be simulated correctly in order to minimize the systematic errors in the atmospheric neutrino analysis. The detector simulation contains free parameters used to vary the simulated detector response. These parameters are tuned to bring the simulation of timing and pulse height into agreement with the data. The MC is tuned using cosmic muon tracks that cross $>20$ scintillator planes and satisfy straight line fits with deviations of $<1 \mathrm{~cm}$ in both views. 


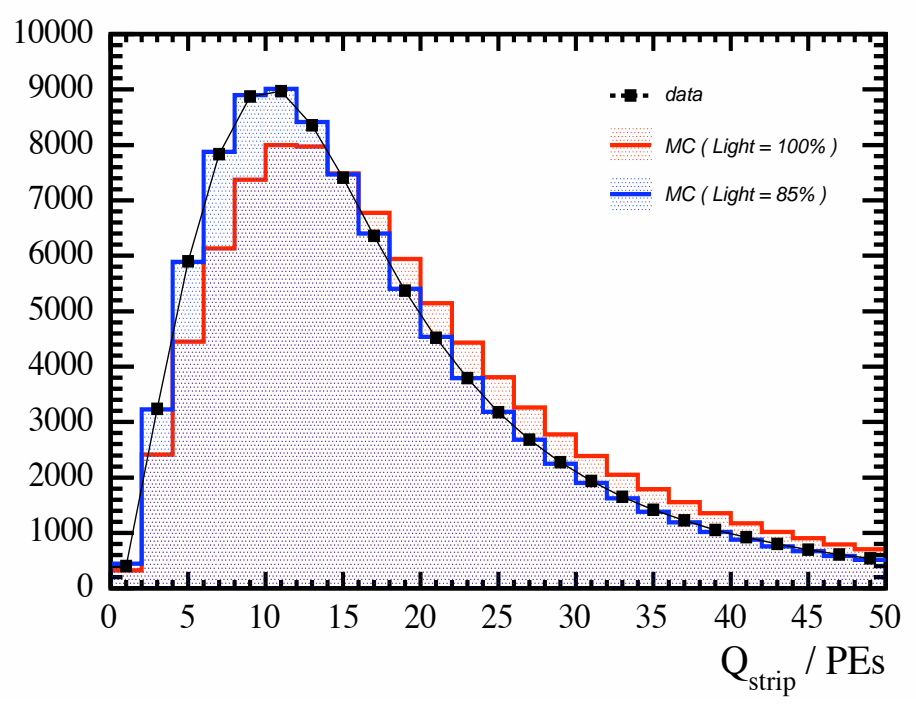

Figure 6.1: Pulse height of double-ended strips on cosmic muon tracks. Data is represented by the black squares, default $M C$ by the red line, and tuned $M C$ by the blue line. An overall normalization of $85 \%$ is applied to the scintillator light output to bring the $M C$ into agreement with the data.

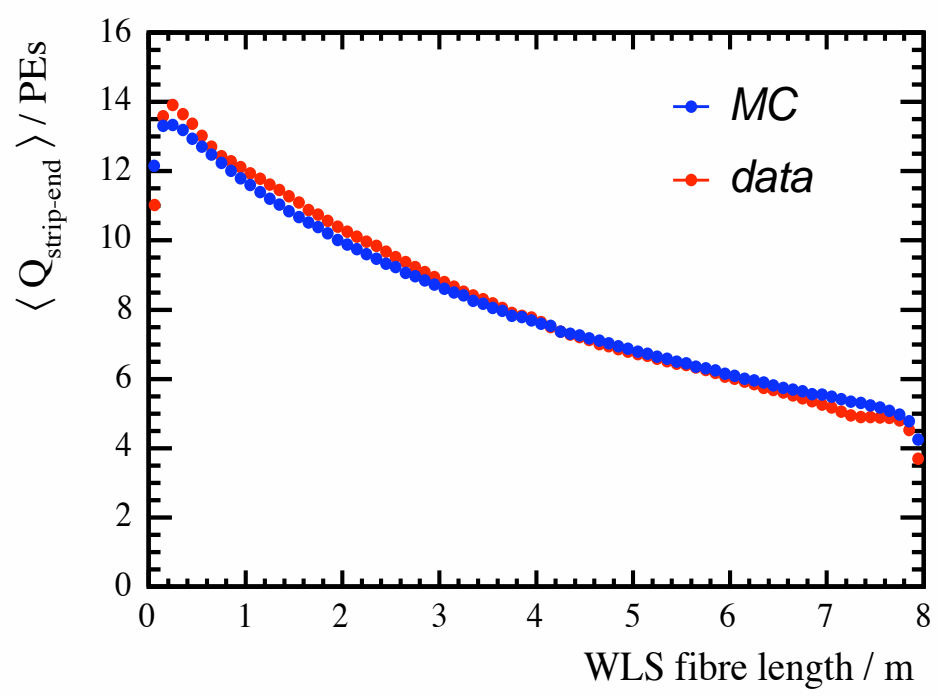

Figure 6.2: Mean pulse height measured at strip ends against muon distance from strip ends. Corrections are applied to account for light attenuation along the clear fibre. MC and data are found to agree to within 10\% over the entire length of the WLS fibres. 


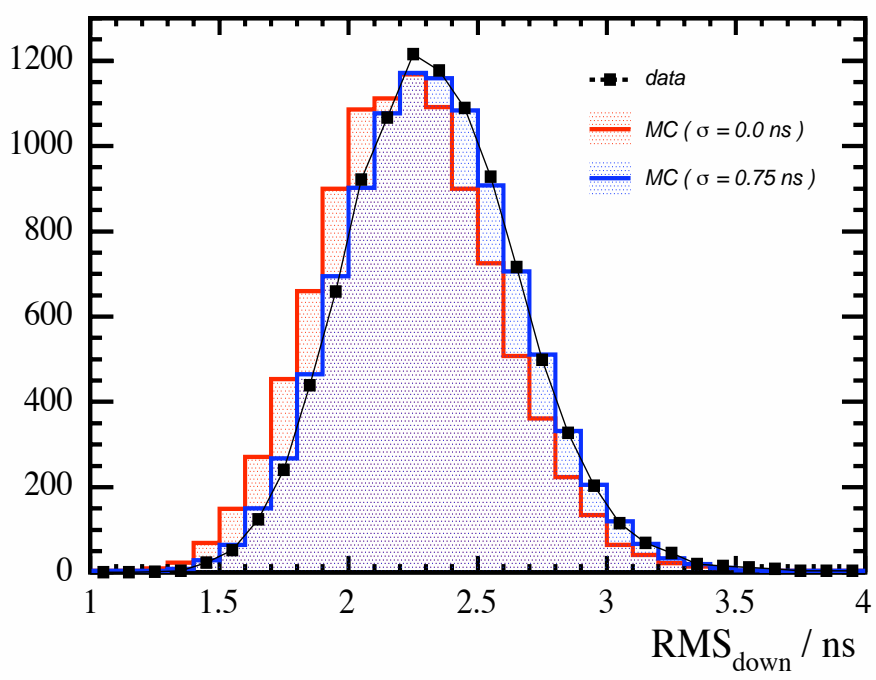

Figure 6.3: The rms deviations of timing fits to cosmic muon tracks. Data is represented by the black squares, default $M C$ by the red line, and tuned $M C$ by the blue line. Measurements of timing are smeared by $0.75 \mathrm{~ns}$ to bring the $M C$ into agreement with the data.

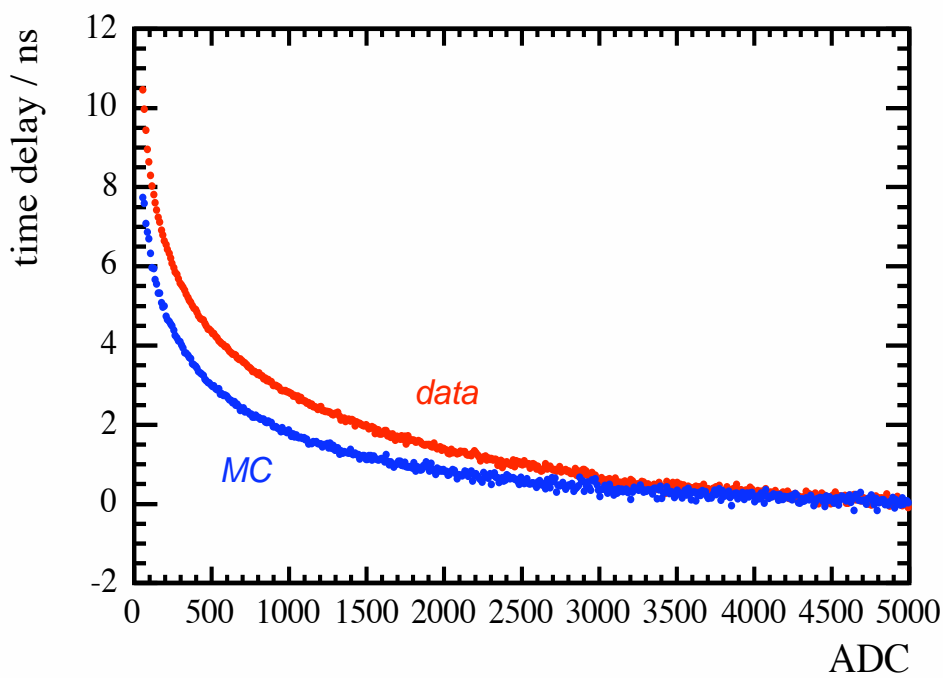

Figure 6.4: The time walk delay as a function of pulse height calculated for data and MC. The time walk correction is lower in $M C$ than data. 
The MC simulation is found to produce larger pulse heights than the data. In the active scintillator model, the number of photons generated as a function of deposited energy is defined according to the current published measurements. However, losses are incurred in the propagation of photons down the fibres which reduce the effective light output. The detector simulation contains a tunable normalization $C$ which controls the overall light output of the scintillator. The value of $C$ is tuned to correctly reproduce the pulse height distribution of cosmic muons. Figure 6.1 shows the distribution of the total pulse height of double-ended strips along cosmic muon tracks, plotted for data and for $\mathrm{MC}$ before and after the pulse height tuning. A value of $C=85 \%$ is required to bring the MC into agreement with the data.

In addition to the overall light output, the attenuation of signals along the WLS fibres must be simulated correctly. Figure 6.2 shows the mean pulse height at each strip end as a function of the distance of the muon from the strip end, plotted for data and tuned MC. The data and MC agree to within $10 \%$ over the entire length of the WLS fibre. The small differences occur because the MC applies constant attenuation lengths for each strip whereas the data represents the average over many attenuation curves. A drop in signal occurs $25 \mathrm{~cm}$ from the strip ends as light starts to escape from the ends of the scintillator before it is captured by the WLS fibres.

The MC simulation is found to have a better timing resolution than the data. The mean $r m s$ deviation of timing fits to muon tracks is calculated to be $2.31 \pm 0.03$ for data and $2.19 \pm 0.03$ for MC. One reason for the difference between data and MC is that the time delays due to the rise of dynode signals in the PMTs are not modelled in the detector simulation. The MC is also perfectly calibrated whereas the data contains a certain amount of scatter in the calibration. A tunable smearing is applied to the MC timing measurements using a Gaussian distribution of width $\sigma$ truncated at $\pm 2 \sigma$. The value of $\sigma$ is tuned to correctly reproduce the mean rms deviation of timing fits to muon tracks. Figure 6.3 shows the distribution of $r m s$ deviations for muon tracks, plotted for data and $\mathrm{MC}$ before and after the $\mathrm{MC}$ tuning. A value of $\sigma=0.75 \mathrm{~ns}$ is required to bring the MC into agreement with the data.

The time walk corrections for MC are calculated in the same way as data. Figure 6.4 shows the time walk as a function of pulse height for data and tuned MC. The time walk is found to be $25 \%$ lower in MC than data. This is because the delays due to the rise of dynode signals in the PMTs are not modelled in the detector simulation. 


\subsection{Cosmic Muon Simulation}

\subsubsection{The Cosmic Ray Flux}

The Earth is constantly bombarded by a stream of cosmic rays which strike the top of the Earth's atmosphere at a rate of $\sim 1000 \mathrm{~m}^{-2} \mathrm{~s}^{-1}$ The vast majority of these particles are ionized nuclei, composed of $95 \%$ protons, $4 \%$ alpha particles and $1 \%$ heavy nuclei [103] [104] [105]. Cosmic rays have been observed over a large range of energies covering at least 14 orders of magnitude from $10^{7}$ to $10^{20} \mathrm{cV}$. Direct measurements of the cosmic ray spectrum have been performed up to $10^{12} \mathrm{eV}$ by a series of air-borne [106] [107] [108] and space-borne [109] experiments. Figure 6.5 shows one such collation of results for the major components of the primary flux [105]. At higher cnergies, cosmic rays are studied indirectly by observing the large air showers produced by their interactions in the atmosphere using arrays of ground-based detectors [110] [111] [112] [113]. The cnergy of the primary cosmic ray is then inferred from the multiplicity of particles in the air shower or the amount of radiation emitted in the atmosphere. Figure 6.6 shows the "all-particle" spectra measured by these air shower cxperiments $[105]$.

For energies below $10^{10} \mathrm{eV}$, the cosmic ray flux is modulated by the solar wind, a steady stream of charged particles emitted from the surface of the sun whose magnetic ficld shiclds the inner solar system from the flux of low encrgy cosmic rays arriving from outside the solar system. For energies above $10^{10} \mathrm{eV}$, the energy spectrum is observed to follow a power law distribution $N(E) \sim E^{-\gamma}$ over a wide range of energies, with the exponent $\gamma$ measured to be $\gamma \sim 2.7$. Above $5 \times 10^{15} \mathrm{cV}$, this stecpens to $\gamma \sim 3.0$, a feature commonly referred to as the knee of the spectrum. The bulk of cosmic rays below the knec are believed to originate from inside the galaxy, with the power law shape resulting from an acceleration process (e.g. supernova explosions) that approaches its maximum at the knee. Above $3 \times 10^{18} \mathrm{eV}$ the energy spectrum flattens out again, a feature commonly referred to as the ankle of the spectrum. At these cnergies, cosmic rays are longer confined by the galaxy's magnetic field. Therefore, the ankle is believed to represent the cross-over point between cosmic rays produced within the galaxy and cosmic rays of extra-galactic origin [114].

Cosmic rays interact in the atmosphere to produce a cascade of secondary pions and kaons. These secondary particles in turn decay to produce a high flux of cosmic muons. The cosmic muons are typically produced at altitudes of $20 \mathrm{~km}$ in the atmosphere. The 


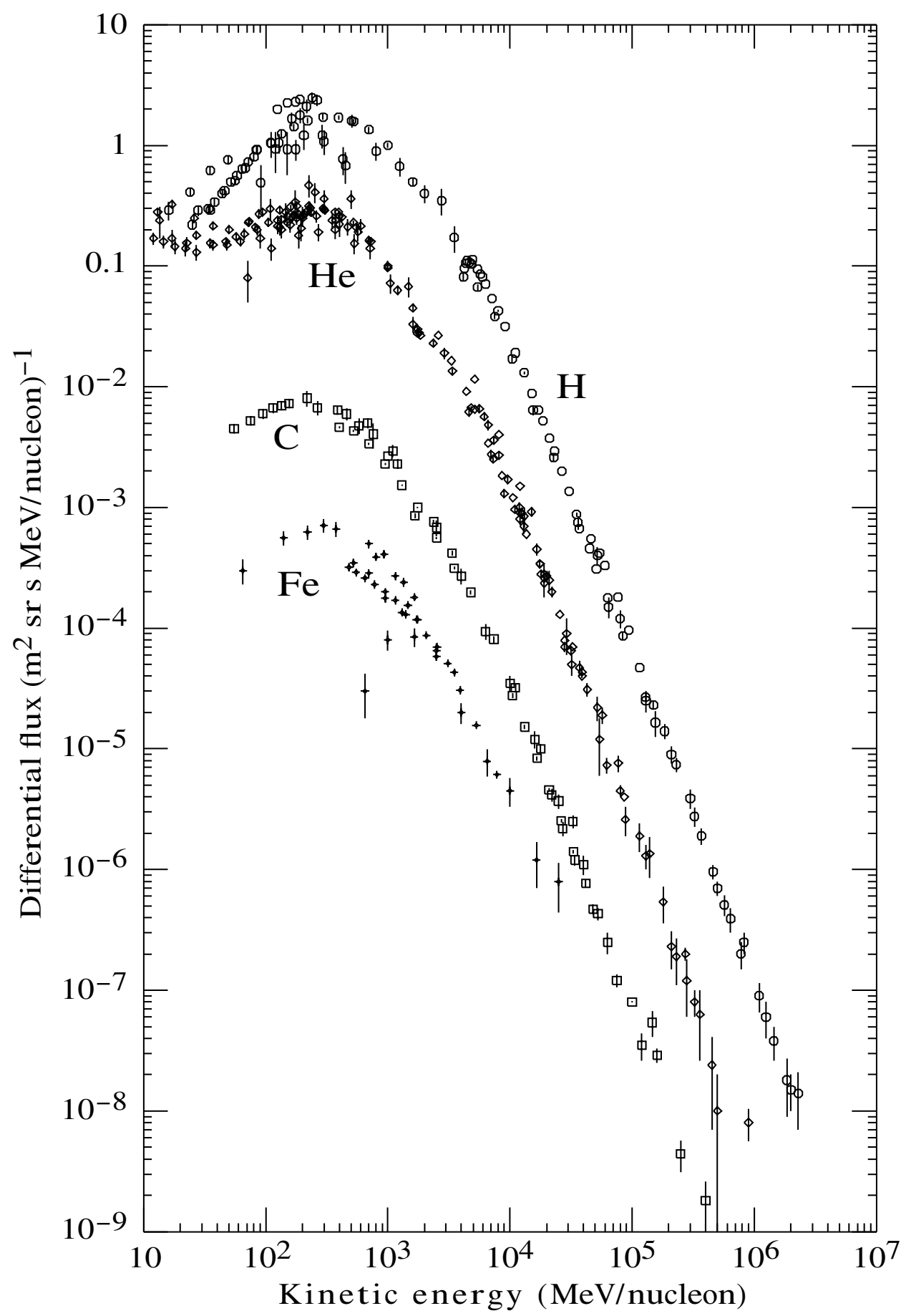

Figure 6.5: The primary cosmic ray spectrum for energies below $10^{12} \mathrm{eV}$. The measured energy spectrum is shown for $\mathrm{H}, \mathrm{He}, \mathrm{C}$ and $\mathrm{Fe}$ [105]. 


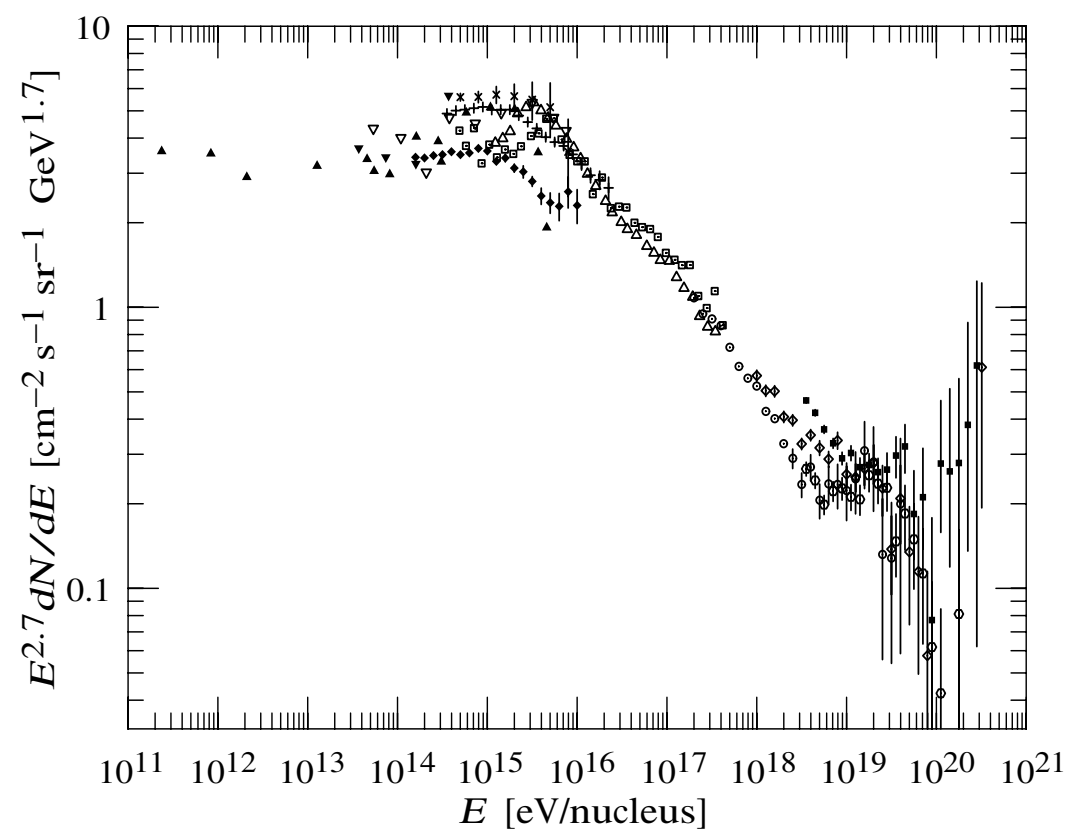

Figure 6.6: The "all-particle" spectrum of cosmic ray interactions above $10^{11} \mathrm{eV}$ observed by air shower detectors [105]. The energy spectrum has been multiplied by $E^{2.7}$ to demonstrate the features of the spectrum.

muon energy spectrum and angular distribution reflects the primary cosmic ray spectrum and the underlying physics of hadron production and decay. The flux of cosmic muons has been measured by a number of experiments [115] [116] [117]. The muon spectrum is observed to fall steeply with energy reflecting the shape of the primary spectrum. The mean energy of muons incident on the surface is measured to be $4 \mathrm{GeV}$, but muons with energies in excess of $100 \mathrm{GeV}$ are observed. Experiments agree on the shape of the energy spectrum, but measurements of the absolute flux differ at the level of $20 \%$.

High energy muons are capable of penetrating to great depths. The cosmic muon flux falls off rapidly with depth as the threshold energy required for muons to penetrate through to that depth increases. At the Far Detector, the flux is reduced by a factor of $\sim 10^{5}$ relative to the surface and cosmic muons are incident on the detector at a rate of $1 \mathrm{~Hz}$. Cosmic muons provide an important tool in performing and validating the simulation and calibration of the detector. They also form the primary background to the atmospheric neutrino signal. Therefore it is important to correctly simulate the flux of cosmic muons incident on the Far Detector. 


\subsubsection{Simulation of Underground Cosmic Muon Flux}

The flux of cosmic muons at the Far Detector is calculated by propagating the cosmic muon flux measured at the surface through a digitized map of the rock overburden above the Soudan cavern [118]. The cosmic muon flux at the surface can be parameterized as follows [119]:

$$
N(E, \cos \theta)=\frac{0.14 E^{-2.7}}{c^{2} \operatorname{sr} G e V s}\left(\frac{1}{1+\frac{1.1 F \cos \theta}{115 \operatorname{GeV}}}+\frac{0.054}{1+\frac{1.1 F \cos \theta}{850 \mathrm{GeV}}}\right)
$$

The parameterization is valid for $E>100 \mathrm{GeV}$ where muon decays can be neglected, and for $\theta<70^{\circ}$ where the curvature of the Earth can be neglected. The two terms in Equation 6.2 represent the contributions to the muon flux from pion and kaon decays respectively. The flux of $\mu^{+}$and $\mu^{-}$are generated in the ratio $\mu^{+} / \mu^{-}=1.25$ reflecting the excess of positive mesons produced by the mainly positively charged primary flux. This charge ratio is in accordance with surface measurements of low energy muons $(E<100 \mathrm{GeV})$. However it is likely to underestimate the charge ratio of muons at the Far Detector since these muons correspond to higher surface energies $(E>500 \mathrm{GeV})$ where the muon flux has a larger contribution from kaon decays [119].

In order to extrapolate the muon flux from the surface to the Far Detector, a knowledge of the density and composition of the local rock is required along with an understanding of muon energy loss through the rock. The rock above the Far Detector cavern is primarily a local type known as Lake Vermillion Greenstone, with a measured density of $2.8 \mathrm{~g} \mathrm{~cm}^{-3}[120]$. This rock is interspersed with pockets of iron ore so the average rock density varies with direction. The Far Detector cosmic muon simulation uses a rock density map calculated by the Soudan 2 experiment using cosmic muon data. This rock map is combined with a digitized map of the surface topography to find the overburden for a given direction [120]. The average muon energy loss through the rock is parameterized as follows [119]:

$$
\frac{d E}{d x}=A+\frac{E}{L}
$$

The first term on the right hand side of Equation 6.3 represents energy losses due to ionization, with $A \approx 1.9 \mathrm{MeV} / \mathrm{g} \mathrm{cm}^{-2}$. The second term represents losses due to radiative processes, with $L \approx 2.5 \times 10^{5} \mathrm{~g} \mathrm{~cm}^{-2}$. Although in practice $A$ and $L$ both 
vary slowly with energy and $d E / d x$ undergoes stochastic fluctuations, all three terms can be approximatcly treated as constant. Using this approximation, Equation 6.3 can be integrated to give the energy $E_{X}$ at depth $X$ as a function of the surface energy $E_{0}$ as follows:

$$
E_{X}=\left(E_{0}+A L\right) e^{-X / L}-A L
$$

The muon flux incident on the Far Detector is calculated as a function of energy $E_{X}$ and angle $\theta$ by propagating the surface flux through the rock overburden as follows:

$$
N\left(E_{X}, X, \cos \theta\right) d E_{X}=N\left(E_{0}, 0, \cos \theta\right) d E_{0}=N\left(E_{0}, 0, \cos \theta\right) e^{X / L} d E_{X}
$$

For each angle $\theta$, the Soudan 2 rock map is used to determine the rock overburden $X$. Equation 6.4 is then used to convert the muon energy $E_{X}$ into the equivalent surface energy $E_{0}$. Finally Equation 6.5 is used to determine the muon flux at energy $E_{X}$ and angle $\theta$ from the surface flux at energy $E_{0}$ and angle $\theta$.

\subsubsection{Data-MC Comparison of Cosmic Muon Flux}

In order to validate the Far Detector cosmic muon simulation, the simulated muon flux is compared with the muon flux measured in the data. A sample of $\mathrm{MC}$ cosmic muons is generated as follows:

1. The initial energy and direction of the muon is selected at random using the probability distribution given by Equation 6.5 .

2. The initial position of the muon is a given by a point chosen at random on the surface of a box placed around detector.

3. The muon is propagated through the detector using gminos and the detector response is simulated using PhotonTransport and DetSim.

The MC and data are compared using muon tracks that cross $>20$ planes and satisfy straight line fits with deviations of $<1 \mathrm{~cm}$ in both views. This selection provides a clean sample of well-measured muons representing the bulk of the muon spectrum. In order to perform comparisons of distributions of data and $\mathrm{MC}$, the absolute muon flux is first 


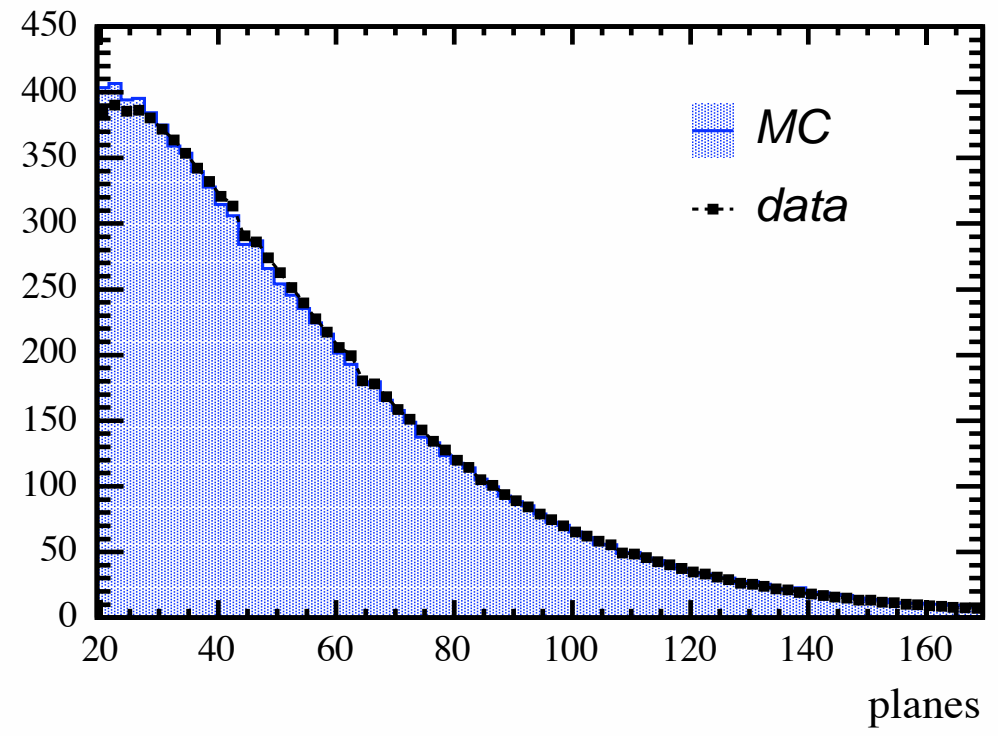

Figure 6.7: Distribution of muon track planes.

determined by normalizing the MC to the data. A sample of $1 \mathrm{M}$ simulated muons yields 337,500 selected tracks, whereas a sample of 15 days data yields 311,400 selected tracks. Assuming the selection efficiencies in data and $\mathrm{MC}$ are equal, this gives an absolute muon flux of $0.71 \mathrm{~s}^{-1}(100 \mathrm{k} \equiv 39.0 \mathrm{hrs})$. This result is in agreement with previous estimates of the absolute muon flux [72] [118] [121] [122].

Figure 6.7 shows the distribution of the total number of planes crossed by the muon tracks. There is good agreement between data and MC. Figure 6.8 shows the measured flux as a function of the reconstructed muon zenith angle. There is general agreement between data and MC. The sharp fall off in flux with zenith angle is due to the rapid increase in the rock overburden which filters out all but the highest energy muons from the surface. Figure 6.9 shows the measured flux as a function of the reconstructed muon azimuthal angle. There is general agreement between data and MC. The shape of the distribution is determined by the acceptance of the detector. For trajectories close to the $\mathrm{X}-\mathrm{Y}$ plane, there are fewer events that cross a sufficient number of planes to satisfy the selection criteria, suppressing the measured flux around the azimuthal angles of $90^{\circ}$ and $270^{\circ}$. Small differences between MC and data occur in both the zenith and azimuthal angle distributions, suggesting that systematic errors are incurred in transferring the rock density map from Soudan 2 to MINOS. 


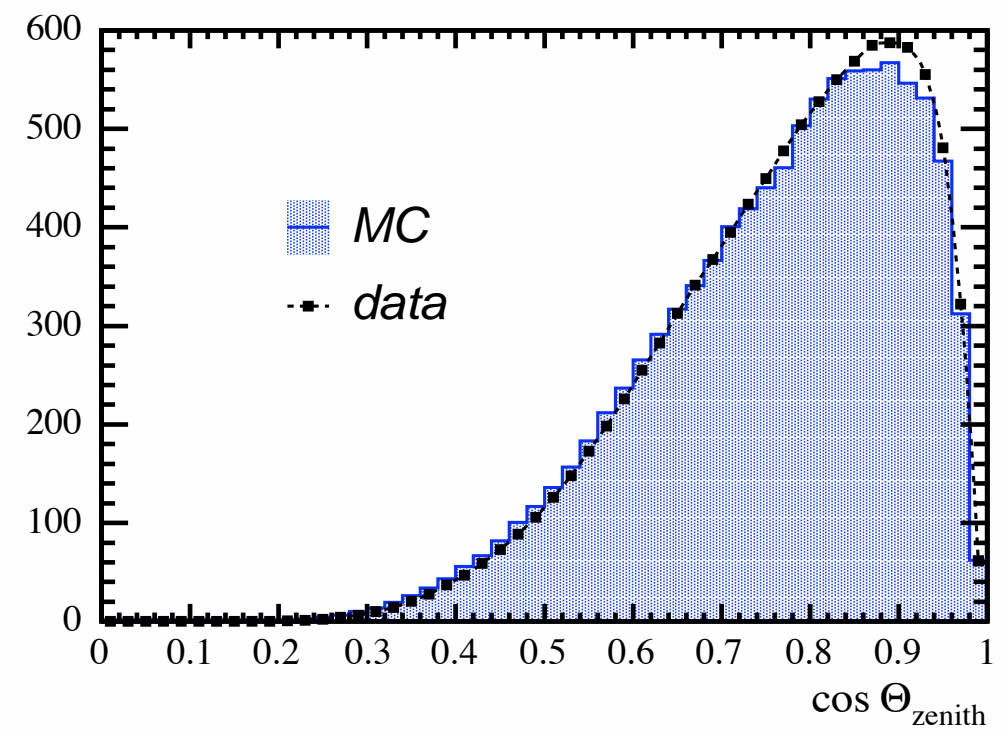

Figure 6.8: Distribution of reconstructed muon zenith angle.

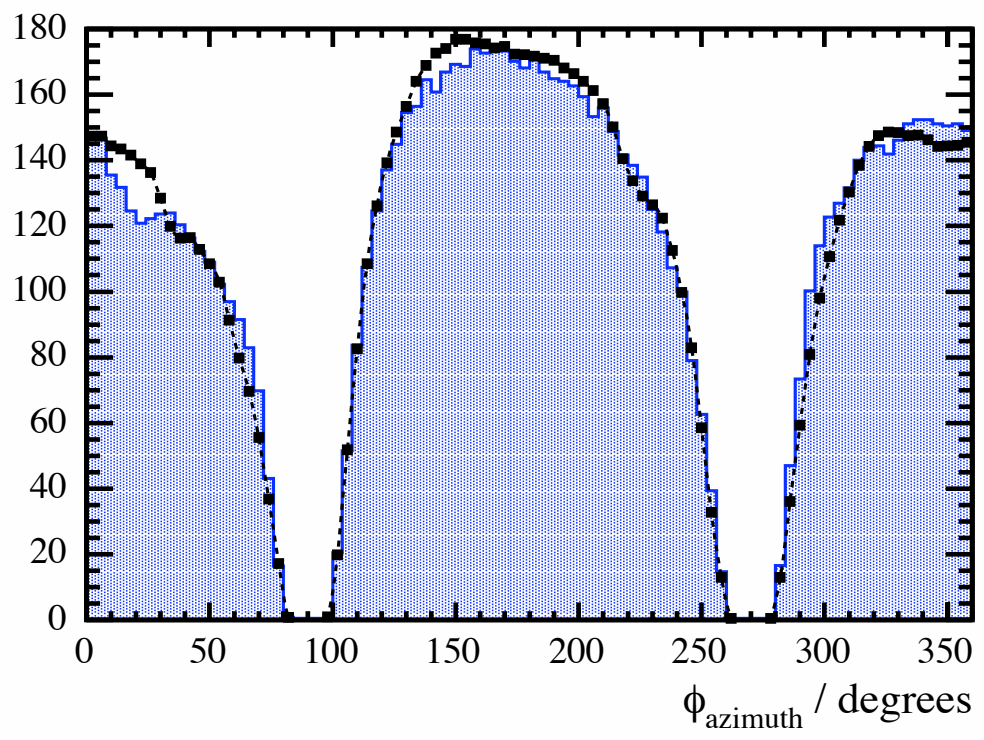

Figure 6.9: Distribution of reconstructed muon azimuthal angle. 


\subsection{Atmospheric Neutrino Simulation}

\subsubsection{Simulation of Atmospheric Neutrino Flux}

Studies of atmospheric neutrino oscillations are performed by comparing the measured flux of neutrinos in a detector with the predicted flux from simulations of cosmic ray interactions in the atmosphere. The atmospheric neutrino flux is simulated by combining a parameterization of the primary cosmic ray flux with models of neutrino yields from cosmic ray interactions in the atmosphere. A number of different neutrino flux models are available [123]. For this analysis, the flux model of Barr et al [124] is used to determine the neutrino oscillation parameters.

Barr et al simulate the atmospheric neutrino flux at the Far Detector by performing a full three-dimensional treatment of cosmic ray interactions in the atmosphere. Cosmic rays are injected at the top of the atmosphere over the whole globe, and secondary particles from cosmic ray interactions are then tracked through the atmosphere. The flux of neutrinos is measured using a detector centred at the site of the Far Detector. The details of the model are described below:

- Primary Flux: The primary cosmic ray flux is parameterized as a function of energy by applying power law fits to measurements of each type of nucleus incident on the atmosphere. Recent measurements of the dominant primary proton flux are found to agree to $5 \%$ for energies below $100 \mathrm{GeV}$, whereas measurements of the sub-dominant primary helium flux are found to agree to 15\% [123]. The cosmic ray spectrum is modulated by solar activity. During periods of high solar activity, the solar wind suppresses the low energy portion of the cosmic ray spectrum and therefore reduces the flux of low energy atmospheric neutrinos. The strength of solar activity is cyclical with a period of 11 ycars. One way of monitoring the level of solar activity over time is to measure the flux of neutrons produced by cosmic ray interactions in the atmosphere [125]. Since neutrons and neutrinos are produced with similar energies relative to the primary cosmic ray energies, they should possess a similar dependence on the level of solar activity.

The atmospheric neutrino flux at the Far Detector is simulated at solar minimum and solar maximum and neutron monitoring data is then used to interpolate between the two simulations. The atmospheric neutrino data used in this analysis 
was collected between 1st August 2003 and 31st October 2004. This came shortly after a solar maximum during 2001. The average flux of atmospheric neutrons during the period of data-taking was measured to be $5 \%$ higher than the preceding solar maximum [126], corresponding to an increase of $1 \%$ in the rate of atmospheric neutrino events above $1 \mathrm{GeV}$ relative to solar maximum [127]. Since this increase is small compared with the overall uncertainty in the absolute flux of atmospheric neutrinos, the atmospheric neutrino flux predicted for solar maximum is used for the analysis.

- Geomagnetic Fields: The magnetic field of the Earth filters the flux of cosmic rays incident on the atmosphere. The ability of a cosmic ray to penetrate through this magnetic field depends on its rigidity (rigidity $\equiv$ momentum/charge). Cosmic rays with sufficient rigidity are able to penetrate through the field and interact in the atmosphere, whereas cosmic rays with insufficient rigidity are deflected back out to space before reaching the atmosphere. The cut-off rigidity depends on the magnetic latitude and the angle at which cosmic rays approach the Earth. At high magnetic latitudes, cosmic rays are incident parallel to the lines of magnetic flux and are deflected weakly; at low magnetic latitudes, cosmic rays are incident perpendicular to the lines of magnetic flux and are deflected strongly.

Since the vast majority of cosmic rays are positively charged, the Earth's magnetic field also creates an east-west asymmetry in the cosmic ray flux. Cosmic rays arriving from the west are focused towards the Earth, whereas cosmic rays arriving from the east are defocused away from the Earth. Therefore the cut-off rigidity for cosmic rays arriving from the cast is lower than for cosmic rays arriving from the west. This effect is suppressed at higher magnetic latitudes as the focusing and defocusing starts to be directed parallel to the surface of the Earth.

The Far Detector is located at quite a high magnetic latitude $\left(56.3^{\circ}\right)$. Therefore there is a large up-down asymmetry but small east-west asymmetry in the cut-off rigidity. The average cut-off rigidity for cosmic rays incident from above is estimated to be $1 \mathrm{GcV}$, whereas the average cut-off rigidity for cosmic rays incident from below is estimated to be $10 \mathrm{GeV}$ [123]. In order to remove cosmic rays simulated below the cut-off rigidity, cosmic rays that produce one or more neutrinos in the Far Detector are traced back through the atmosphere. A trajectory is con- 
sidered valid if a particle can be propagated to a distance of $>30 R_{o}$ with a total path length of $<300 R_{o}\left(R_{o} \equiv\right.$ radius of Earth).

- Hadron Production: Cosmic rays interact in the atmosphere to produce cascades of secondary hadrons. Particle interactions in the atmosphere are generated using the Target simulation package [128]. For unstable particles, decays and interactions are competing processes. Each particle is assigned a decay and interaction length chosen at random from probability distributions. These lengths are then compared to determine whether the particle decays or interacts.

Models of hadron production have been tuned on data gathered from fixed target experiments in which protons are accelerated onto light nuclei [129] [130] [131] [132] [133]. The phase space explored by these experiments provides only limited coverage of the phase space of hadron production in the atmosphere, and the data must therefore be interpolated or extrapolated into unmeasured regions. Because of the sparse coverage of data, the uncertainties in hadron production models contribute significantly to the overall uncertainty in the atmospheric neutrino flux.

Figure 6.10 shows the angular distribution of the atmospheric neutrino flux predicted by Barr et al for a range of neutrino energies [134]. The neutrino flux falls steeply with energy, reflecting the shape of the primary spectrum. For encrgies below $1 \mathrm{GcV}$, geomagnetic effects create large up-down asymmetries in the flux. The down-going flux reflects the local geomagnetic effects, whereas the up-going flux reflects an average of the global geomagnetic effects. For energies above $1 \mathrm{GcV}$, the angular distribution becomes more up-down symmetrical, but is enhanced around the horizon. This is due to a combination of effects. Firstly, hadrons propagate for longer through the less dense high regions of the atmosphere at large zenith angles, enhancing hadronic decays relative to hadronic interactions. Secondly, high energy muons are more likely to decay before reaching the ground at large zenith angles.

The uncertainty in the overall atmospheric neutrino flux is dominated by the uncertainties in the primary flux measurements and hadron production models. The uncertainty over the neutrino energy range $1-10 \mathrm{GeV}$ is estimated to be $5 \%$ from crrors in the primary flux [134] and 10\% from errors in hadron production [135]. The uncertainty in the overall flux can also be estimated by comparing Barr et al with alternative models. These are found to differ at the level of $15 \%$ [123] [136]. 


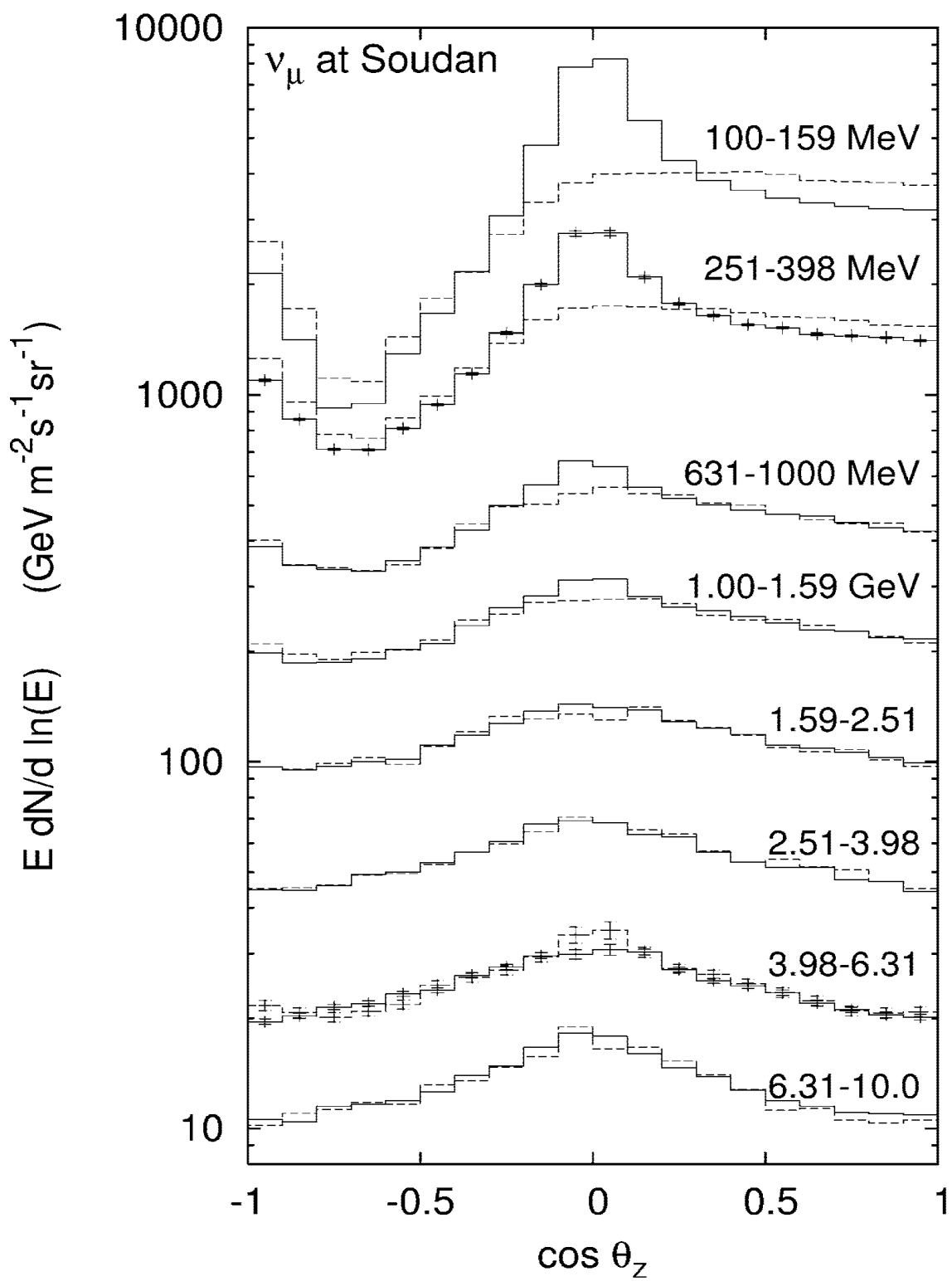

Figure 6.10: Zenith angle distribution of predicted atmospheric $\nu_{\mu}$ flux for a range of energies (solid lines $=3 D$ model, dashed lines $=1 D$ model). Figure taken from [134]. 


\subsubsection{Simulation of Atmospheric Neutrino Cross-Sections}

Neutrino interactions are simulated in the Far Detector using the Neugen 3 package [137]. This provides models for interactions between neutrinos and nucleons bound inside nuclei or quarks inside nucleons. The main interactions simulated are: Quasi-Elastic Scattering (QE) which dominates for encrgies below $1 \mathrm{GcV}$; Resonances (RES) which dominate in the energy range $1-3 \mathrm{GeV}$; and Deep Inelastic Scattering (DIS) which dominates for energies above $3 \mathrm{GeV}$. The cross-section for each type of interaction is parameterized by Neugen 3 and tuned to fit the available experimental data. For each incident neutrino, Neugen 3 determines whether an interaction will take place by comparing the total crosssection with a random number. If the result is positive, Neugen3 selects the type of interaction and simulates the hadronic final state.

For QE and RES interactions, the incident neutrino scatters off a nucleon. The bound nucleons inside a nucleus are treated as a Fermi gas. Each nucleon possesses a Formi momentum of $\simeq 230 \mathrm{McV}$ and a binding cnergy of $\simeq 30 \mathrm{McV}$. Since nuclcons are degenerate within a Fermi gas they can only be scattered into unoccupied states above the Fermi surface. Therefore neutrino interactions which produce nuclcons below the Fermi momentum are suppressed (this is known as Pauli Blocking). In QE events the neutrino scatters elastically off the nucleon. In RES events, the neutrino interacts to form a resonanee which then decays into a final state usually containing one or more pions. A total of 17 resonances are simulated by Neugen3, dominated by the production of $\Delta(1232)$. For DIS interactions, the incident neutrino scatters of a quark. The interaction cross-section is expressed in terms of structure functions calculated from the parton distribution functions. The multiplicity of the hadronic final state is then parameterized as a function of its invariant mass. Once an interaction has taken placed, the resulting hadrons may be absorbed or re-scattered as they exit the nucleus. These intra-nuclear effects are simulated by a collection of routines called Intranuke [138].

Figures 6.11 and 6.12 show the neutrino interaction cross-sections on ${ }^{56} \mathrm{Fe}$ nuclei simulated by Neugen 3 as a function of the neutrino energy for $\nu_{\mu}$ and $\bar{\nu}_{\mu}$ charged current interactions. The total cross-section is plotted, along with the exclusive QE, RES and DIS cross-sections. For multi-GeV energies where the majority of atmospheric neutrino events are observed in the Far Detector, all three types of interaction contribute significantly to the overall cross-section. The uncertainty in the atmospheric neutrino event rate due to errors in the interaction model is estimated to be $10 \%$ [139]. 


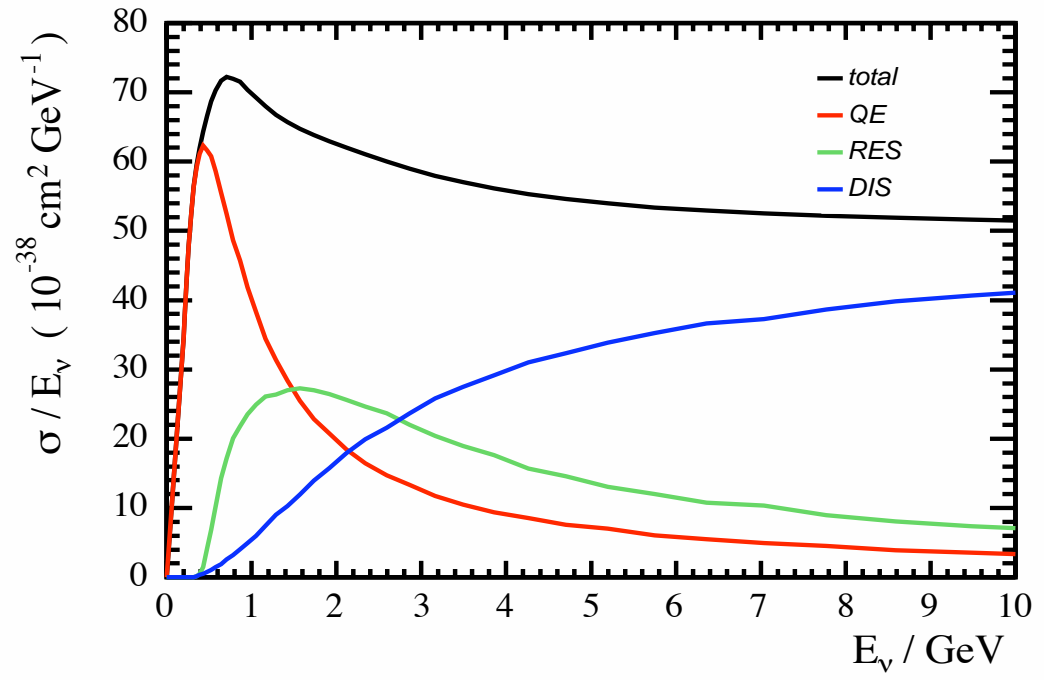

Figure 6.11: Cross-section for $\nu_{\mu} C C$ interactions as a function of neutrino energy, generated using the Neugen3 model of neutrino interactions.

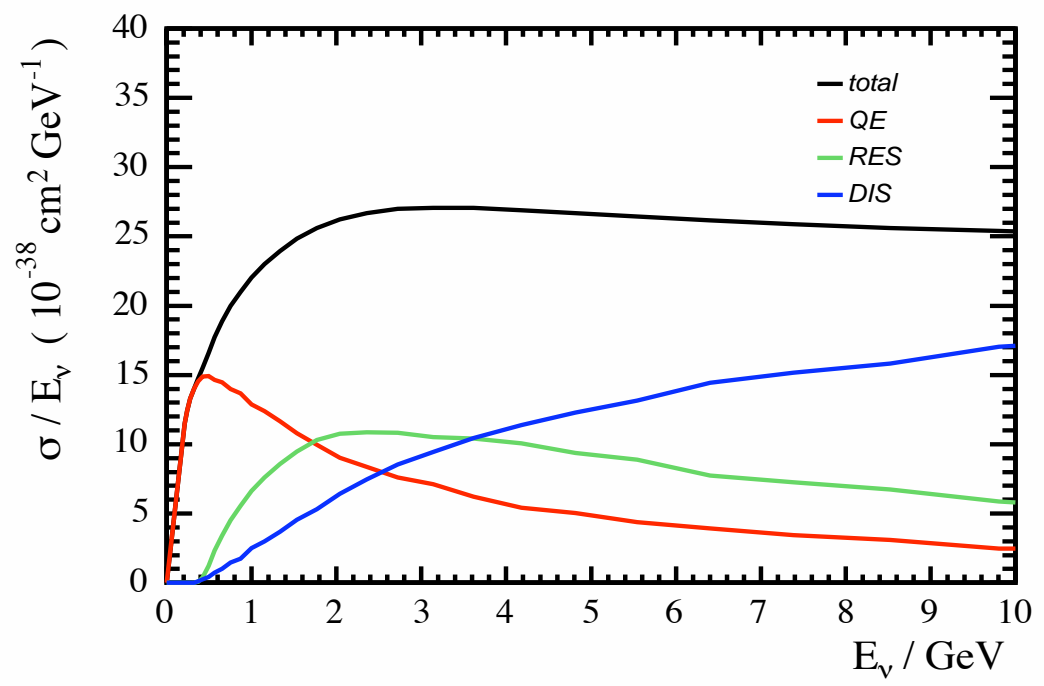

Figure 6.12: Cross-section for $\bar{\nu}_{\mu} C C$ interactions as a function of neutrino energy, generated using the Neugen3 model of neutrino interactions. 


\subsection{Monte Carlo Samples}

The atmospheric neutrino analysis is developed and tuned using large MC samples of atmospheric neutrino signal and cosmic muon background. Systematic effects are studied by replacing the components of the default simulation with alternative packages. Table 6.1 gives a full list of all MC samples used in the analysis.

The central atmospheric neutrino MC samples use the flux model of Barr et al and interaction model of Neugen3. The propagation of hadrons is simulated using the GCALOR package [140] [141] which has been found to give a good description of low energy hadronic interactions in the MINOS detectors [96]. In order to estimate systematic crrors arising from the simulation, additional MC samples are generated using the flux model of Battistoni et al [142] in place of Barr et al, and the GHEISHA model of hadronic interactions [143] in place of GCALOR.

A large sample of $19 \mathrm{M}$ cosmic muons is used to study the background in the selection

of atmospheric neutrino events. A special sample of cosmic muons with energies below 2 $\mathrm{GeV}$ is also used to study the backgrounds in the selection of up-going neutrino events. Finally, additional MC samples are generated to estimate smaller backgrounds arising from neutrons emitted by muon spallation in the rock [144], neutrino-induced upwardgoing muons [118], and tau neutrinos produced by $\nu_{\mu} \rightarrow \nu_{\tau}$ oscillations. 


\begin{tabular}{|c|c|c|}
\hline MC Sample & Events & Exposure \\
\hline atmospheric neutrinos & & \\
atmos $\nu_{e} / \nu_{\mu}$ (Barr et al, Neugen 3, GCALOR) & $351 \mathrm{k}$ & $1260 \mathrm{kT}$-Yrs \\
atmos $\nu_{e} / \nu_{\mu}$ (Barr et al, Neugen 3, GHEISHA) & $100 \mathrm{k}$ & $361 \mathrm{kT}$-Yrs \\
atmos $\nu_{e} / \nu_{\mu}$ (Battistoni et al, Neugen 3, GCALOR) & $74.2 \mathrm{k}$ & $257 \mathrm{kT}$-Yrs \\
atmos $\nu_{\tau}$ (Barr et al, Neugen 3, GCALOR) & $71.3 \mathrm{k}$ & $370 \mathrm{kT}$-Yrs \\
\hline cosmic muons & & \\
cosmic $\mu$ (full spcctrum) & $18.6 \mathrm{M}$ & 0.827 Yrs \\
cosmic $\mu$ (<2 GeV) & $2 \mathrm{M}$ & $4.76 \mathrm{Yrs}$ \\
\hline other backgrounds & $47.5 \mathrm{k}$ & $200 \mathrm{Yr} s$ \\
$\nu$-induced muons $[118]$ & $45.8 \mathrm{k}$ & $2.79 \mathrm{Yr} s$ \\
\hline$\mu$-induced neutrons $[144]$ & &
\end{tabular}

Table 6.1: MC samples generated for atmospheric neutrino analysis. The physics models used to generate each sample is listed along with the number of generated events and the equivalent detector exposure. 


\section{Chapter 7}

\section{Event Reconstruction}

\subsection{Reconstruction Overview}

The particle tracks and showers in each event are reconstructed from the hits recorded in the detector. These reconstructed tracks and showers are used to identify the type of event and determine its kinematics. In order to optimize the measurement of atmospheric neutrino oscillations, a clean and well reconstructed sample of charged current $\nu_{\mu}$ and $\bar{\nu}_{\mu}$ events must be separated from the data. The signature of an atmospheric $\nu_{\mu} / \bar{\nu}_{\mu}$ CC interaction in the Far Detector is a muon track with a contained interaction vertex. The muon track may also be accompanied by a hadronic shower at the interaction vertex. Particle tracks and showers are reconstructed by identifying characteristic topologies in events. The reconstruction of atmospheric neutrino events in the Far Detector presents a number of specific challenges:

- Detector Geometry : The Far Detector is optimized for the detection and measurement of neutrino interactions from the NuMI beam. The beam neutrinos enter the detector along the positive Z-axis and interact to produce tracks and showers directed along mainly horizontal trajectories. The detector planes are aligned vertically to maximize the sampling of these tracks and showers, and the steel planes are magnetized toroidally to focus muons produced in $\nu_{\mu} \mathrm{CC}$ intcractions. In contrast to beam neutrinos, the flux of atmospheric neutrinos is roughly isotropic. This means that the sampling and focusing of events is less optimal, degrading the performance of the reconstruction. 
- Direction and Charge Reconstruction : Beam neutrino interactions produce negatively charged muons directed close to the positive Z-axis. In contrast, the flux of atmospheric neutrinos contains both $\nu_{\mu}$ and $\bar{\nu}_{\mu}$ neutrinos and is incident on the detector from all directions. Therefore both the direction and charge of muons produced by atmospheric neutrino interactions must be reconstructed on an eventby-event basis. The muon direction is determined by fitting the measured times along the track, and the muon charge is determined by measuring the curvature of the track in the magnetic field.

- Cosmic Muon Background : The vertical alignment of detector planes presents a particular problem in separating the atmospheric neutrino signal from the cosmic muon background. The angular distribution of cosmic muons is peaked towards vertically down-going events. Cosmic muons entering the detector between two detector planes can penetrate deep into the detector and appear as contained events. The cosmic muon background must be reduced by a factor of $>10^{6}$ in order to separate the atmospheric neutrino signal cleanly. The event reconstruction must be accurate and robust in order to achieve this high level of background rejection.

An extensive software library has been developed for the reconstruction of physics events in the Far Detector. The events are initially processed at the raw hit level. Each detector hit contains an eight-fold ambiguity resulting from the multiplexing of fibres into pixels. The event must be de-multiplexed to determine which strips have been hit in the event. This is done by identifying common strips from the possibilities on each side of the detector. Each reconstructed strip provides a 2D measurement of an event in either the $\mathrm{L}$ or $\mathrm{V}$ view. A pattern recognition algorithm is applied to the strips in each event to reconstruct particle tracks and showers. The algorithm first identifies $2 \mathrm{D}$ tracks and $2 \mathrm{D}$ showers in each vicw and then combines the two vicws to form $3 \mathrm{D}$ tracks and 3D showers. Once the tracks and showers in each event have been identified, they are combined to form physics events.

The software used in this analysis has been developed specifically for the reconstruction of atmospheric neutrino events in the Far Detector. The software is designed to identify the characteristic topologies of both signal and background events and to provide robust reconstruction in a high background environment. 


\subsection{Low Level Reconstruction}

The initial stage of the reconstruction is to process events at the raw hit level. The raw hits in each event are first un-packed and converted into digits. Each digit contains a digital measurement of timing and pulse height made in a VA channcl, and a list of the eight strips that are coupled to the corresponding PMT pixel. The digits form the primary input to the reconstruction chain. Digits resulting from instrumental noise or cross-talk are first identified and removed. The remaining hits are then de-multiplexed to determine the strips that make up the event.

Most events contain one or more digits resulting from single photon noise on the PVT photo-cathodes or natural radioactivity in the detector. In regions of the detector located away from the main body of an event, the typical noise rate is $1 \mathrm{kHz}$ per plane. This rate is restricted by the $2 / 36$ trigger which requires one or more coincident hits within a VARC to activate the readout. Within the event region itsclf the $2 / 36$ trigger is satisfied by the digits in the event and the noise rate rises to $>10 \mathrm{kHz}$ per plane. Since the $4 / 5$ trigger retains all digits recorded in a window $<150 \mathrm{~ns}$ around the beginning and end of the event, there is a good chance that an event will contain one or more digits resulting from noise. The noise-induced activity is filtered from each event by removing digits displaced from the main body of the event in space and time. The main body of digits that has satisfied the $4 / 5$ plane trigger is first identified. This contains all the digits in the regions where 4 out of 5 contiguous planes are occupied. Digits are then rejected if they are displaced from this body of digits by $>10$ planes of if they occur $>50$ ns before or $>500$ ns after its earliest digit.

Cross-talk between neighbouring readout channels generates additional hits in the detector adjacent to genuine physics hits. Cross-talk arises from leakage of light between neighbouring pixels on a PMT photo-cathode (optical cross-talk) or from leakage of charge between neighbouring channels on a PMT photo-anode (electrical cross-talk). Cross-talk is characterized by digits of low pulse height adjacent to digits of much larger pulse height. The degree of cross-talk between two pixels depends on their relative position on the P.MT. Typical fractions of pulse height that leak between pixcls are $1 \%$ for directly adjacent pixels and $0.1 \%$ for diagonally adjacent pixels. Cross-talk digits are tagged by analysing the pulse height and the relative position of the digits on each P.TT. Thresholds are applied to the absolute pulse height of all digits and the relative 
pulse height of adjacent digits within a PMT. Once the cross-talk in an event has been tagged, the cross-talk charge is added back into the genuine physies hits.

The remaining digits are de-multiplexed to determine which strips have been hit in the event. The cight-fold ambiguities arising from the multiplexing of fibres into pixels are resolved by comparing the possible strips on each side of the detector. The multiplexing of fibres into pixels is arranged differently on each side of the detector. In each plane, the 192 strips are divided into 8 groups of 24 consecutive strips. The groups are connected in turn to 24 pixels in $1 \frac{1}{2}$ PMTs on each side of the detector. On the west side of the detector, each group of 24 strips is connected to the 24 pixels in the same sequence; on the east side of the detector, the first group of strips is connected to the pixels in the same sequence, and the sequence is then permuted by one pixel for each subsequent group of strips. This multiplexing pattern ensures that each strip has a unique cast-west combination of pixcls and that strips sharing a common pixel are separated within each plane by more than 23 strips.

Events are de-multiplexed using the AltDeMux [145] algorithm which has becn developed for the atmospheric neutrino analysis. AltDeMux first uses the digits on each side of the detector to produce a list of all the possible solutions in each plane. The measured times of digits on each side of the detector are used to constrain the event region in each plane and reduce the number of possible solutions. To de-multiplex the event, AltDeMux forms structures of strips that are reconstructed unambiguously within a plane. These are formed from configurations with a unique solution, or a unique solution which places strips close together in a plane. The structures are used as a template to de-multiplex the remaining digits. A provisional event type is determined for each event and used to steer the de-multiplexing strategy.

Figure 7.1 shows an example of a $\nu_{\mu} \mathrm{CC}$ event generated by the MC simulation before and after de-multiplexing. The left pand shows the strips corresponding to each digit in the event. The event can be located in eight separate regions on each side of the detector. There is significant overlap between one pair of regions. AltDeMux de-multiplexes the digits that have unambiguous solutions and uses these strips to form the basic structure of the event. The remaining digits are then de-multiplexed around this structure. The right panel shows the final de-multiplexed event. The AltDeMux algorithm has been found to correctly reconstruct $98.8 \%$ of charge in cosmic muon events and $98.3 \%$ of charge in atmospheric neutrino events [145]. 

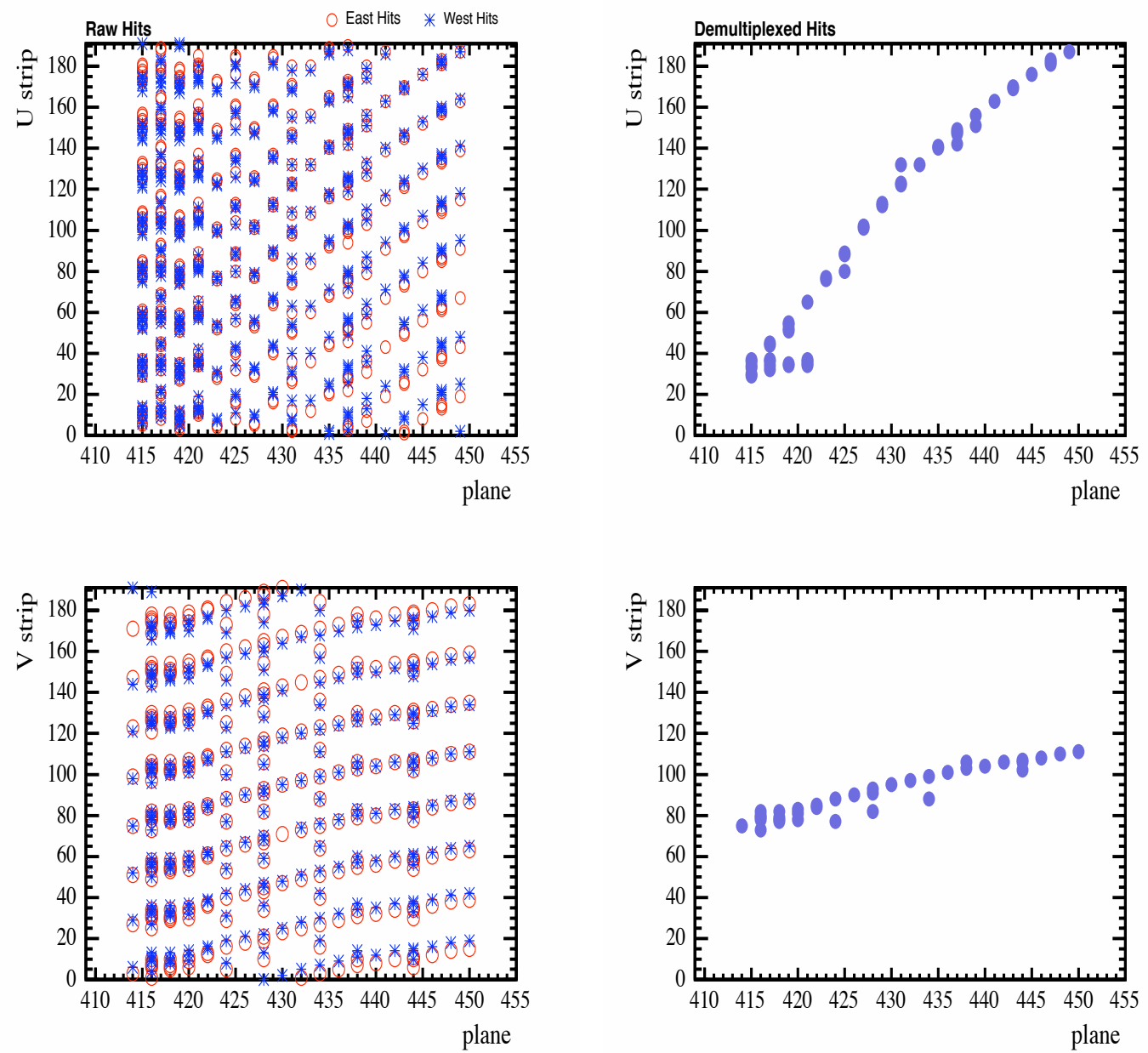

Figure 7.1: An example of a simulated $\nu_{\mu} C C$ event before and after demultiplexing. The left panel shows the eight possible strips corresponding to each digit in the event. The red circles indicate digits on the east side of the detector, and the blue stars indicate digits on the west side of the detector. The possible solutions on each side of the detector correspond to eight separate regions within the detector. There is significant overlap between one pair of these regions. This forms the basis of the de-multiplexed solution. The right panel shows the final de-multiplexed event. 


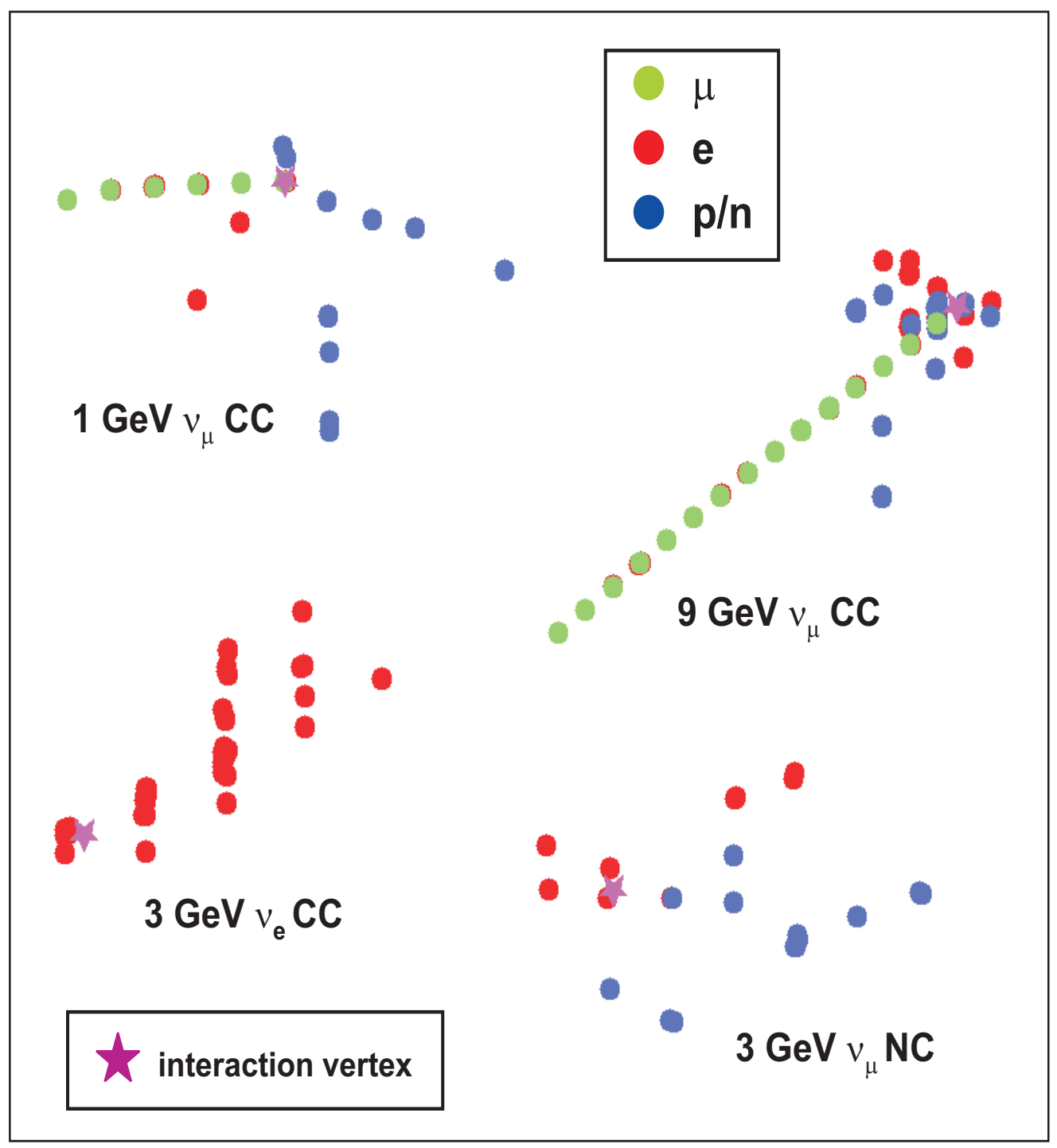

Figure 7.2: Some examples of atmospheric neutrino interactions generated in the Far Detector by the MC simulation. The detector hits are colourcoded according to particle type. The type of interaction can be identified by the event topology: $\nu_{\mu} C C$ interactions are identified by muon tracks; $\nu_{e} C C$ interactions are identified by electromagnetic showers; and $\mathrm{NC}$ interactions are identified by hadronic showers. 


\subsection{Reconstruction of Particle Tracks and Showers}

Particle tracks and showers are reconstructed by analysing the topology of events. Pattern recognition algorithms are used to identify characteristic topologies in each event. Figure 7.2 shows some examples of atmospheric neutrino interactions generated in the Far Detector by the MC simulation. The detector hits are colour-coded according to particle type. Different types of particles produce different patterns of hits in the detector. Minimally ionizing particles with sufficient encrgy to cross several planes produce track-like topologies in the detector. Low energy particles grouped together in hadronic or electromagnetic showers produce shower-like topologies in the detector. The type of neutrino interaction is identified using the event topology: $\nu_{\mu}$ CC interactions are identified by muon tracks; $\nu_{e} \mathrm{CC}$ interactions are identified by electromagnetic showers; and NC interactions are identified by hadronic showers.

For this analysis, the event reconstruction is performed using the AtNuReco algorithm, which has been developed for the atmospheric neutrino analysis. AtNuReco reconstructs tracks and showers by identifying the characteristic topologies in each event. The $\mathrm{U}$ and $\mathrm{V}$ vicws are initially treated scparately. Groups of strips with track-like and shower-like topologies are identified and used to reconstruct 2D tracks and 2D showers. The two views are then combined to form 3D tracks and $3 \mathrm{D}$ showers. The algorithm is summarized below (see Figure 7.3):

- 1D Clusters : The adjacent strips in each plane are first joined together to form $1 \mathrm{D}$ clusters. These clusters are used to identify track-like and shower-like topologies within the event. Track-like topologies are identified as groups of single strips that form continuous lines in adjacent planes. Shower-like topologies are identified as groups of multiple strips that are clustered together in adjacent planes.

- 2D Tracks : 2D tracks are reconstructed by joining together associated groups of track-like clusters in cach vicw. The clusters are initially analysed in groups of three adjacent planes. If a group of adjacent clusters forms a continuous line, the clusters are joined together to form a track segment. Segments formed from clusters containing isolated strips are tagged as track-like. Adjacent segments are analysed to identify possible joins between segments. Associations are formed between adjacent segments which contain common clusters and point in a common direction. Segments that are unambiguously associated with each other are 


\section{Track and Shower Reconstruction}

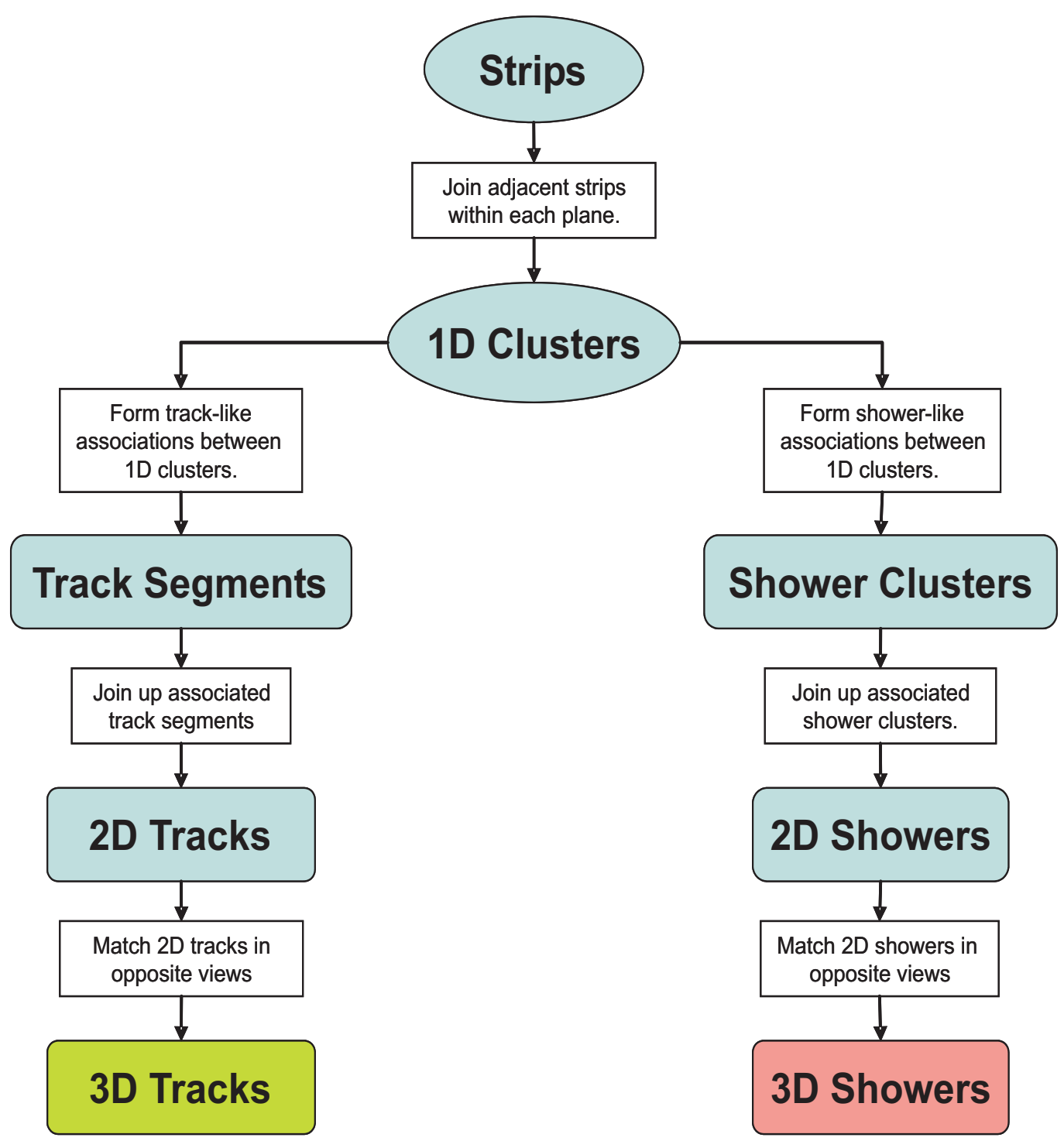

Figure 7.3: Track and shower reconstruction algorithm. 
initially joined together. Additional joins are then made by favouring the associations between longer segments. The segments that have been tagged as track-like are used to seed 2D tracks. A list of candidate 2D tracks is formed by considering all possible joins between these seed segments and other segments. One of these candidates is then selected to become a $2 \mathrm{D}$ track using an algorithm based on the length and smoothness of the candidate tracks. Any track-like segments that are not already part of $2 \mathrm{D}$ tracks are used to seed further $2 \mathrm{D}$ tracks until no more track-like segments are left.

- 2D Showers : 2D showers are reconstructed by joining together associated groups of shower-like clusters in each vicw. The clusters are initially analysed in groups of three adjacent planes. Two sizes of clustering window are placed around each cluster. The first window is placed level with the top and bottom of the cluster. If the window contains $\geq 2$ clusters and $\geq 5$ strips, the cluster is tagged as being shower-like. This window is used to identify dense showers, typically electromagnetic in origin. The second window is placed \pm 2 strips from the top and bottom of cluster. If the window contains $\geq 4$ clusters and $\geq 4$ strips, the cluster is tagged as shower-like. This window is used to identify diffuse showers, typically hadronic in origin. In addition, clusters containing a total pulse height of $>50$ PEs are tagged as shower-like. The clusters that have been tagged as shower-like are used to seed $2 \mathrm{D}$ showers. A window of \pm 4 planes and \pm 5 strips is placed around each cluster in the shower. Any clusters inside this window that are shower-like or not track-like are added to the shower. This clustering continues until no more clusters can be added to the shower. Any shower-like clusters that are not already part of 2D showers are then used to seed further 2D showers until no more shower-like clusters are left.

- 3D Tracks : 3D tracks are formed by joining together pairs of matching 2D tracks in opposite views. Pairs of 2D tracks are required to overlap and be separated by $<10$ planes at each end in order to be joined together. If multiple 2D tracks are paired together, the closest matched pairs are joined together. Once a 3D track has been formed, the strips within each of its clusters are sorted to select the strips associated with the track. For clusters tagged as track-like, all the strips are added to the track. For clusters not tagged as track-like, linear fits are applied to the 
surrounding track-like clusters and the strips closest to these fits are added to the track. Any strips found to be outlying from the fits are dropped from the track. Only $3 \mathrm{D}$ tracks occupying $\geq 6$ planes are retained.

- 3D Showers : 3D showers are formed by joining together pairs of matching 2D showers in opposite vicws. The pairs of $2 \mathrm{D}$ showers are required to overlap in order to be joined together. If multiple 2D showers are paired together, the closest matched pairs are joined together. Once existing 2D showers have been joined together, the ends of each 3D track are 11sed to seed new showers. If a track vertex is not associated with an existing 3D shower, the strips around the track vertex are clustered together to form a $3 \mathrm{D}$ shower. If no 3D shower or $3 \mathrm{D}$ track has been formed, an additional phase of shower formation is initiated. A much looser clustering algorithm is applied to the event, requiring $\geq 4$ strips within a window of 9 planes with at least one strip in each view. In the final stage of shower reconstruction, any strips in the event that are not part of a track or shower are added to the nearest shower within a window of \pm 16 planes and \pm 24 strips.

The performance of the AtNuReco reconstruction is tested using a sample of MC atmospheric $\nu_{\mu} / \bar{\nu}_{\mu}$ CC cvents (sec Section 6.5). The event sample is required to satisfy the following conditions:

1. The neutrino interaction vertex is required to be $>0.5 \mathrm{~m}$ from the edges of the detector and $>5$ planes from the ends of each supermodule.

2. The muon is required to cross $\geq 6$ planes, corresponding to the minimum number of planes for a track to be reconstructed.

Figure 7.4 shows an example of a reconstructed $\nu_{\mu} / \bar{\nu}_{\mu} \mathrm{CC}$ event from this sample. The signature used to identify $\nu_{\mu} / \bar{\nu}_{\mu}$ CC events is a reconstructed muon track. The track reconstruction must be accurate and efficient in order to sclect a clean sample of events. Figure 7.5 shows the efficiency as a function of the number of planes crossed by the muon. The efficiency rises with the number of planes as the muon track becomes clearcr, and approaches $100 \%$ above 20 planes. Figure 7.6 shows the efficiency as a function of the muon energy. The mean energy required for a muon to cross 6 planes is $300 \mathrm{MeV}$. The efficiency rises sharply above this threshold as the mean number of planes crossed by the muon increases, and then levels off at $95 \%$ above $1 \mathrm{GeV}$. The levelling off 

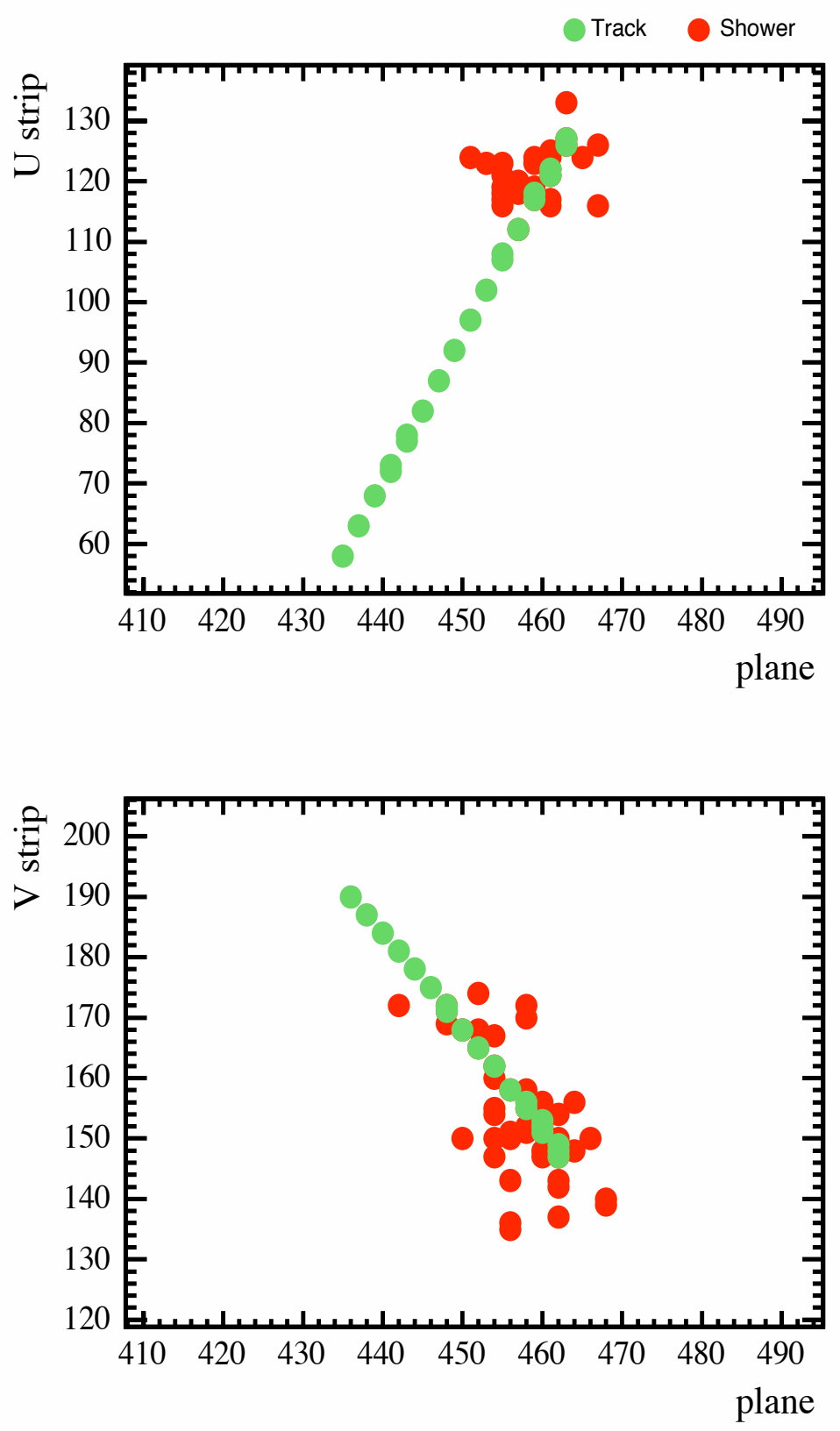

Figure 7.4: Example of tracks and showers reconstructed in a atmospheric $\nu_{\mu} C C$ interaction generated in the Far Detector by the MC simulation. The green circles indicate strips assigned to the track, the red circles indicate strips assigned to the shower. 
in the efficiency below $100 \%$ is due to high energy muons which cross a small number of planes before exiting the detector. The overall efficiency for reconstructing muon tracks in the event sample is calculated to be $91.4 \%$.

In order to select a pure sample of $\nu_{\mu} / \bar{\nu}_{\mu} \mathrm{CC}$ events, the efficiency for reconstructing tracks in $\nu_{e} / \bar{\nu}_{e} \mathrm{CC}$ and $\mathrm{NC}$ events must be low. This is tested using a sample of $\mathrm{MC}$ atmospheric $\nu_{e} / \bar{\nu}_{e} \mathrm{CC}$ and $\mathrm{NC}$ events. The events are required to span $\geq 6$ planes and satisfy the same containment conditions as the $\nu_{\mu} / \bar{\nu}_{\mu}$ CC sample. The track reconstruction efficiency is calculated to be $13.8 \%$ for $\nu_{e} / \bar{\nu}_{e}$ CC events and $25.6 \%$ for $\mathrm{NC}$ events. The majority of the reconstructed tracks are shorter than 10 planes and tag either a pion or a continuous group of strips within the event.

The quality of the muon track reconstruction is measured by comparing the strips assigned to each muon track with the true muon strips in each event. The following two measures of track quality are used:

$$
\begin{aligned}
& \text { track efficiency }=\frac{\# \text { true muon strips on reconstructed track }}{\# \text { true muon strips }} \\
& \text { track purity }=\frac{\# \text { reconstructed strips on true muon track }}{\# \text { reconstructed strips }}
\end{aligned}
$$

The track efficiency represents the percentage of true muon strips that form part of the reconstructed track. The track purity represents the percentage of strips on the reconstructed track that form part of the true muon track. These quantities measure the combined accuracy of the de-multiplexing and track-finding.

Figure 7.7(a) shows the distribution of track efficiencies for reconstructed tracks in the $\nu_{\mu} / \bar{\nu}_{\mu}$ CC event sample. Figure 7.7(b) shows the mean efficiency as a function of track planes. The mean efficiency is higher than $90 \%$ for all tracks that cross $\geq 6$ planes. The overall efficiency is calculated to be $94.8 \%$. Figure 7.8(a) shows the distribution of track purities for reconstructed tracks in the $\nu_{\mu} / \bar{\nu}_{\mu}$ CC event sample. Figure 7.8(b) shows the mean purity as a function of track planes. The mean purity is higher than $90 \%$ for all tracks that cross $\geq 6$ planes. The overall purity is calculated to be $95.4 \%$. Both the efficiency and purity are improved by the track quality cuts applied to separate the atmospheric neutrino signal from the cosmic muon background. 


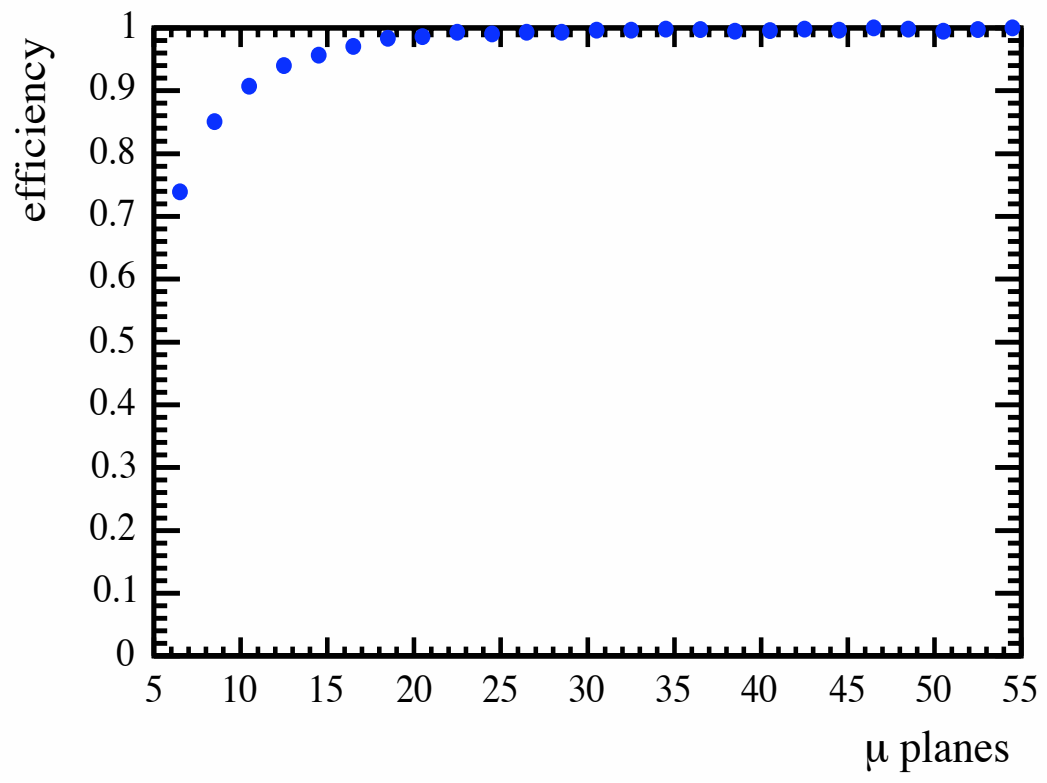

Figure 7.5: Efficiency for reconstructing muon tracks in $M C$ atmospheric $\nu_{\mu} / \bar{\nu}_{\mu} C C$ events as a function of planes crossed by the muon.

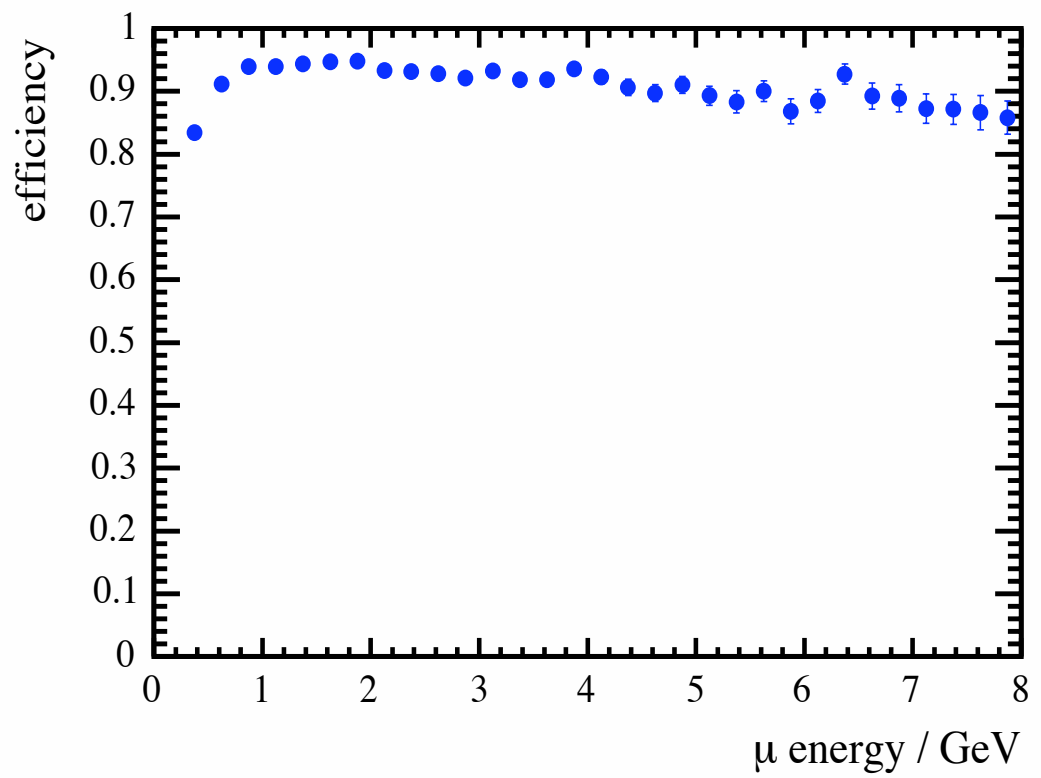

Figure 7.6: Efficiency for reconstructing muon tracks in $M C$ atmospheric $\nu_{\mu} / \bar{\nu}_{\mu}$ CC events as a function of the muon energy. 

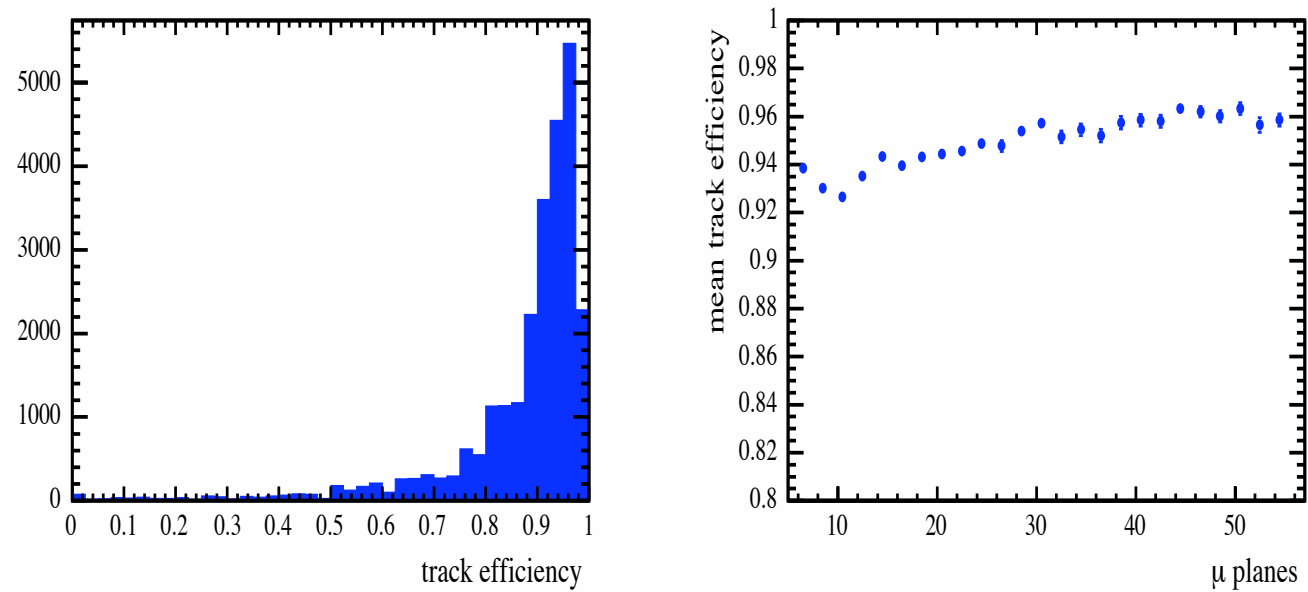

Figure 7.7: (a) distribution of track efficiencies for reconstructed muon tracks in $M C$ atmospheric $\nu_{\mu} / \bar{\nu}_{\mu} C C$ events (b) mean track efficiency as a function of the number of muon track planes.
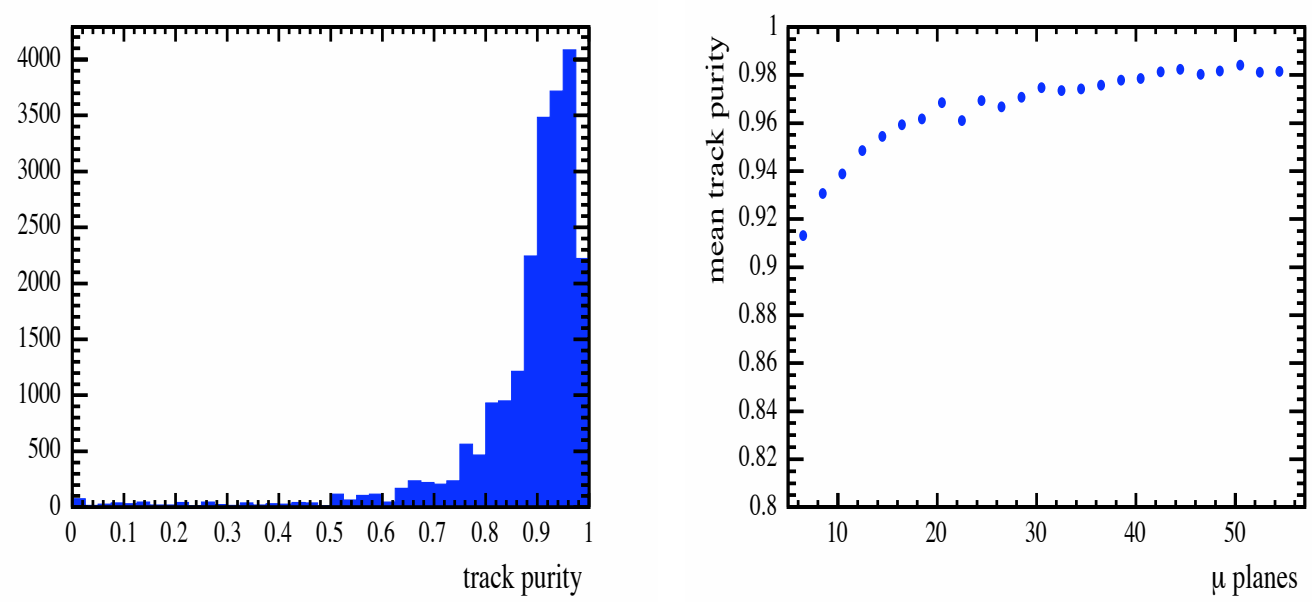

Figure 7.8: (a) distribution of track purities for reconstructed muon tracks in $M C$ atmospheric $\nu_{\mu} / \bar{\nu}_{\mu} C C$ events (b) mean track purity as a function of the number of muon track planes. 


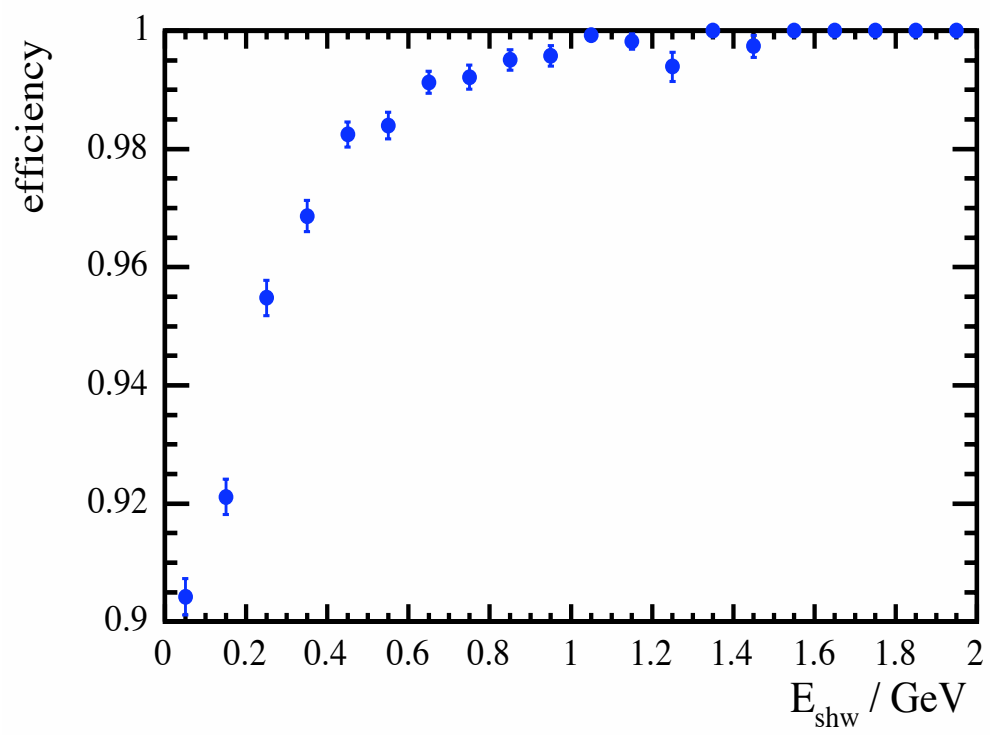

Figure 7.9: Efficiency for reconstructing vertex showers in $M C$ atmospheric $\nu_{\mu} / \bar{\nu}_{\mu} C C$ events as a function of the kinetic energy of the showers.

The majority of atmospheric $\nu_{\mu} / \bar{\nu}_{\mu} \mathrm{CC}$ events contain visible shower energy at the interaction vertex. The vertex shower typically carries away: $15 \%$ of energy in quasielastic interactions, $30 \%$ of energy in resonance interactions, and $50 \%$ of energy in deep inelastic interactions. The overall neutrino energy is determined by summing the muon momentum with the shower energy. It is important to reconstruct showers efficiently in order to obtain an accurate measurement of the neutrino energy. The showers typically deposit energy in $10-20$ strips per GeV. Therefore low energy showers may contain only a small number of strips. If a shower has not already been reconstructed at the track vertex, a loose clustering algorithm is applied to detect any shower activity. All strips around the track vertex that are not part of the reconstructed track are clustered together to form a shower. At low energies, vertex showers may not be visible if the shower particles fall below the detector threshold, sneak between two planes, get absorbed by the steel, or overlap with muon track. Figure 7.9 shows the efficiency for reconstructing vertex showers as a function of the true kinetic energy of the showers for $\nu_{\mu} / \bar{\nu}_{\mu}$ CC events containing a reconstructed track. Since the conditions for reconstructing a vertex shower are extremely loose, the efficiency remains above $90 \%$ over the entire range of energies and approaches $100 \%$ for energies above $1 \mathrm{GeV}$. 


\subsection{Reconstruction of Event Kinematics}

\subsubsection{Direction Reconstruction}

The propagation direction is important for the selection of atmospheric neutrino events and the measurement of atmospheric neutrino oscillations. Up-going events provide a signature for atmospheric neutrino events, and a deficit in the measured rate of up-going events provides a signature for atmospheric neutrino oscillations. For this analysis, the propagation direction of the neutrino is approximated by the propagation direction of the reconstructed muon track.

The muon propagation direction is determined using timing information. The measured times of the hits along the track are corrected to account for calibration offsets and propagation times along the readout fibres. The corrected times are then compared with the distances of the hits along the track. Two charge-wcighted lincar fits are applied with fixed gradients $\beta= \pm 1$, coresponding to the muon travelling forwards and backwards along the track at the speed of light. The $r m s$ deviation of each fit is calculated. Any outlying hits displaced from a fit by more than the rms deviation plus 10 ns are removed from the fit and the rms deviation is re-calculated. The reconstructed direction is given by the fit with the smallest rms deviation. The difference between the rms deviations ( $\Delta \mathrm{rms}$ ) is used to measure the quality of the direction determination and separate clean samples of up-going and down-going events.

The performance of the direction reconstruction is tested using stopping cosmic muons. Since cosmic muons are entirely down-going, they provide a source of particles with a common direction that can be used to calculate the efficiency for correctly reconstructing the direction. This is defined as the proportion of stopping muons that are reconstructed as down-going. Stopping muons are chosen because they reflect the cnergy and topology of the atmospherie neutrino signal better than the through-going muons that make up the majority of cosmic muon events. A clean sample of stopping muons is obtained from the data and $\mathrm{MC}$ by selecting events with reconstructed tracks that satisfy the following containment and quality cuts:

1. The upper vertex is required to be $<0.5 \mathrm{~m}$ or $\leq 5$ planes from the detector edges: the lower vertex is required to be $>0.5 \mathrm{~m}$ and $>5$ planes from detector edges.

2. Tracks are required to cross $\geq 8$ scintillator planes. 
3. Tracks are required to contain $\geq 5$ track-like planes (defined as those planes for which the total pulse height is $<80$ PEs and $>80 \%$ of the total pulse height is within \pm 1 strip of the track position in the plane).

4. Tracks are required to contain $>50 \%$ of the total pulse height in the event.

Timing fits are applied to the muon tracks assuming first that the muon is travelling downwards at the speed of light and then that the muon is travelling upwards at the specd of light. The rms deviations for the down-going ( $\left.r m s_{d o w n}\right)$ and 11p-going $\left(r m s_{u p}\right)$ timing fits are calculated and the muon direction is given by the fit with the smallest rms deviation. Figure 7.10 shows some examples of timing fits for down-going stopping muons and 11p-going neutrino-induced muons sclected from the data.

Figure 7.11 shows the distribution of rms deviations for down-going timing fits to stopping muons in data and MC. The mean rms deviation is calculated to be $2.49 \pm$ $0.04 \mathrm{~ns}$ in data and $2.44 \pm 0.04 \mathrm{~ns}$ in MC. There is a $2 \%$ difference between data and MC. This arises from the small discrepancies in the $\mathrm{MC}$ simulation. The Gaussian smearing technique used to tune the timing simulation is an approximation which starts to break down for short tracks where the direction is determined using only a small number of timing measurements. Figure 7.12 shows the distribution of $r m s_{u p}-r m s_{d o w n}$ for data and MC. The majority of events occur in the region $r m s_{u p}-r m s_{d o w n}>0$ and the distributions fall steeply as the value of $r m s_{u p}-r m s_{d o w n}$ becomes negative. The overall reconstruction efficiency is calculated to be $99.5 \%$ in data and $99.6 \%$ in MC. Figure 7.13 shows the reconstruction efficiency as a function of the number of track planes for data and MC. The efficiency rises above $90 \%$ for tracks that cross $\geq 8$ planes, with good agreement between data and MC for tracks that cross $\geq 10$ planes. Figure 7.14 shows the reconstruction efficiency as a function of $\Delta r m s$. The efficiency rises from $50 \%$ at $\Delta r m s=0$, where the muon direction is ambiguous, to $>95 \%$ for $\Delta r m s>0.5$. There is good agrecment between data and $\mathrm{MC}$ over the cntire range of $\Delta r m s$ values.

The performance of the direction reconstruction in atmospheric neutrinos is tested using the $\mathrm{MC}$ atmospheric $\nu_{\mu} / \bar{\nu}_{\mu}$ CC event sample described in Section 7.3. The reconstructed muon tracks are required to satisfy the track quality cuts applied to stopping muons. The $\geq 8$ track plane cut removes $13 \%$ of the events, while the $\geq 5$ track-like plane cut and $>50 \%$ pulse height cut remove a further $7 \%$ of the events. Timing fits are first applied to the events using the true muon direction. Figure 7.15 shows the 

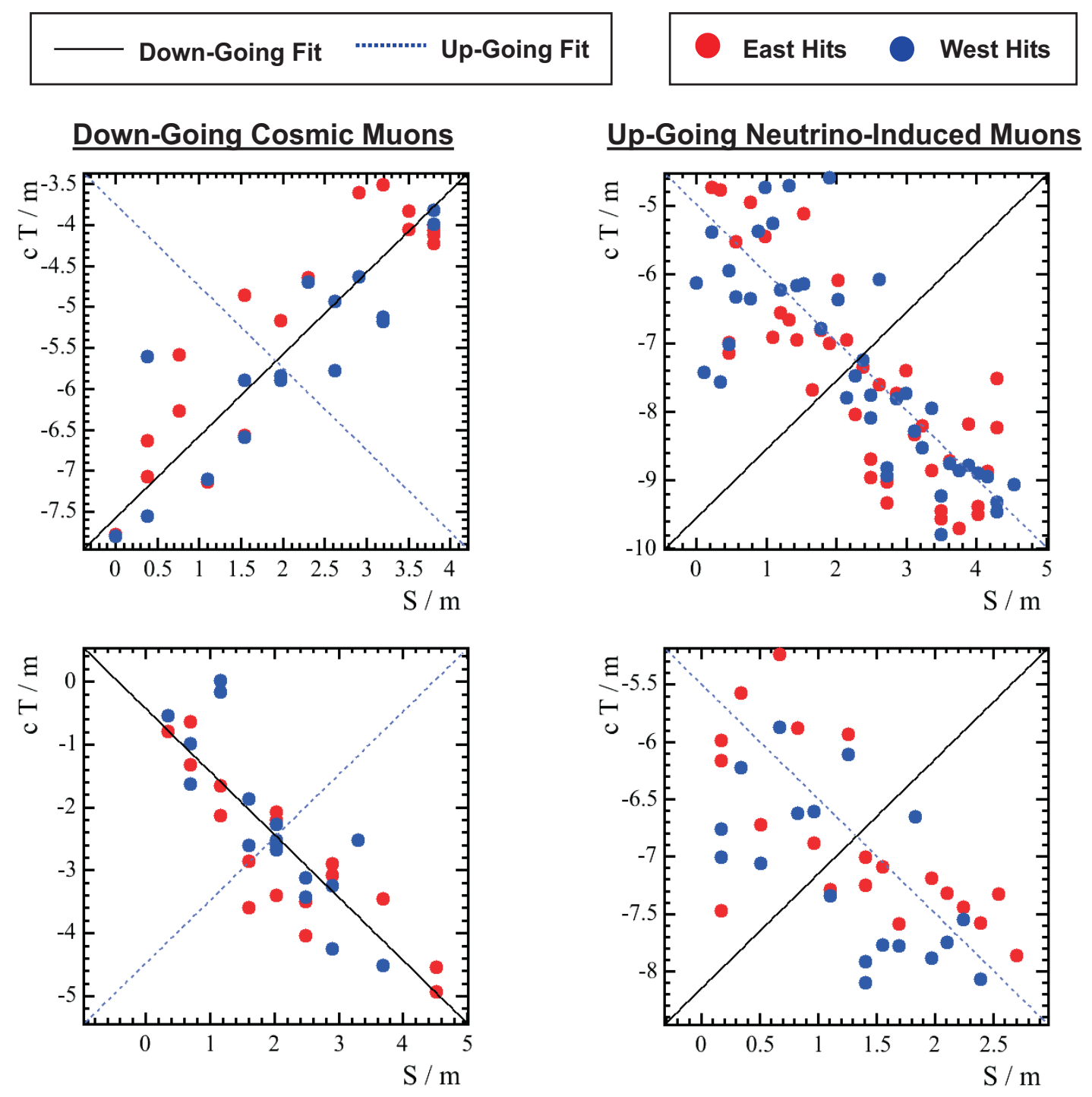

Figure 7.10: Examples of timing fits for reconstructed muon tracks in data. The measured times are plotted against the distances along the muon tracks. The black solid lines indicate the down-going timing fits, while the blue dotted lines indicate the up-going timing fits. The two time profiles on the left are for down-going stopping muon candidates, while the two time profiles on the right are for up-going neutrino-induced muon candidates. 


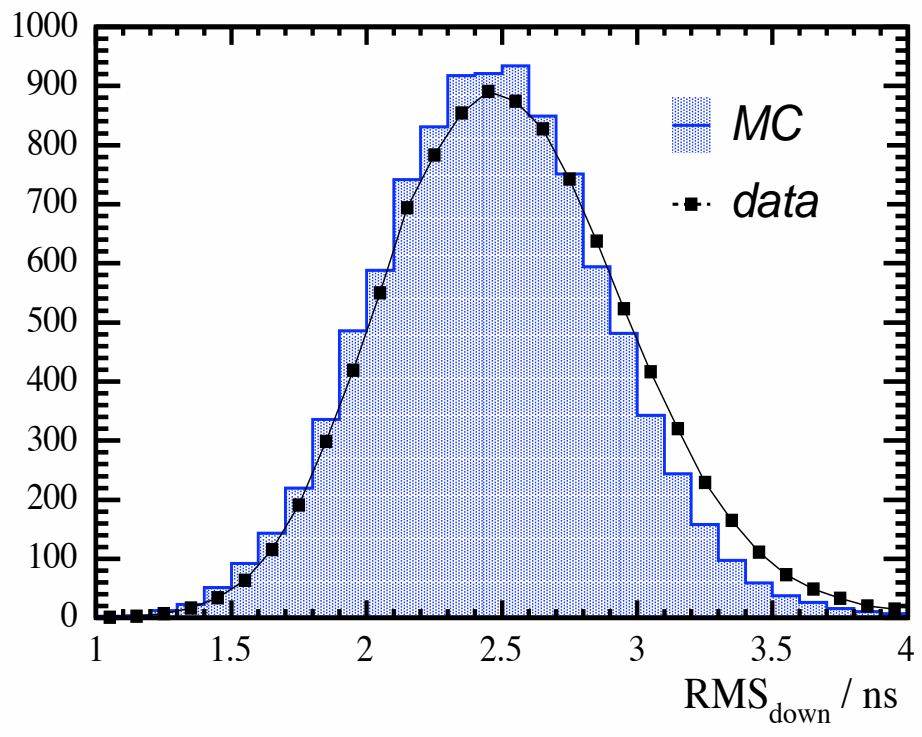

Figure 7.11: Distribution of rms deviations calculated for down-going timing fits to stopping muons.

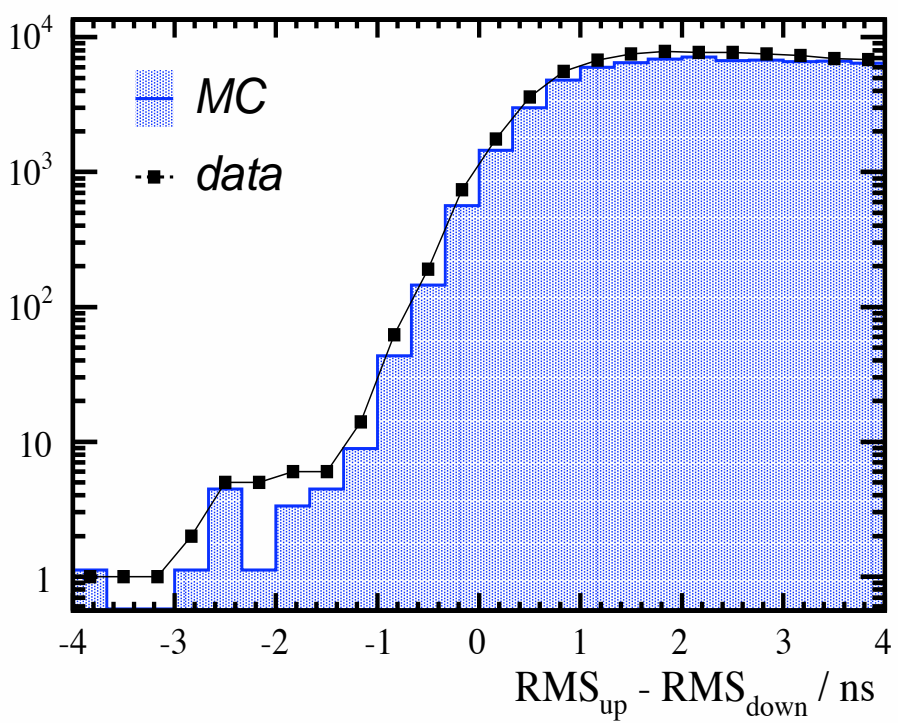

Figure 7.12: Distribution of differences between the rms deviations calculated for up-going and down-going timing fits to stopping muons. 


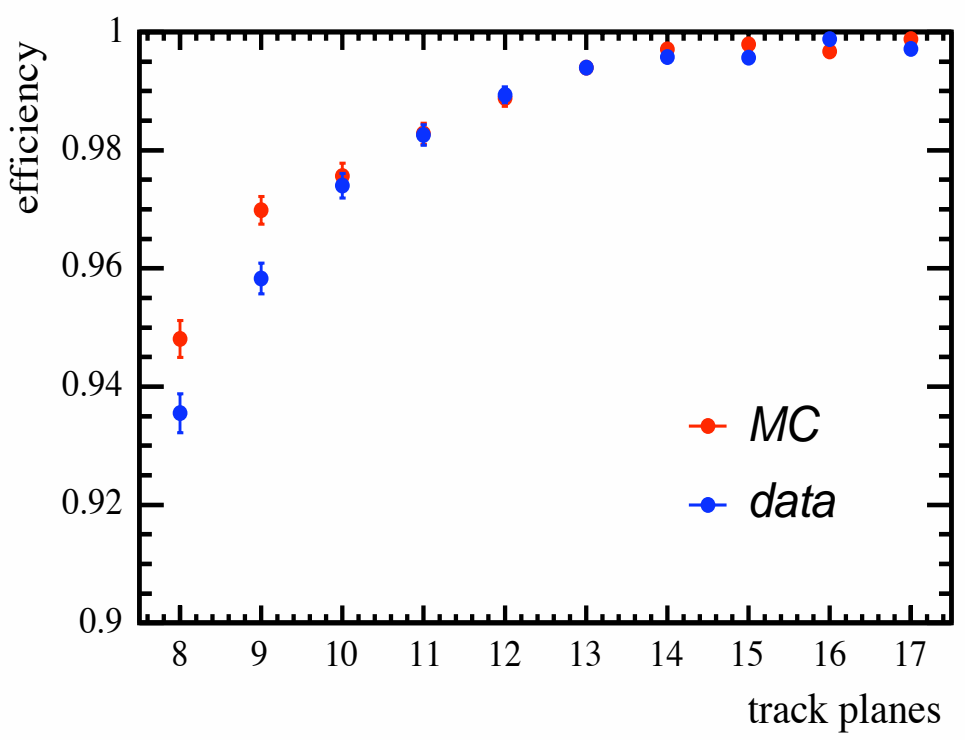

Figure 7.13: The efficiency for correctly reconstructing the direction of stopping muons as a function of the number of track planes.

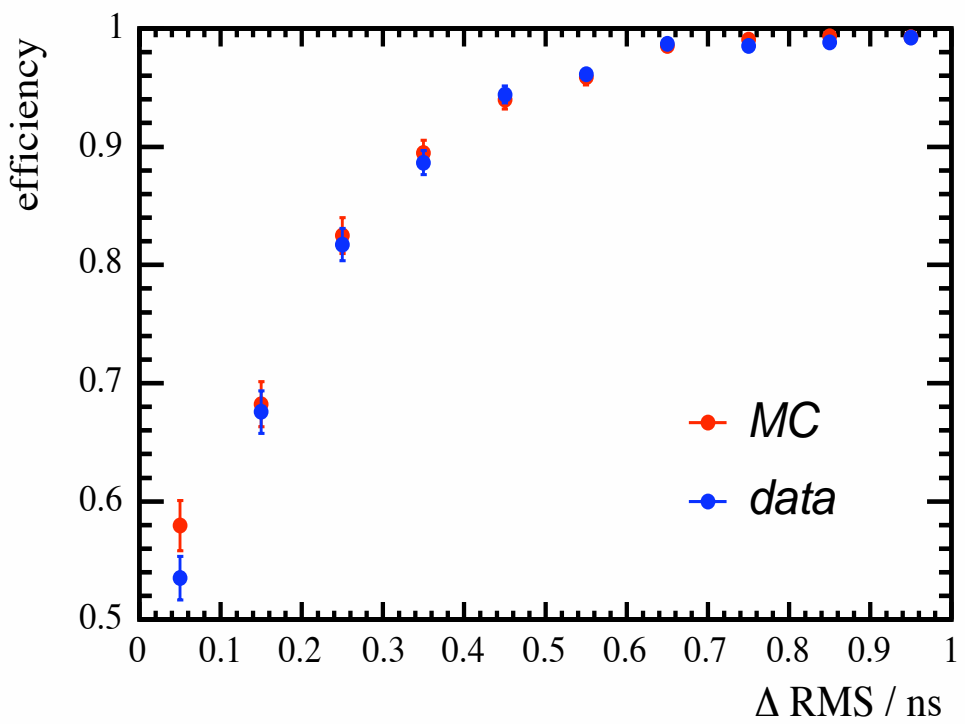

Figure 7.14: The efficiency for correctly reconstructing the direction of stopping muons as a function of the difference between the rms deviations for up-going and down-going timing fits to the muon tracks. 


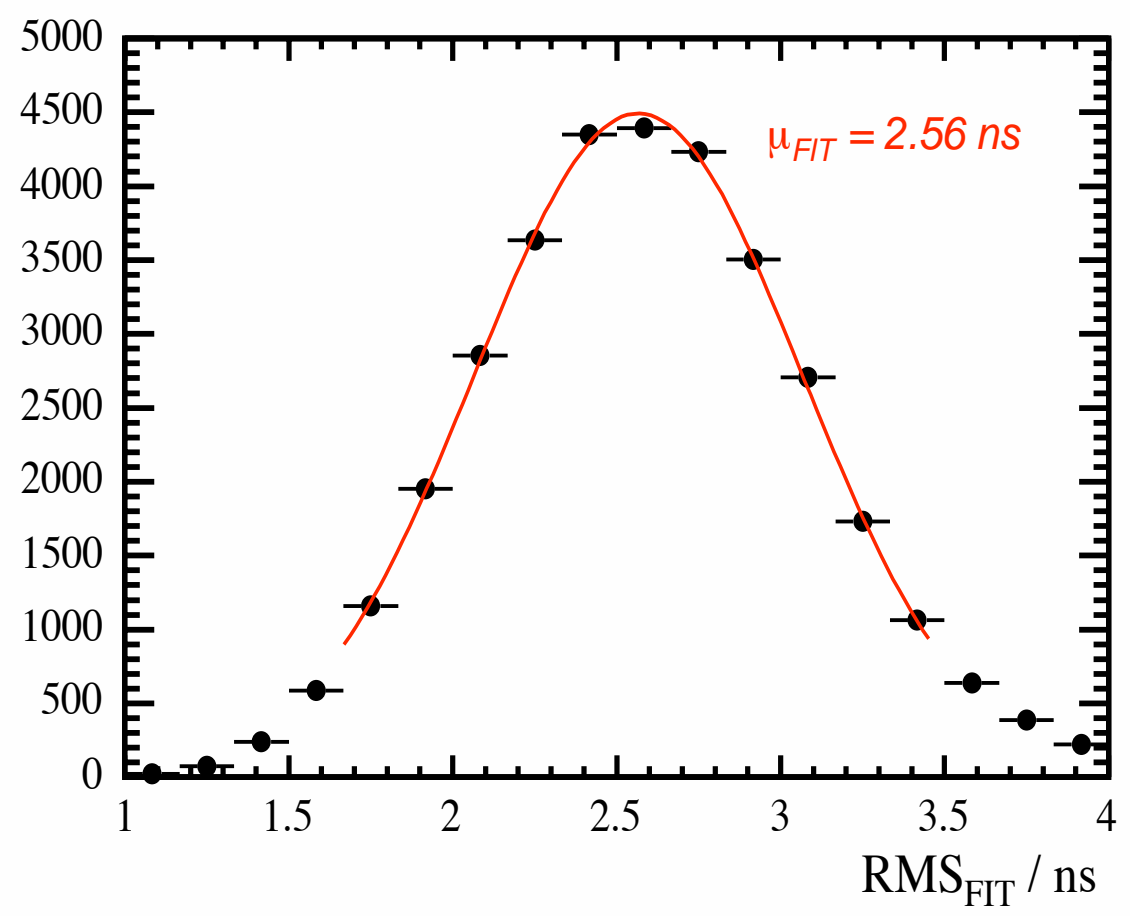

Figure 7.15: Distribution of rms deviations calculated for timing fits to $M C$ atmospheric $\nu_{\mu} / \bar{\nu}_{\mu} C C$ events using the true direction to perform the fits.

distribution of $r m s$ deviations for these timing fits. The mean $r m s$ deviation is calculated to be $2.56 \pm 0.04 \mathrm{~ns}$. This is close to the value calculated for stopping muons. Timing fits are then applied to the events fixing the muon direction as up-going and down-going. Figure 7.16 shows the distributions of $r m s_{u p}-r m s_{\text {down }}$ for events where the muon is known to be up-going and events where the muon is known to be downgoing. The two distributions are cleanly separated as a function of $r m s_{u p}-r m s_{\text {down }}$, with the majority of up-going muons lying in the range $r m s_{u p}-r m s_{\text {down }}<0$ and the majority of down-going muons lying in the range $r m s_{u p}-r m s_{\text {down }}>0$. The overall reconstruction efficiency is calculated to be $95.4 \%$. Figure 7.17 shows the reconstruction efficiency as a function of $\Delta r m s$. The efficiency rises from $50 \%$ at $\Delta r m s=0$, where the muon direction is ambiguous, to $>90 \%$ for $\Delta r m s>0.5$. The purity of the separation between up-going and down-going events can be improved by cutting on $\Delta r m s$. 


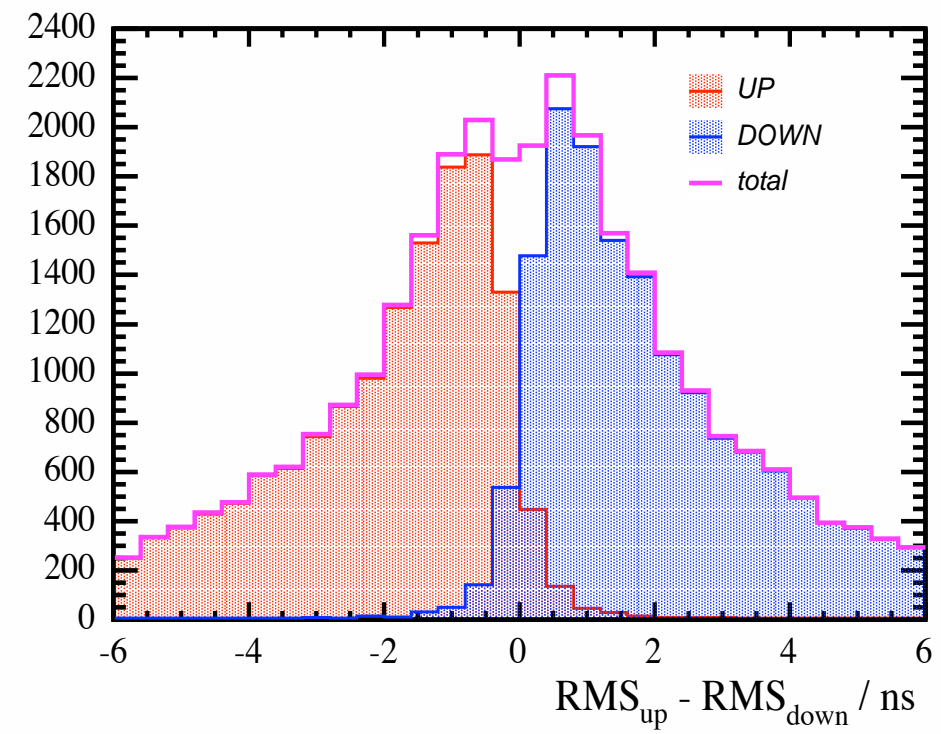

Figure 7.16: Distribution of differences between rms deviations for up-going and down-going timing fits to MC atmospheric $\nu_{\mu} / \bar{\nu}_{\mu} C C$ events.

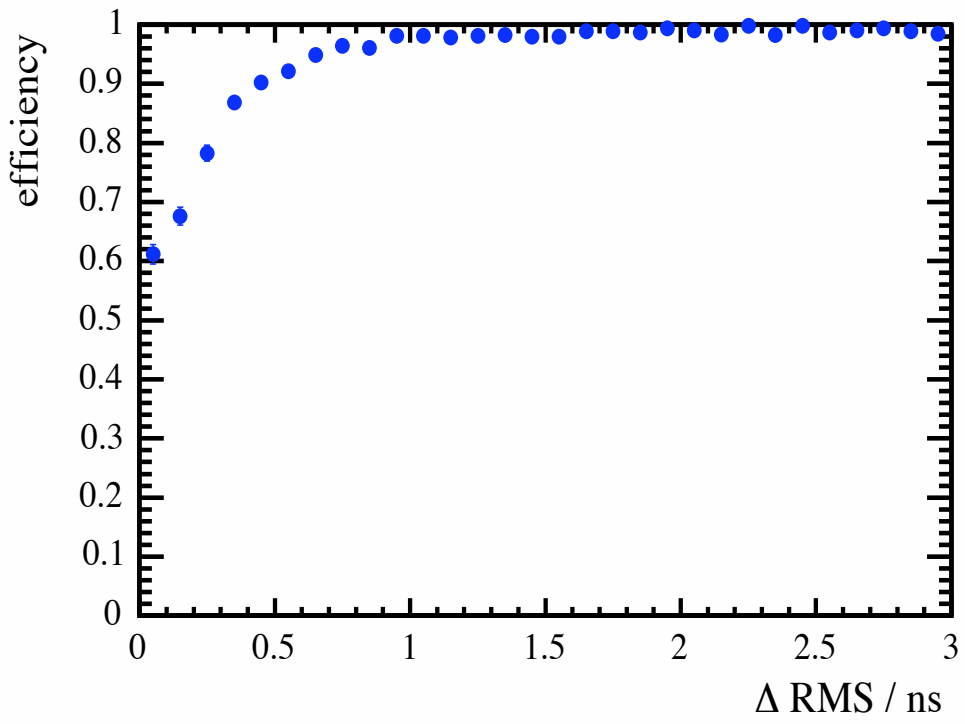

Figure 7.17: The efficiency for correctly reconstructing the direction of $M C$ atmospheric $\nu_{\mu} / \bar{\nu}_{\mu}$ CC events as a function of the difference between the rms deviations for the up-going and down-going timing fits to the reconstructed muon tracks. 


\subsubsection{Charge Reconstruction}

The Far Detector magnetic field enables the separation of charged current $\nu_{\mu}$ and $\bar{\nu}_{\mu}$ events based on the muon charge. The muon charge is determined by combining the direction and curvature of the reconstructed muon track.

The change in muon momentum along its trajectory is described as follows:

$$
\frac{d \mathbf{p}}{d s}(\mathbf{s})=0.3 Q \hat{\mathbf{p}}(\mathbf{s}) \times \mathbf{B}(\mathbf{s})+\hat{\mathbf{p}}(\mathbf{s}) \frac{d p}{d s}(p)
$$

where:

$\mathbf{p}(\mathbf{s})=$ muon momentum at position s along track $(\mathrm{GeV})$.

$\mathbf{B}(\mathbf{s})=$ magnetic ficld at position $\mathrm{s}$ along track $(\mathrm{T})$.

$Q=$ muon charge.

The first term represents the change in momentum due to muon propagation through the magnetic field; the second term represents the change in momentum due to muon cnergy loss. The equation is rearranged to give the muon charge as follows:

$$
\frac{Q}{p(\mathbf{s})}=\frac{\frac{d \hat{\mathbf{p}}}{d s}(\mathbf{s}) \cdot \hat{\mathbf{p}}(\mathbf{s}) \times \mathbf{B}(\mathbf{s})}{0.3|\hat{\mathbf{p}}(\mathbf{s}) \times \mathbf{B}(\mathbf{s})|^{2}}
$$

Each reconstructed track is divided into overlapping segments spanning 15 planes. Each segment is parametcrized separately in the $\mathrm{C}$ and $\mathrm{V}$ views 11 sing a quadratic fit. The fits are then combined to give 3D trajectories for each segment. Equation 7.4 is used to calculate a value of $Q / p$ and its error $\sigma_{Q / p}$ for each track segment. These values are then combined to give an mean $\langle Q / p\rangle$ and crror $\sigma_{\langle Q / p\rangle}$ for the muon track. The reconstructed muon charge $Q_{f i l}$ is given by the sign of $\langle Q / p\rangle$. A first order correction is applied to account for muon energy loss and obtain an estimate of the initial muon momentum $p_{0}$. Assuming a constant energy loss $k$ this is given as follows:

$$
\frac{Q}{p_{0}}=\frac{\left\langle\frac{Q}{p}\right\rangle}{1+\frac{1}{Q_{f i t}}\left\langle k s \frac{Q}{p}\right\rangle}
$$

The performance of the charge reconstruction is tested using the samples of stopping muons described in Section 7.4.1. The muon direction is fixed as down-going so that the measured charge reflects the measured curvature of the muon track. The ratio $\langle Q / p\rangle / \sigma_{\langle Q / p\rangle}$ is used to measure the quality of the charge determination and separate 
clean samples of positive and negative muons. Figure 7.18 shows the distribution of $\langle Q / p\rangle / \sigma_{\langle Q / p\rangle}$ for data and MC. Both distributions consist of broad peaks at $\pm \bar{\nu}$ corresponding to positive and negative muons. The positive peak is larger than the negative peak, reflecting the excess of positive particles generated by the mainly positive cosmic ray flux. The charge ratio is calculated to be $N_{+} / N_{-}=1.24 \pm 0.01$ for $\mathrm{MC}$ and $N_{+} / N_{-}=1.37 \pm 0.01$ for data. The charge ratio calculated in $\mathrm{MC}$ is in good agreement with the input value of $N_{+} / N_{-}=1.25$ used in MC simulation. The charge ratio calculated in data is higher than the MC (see Section 6.3.2). The MC distribution is divided into positive and negative muons. The distributions are cleanly separated as a function of $\langle Q / p\rangle / \sigma_{\langle Q / p\rangle}$, with the majority of positive muons lying in the region $\langle Q / p\rangle / \sigma_{\langle Q / p\rangle}>0$, and the majority of negative muons lying in the region $\langle Q / p\rangle / \sigma_{\langle Q / p\rangle}<0$. The overall efficiency for correctly reconstructing the muon charge is calculated to be $96.6 \%$. Figure 7.19 shows the reconstruction efficiency as a function of the muon energy. The efficiency initially rises with energy as the mean track length increases, reaches a peak of $>99 \%$ around $5 \mathrm{GeV}$, and then falls with energy as the mean track curvature decreases.

The performance of the charge reconstruction in atmospheric neutrinos is tested using the sample of MC atmospheric $\nu_{\mu} / \bar{\nu}_{\mu}$ CC events described in Section 7.4.1. The track direction is fixed using the true muon direction so that the measured charge reflects the measured curvature of the muon track. Figure 7.20 shows the distribution of $\langle Q / p\rangle / \sigma_{\langle Q / p\rangle}$ for these events. The distribution is divided into neutrinos and antineutrinos. The distributions are cleanly separated as a function of $\langle Q / p\rangle / \sigma_{\langle Q / p\rangle}$, with the majority of neutrinos lying in the region $\langle Q / p\rangle / \sigma_{\langle Q / p\rangle}<0$, and the majority of anti-ncutrinos lying in the region $\langle Q / p\rangle / \sigma_{\langle Q / p\rangle}>0$. The overall efficiency for correctly reconstructing the muon charge is calculated to be $91.8 \%$. Figure 7.21 shows the reconstruction efficiency as a function of $|\langle Q / p\rangle| / \sigma_{\langle Q / p\rangle}$. The efficiency rises from $50 \%$ at $|\langle Q / p\rangle| / \sigma_{\langle Q / p\rangle}=0$, where the muon charge is ambiguous, to $>90 \%$ for $|\langle Q / p\rangle| / \sigma_{\langle Q / p\rangle}>2.0$. The purity of the separation between neutrinos and antineutrinos can be improved by cutting on $|\langle Q / p\rangle| / \sigma_{\langle Q / p\rangle}$. A high purity of separation is required to account for the uneven rates of atmospheric neutrinos and anti-neutrinos. The event rate for neutrinos is approximately double the event rate for anti-neutrinos, enhancing the background of neutrinos in the selection of anti-neutrinos. 


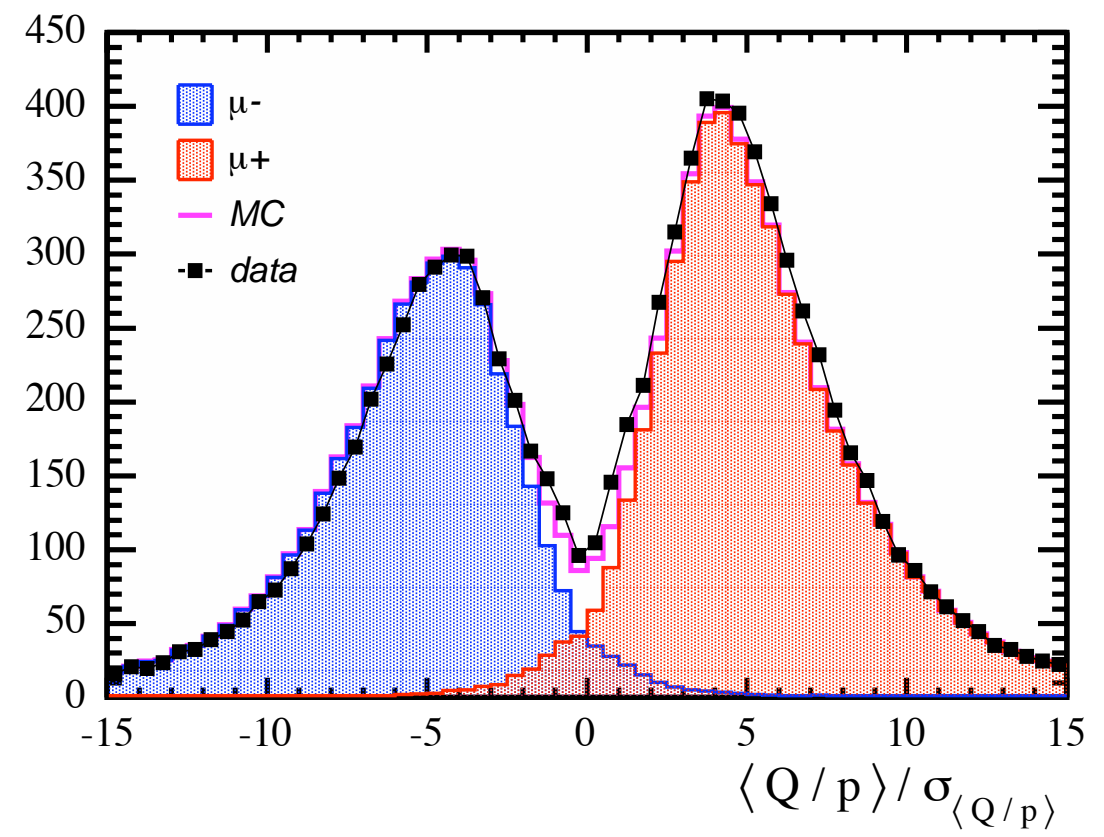

Figure 7.18: Distribution of $\langle Q / p\rangle / \sigma_{\langle Q / p\rangle}$ for stopping muons.

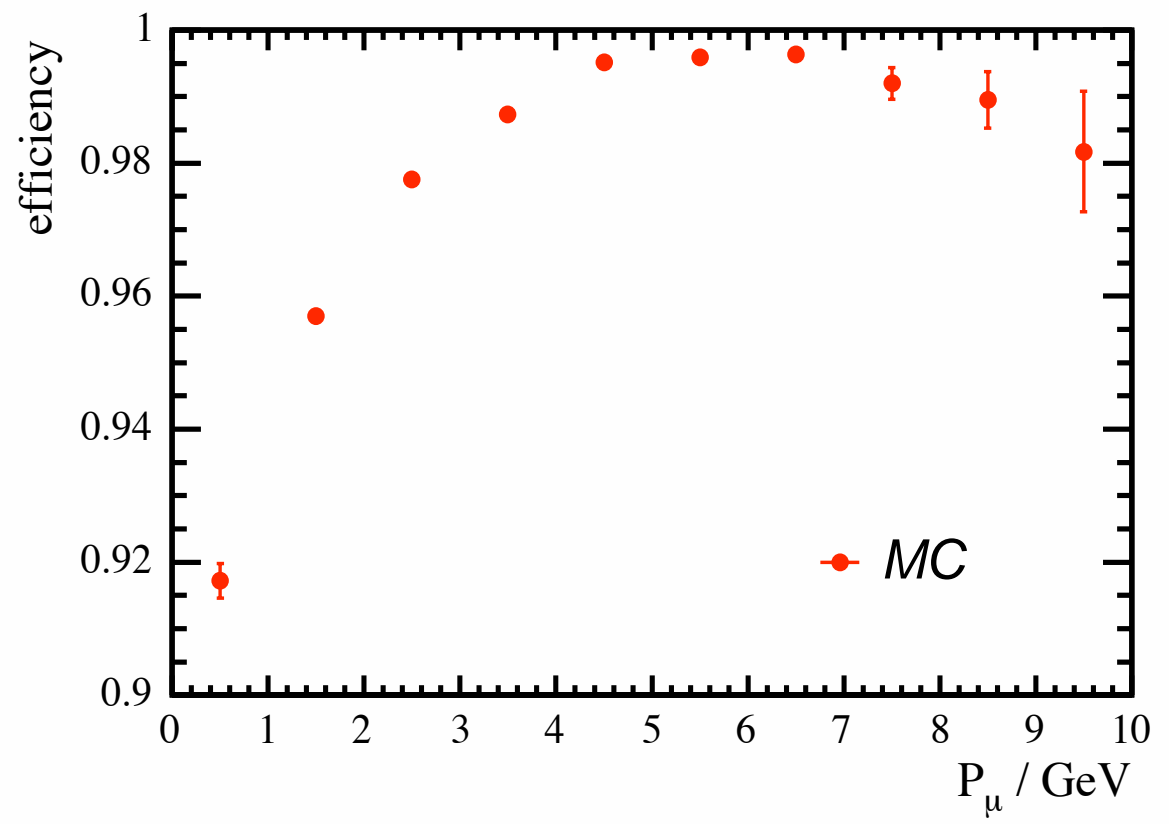

Figure 7.19: The efficiency for correctly reconstructing the charge of $M C$ stopping muons as a function of the true muon energy. 


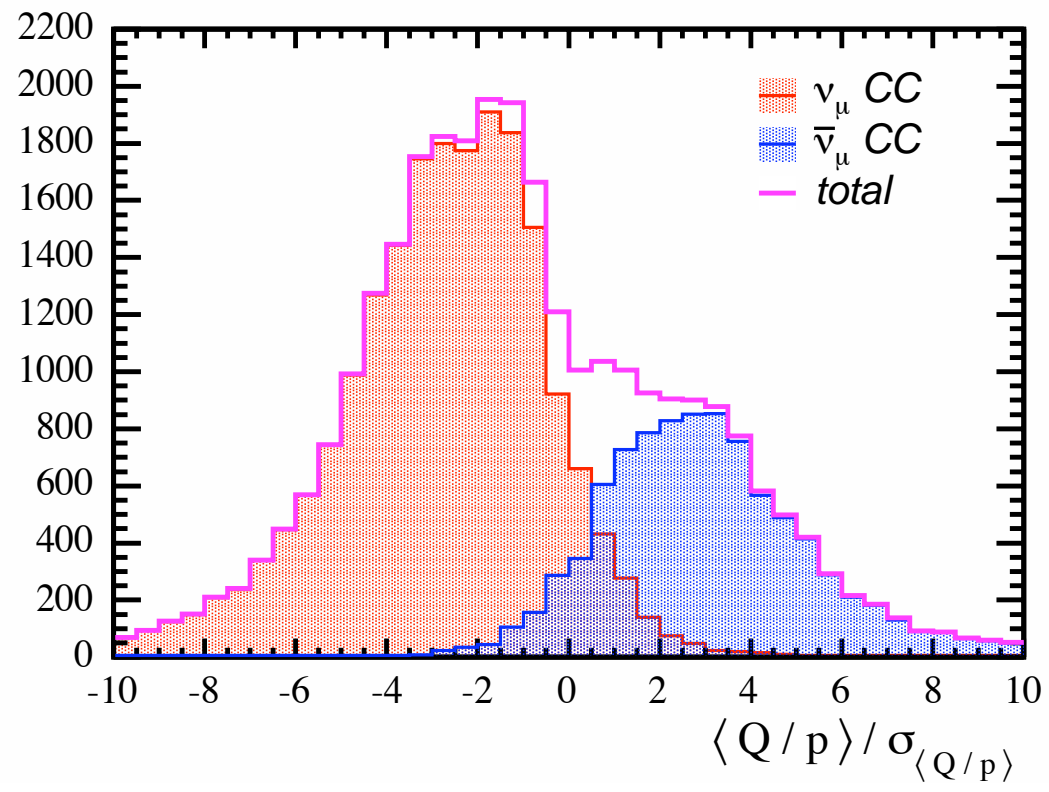

Figure 7.20: Distribution of the reconstructed values of $\langle Q / p\rangle / \sigma_{\langle Q / p\rangle}$ for the selected sample of $M C$ atmospheric $\nu_{\mu} / \bar{\nu}_{\mu} C C$ events.

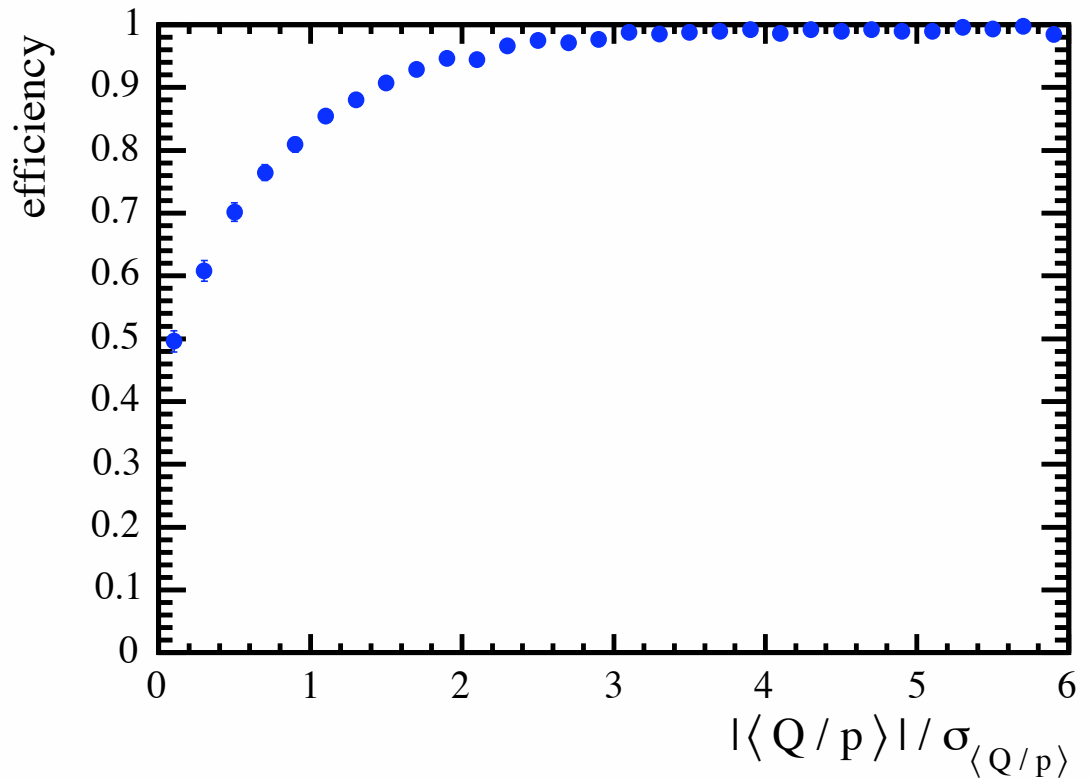

Figure 7.21: The efficiency for correctly reconstructing the muon charge in $M C$ atmospheric $\nu_{\mu} / \bar{\nu}_{\mu} C C$ events as a function of $|\langle Q / p\rangle| / \sigma_{\langle Q / p\rangle}$. 


\subsubsection{Energy Reconstruction}

The total energy of atmospheric $\nu_{\mu} / \bar{\nu}_{\mu}$ CC events is found by combining the muon momentum with the vertex shower energy. For muons which begin and end inside the detector, the muon momentum is determined from the range of the muon through the detector. For muons which exit the detector in flight, the muon momentum is determined from the curvature of the muon in the magnetic field. The vertex shower energy is determined by summing the total pulse height in the vertex shower.

For fully contained atmospheric $\nu_{\mu} / \bar{\nu}_{\mu}$ CC events, the muon momentum is determined from the range of the muon track through the detector. The range is found by linearly interpolating between the hits on the track and calculating the total distance travelled through the steel. The muon momentum is then parameterized as a linear function of the range through stecl. The momentum resolution is determined using fully contained muon tracks selected from the MC atmospheric $\nu_{\mu} / \bar{\nu}_{\mu}$ CC event sample. The tracks are required to begin and end $>0.5 \mathrm{~m}$ and $>5$ planes from the edges of the detector. Figure 7.22 shows the reconstructed momentum as a function of true momentum for the selected events. Figure 7.23 shows the percentage resolution and offset in the reconstructed momentum as a function of true momentum. The mean reconstructed momentum is in agreement with the true momentum at the level of $3 \%$ over the range $0-8 \mathrm{GeV}$. The small discrepancies are due to systematic effects in the reconstruction: at low energies, the reconstructed range is on average too long since tracks are cxtrapolated as far as possible into vertex showers; at high energies, the reconstructed range is on average too short due to muons that lose a significant proportion of their energy by bremsstrahlung. The momentum resolution is parametcrized as follows:

$$
\frac{\sigma_{P}}{P}=5.9 \% \otimes \frac{5.4 \%}{\sqrt{P}}
$$

The momentum resolution is worse at low energies due to the increased effect of sampling in the detector, fluctuations in muon energy loss, and multiple scattering.

For partially contained atmospheric $\nu_{\mu} / \bar{\nu}_{\mu}$ CC events, the muon momentum is determined from the curvature of the muon track in the magnetic field. Equation 7.5 is used to calculate a value of $Q / p_{0}$ for the reconstructed track. This is then inverted to give a measurement of the muon momentum. The momentum resolution is determined us- 
ing partially contained muon tracks selected from the MC atmospheric $\nu_{\mu} / \bar{\nu}_{\mu} \mathrm{CC}$ event sample. The tracks are required to begin $>0.5 \mathrm{~m}$ and $>\check{o}$ planes from the edges of the detector, and end $<0.5 \mathrm{~m}$ and $\leq 5$ planes from the edges of the detector. The calculated crror in the measured value of $\langle Q / p\rangle$ is required to be less than $50 \%$. Figure 7.24 shows the reconstructed momentum as a function of true momentum for the selected events. Figure 7.25 shows the percentage resolution and offset in the reconstructed momentum as a function of true momentum. The mean reconstructed momentum is found to under-estimate the true momentum at the level of $10 \%$ over the range $0-14 \mathrm{GeV}$. The momentum resolution is found to be:

$$
\frac{\sigma_{P}}{P}=25 \%
$$

This resolution is approximately constant over the momentum range.

The vertex shower energy is calculated from the total pulse height in the shower. The pulse height in cach strip is corrected for strip-to-strip variations and attenuation in the readout fibres. The shower energy is then parameterized as a linear function of the total pulse height. The energy resolution is determined using both fully and partially contained MC atmospheric $\nu_{\mu} / \bar{\nu}_{\mu}$ CC events. Figure 7.26 shows the reconstructed energy as a function of the true kinetic energy of the vertex shower for the selected events. Figure 7.27 shows the percentage resolution and offset as a function of the true encrgy. The mean reconstructed energy is found to be in agreement with the true energy at the level of $10 \%$ over the range $0-4 \mathrm{GeV}$. The energy resolution is parameterized as follows:

$$
\frac{\sigma_{L}}{E}=34 \% \otimes \frac{47 \%}{\sqrt{E}}
$$

The low resolution at these low energies is due to the coarse granularity of the detector and high degrec of fluctuations in the vertex showers. The showers are composed of low energy hadronic particles which propagate in all directions through the detector. The showers may also contain electromagnetic components arising from $\pi^{0}$ or $\gamma$ production. The multiplicity of possible final states, low kinetic energy of particles produced in the showers, and varying size of the electromagnetic components lead to large fluctuations in the energy deposited in the scintillator. 

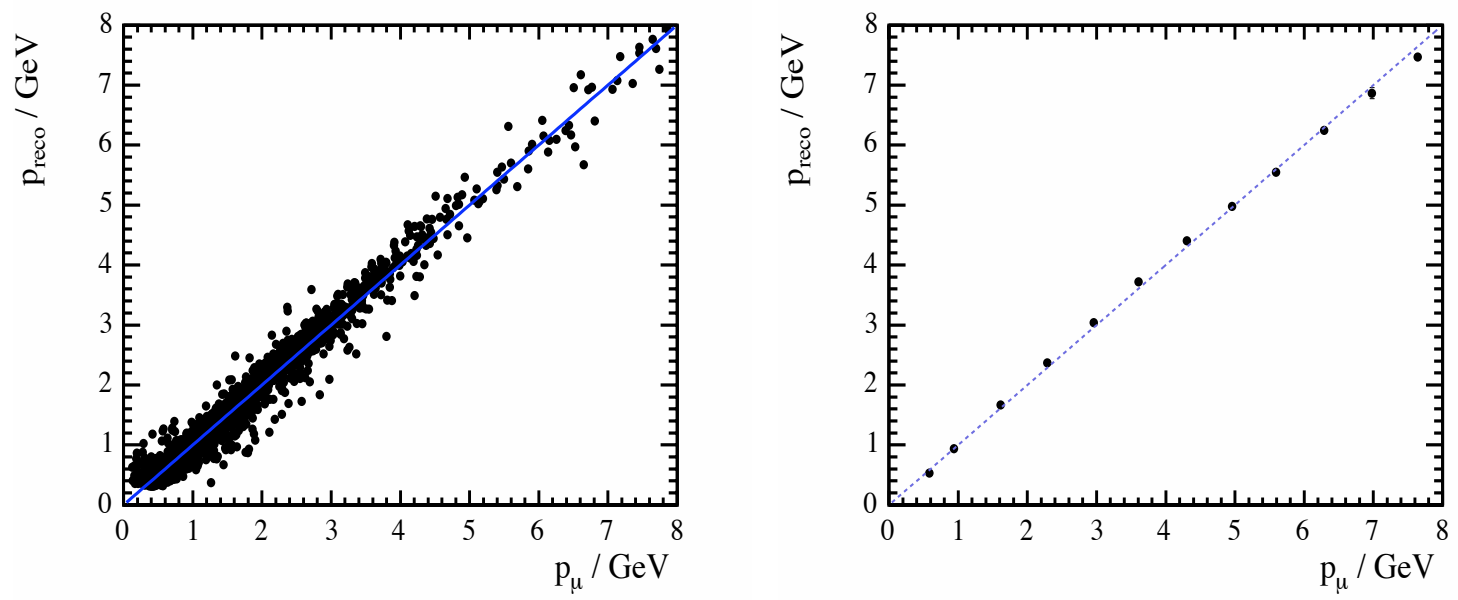

Figure 7.22: Muon momentum from range for fully contained $M C$ atmospheric $\nu_{\mu} / \bar{\nu}_{\mu} C C$ events. (a) reconstructed momentum vs true momentum (b) mean reconstructed momentum vs true momentum.
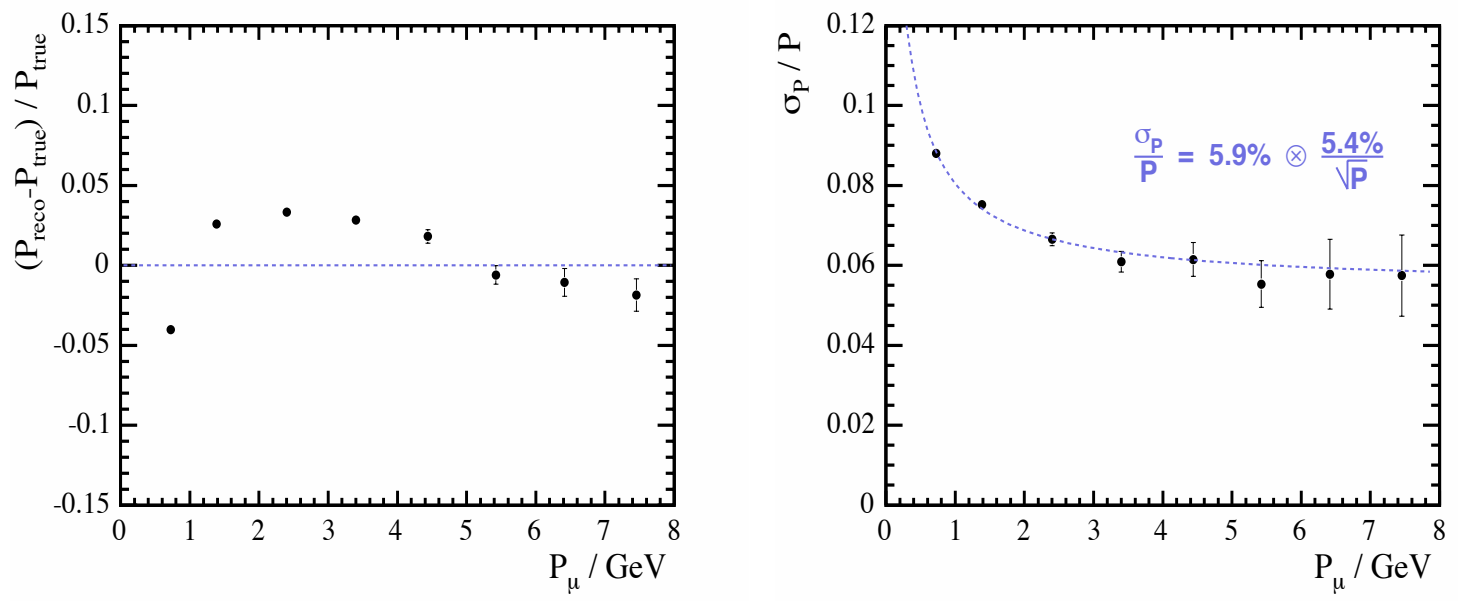

Figure 7.23: Muon momentum from range. (a) momentum offset vs true momentum. (b) momentum resolution vs true momentum. 

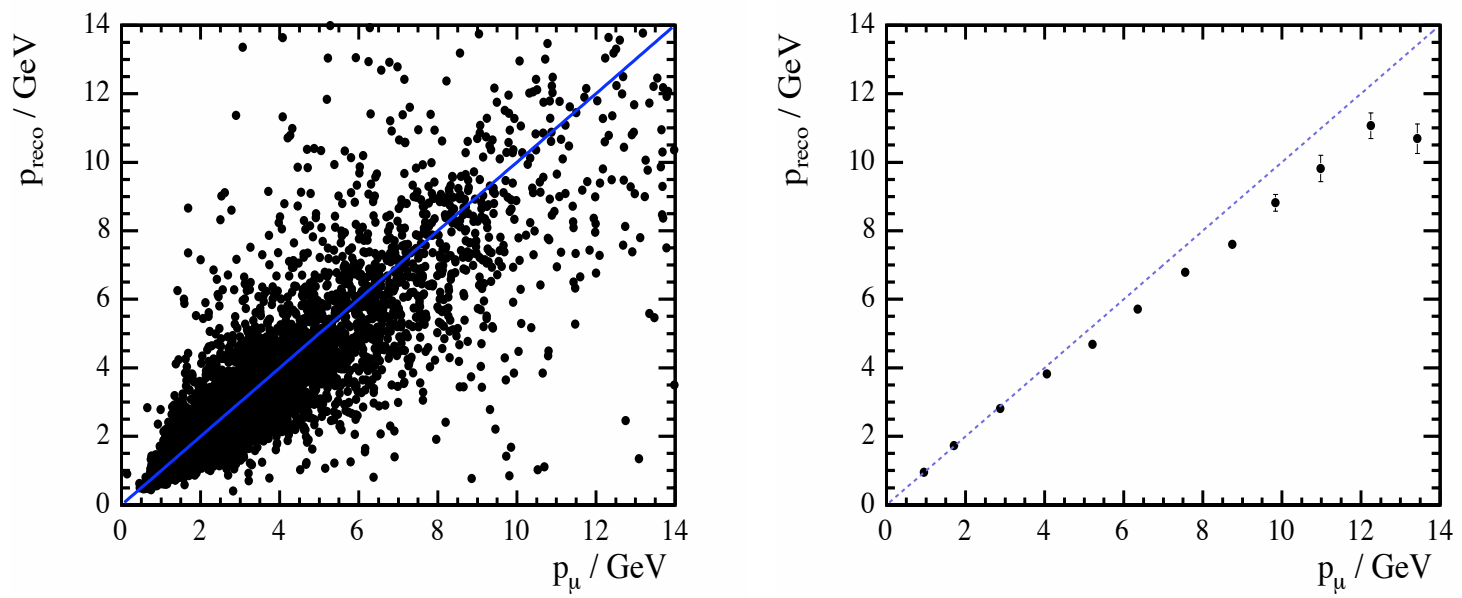

Figure 7.24: Muon momentum from curvature for partially contained $M C$ atmospheric $\nu_{\mu} / \bar{\nu}_{\mu} C C$ events. (a) reconstructed momentum vs true momentum. (b) mean reconstructed momentum vs true momentum.
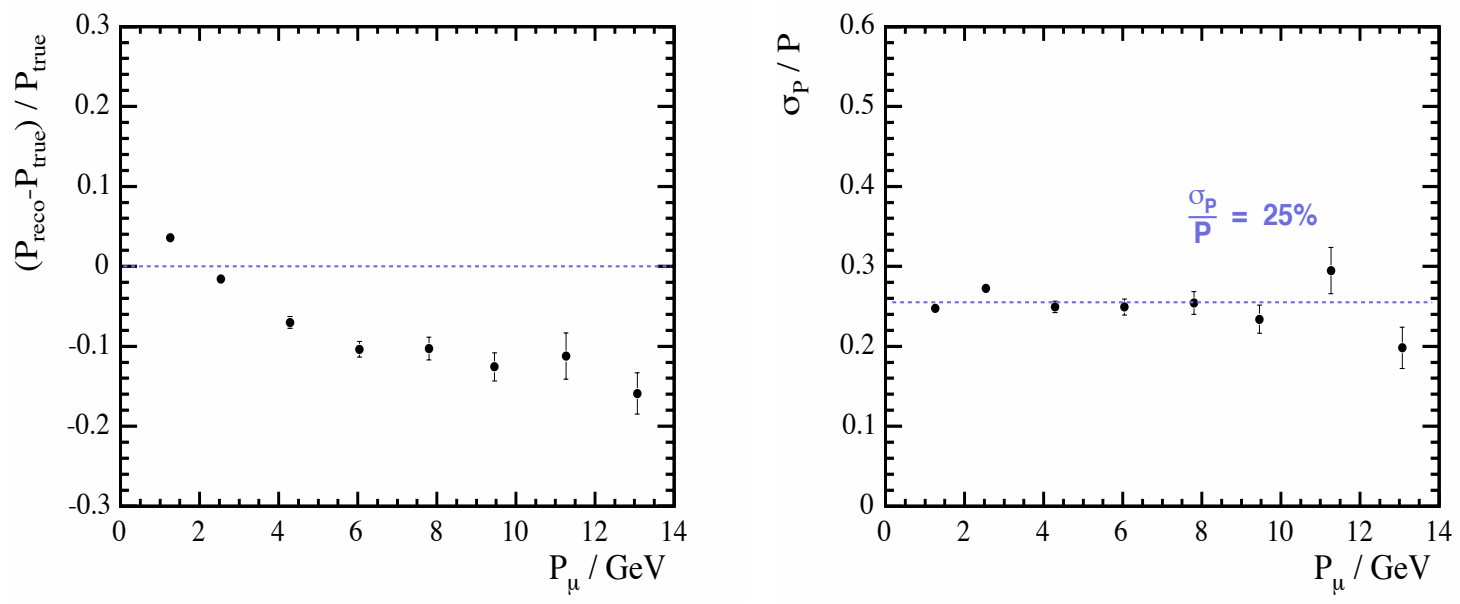

Figure 7.25: Muon momentum from curvature. (a) momentum offset vs true momentum. (b) momentum resolution vs true momentum. 

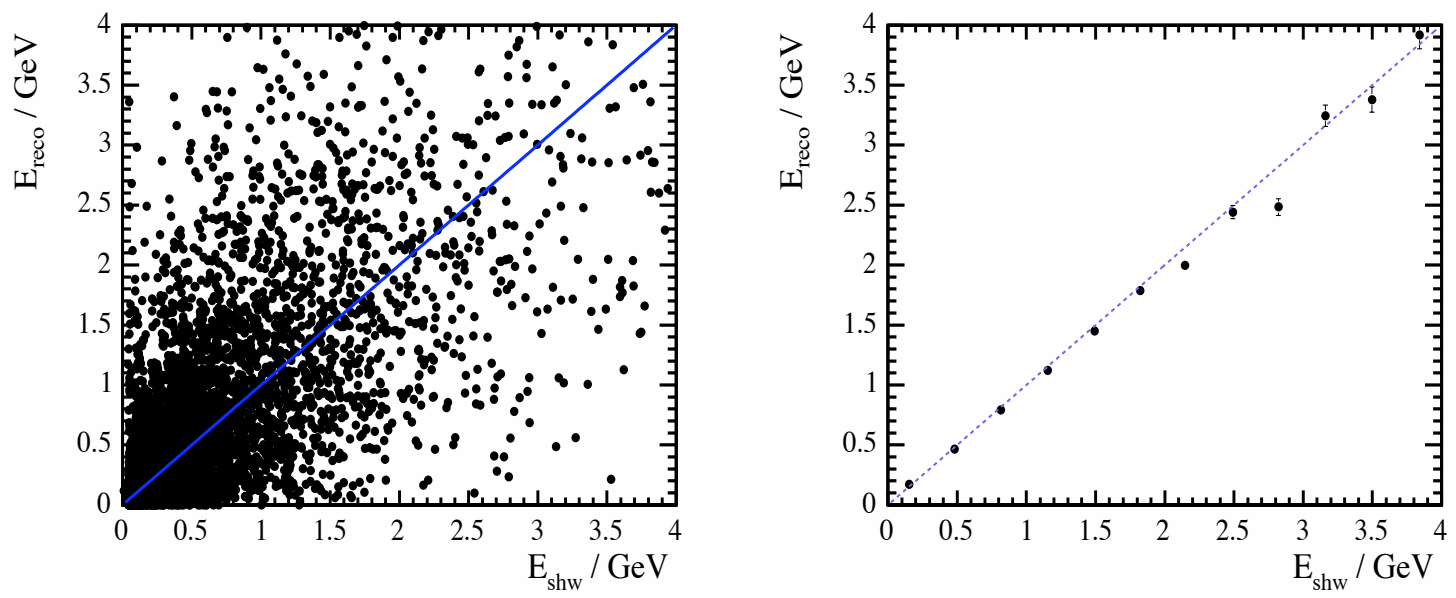

Figure 7.26: Reconstruction of shower energy from pulse height for $M C$ atmospheric $\nu_{\mu} / \bar{\nu}_{\mu}$ CC events. (a) reconstructed energy vs true shower energy. (b) mean reconstructed energy vs true shower energy.
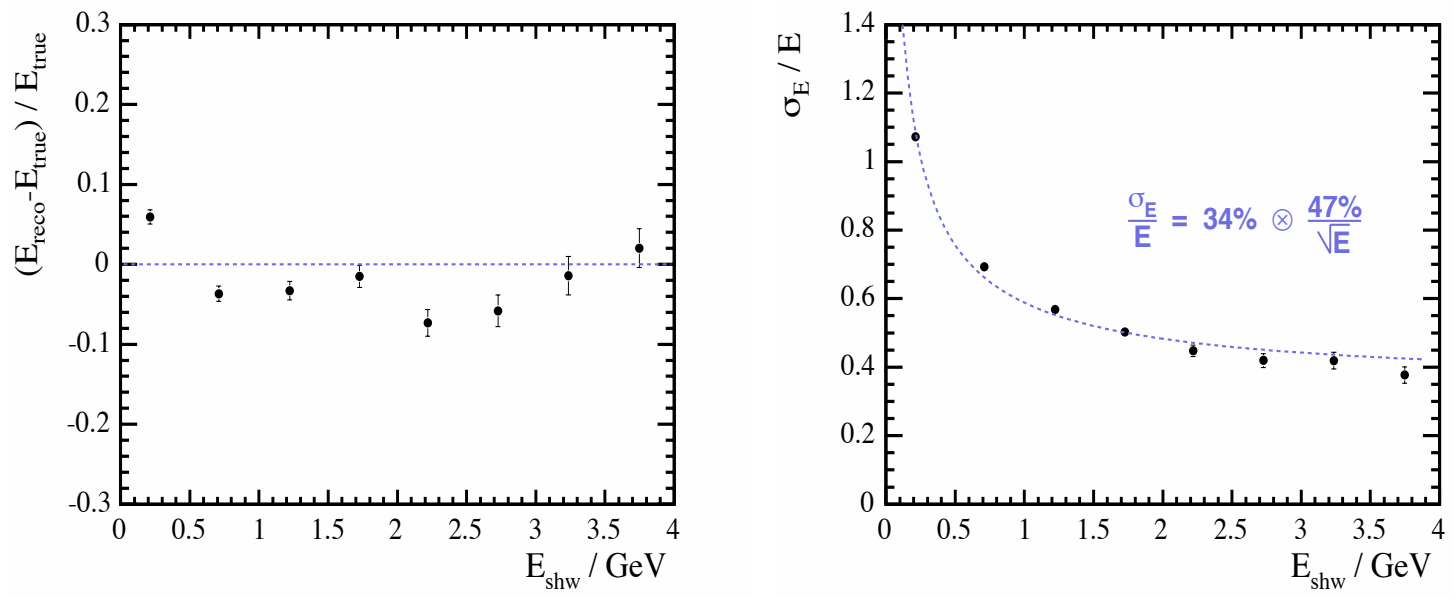

Figure 7.27: Determination of shower energy from pulse height. (a) energy offset vs true shower energy. (b) energy resolution vs true shower energy. 
The neutrino energy must be combined with the neutrino propagation distance to determine a value of $L / E$ for each event. The propagation distance is found by extrapolating the neutrino direction back to its production point in the atmosphere. For this analysis, the direction of the neutrino is approximated by the direction of the muon. Linear fits are applied in the $U$ and $V$ views to the first four planes of the muon track. The linear fits are then combined to give a measurement of the muon direction.

The angular resolution obtained using this method is measured using both fully and partially contained $\mathrm{MC}$ atmospheric $\nu_{\mu} / \bar{\nu}_{\mu} \mathrm{CC}$ events. Figure 7.28 shows the angular resolution for the selected sample of events. The blue histogram shows the distribution of angles between the reconstructed and true muon directions. The hatched histogram shows the distribution of scattering angles between the neutrino and muon directions. The angular resolution is calculated to be $7^{\circ}$, whereas the mean scattering angle is calculated to be $24^{\circ}$. The angular resolution is small compared with the intrinsic scattering between the neutrino and muon. Figure 7.29 shows the angular resolution and mean scattering angle as a function of neutrino cnergy. The mean scattcring angle falls stecply with energy as the boost between the centre of mass and laboratory frame increases. The angular resolution also falls with energy as the average muon curvature decreases improving the accuracy of the lincar approximation. The angular resolution is smaller than the mean scattering angle over the energy range $0-10 \mathrm{GeV}$.

This analysis employs simple and robust techniques of reconstructing the neutrino kincmatics. Improvements are possible by combining information from reconstructed muon tracks and vertex showers in a more sophisticated manner. Measurements of the track and shower kinematics can be combined with knowledge of the underlying physics of neutrino interactions to reconstruct the neutrino kinematics [146]. 


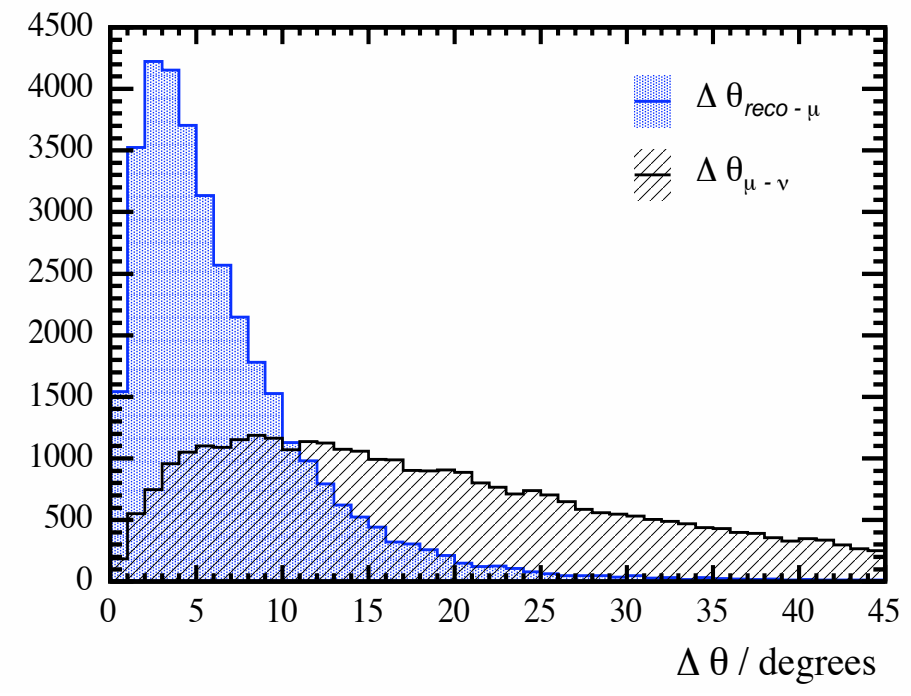

Figure 7.28: Angular resolution for $M C$ atmospheric $\nu_{\mu} / \bar{\nu}_{\mu} C C$ events. The neutrino direction is approximated by the direction of the reconstructed muon track. The blue histogram shows the distribution of angles between the reconstructed and true muon direction. The hatched histogram shows the distribution of angles between the neutrino and muon direction.

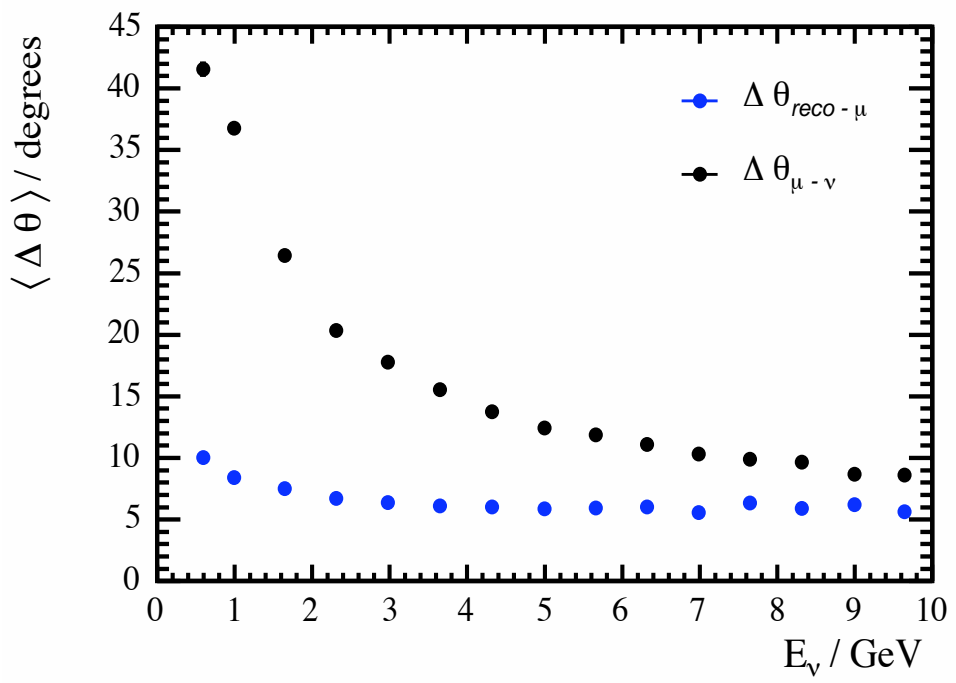

Figure 7.29: Angular resolution for $M C$ atmospheric $\nu_{\mu} / \bar{\nu}_{\mu} C C$ events vs neutrino energy. The blue markers represent the mean angle between the reconstructed and true muon direction. The black markers represent the mean angle between the neutrino and muon direction. 


\section{Chapter 8}

\section{Selection of Atmospheric Neutrino}

\section{Events}

\subsection{Event Selection Overview}

In order to perform a precise measurement of atmospheric neutrino oscillations, a clean sample of atmospheric $\nu_{\mu} / \bar{\nu}_{\mu}$ CC events must be selected from the data. The signature used to identify these events is a reconstructed muon track with an interaction vertex contained inside the fiducial volume of the detector. The fiducial volume is defined as the region $0.5 \mathrm{~m}$ from the edges of the detector, 5 planes from the ends of each supermodule and $0.4 \mathrm{~m}$ from the centre of the coil hole. This corresponds to $72 \%$ of the total detector volume giving a fiducial mass of $3.9 \mathrm{kT}$. The expected rate of atmospheric $\nu_{\mu} / \bar{\nu}_{\mu} \mathrm{CC}$ events which interact inside the fiducial volume of the detector and produce a muon which crosses $\geq 6$ planes is calculated to be 0.5 events/day. The main background to these events arises from cosmic muons. Even at a depth of 2100 mwe, cosmic muons are incident on the detector at a rate of 60,000 events/day. This is much larger than the rate of signal events. This background must be reduced by a factor of $>10^{6}$ in order to make an atmospheric neutrino oscillation analysis possible.

The selection of $\nu_{\mu} / \bar{\nu}_{\mu}$ CC events is performed in a number of stages. The majority of through-going cosmic muons are removed by a scries of filters that analyse events at the digit level. The events are then reconstructed, and events which begin inside the fiducial volume are selected. The cosmic muon background can be divided into two classes. For events that are down-going or have a contained upper vertex, the main 
background arises from cosmic muons that appear contained due to the geometry of the detector. Cosmic muons are peaked towards down-going vertical angles, parallel to the alignment of the planes. Cosmic muons incident on the detector between two planes can penetrate decp into the detector before entering the scintillator. This background is reduced by applying a series of containment cuts to ensure that events are correctly contained inside the fiducial volume. For events that are up-going and have a contained lower vertex, the main background arises from cosmic muons that are mis-reconstructed as up-going from timing information. This background is reduced by applying a series of timing cuts to ensure that events are correctly reconstructed as up-going.

Since the separation of signal and background depends on the containment of events, the following types of events are defined (as indicated in Figure 8.1):

- Fully Contained Events (FC) : Events with a muon track that begins and cnds inside the fiducial volume. The main background arises from stopping muons that appear contained.

- Down-Going Partially Contained Events (PCDN) : Events with a muon track that begins inside the fiducial volume, travels downwards through the detector, and exits the detector. The main background arises from through-going muons that appear contained.

- Up-Going Partially Contained Events (PCUP) : Events with a muon track that begins inside the fiducial volume, travels upwards through the detector, and exits the detector. The main background arises from stopping muons that are mis-reconstructed as up-going.

To scparate signal and background in FC and PCDN events, sclection cuts are applied to the topology of events to ensure that events are correctly reconstructed inside the fiducial volume. The remaining background is then tagged by analysing the hits in the veto shicld. To separate signal and background in PCUP events, selection cuts are applied to the timing measurements to ensure that the events are correctly reconstructed as up-going. The event selection is first developed on $\mathrm{MC}$ cosmic muons and $\mathrm{MC}$ atmospheric neutrinos before being applied to the data. 


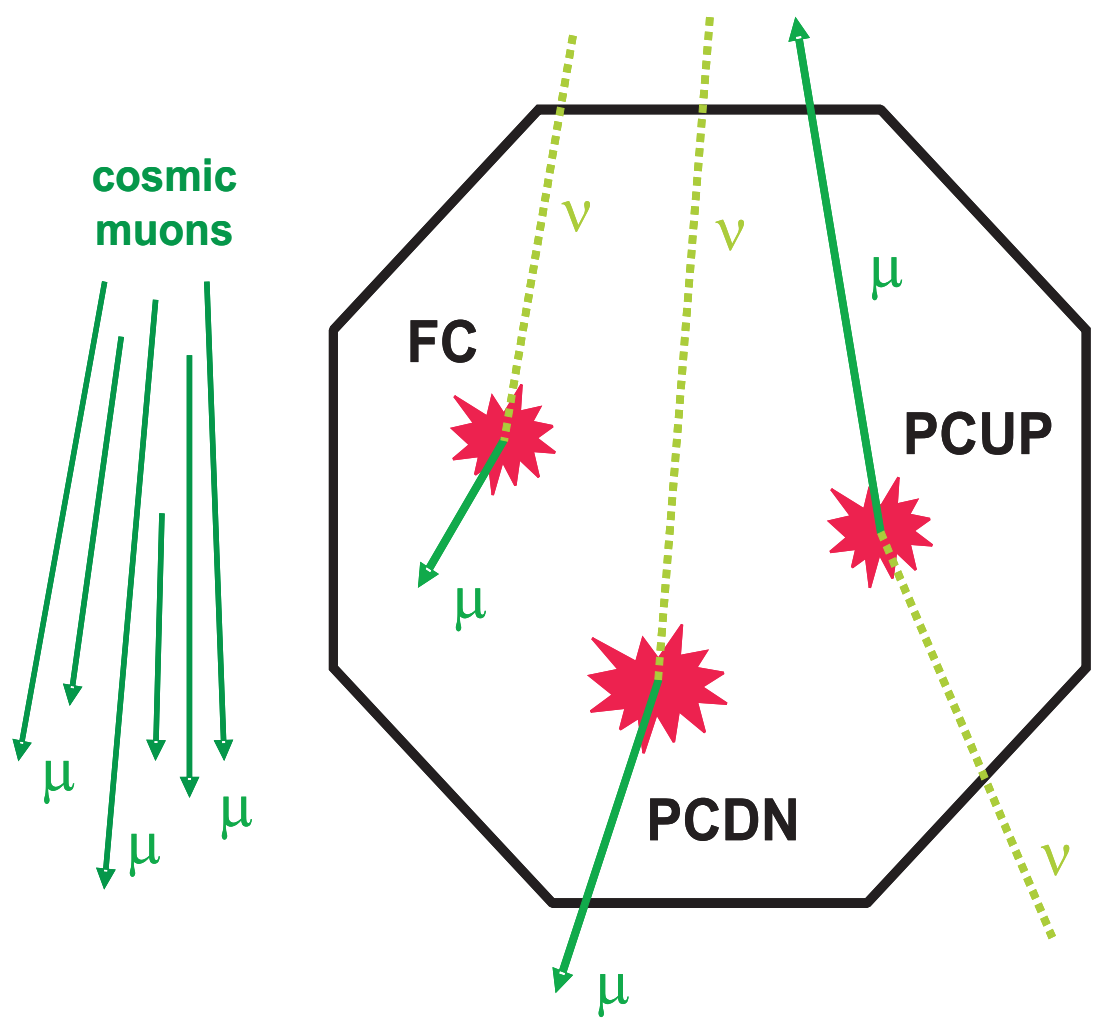

\begin{tabular}{|c|c|c|}
\hline Event Type & Main Background & Selection Cuts \\
\hline $\begin{array}{l}\text { Fully Contained } \\
\text { Events (FC) }\end{array}$ & \multirow{2}{*}{$\begin{array}{l}\text { Cosmic muons that } \\
\text { appear contained }\end{array}$} & \multirow{2}{*}{$\begin{array}{l}\text { Containment cuts } \\
+ \text { Veto shield }\end{array}$} \\
\hline $\begin{array}{l}\text { Down-Going Partially } \\
\text { Contained Events (PCDN) }\end{array}$ & & \\
\hline $\begin{array}{c}\text { Up-Going Partially } \\
\text { Contained Events (PCUP) }\end{array}$ & $\begin{array}{l}\text { Cosmic muons mis- } \\
\text { reconstructed as up-going }\end{array}$ & Timing cuts. \\
\hline
\end{tabular}

Figure 8.1: Atmospheric neutrino event classes. 


\subsection{Data and MC}

The Far Detector began collecting production data on 1st August 2003. This analysis is based on the data collected between 1st August 2003 and 31st October 2004. Only data collected under normal operating conditions is used in the analysis. The following requirements are used to select good data:

- Physics Data : The data are required to be part of physics runs, taken under normal trigger conditions, with the $2 / 36$ VARC trigger active in the VA electronics, and the $4 / 5$ plane trigger applied in the DAQ trigger farm.

- High Voltage On : The $8 \mathrm{HV}$ supplies that power the PMTs in the detector and veto shield are required to be powered up and operating at normal voltage.

- Coil Current On : The 2 coils that generate the magnetic field in each supermodule are required to be powered up and operating at normal current.

The total detector live time is found by counting the number of timeframes in the data set. This gives a total live time of 315.9 days, corresponding to an overall exposure of $4.67 \mathrm{kT}$-Yrs and fiducial exposure of $3.35 \mathrm{kT}$-Yrs. Figure 8.2 shows the daily and integrated live time as a function of the number of days since 1st August 2003. After the first 100 days, the daily live time rises to $>90 \%$ as the performance and understanding of the detector improves. The short intervals of detector down-time are due to maintenane and upgrades; specialized data-taking; and hardware malfunctions. The selected data set initially contains $140 \mathrm{M}$ detector triggers, consisting of $85 \%$ noise and natural radioactivity, and $15 \%$ cosmic muons. A clean sample of atmospheric neutrinos must be selected from this large background.

The event selection is developed using MC event samples (see Section 6.5). The central MC atmospheric neutrino sample uses the Barr et al model of the neutrino flux; the Neugen 3 model of neutrino interactions; and the GCALOR model of hadronic interactions. Additional samples are generated using different models to study systematic effects inherent in the choice of model. The central MC background sample contains 18.6 M cosmic muons (300 days live time). Additional samples are generated to measure small backgrounds from neutrons produced by muon spallation in the rock, and up-going muons produced by neutrino interactions in the rock. 

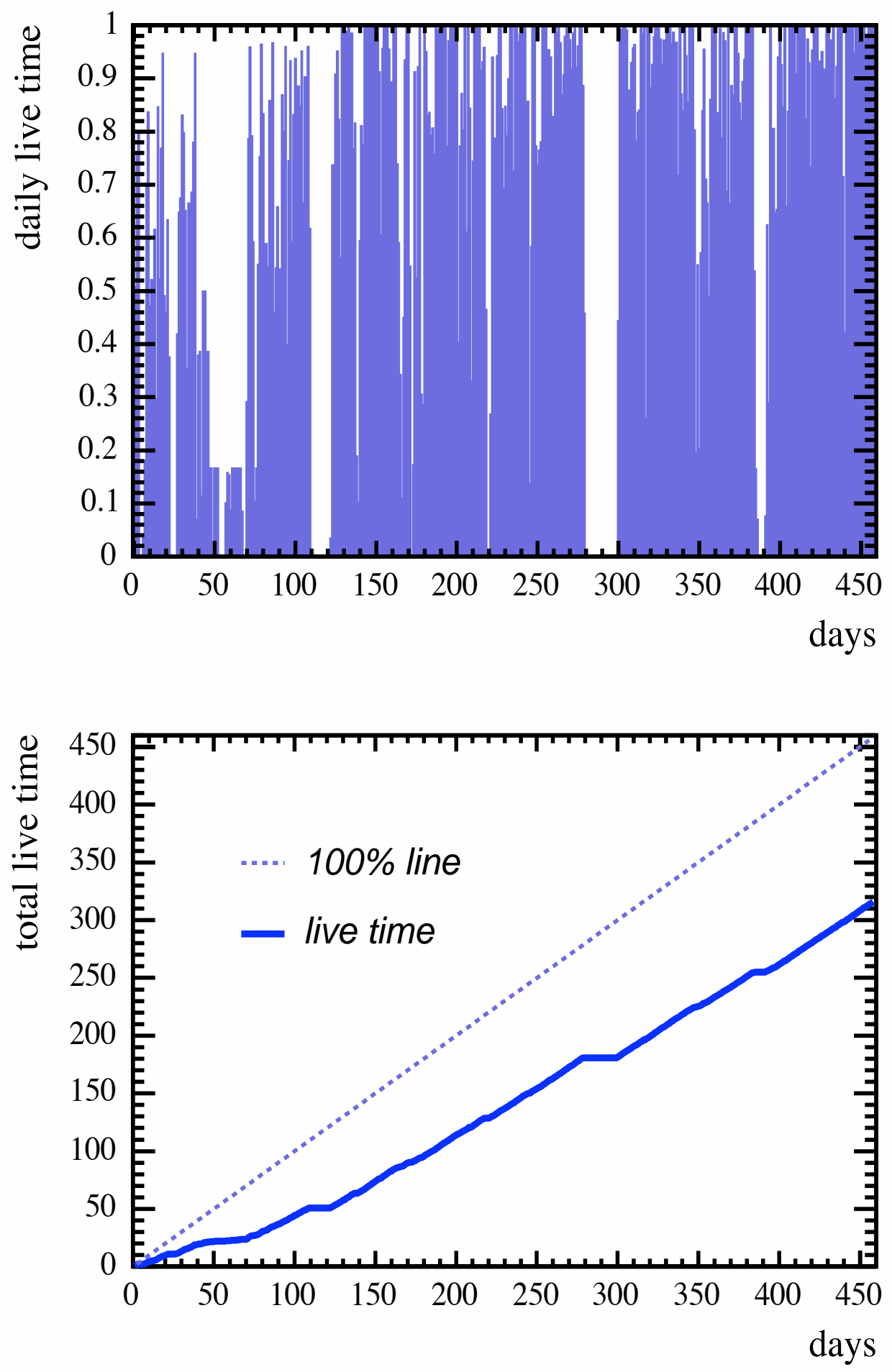

Figure 8.2: Daily and integrated live time for the selected data set as a function of days since 1st August 2003 when production data-taking began. 


\subsection{Low Level Filters}

\subsubsection{Hardware Filter}

At the level of $<1 \%$, events are split or truncated by the readout electronics and DAQ system. A cosmic muon that has been split or truncated can rescmble a contained event and therefore be selected as an atmospheric neutrino event. The following quality cuts are applied to the raw data to remove this background:

- Busy and Dead Channels : Contained events can be mimicked by cosmic muons passing through large regions of dead detector. It is important to identify the VA chips that are dead at the time of each event. VA chips can become dead for two possible reasons:

1. they are busy reading out signals on a P.MT.

2. they are dead due to a hardware malfunction.

Dead time is incurred each time a VA chip reads out a PMT. It takes $5 \mu$ s for the PMT photo-anodes to be read out by the VA chip and for the VA channels to be digitized by the VMM. This dead time is larger if multiple VA chips on a VMM are triggered. The VA chips that are busy at the time of an event are identified by analysing the hits in a $30 \mu$ s window preceding the event. The dead time of each VA chip triggered in this window is calculated, and a VA chip is considered busy if it precedes the event by less than this dead time. Readout holes in the detector are caused by malfunctioning hardware components. The VA chips that are dead at the time of an event are identified by analysing the trigger rates recorded for each VA chip at the end of each timeframe. A VA chip is considered dead if it has a trigger rate of less than $50 \mathrm{~Hz}$. The total number of busy and dead VA chips is used to identify events with large regions of dead detector. Events are rejected if the total number of busy and dead VA chips is greater than 20 .

- Light Injection : Each LI pulse creates a large number of busy VA chips in the detector. LI pulses are interspersed with normal data-taking at a rate of $50 \mathrm{~Hz}$. LI pulses are injected into groups of planes read out by a pair of opposite VME crates. This typically corresponds to a set of 64 continuous planes. The majority of pulses are injected into groups of strips read out by all 6 VA chips 
on a VMM. This produces the maximum possible dead time of $30 \mu \mathrm{s}$. Cosmic muon events passing through the planes during this period are truncated and can appear contained. Events that are closely preceded by LI pulses are removed by comparing the trigger times of events with the recorded times of TPMT hits. Events are rejected if they occur $<30 \mu$ s after a TPMT hit.

- Timeframe Boundaries : The raw data is broken into timcframes in order to be collected by the ROPs and processed by the TPs. The timeframes are created by splitting the data stream at the end of each second. If an event occurs close to the end of a timeframe, it can spill over into the subscquent timeframe. Although the hits in each event are timestamped within a window of $<100$ ns, it can take up to $50 \mu \mathrm{s}$ for the hits in an event to be funnelled through the VA electronics to the ROPs. If a new timeframe is created during this time, the hits will be split between the old and new timeframes. When an event is split in two, one half of the event can appear contained. In order to remove these events from the data, events are rejected if they occur $<100 \mu$ s from the end of a timeframe.

\subsubsection{Noise Filter}

The vast majority of triggers occur due to detector-induced noise or natural radioactivity. Noise-induced activity also occurs within events. Filters are applied at the digit level to remove the noise-induced events and the noise-induced digits within events.

Noise-induced events are filtered by cutting on the size of events. Figure 8.3 shows the distribution of the total number of digits in events for MC cosmic muons and data. The data contains both cosmic muons and noise-induced events. For events containing $<20$ digits, the data exceeds the MC by up to $10^{5}$ due to noise. Events are rejected if they contain $<10$ digits. This reduces the data set by $87 \%$ from $140 \mathrm{M}$ to $17 \mathrm{M}$ events.

Noise-induced activity within events is filtered by removing digits displaced from the event in space or time. The main body of digits satisfying the $4 / 5$ trigger condition is first identified. This contains all the digits in regions where 4 out of 5 contiguous planes are occupied. Digits are then removed from the event if they are displaced from this main body of digits by $>10$ planes, or if they occur at a time $>50$ ns before or $>500 \mathrm{~ns}$ after its earliest digit. 


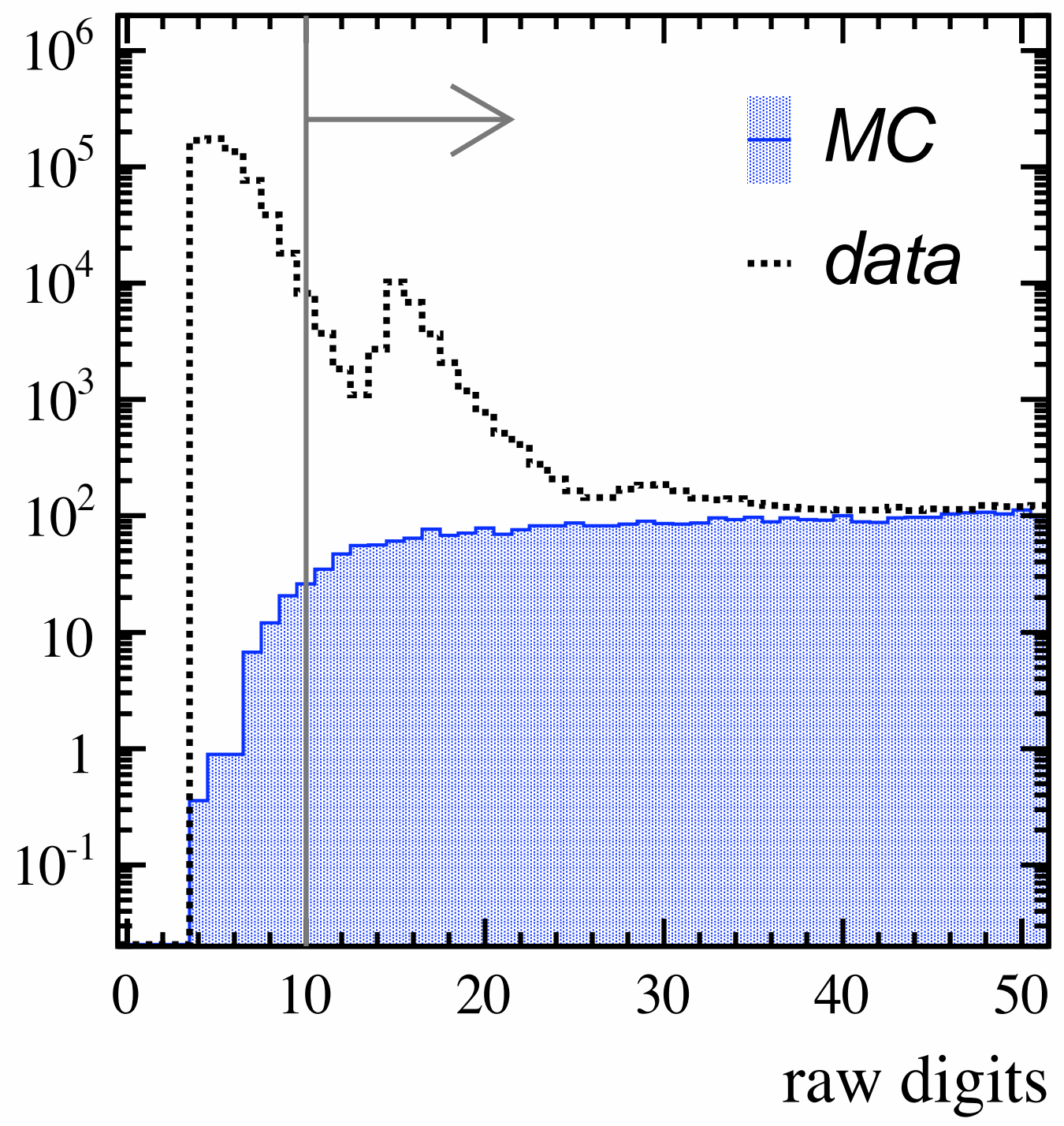

Figure 8.3: Distribution of raw digits for data and $M C$ cosmic muons. For events containing a small number of digits, the data exceeds the $M C$ by up to five orders of magnitude due to detector activity generated by noise and natural radioactivity. 


\subsubsection{Containment Filter}

A set of cuts on the length and containment of events is applied at the digit level to remove the majority of through-going cosmic muons from the data. For each event, a set of 3D digits is formed by combining each strip in the event with the strips of the opposite view in the adjacent planes. Events are rejected if they span a small number of planes or contain significant activity beside two or more edges of the detector. An enlarged fiducial volume is defined as the region $0.3 \mathrm{~m}$ away from the edges of the detector and $\bar{\jmath}$ planes away from the ends of each supermodule. The exterior volume of the detector is divided into edge regions corresponding to the 8 edges and 2 ends of each supermodule. The total pulse height in the fiducial volume and in each edge region is determined. Events are required to contain $>10$ PEs inside the fiducial volume, and $\geq \check{\jmath}$ planes with $>2$ PEs. The number of edge regions containing $>6$ PEs is then used to classify the containment of events as follows (sec Figure 8.4):

- Events with 0 edges are tagged as Fully Contained.

- Events with 1 edge are tagged as Partially Contained.

- Events with $\geq 2$ non-adjacent edges are tagged as Through-Going.

(an extra check is carried out on events with 2 adjacent edges to recover PC events that have exited between 2 cdges. If the distance between the mean positions in cach edge is $<1.33 \mathrm{~m}$, the adjacent edges are treated as 1 edge and the event is tagged as $\mathrm{PC}$ ).

Figure 8.5 shows the total pulse height in each edge region for data and MC. The sharp peak at $<10 \mathrm{PEs}$ is due to noise-induced activity whereas the broad peak at $50 \mathrm{PEs}$ to due to cosmic muons. The excess in data at $<50$ PEs is due to activity from the rock not modelled by the MC simulation. Figure 8.6 shows the total pulse height inside the fiducial volume for data and $\mathrm{MC}$. The distributions are in good agreement, with broad peaks at $1200 \mathrm{PEs}$ due to through-going cosmic muons.

Only events tagged as FC or PC are selected. This removes the majority of remaining noise-induced events and through-going muons, reducing the data set from $17 \mathrm{M}$ to $3.8 \mathrm{M}$ events. The efficiency for selecting atmospheric $\nu_{\mu} / \bar{\nu}_{\mu}$ CC events which interact inside the $0.5 \mathrm{~m}$ fiducial volume and deposit energy in $\geq 5$ planes is $99.5 \%$. 

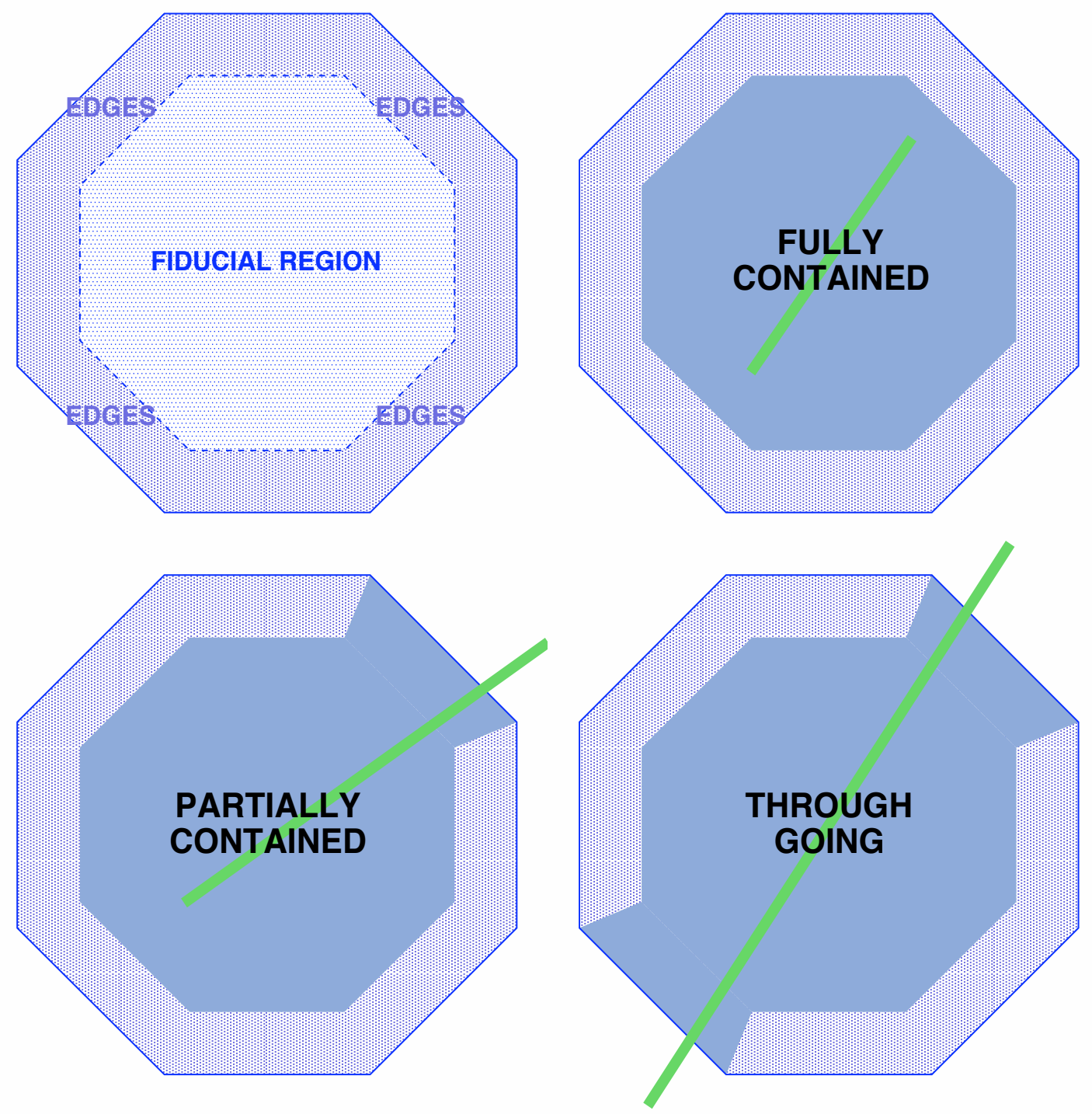

Figure 8.4: Classification of event containment using 3D hits. (a) Events with activity beside 0 edges are tagged as full contained. (b) Events with activity beside 1 edge are tagged as partially contained. (c) Events with activity beside $\geq 2$ non-adjacent edges are tagged as through-going. 


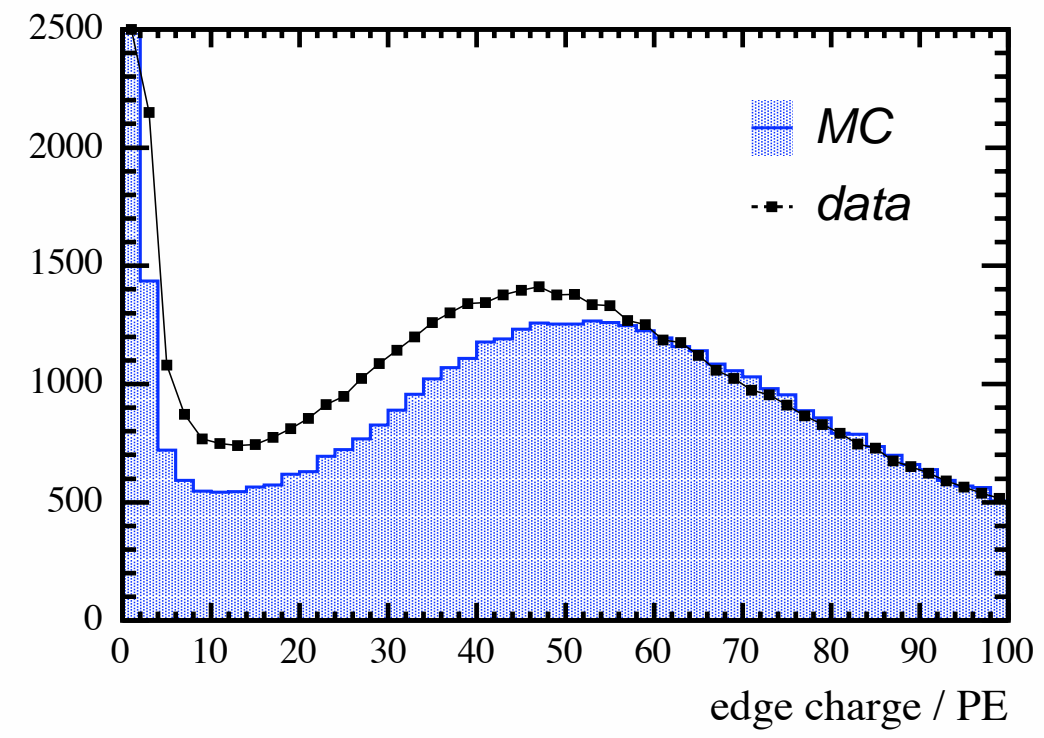

Figure 8.5: Distribution of pulse height contained in each edge region of detector. The data are represented by the black points, $M C$ cosmic muons are represented by the blue histogram.

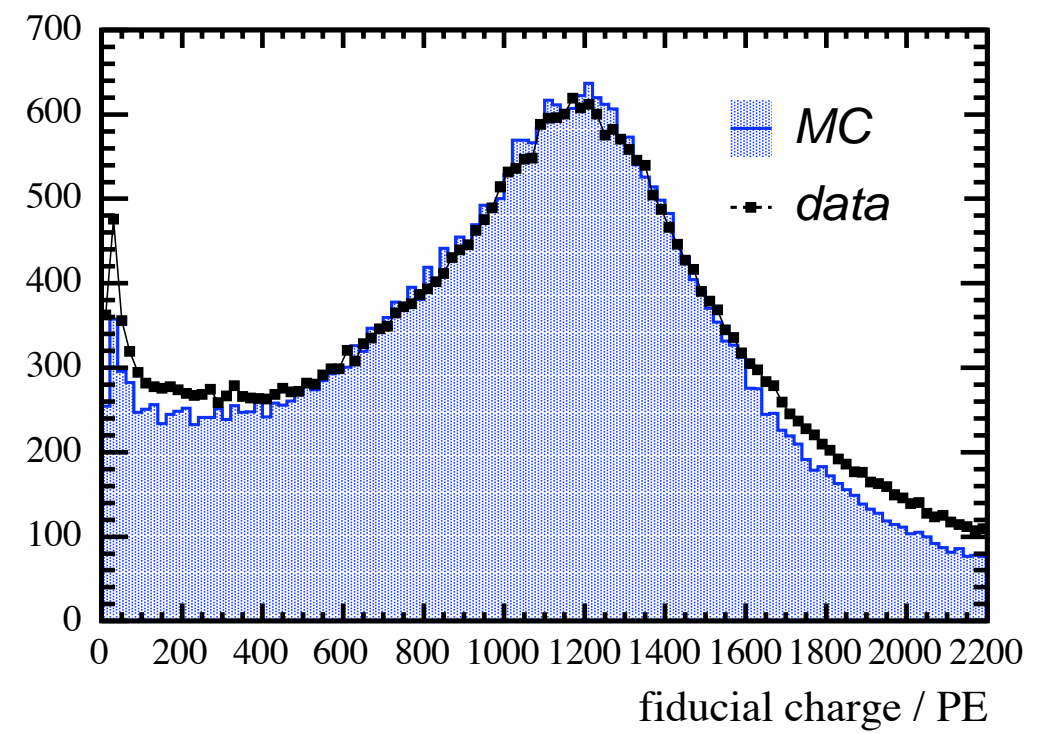

Figure 8.6: Distribution of pulse height contained in the enlarged fiducial region of detector. The data are represented by the black points, MC cosmic muons are represented by the blue histogram. 


\subsection{Selection of Tracks}

The signature of an atmospheric $\nu_{\mu} / \bar{\nu}_{\mu} \mathrm{CC}$ event is a muon track with a contained vertex. Events containing single tracks are selected from the data. The track vertex is required to be inside the fiducial volume of the detector. Figure 8.7 shows the distance between the track vertex and nearest detector edge for data, $\mathrm{MC}$ cosmic muons, and MC atmospheric $\nu_{\mu} / \bar{\nu}_{\mu}$ CC events which interact inside the fiducial volume. The fiducial cuts remove $>95 \%$ of the cosmic muons. To ensure that events are well reconstructed, the following cuts are also applied to the tracks:

- Track Length : Tracks are required to cross $\geq 3$ planes in each view and $\geq 8$ planes in total. This length cut introduces an energy threshold on muons corresponding to $300 \mathrm{McV}$ for muons travelling perpendicular to the planes.

- Track Quality : A series of quality cuts are applied to the tracks. Tracks are required to contain $\geq 5$ track-like planes, defined as planes which contain a total pulse height of $<80 \mathrm{PEs}$, with $>80 \%$ of the pulse height located within \pm 1 strip of the track. This ensures that the track contains a clean set of track-like strips and is well reconstructed. The total pulse height in the region \pm 1 strip of the track is also required to be $>50 \%$ of the pulse height in the event. This removes contained tracks produced by high energy showers. Finally, all planes in the event are required to contain $<500$ PEs. This removes cosmic muons which enter the detector at a steep angle, deposit a large amount of encrgy in a single plane, and scatter out of the plane to produce a contained track.

The fiducial and quality cuts reduce the data set to 36,000 events, compared with an expected atmospheric $\nu_{\mu} / \bar{\nu}_{\mu}$ CC signal of 140 events. In order to further reduce the cosmic muon background, the data are divided into FC/PCDN/PCUP events based on the containment of the muon track. For FC events, the muon is required to begin and end inside the fiducial volume; for PCDN events, the muon is required to begin inside the fiducial volume, travel downwards, and end outside the fiducial volume; for PCUP events, the muon is required to begin inside the fiducial volume, travel upwards, and end outside the fiducial volume. The signal-to-background ratio for each event type is: $1: 150$ for FC events, $1: 500$ for PCDN events, and $1: 40$ for PCUP events. 


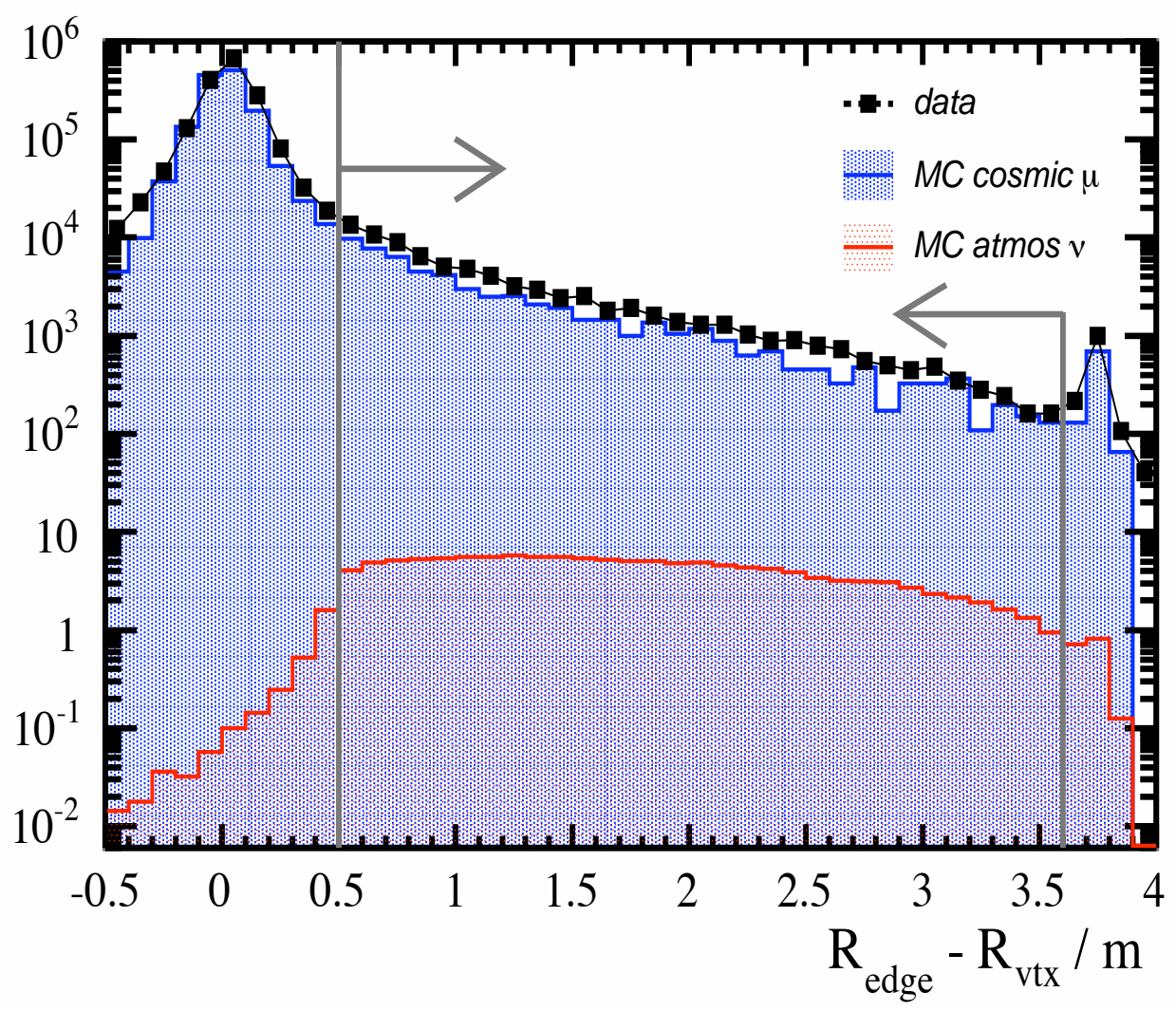

Figure 8.7: Distance between the track vertex and nearest detector edge for data, $M C$ cosmic muons and $M C$ atmospheric $\nu_{\mu} / \bar{\nu}_{\mu} C C$ events which interact inside the fiducial volume. Events are required to pass the track length and track quality cuts. The track vertex is required to occur $>0.5$ $m$ from the detector edge and $>0.4 m$ from the centre of the coil hole. 


\subsection{Selection of FC and Down-Going PC Events}

\subsubsection{Containment Cuts}

The FC and PCDN signal is separated from the background by applying a series of containment cuts to the events. The main background arises from cosmic muons that enter the detector at small angles to the planes and appear contained in the detector. The background is reduced by analysing the topology of events and applying cuts to ensure that events are correctly contained in the detector.

The same set of containment cuts is used to select both FC and PCDN events. Since the cosmic muon background is down-going, the containment cuts are applied to the upper vertex of the muon tracks. An additional track length cut is also used to suppress the high PCDN background. This is because the PCDN background arises from through-going muons, whereas the FC background arises from stopping muons. Therefore PCDN tracks are required to be $\geq 10$ planes and $>1 \mathrm{~m}$ in length.

The following containment cuts are applied to select FC and PCDN events:

- Trace : The dominant background arises from cosmic muons that are incident on the detector at steep angles and enter the scintillator a long way from the edge of the detector. The majority of cosmic muons follow straight trajectories. To remove these events, the muon track is projected linearly back to the detector edge to find the muon entry point. The horizontal distance $\Delta_{Z}$ (termed the trace) between the track vertex and muon entry point is calculated (sec Figure 8.8). Figure 8.9 shows the $\Delta_{Z}$ distribution for data, MC cosmic muons and MC atmospheric $\nu_{\mu} / \bar{\nu}_{\mu}$ CC events. The cosmic muon background is peaked at $\Delta_{Z}=0.1$, whereas the atmospheric neutrino signal is approximatcly uniform. Events are required to satisfy $\Delta_{Z}>0.5 \mathrm{~m}$. This removes approximately $95 \%$ of the cosmic muons and approximately $10 \%$ of the atmospheric neutrinos, reducing the signal-to-background ratio to $1: 10$ for both $\mathrm{FC}$ and $\mathrm{PCDN}$ events.

- Topology : Most of the remaining background arises from cosmic muons that enter the detector parallel to the planes and travel a long way through a single plane before scattering into the detector or bending sharply in the magnetic field. These events have a number of characteristic topologies that can be used to separate the signal and background. The events typically contain several hits above the muon 


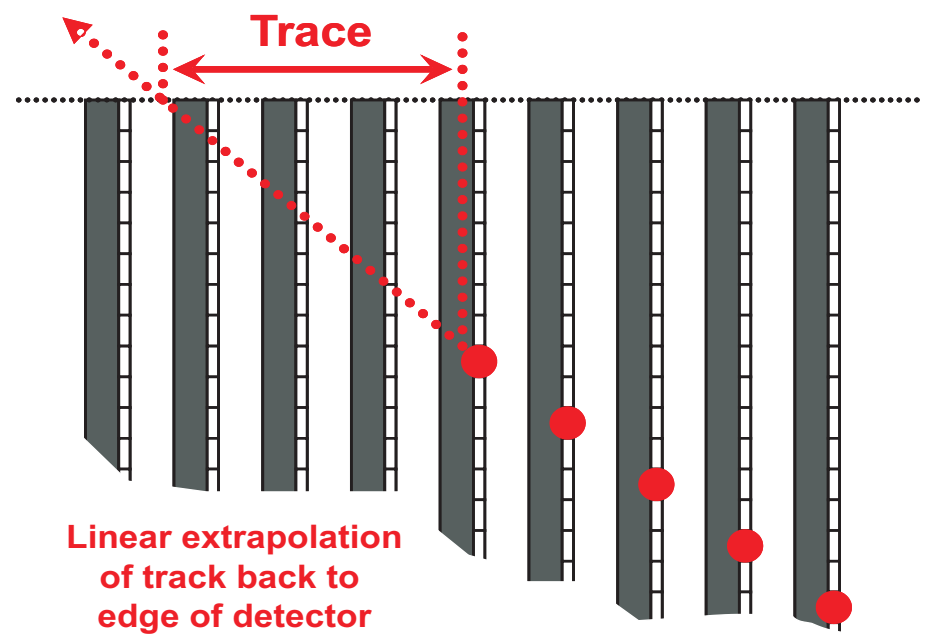

Figure 8.8: The majority of contained cosmic muons are removed by applying a trace cut. The muon track is projected back to the edge of the detector to determine the muon entry point in the detector. The trace is given by the horizontal distance between the track vertex and muon entry point.

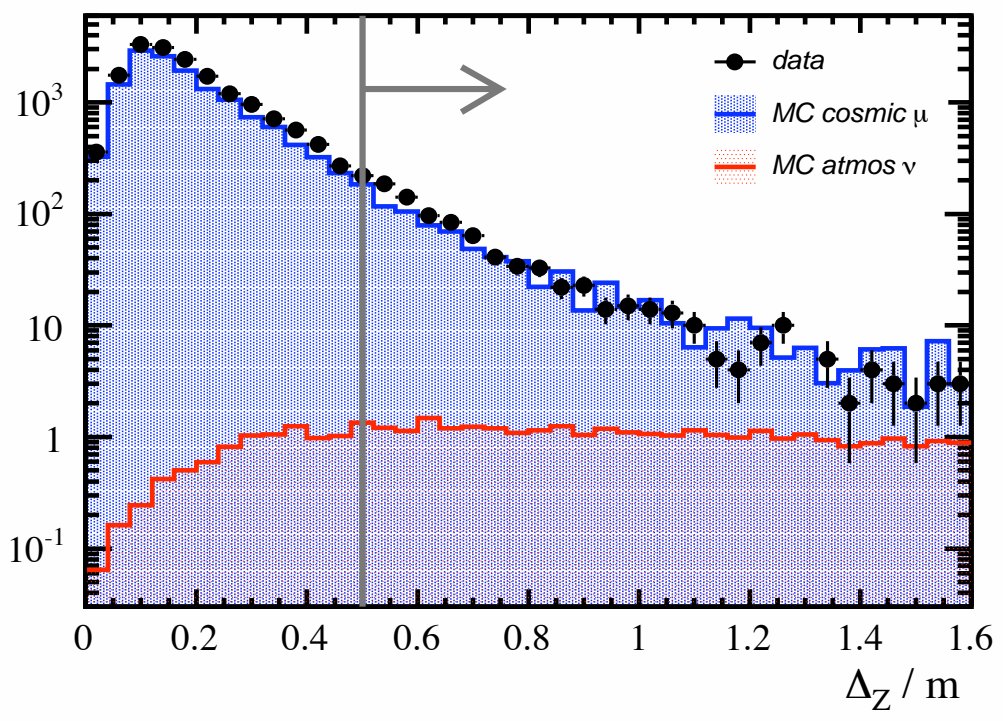

Figure 8.9: Distribution of $\Delta_{Z}$ for $F C / P C D N$ events that pass the track selection cuts. The data are represented by the black points; $M C$ cosmic muons are represented by the blue histogram; and MC atmospheric $\nu_{\mu} / \bar{\nu}_{\mu}$ $C C$ events are represented by the red histogram. 
(a)

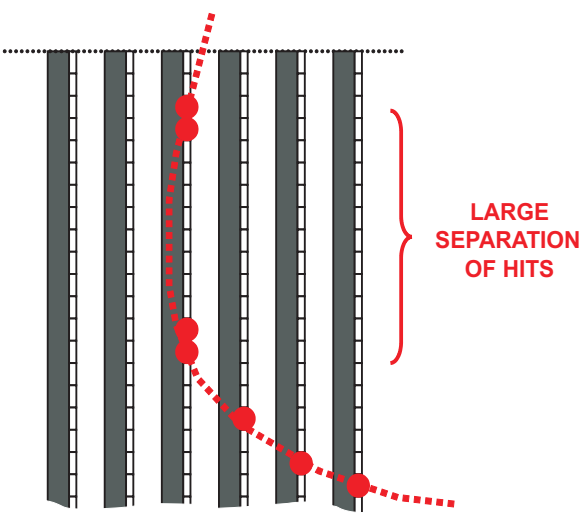

(b)

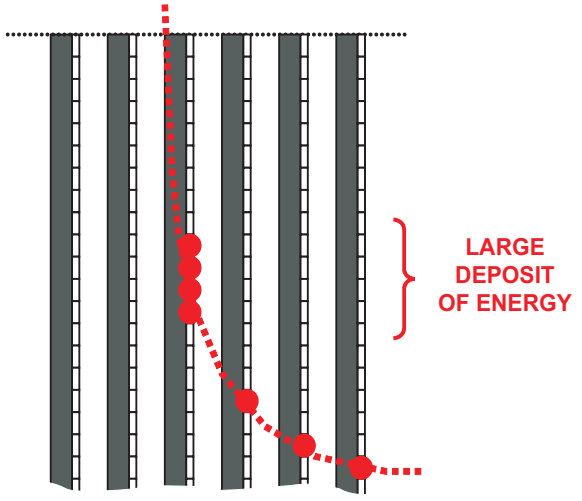

Figure 8.10: The cosmic muon background can be identified using characteristic topologies. The events typically contain several early hits above the reconstructed track or large energy deposits in the early planes.

vertex or large energy deposits around the muon vertex; events may also reverse their direction along the Z-axis and deposit energy in two different positions in some planes (see Figure 8.10). The background is reduced by analysing the energy deposits and event topology around the muon vertex. The charge-weighted mean $\left\langle\Delta_{T}\right\rangle$ and $r m s$ displacement $\left\langle\Delta_{T}^{2}\right\rangle^{\frac{1}{2}}$ of strips from the muon vertex is calculated for the $\mathrm{U}$ and $\mathrm{V}$ views in the region \pm 4 planes from the vertex. Events are required to satisfy: $\left\langle\Delta_{U}\right\rangle<0.25 \mathrm{~m},\left\langle\Delta_{U}^{2}\right\rangle^{\frac{1}{2}}<0.5 \mathrm{~m},\left\langle\Delta_{V}\right\rangle<0.25 \mathrm{~m},\left\langle\Delta_{V}^{2}\right\rangle^{\frac{1}{2}}<0.5 \mathrm{~m}$. The 3D displacement $\Delta_{R}$ of strips from the muon vertex is also calculated in the region \pm 4 planes from the vertex by joining together the $\mathrm{U}$ and $\mathrm{V}$ strips in adjacent planes. The maximum 3D displacement $\Delta_{R}^{\max }$ is required to satisfy $\Delta_{R}^{\max }<1.25 \mathrm{~m}$. Finally the total pulse height in each plane is calculated in the region \pm 4 planes from the vertex. The maximum pulse height $Q_{\max }$ in a plane is required to satisfy $Q_{\max }<300$ PEs. The distributions of these four topology variables are shown for data, MC cosmic muons and MC atmospheric $\nu_{\mu} / \bar{\nu}_{\mu} \mathrm{CC}$ events in Figures 8.11 (a)-(d) for FC events and Figures 8.12 (a)-(d) for PCDN events. There is good agreement between data and MC, and good separation between signal and background. The topology cuts remove approximately $70 \%$ of cosmic muons and approximately $10 \%$ of atmospheric neutrinos, reducing the signal-to-background ratio to $1: 3$ for both $\mathrm{FC}$ and PCDN events. 
(a)

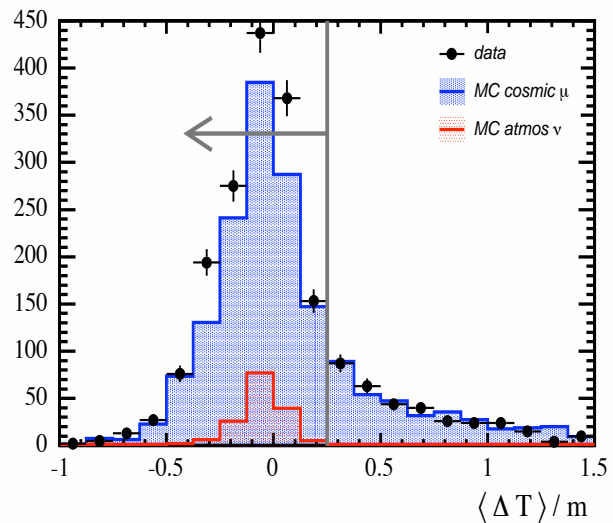

(c)

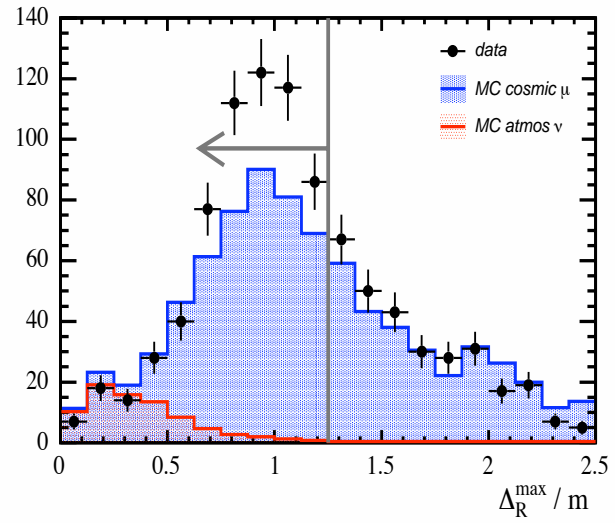

(b)

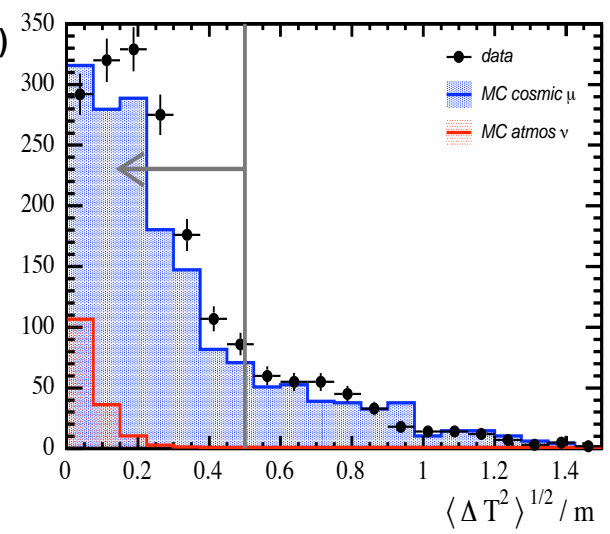

(d)

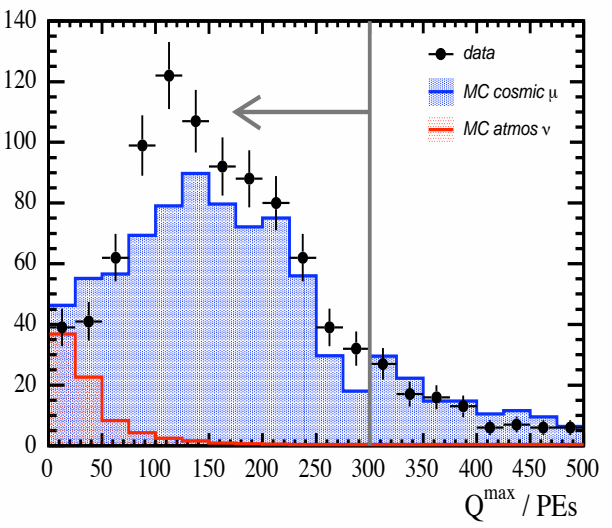

Figure 8.11: Distributions of topology variables used to select $F C$ events following trace cut. (a) mean displacement of hits around vertex. (b) rms displacement of hits around vertex. (c) maximum $3 D$ displacement of hits around vertex. (d) maximum pulse height around vertex. 
(a)

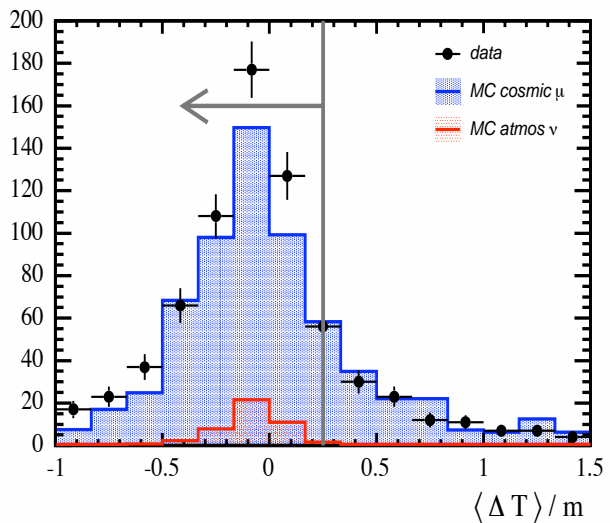

(c)

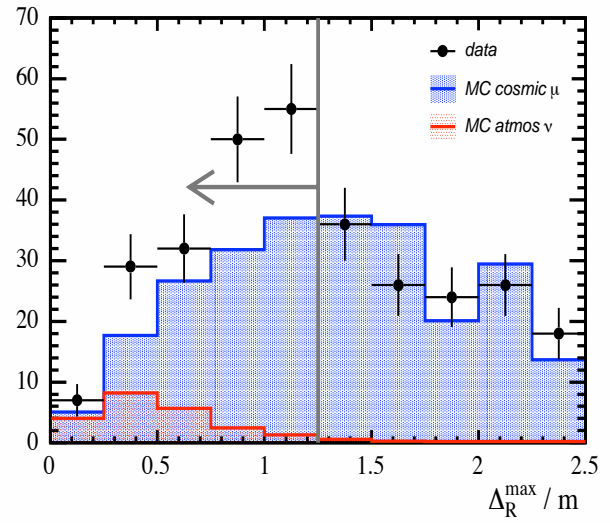

(b)

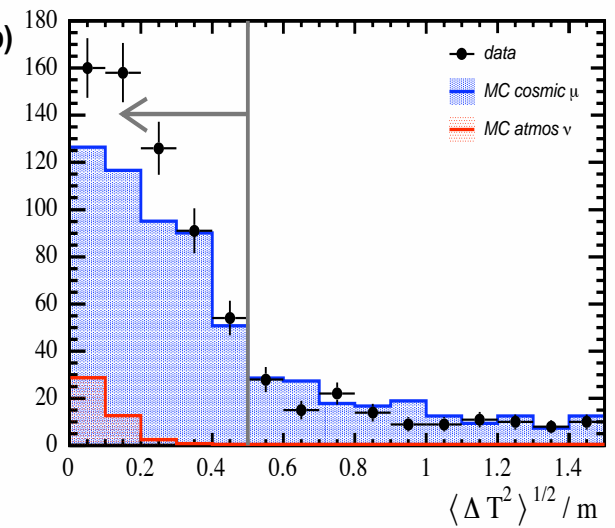

(d)

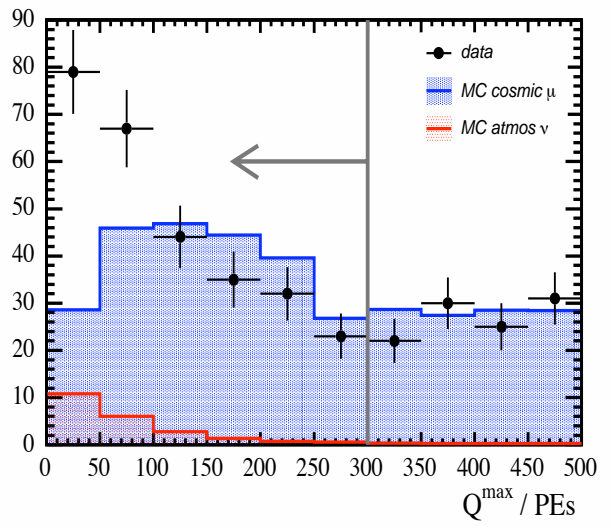

Figure 8.12: Distributions of topology variables used to select PCDN events following trace cut. (a) mean displacement of hits around vertex. (b) rms displacement of hits around vertex. (c) maximum 3D displacement of hits around vertex. (d) maximum pulse height around vertex. 

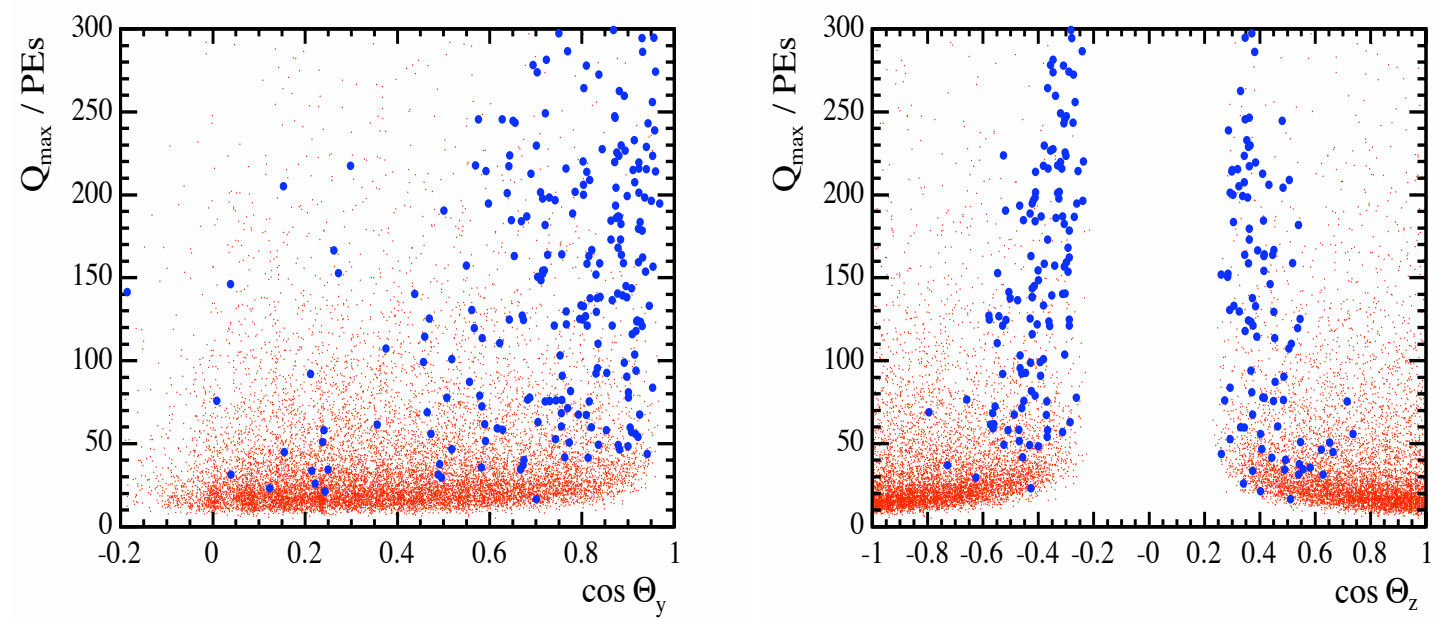

Figure 8.13: Distribution of vertex charge $Q_{\max }$ as a function of $\cos \Theta_{y}$ (left) and $\cos \Theta_{z}$ (right), the components of the track direction along the $Y$-axis and Z-axis at the upper track vertex. The red points represent $M C$ atmospheric neutrinos, and the blue points represent MC cosmic muons.

- Vertex Charge : The remaining background is reduced by varying the $Q_{\max }$ cut with the direction of the muon track. Figure 8.13 shows the distribution of $Q_{\max }$ as a function of $\cos \Theta_{y}$ and $\cos \Theta_{z}$, the components of the track direction along the Y-axis and Z-axis at the upper track vertex. The cosmic muon background is characterized by large energy deposits at the vertex and directions peaked towards vertical angles and small angles to the planes. In addition more than $90 \%$ of the remaining cosmic muons are found to span less than 20 planes. In contrast, the atmospheric neutrino signal is characterized by smaller energy deposits at the vertex and a more uniform angular distribution. In order to separate the signal and background, the events spanning less than 20 planes with $\cos \Theta_{y}>$ 0.7 or $\left|\cos \Theta_{z}\right|<0.5$ are required to satisfy an additional cut of $Q_{\max }<100$ PEs. The $Q_{\max }$ distribution for these events is shown for data, MC cosmic muons and MC atmospheric $\nu_{\mu} / \bar{\nu}_{\mu}$ CC events in Figure 8.14 for FC events and Figure 8.15 for PCDN events. The additional $Q_{\max }$ cut removes approximately $50 \%$ of cosmic muons and approximately $2 \%$ of atmospheric neutrinos, giving a signal-tobackground ratio of $1: 1.3$ for FC events and $1: 1.4$ for PCDN events. 
(a)

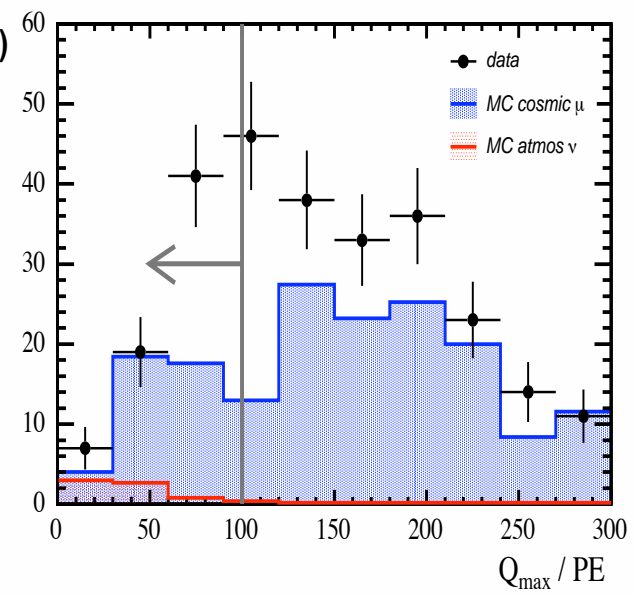

(b)

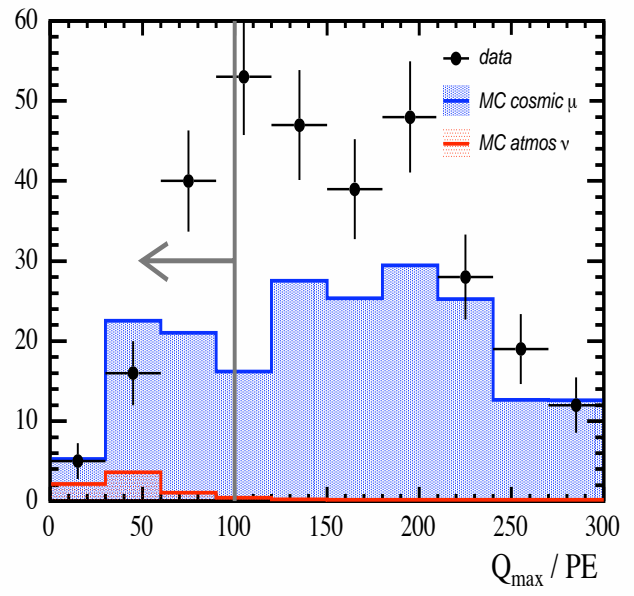

Figure 8.14: Distribution of vertex charge $Q_{\max }$ for $F C$ tracks with less than 20 planes which pass the trace and topology cuts. This is shown for: (a) track directions of $\cos \Theta_{y}>0.7$; (b) track directions of $\left|\cos \Theta_{z}\right|<0.5$.

(a)

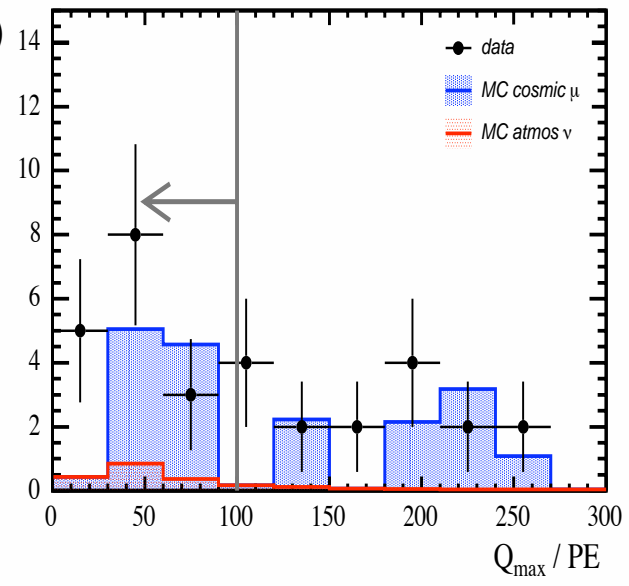

(b)

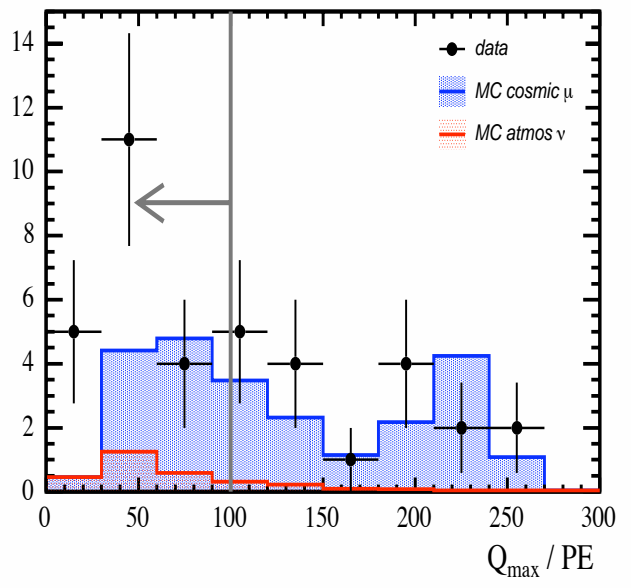

Figure 8.15: Distribution of vertex charge $Q_{\max }$ for PCDN tracks with less than 20 planes which pass the trace and topology cuts. This is shown for: (a) track directions of $\cos \Theta_{y}>0.7$; (b) track directions of $\left|\cos \Theta_{z}\right|<0.5$. 


\subsubsection{Veto Shield Cuts}

The containment cuts select 159 FC events and 33 PCDN events from the data set. This compares with a $\mathrm{MC}$ expectation of $98 \pm 14 \mathrm{FC}$ events and $23 \pm 6$ PCDN events for the cosmic muon background, and $72.3 \pm 14.4$ FC events and 16.6 \pm 3.3 PCDN events for the atmospheric $\nu_{\mu} / \bar{\nu}_{\mu}$ CC signal. Once the cosmic muon background has been reduced to a level similar to the expected atmospheric $\nu_{\mu} / \bar{\nu}_{\mu} \mathrm{CC}$ signal, the veto shield is used to bring the background down to a low level.

An event is vetoed if any activity is observed in the section of shicld above the muon vertex in a window of \pm 100 ns around the vertex time. If the vertex occurs less than $1 \mathrm{~m}$ from the end of a shield section, the activity in the two adjacent sections above the track is used to veto events. The accuracy of the shicld timing information is improved by applying calibration corrections to the measured shield times. The shield modules and detector planes share common readout electronics at the VARC level. This is the level at which the largest offsets occur in the timing system. Therefore the VARC time offsets calculated for the detector timing calibration (see Section 5.2) are used to provide an approximate shicld timing calibration. Timing corrections are also applied to account for the propagation of signals along the readout fibres. Figure 8.16 shows the distribution of the differences in time between the muon vertex and associated hits in the shicld for stopping cosmic muons and selected FC/PCDN cvents. In both cases the associated shield hits are contained in a peak within the \pm 100 ns window.

The efficiency for tagging cosmic muons with the veto shield is measured using stopping cosmic muons selected from the data as described in Section 7.4.1. The shicld efficiency is defined as the percentage of selected events tagged by the above shield cuts. Table 8.1 lists the efficiencies calculated for each section of the shicld. The efficiency is similar in all the sections, but slightly lower in section 3 due to a high noise rate. The overall efficiency is calculated to be $97.1 \pm 0.1 \%$. A second measurement of the shield efficiency is performed using the sclected FC and PCDN events. The trace, topology and vertex charge cuts are all removed, giving a sample of 33,000 events with an expected signal of 110 events. The shield efficiency is calculated using the event sample and a correction of $+0.2 \%$ is applied to account for signal cvents. Table 8.1 lists the efficiencies calculated for each section of the shield. The overall efficiency is calculated to be $97.2 \pm 0.1 \%$. The two different methods of calculating the shield efficiency produce results that are in good agreement. 
(a)

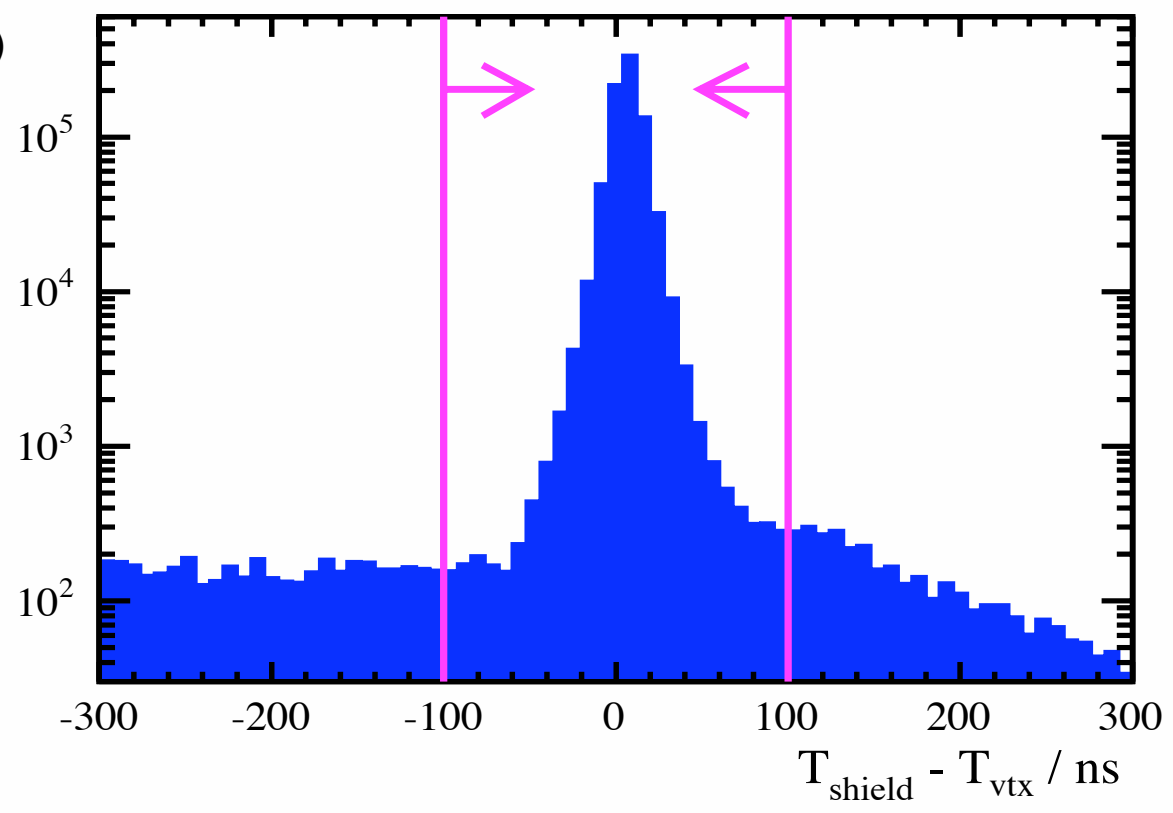

(b)

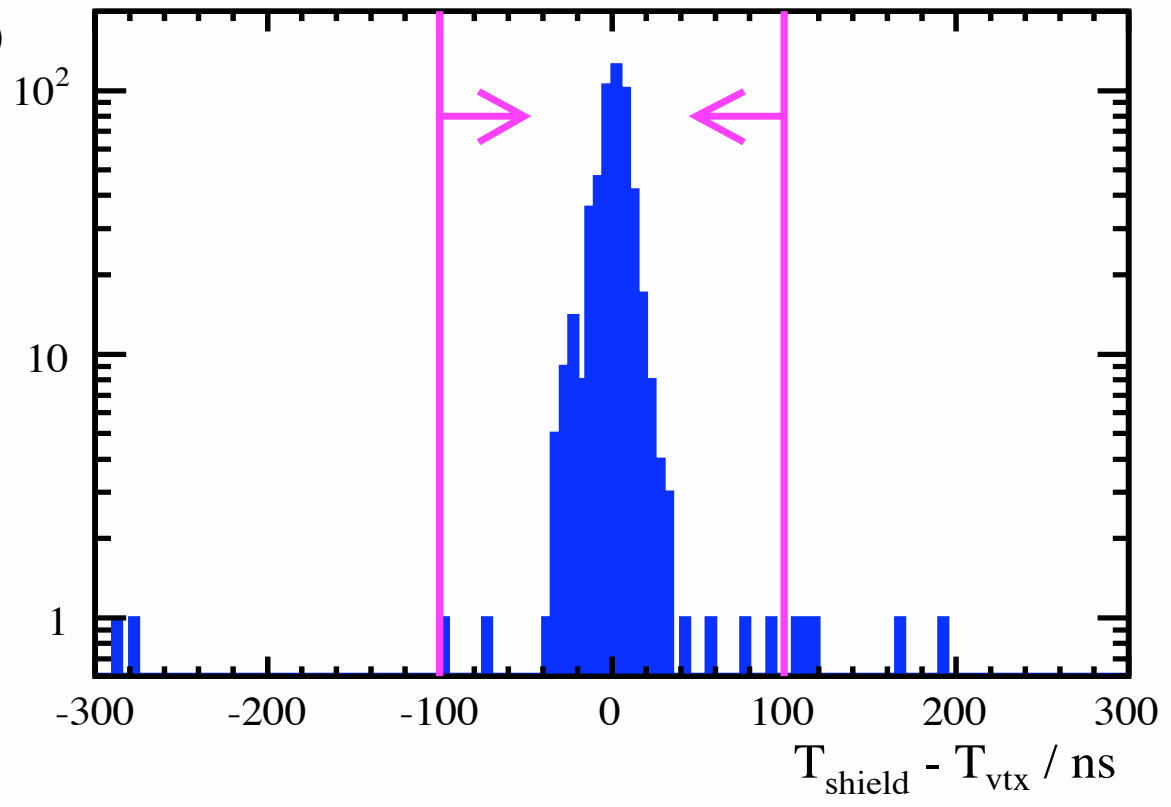

Figure 8.16: Time difference between shield hits and muon vertex times for (a) stopping muons (b) events which pass FC and down-going PC selection. The associated shield hits are contained in a narrow peak well inside the \pm 100 ns timing window. 


\begin{tabular}{|c|c|c|}
\hline & \multicolumn{2}{|c|}{ Cosmic Muon Vetoing Rate } \\
\hline & (stopping muons) & (FC/PCDN events) \\
\hline Section 1 & $97.2 \pm 0.2 \%$ & $97.1 \pm 0.2 \%$ \\
\hline Section 2 & $97.4 \pm 0.2 \%$ & $97.4 \pm 0.2 \%$ \\
\hline Section 3 & $96.6 \pm 0.2 \%$ & $96.8 \pm 0.2 \%$ \\
\hline Section 4 & $97.1 \pm 0.2 \%$ & $97.3 \pm 0.2 \%$ \\
\hline Average & $97.1 \pm 0.1 \%$ & $97.2 \pm 0.1 \%$ \\
\hline
\end{tabular}

Table 8.1: Shield efficiencies calculated for each section of the shield. The first column lists the efficiencies calculated using the stopping muon sample. The second column lists the efficiencies calculated using the $F C / P C D N$ event sample with a correction of $+0.2 \%$ applied to account for the expected atmospheric neutrino signal within the sample.

\begin{tabular}{|c|c|}
\hline & Accidental Signal Tagging Rate \\
\hline Section 1 & $2.1 \pm 0.2 \%$ \\
Section 2 & $2.1 \pm 0.2 \%$ \\
Scction 3 & $2.3 \pm 0.2 \%$ \\
Scction 4 & $2.2 \pm 0.2 \%$ \\
\hline Average & $2.2 \pm 0.1 \%$ \\
\hline
\end{tabular}

Table 8.2: Signal losses for each section of the shield due to accidental coincidences with shield activity generated by noise or natural radioactivity. 


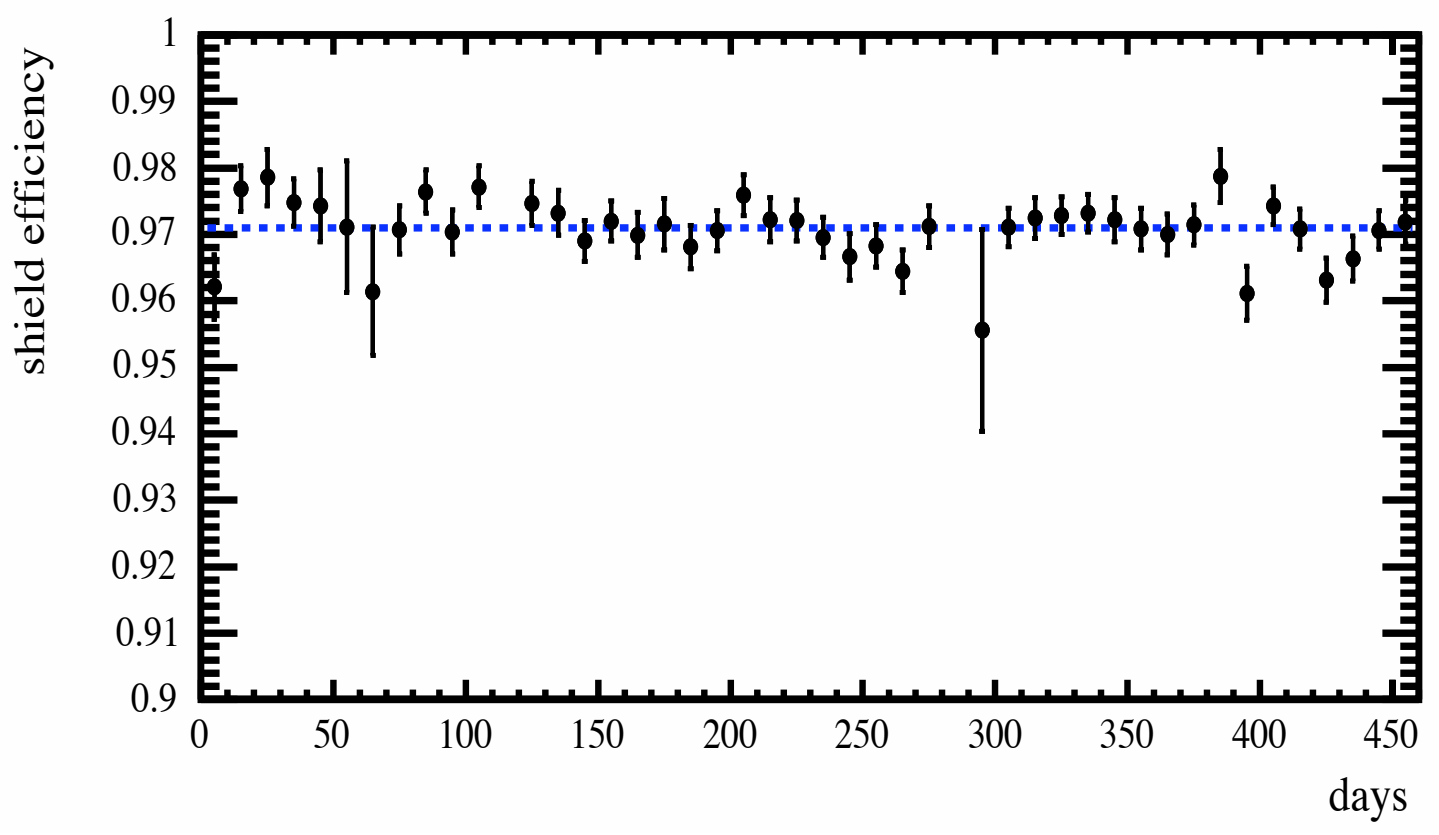

Figure 8.17: Shield efficiency as a function time for data acquired between 1st August 2003 and 31st October 2004. The data is divided into 2-week intervals and the shield efficiency is calculated for each interval using stopping muons. The blue line indicates the mean shield efficiency of $97.1 \%$. The number of days is defined relative to 1st August 2003. 
In order to study the long term performance of the veto shield, the data are divided into 2-weck intervals and the shicld efficiency is calculated for cach interval using the sample of stopping muons. Figure 8.17 shows the shield efficiency as a function of time for data acquired between 1st August 2003 and 31st October 2004. The shicld efficiency is found to remain constant over time to within $1 \%$.

A small percentage of signal events are vetoed by the shield due to accidental coincidence with shicld activity generated by natural radioactivity or noise. The loss of signal due to this accidental tagging is measured by combining a sample of MC atmospheric $\nu_{\mu} / \bar{\nu}_{\mu}$ CC events with shield hits extracted from the pre-trigger window of events in the data. Table 8.2 lists the loss of signal calculated for each section of the shicld. The overall loss of signal is calculated to be $2.2 \pm 0.1 \%$.

The shield cuts are applied to the selected FC/PCDN events and are found to veto 103 FC events and 17 PCDN events. Applying the measured shicld efficiency to the MC cosmic muon background, the expected number of vetoed events is calculated to be $95 \pm 14$ FC cvents and $22 \pm 6$ PCDN cvents. The obscrved number of vetoed events is in agreement with the MC expectation. The vetoed events are compared with the selected MC cosmic muon events to test the agreement between data and MC. Figure 8.18 shows the distribution of the distance between the muon vertex and the nearest detector edge for data and MC. The distributions are peaked around $1.5 \mathrm{~m}$, consistent with cosmic muons that have entered through the side of the detector. Figure 8.19 shows the distribution of the reconstructed muon zenith angle for data and MC. The distributions are peaked towards vertical angles, consistent with the cosmic muon background. 


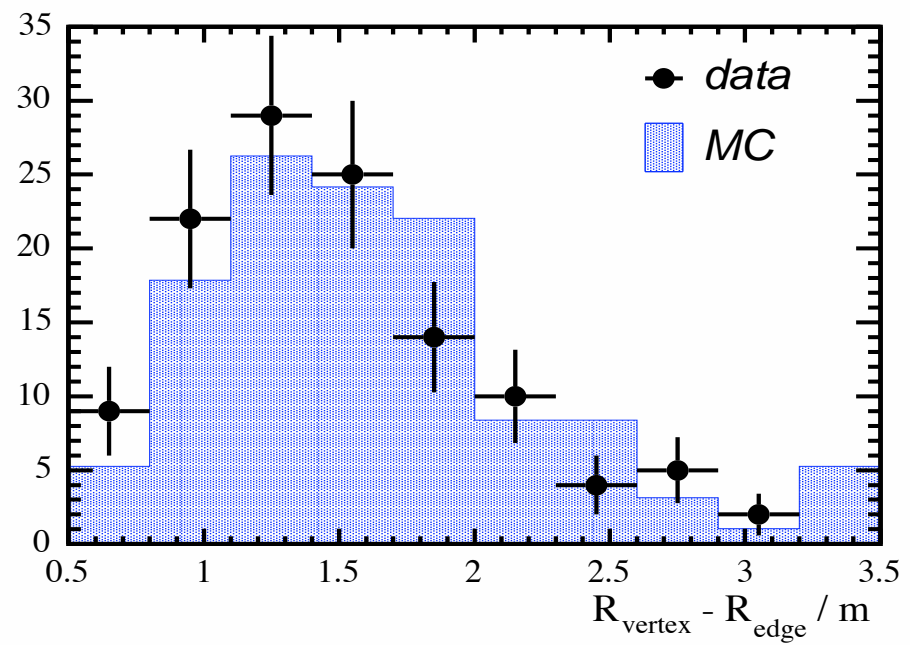

Figure 8.18: Distribution of distances between track vertex and nearest detector edge for events which pass the containment cuts. The events in data that are tagged by the veto shield are represented by the black points, $M C$ cosmic muons are represented by the blue histogram.

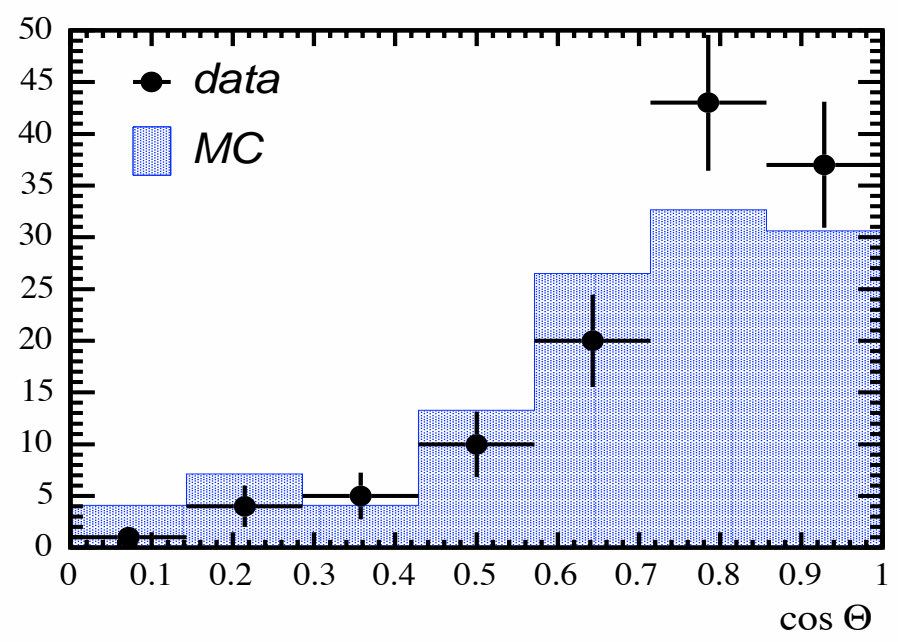

Figure 8.19: Distribution of reconstructed muon zenith angle for events which pass the containment cuts. The events in data that are tagged by the veto shield are represented by the black points, $M C$ cosmic muons are represented by the blue histogram. 


\subsubsection{Results}

The numbers of events selected after each stage of the FC and PCDN selection are shown in Table 8.3. The crrors quoted for MC cosmic muons are calculated by combining the statistical error in quadrature with a $10 \%$ systematic error in the normalization; the errors quoted for the MC atmospheric neutrinos are given by a $20 \%$ systematic error in the normalization (sec Section 8.9). The data and $\mathrm{MC}$ are in found to be in reasonable agreement throughout the event selection, although there is an excess of data in the carly stages. After applying the veto shicld, 56 FC cvents and 16 PCDN events are selected from the data. This compares with a MC expectation of: $2.8 \pm 0.4 \mathrm{FC}$ events and $0.7 \pm 0.3$ PCDN events for cosmic muons; $70.7 \pm 14.1 \mathrm{FC}$ events and $16.6 \pm 3.3$ PCDN cvents for atmospheric $\nu_{\mu} / \bar{\nu}_{\mu}$ CC cvents; $3.8 \pm 0.8 \mathrm{FC}$ cvents and $<0.1 \mathrm{PCDN}$ events for atmospheric $\nu_{e} / \bar{\nu}_{e} \mathrm{CC}$ and $\mathrm{NC}$ events.

The cosmic muon background that remains following the veto shield cuts can be measured directly from the data by applying the known efficiencies for vetoing signal and background to the number of vetoed events. The total number of signal $(S)$ and background $(B)$ events in the data can be related to the total number of vetoed $(V)$ and sclected $(N)$ events as follows:

$$
\begin{gathered}
N=\left(1-\eta_{B}\right) B+\eta_{S} S \\
V=\eta_{B} B+\left(1-\eta_{S}\right) S
\end{gathered}
$$

where:

$$
\begin{aligned}
& \eta_{B}=\text { efficiency for vetoing cosmic muon background }=97.1 \% \\
& \eta_{S}=\text { cfficiency for selecting atmospheric neutrino signal }=97.8 \%
\end{aligned}
$$

Substituting $N=72$ cvents and $V=120$ events into Equation 8.1 gives an expectation of $\left(1-\eta_{B}\right) B=3.5 \pm 0.3$ events for the cosmic muon background that remains in the selected FC/PCDN events. This is in good agreement with the MC expectation of $3.5 \pm 0.5$ events for the cosmic muon background, and is also small compared with the $\mathrm{MC}$ expectation of $86.9 \pm 17.3$ events for the atmospheric $\nu_{\mu} / \bar{\nu}_{\mu} \mathrm{CC}$ signal. The FC/PCDN events that have been vetoed by the shield are used to represent the cosmic muon background in the atmospheric neutrino oscillation analysis. 


\begin{tabular}{|c|c|c|c|c|}
\hline FC & Data & \multicolumn{3}{|c|}{ MC Expectation } \\
Cuts & & cosmic muons & $\nu_{\mu} / \bar{\nu}_{\mu} \mathrm{CC}$ & $\nu_{e} / \bar{\nu}_{e} \mathrm{CC}, \mathrm{NC}$ \\
\hline Track Q11ality & 18300 & $13680 \pm 1370$ & $86.9 \pm 17.3$ & $4.9 \pm 1.0$ \\
Trace & 961 & $760 \pm 81$ & $77.2 \pm 15.4$ & $4.7 \pm 0.9$ \\
Topology & 400 & $245 \pm 29$ & $73.3 \pm 14.7$ & $4.0 \pm 0.8$ \\
Vertex Charge & 159 & $98 \pm 14$ & $72.3 \pm 14.5$ & $3.9 \pm 0.8$ \\
Veto Shield & 56 & $2.8 \pm 0.4$ & $70.7 \pm 14.1$ & $3.8 \pm 0.8$ \\
\hline Total & 56 & \multicolumn{3}{|c|}{$77.3 \pm 14.9$} \\
\hline
\end{tabular}

\begin{tabular}{|c|c|c|c|c|}
\hline PCDN & Data & \multicolumn{3}{|c|}{ MC Expectation } \\
Cuts & & cosmic muons & $\nu_{\mu} / \bar{\nu}_{\mu}$ CC & $\nu_{e} / \bar{\nu}_{e} \mathrm{CC}, \mathrm{NC}$ \\
\hline Track Quality & 16360 & $13770 \pm 1380$ & $25.0 \pm 5.0$ & $0.3 \pm 0.0$ \\
Track Length & 14300 & $12040 \pm 1210$ & $21.5 \pm 4.3$ & $0.2 \pm 0.0$ \\
Trace & 245 & $237 \pm 28$ & $19.9 \pm 4.0$ & $0.2 \pm 0.0$ \\
Topology & 47 & $29 \pm 7$ & $17.3 \pm 3.4$ & $0.1 \pm 0.0$ \\
Vertex Charge & 33 & $23 \pm 6$ & $16.6 \pm 3.3$ & $0.1 \pm 0.0$ \\
Veto Shicld & 16 & $0.7 \pm 0.3$ & $16.2 \pm 3.2$ & $<0.1$ \\
\hline Total & 16 & \multicolumn{3}{|c|}{$16.9 \pm 3.2$} \\
\hline
\end{tabular}

Table 8.3: The number of events selected at each stage of the $F C$ and $P C D N$ event selection. The expected signal is calculated for no oscillations. The measured shield efficiencies are applied to the $M C$ cosmic muons and $M C$ atmospheric neutrinos to obtain the final $M C$ expectation. The errors quoted for $M C$ cosmic muons are calculated by combining the statistical error in quadrature with a $10 \%$ systematic error in the normalization; the errors quoted for the $M C$ atmospheric neutrinos are given by a $20 \%$ systematic error in the normalization. 


\subsection{Selection of Up-Going PC Events}

\subsubsection{Topology Cuts}

The primary tool used to separate the PCUP signal from the cosmic muon background is timing information. The event direction is determined by analysing the measured times along the muon track. In order to determine the event direction accurately from timing information, the muon track must contain a sufficient number of timing measurements and must be well reconstructed. The following topology cuts are applied to select wellmeasured muon tracks:

- Track Length : For short cosmic muon tracks, the muon direction may be misreconstructed as up-going due to the small number of timing measurements or errors in the reconstruction. Therefore an additional track length cut is placed on PCLP events. Tracks are required to be $\geq 10$ planes and $>1 \mathrm{~m}$ in length. This ensures that the timing is sampled a sufficient number of times along the track to enable an accurate determination of the direction, and that the range of the track is long enough to separate the start and end of the track within the timing resolution of the detector.

- Track Topology : For cosmic muons that enter the detector parallel to the scintillator strips or bend sharply in the magnetic field, errors in the reconstruction of events can generate scatter in the timing at the beginning of the track or can cause the start of the track to be reconstructed below the end of the track. This can cause events to be mis-reconstructed as up-going. In order to reduce this background, two of the topology cuts used in the FC/PCDN event selection are also applied to the PCLP events. Events are required to satisfy $\Delta_{R}^{\max }<1.25$ and $Q_{\max }<300$ PEs. This removes the background events that contain scatter in the timing due to the event topology.

The track length cuts remove approximately $70 \%$ of cosmic muons and approximatcly $15 \%$ of atmospheric neutrinos. The track topology cuts then remove approximately $10 \%$ of cosmic muons and approximately $5 \%$ of atmospheric neutrinos. The overall effect of the cuts is to reduce the signal-to-background ratio to $1: 12$. 


\subsubsection{Timing Cuts}

The PCUP signal is separated from the background by applying a series of timing cuts. The main background arises from stopping cosmic muons that are mis-reconstructed as up-going from timing information. This background is reduced by analysing the timing information and applying cuts to ensure that events are correctly reconstructed as up-going. The following timing cuts are applied to select PCUP events:

- RMS cuts : The event direction is determined as described in Section 7.4.1. Timing fits are applied to the measured times and distances along each track with fixed gradients $\beta=+1$ (down-going) and $\beta=-1$ (up-going). The rms deviations $\left(r m s_{\text {down }}\right.$ and $\left.r m s_{u p}\right)$ are calculated for each fit. Timing cuts are applied to the values of $r m s_{u p}, r m s_{d o w n}$ and $r m s_{u p}-r m s_{\text {down }}$ to cnsure that the up-going fit is significantly better than the down-going fit. Figure 8.20 shows the distribution of $r m s_{\text {up }}$ vs $r m s_{\text {down }}$ for events that satisfy the topology cuts. The cosmic muon background is clustered close to $r m s_{u p}-r m s_{\text {down }}=0 \mathrm{~ns}$. In contrast, the atmospheric neutrino signal is distributed around $r m s_{u p}=2.5 \mathrm{~ns}$, consistent with the timing resolution of the detector. Figures 8.21 (a)-(c) show the distribution of $r m s_{u p}, r m s_{d o w n}$ and $r m s_{u p}-r m s_{\text {down }}$ for data, $\mathrm{MC}$ cosmic muons and $\mathrm{MC}$ atmospheric $\nu_{\mu} / \bar{\nu}_{\mu} \mathrm{CC}$ events. The distributions all show reasonable agreement between data and MC. Events are required to satisfy: $r m s_{u p}<4.33$ ns to select up-going fits with small timing scatter, $r m s_{\text {down }}>3.33$ ns to select down-going fits with large timing scatter, and $r m s_{u p}-r m s_{\text {down }}<-1.66 \mathrm{~ns}$, to select events where the up-going fit is significantly better than the down-going fit. The rms cuts remove approximately $90 \%$ of MC cosmic muons, and approximately $5 \%$ of $\mathrm{MC}$ atmospheric neutrinos that satisfy the topology cuts.

- RMS/range : The ratio between the $r m s$ deviation for the up-going timing fit and the range of the track provides an additional means of discriminating between up-going and down-going events. If an up-going timing fit is applied to a perfectly measured down-going track, this ratio is given by $c * r m s /$ range $=1 / \sqrt{3} \approx 0.58$. Figure 8.22 shows the distribution of $c * r m s / r a n g e$ for data, MC cosmic muons and $\mathrm{MC}$ atmospheric $\nu_{\mu} / \bar{\nu}_{\mu} \mathrm{CC}$ events. The cosmic muon background has a broad peak around $c * r m s /$ range $=0.6$, consistent with down-going events that have been mis-reconstructed as up-going. In contrast, the atmospheric neutrino signal 


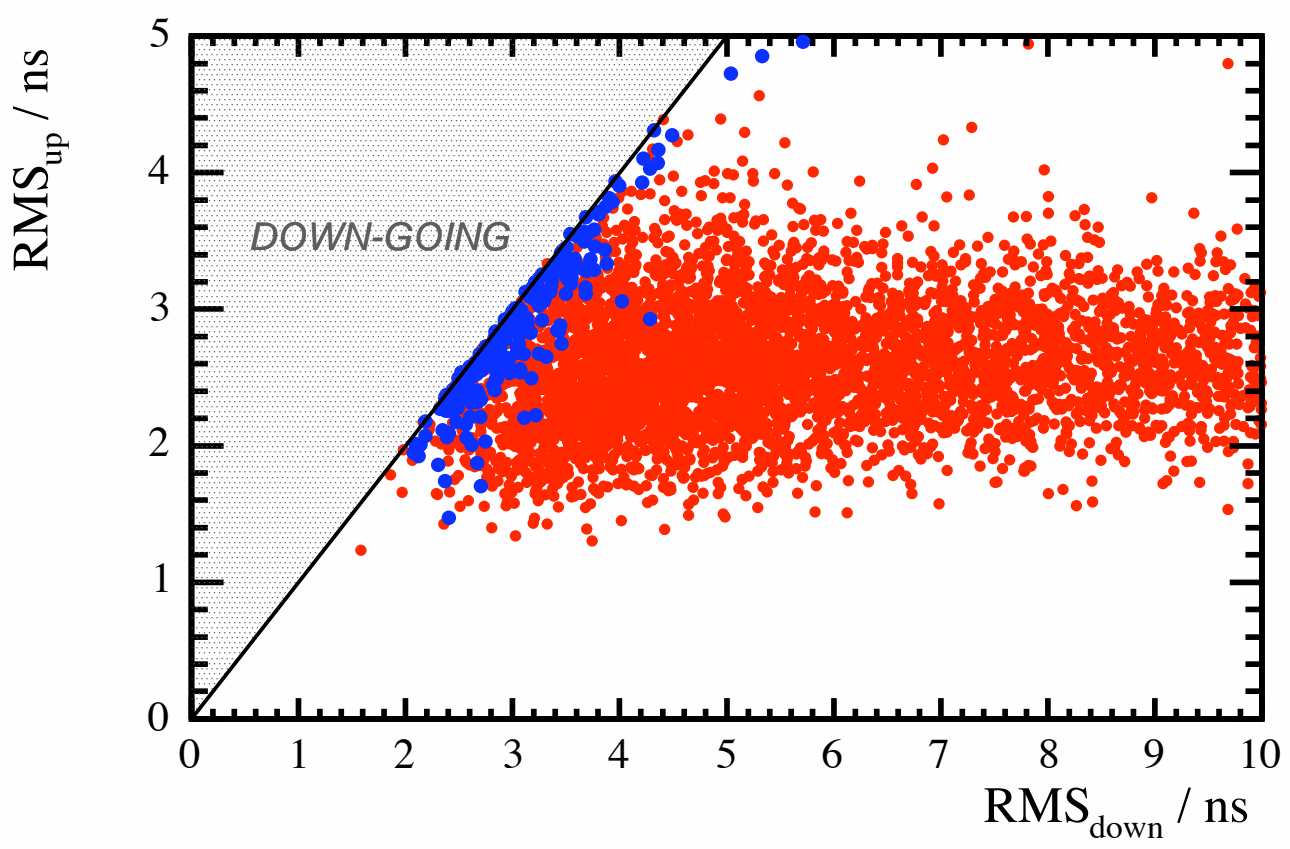

Figure 8.20: Distribution of $r m s_{\text {up }}$ vs $r m s_{\text {down }}$ for PCUP events which pass the topology cuts. The red points represent $M C$ atmospheric $\nu_{\mu} / \bar{\nu}_{\mu}$ $C C$ events and the blue points represent $M C$ cosmic muons.

is peaked at $c * r m s /$ range $<0.3$, consistent with events that have been correctly reconstructed as up-going. Events are required to satisfy $c * r m s /$ range $<0.66$. The rms/range cut removes approximately $35 \%$ of $\mathrm{MC}$ cosmic muons, and approximately $15 \%$ of MC atmospheric neutrinos that satisfy the topology cuts.

- Time Slope : An additional timing fit is applied with the constraint on the muon velocity removed. This fit provides a measurement of the reciprocal muon velocity $1 / \beta$. The sign of $1 / \beta$ is required to correspond to an up-going muon. Figure 8.23 shows the distribution of $1 / \beta$ for data, MC cosmic muons and MC atmospheric $\nu_{\mu} / \bar{\nu}_{\mu}$ CC events. The atmospheric neutrino signal is peaked at -1 , consistent with muons travelling upwards at the speed of light. In contrast, the cosmic muon background is peaked around $1 / \beta=0$. Events are required to satisfy $1 / \beta<-0.5$. The $1 / \beta$ cut removes approximately $75 \%$ of MC cosmic muons, and approximately $5 \%$ of MC atmospheric neutrinos that satisfy the topology cuts. 
(a)

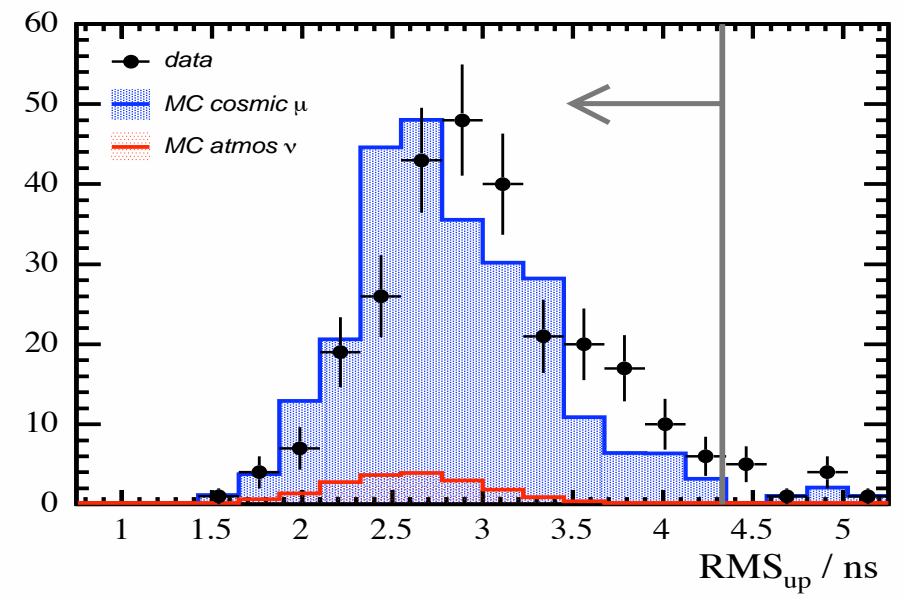

(b)

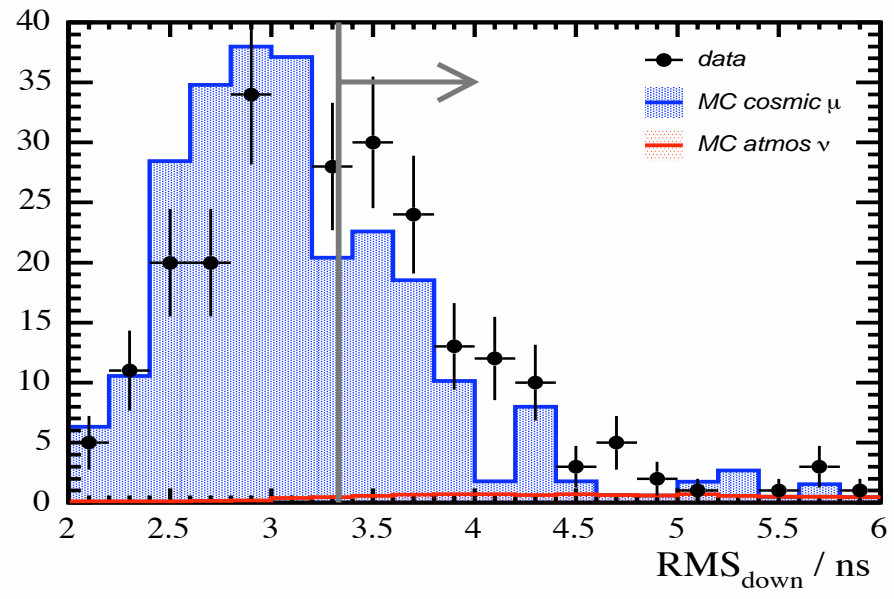

(c)

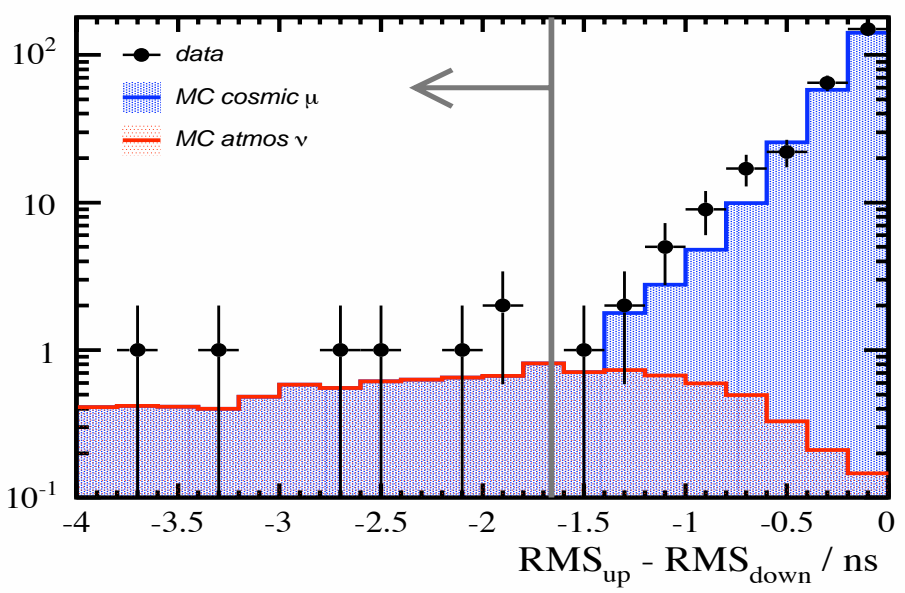

Figure 8.21: Distributions of rms timing variables used to select up-going events shown for events which satisfy the topology cuts. (a) rms deviations of up-going timing fits to muon tracks. (b) rms deviations of down-going timing fits to muon tracks. (c) difference between the rms deviations of the up-going and down-going timing fits to muon tracks. 


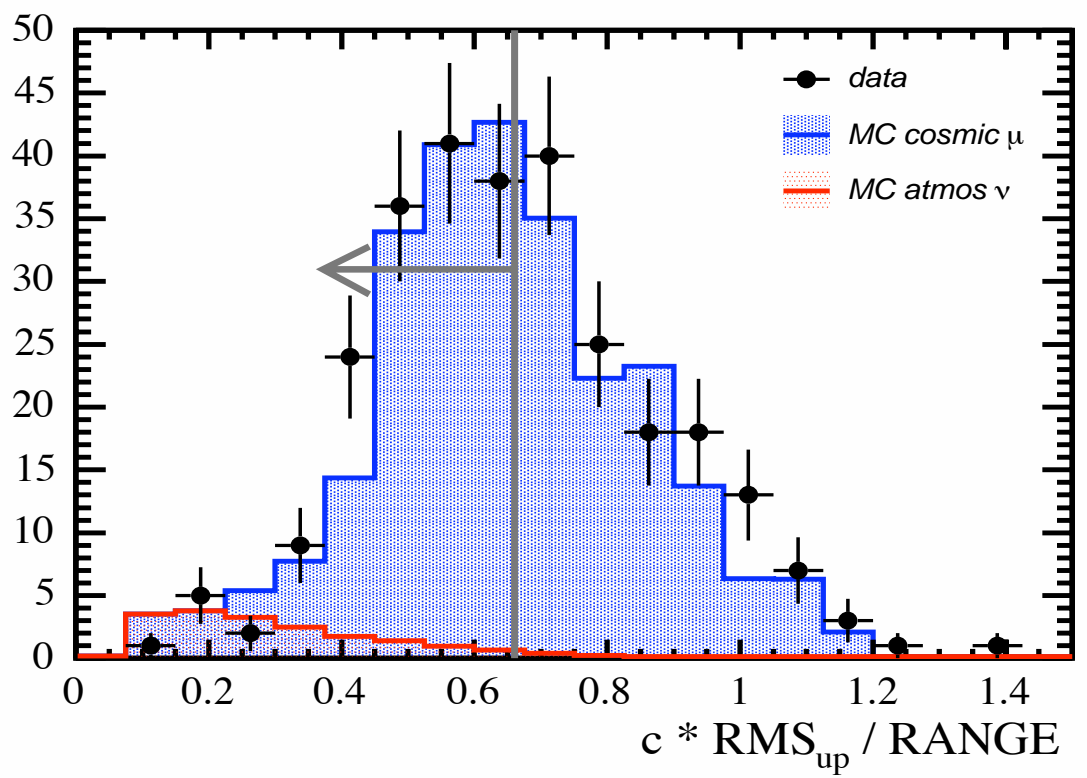

Figure 8.22: Distribution of the rms deviation for the up-going timing fit over the track range for events which satisfy the topology cuts.

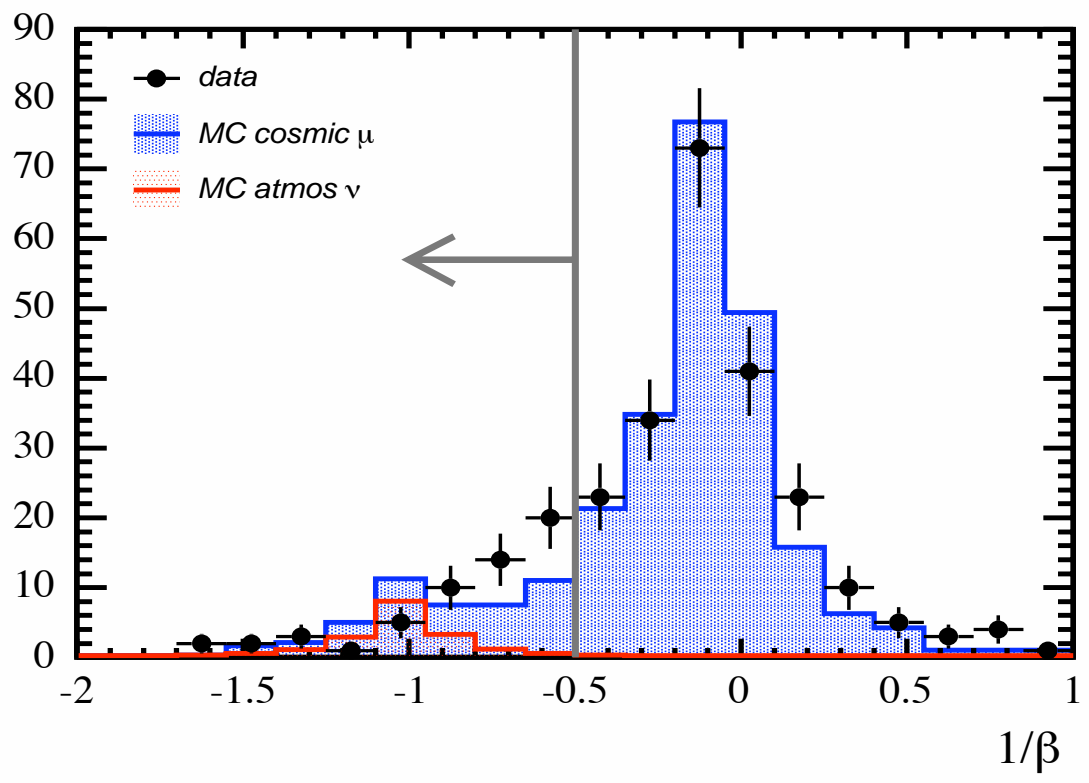

Figure 8.23: Distribution of reciprocal velocity $1 / \beta$ for events which satisfy the topology cuts. Negative values of $1 / \beta$ correspond to up-going events, positive values of $1 / \beta$ correspond to down-going events. 


\begin{tabular}{|c|c|c|c|c|}
\hline PCUP & Data & \multicolumn{3}{|c|}{ MC Expectation } \\
C11ts & & cosmic muons & $\nu_{\mu} / \bar{\nu}_{\mu}$ CC & $\nu_{e} / \bar{\nu}_{e}$ CC, NC \\
\hline Track Q11ality & 1172 & $840 \pm 30$ & $24.1 \pm 4.8$ & $0.3 \pm 0.1$ \\
Track Length & 344 & $275 \pm 17$ & $20.7 \pm 4.1$ & $0.1 \pm 0.0$ \\
Topology & 275 & $240 \pm 16$ & $19.4 \pm 3.9$ & $0.1 \pm 0.0$ \\
Timing & 10 & $<1.3$ & $15.1 \pm 3.0$ & $<0.1$ \\
\hline Total & 10 & \multicolumn{3}{|c|}{$15.1 \pm 3.0$} \\
\hline
\end{tabular}

Table 8.4: The number of events selected at each stage of the PCUP event selection. The expected signal is calculated for no oscillations. The errors quoted for $M C$ cosmic muons are calculated by adding the statistical error in quadrature with a $10 \%$ systematic error in the normalization; the errors quoted for the $M C$ atmospheric neutrinos are given by a $20 \%$ systematic error in the normalization.

\subsubsection{Results}

The numbers of events selected after each stage of the PCLP selection are shown in Table 8.4. The errors are calculated in the same way as for the FC/PCDN selection. The data and $\mathrm{MC}$ are found to be in reasonable agreement throughout the event selection, although there is an excess of data in the carly stages. A total of 10 events are selected from the data. This compares with a MC expectation of $15.1 \pm 3.0$ atmospheric $\nu_{\mu} / \bar{\nu}_{\mu}$ $\mathrm{CC}$ events, and $<0.1$ atmospheric $\nu_{e} / \bar{\nu}_{e} \mathrm{CC}$ and $\mathrm{NC}$ events. The $\mathrm{MC}$ expectation for cosmic muons is zero, with an upper limit of 1.3 events at $68 \%$ confidence.

The error on the MC cosmic muon background represents a significant fraction of the MC atmospheric neutrino signal. This is because the size of the MC cosmic muon sample is similar to the data set. A MC cosmic muon sample corresponding to a larger detector exposure was used to reduce the error. It was found that more than $95 \%$ of the cosmic muons that satisfy the topology cuts are in the encrgy range $0-2 \mathrm{GcV}$. Therefore, a sample of $2 \mathrm{M}$ cosmic muons was generated in this energy range, corresponding to a detector exposure of 4.76 Yrs. The number of events selected from this sample is zero, with an upper limit of 0.2 events at $68 \%$ confidence. 


\begin{tabular}{|c|c|}
\hline & $\left\langle E_{\nu}\right\rangle / \mathrm{GeV}$ \\
\hline FC & 1.9 \\
PCDN & 6.3 \\
PCLP & 7.5 \\
\hline All Events & 3.4 \\
\hline
\end{tabular}

Table 8.5: The mean energy of selected atmospheric $\nu_{\mu} / \bar{\nu}_{\mu}$ CC events.

\section{7 $\quad$ Selection Efficiencies}

The efficiency for selecting atmospheric $\nu_{\mu} / \bar{\nu}_{\mu} \mathrm{CC}$ events varies with encrgy and direction and is also different for each type of event. The selection efficiencies are determined as a function of energy and angle using a sample of MC atmospheric $\nu_{\mu} / \bar{\nu}_{\mu} \mathrm{CC}$ cvents which interact inside the fiducial volume and produce a muon which crosses $\geq 6$ planes. The selection efficiency is defined as the percentage of events that pass the selection cuts.

Figure 8.24 shows the selection efficiencies and signal distributions as a function of true neutrino energy for each type of event. The requirements placed on the length of muon tracks, in particular the additional constraints placed on PC events, remove the majority of neutrinos with sub-GeV energies. The effective neutrino energy threshold is approximately $600 \mathrm{MeV}$. Table 8.5 lists the mean energy of selected neutrino events for each type of event. The mean energy of FC events is lower than PC cvents since the $\mathrm{FC}$ events are required to begin and end inside the fiducial volume of the detector. The overall mean energy is $3.4 \mathrm{GeV}$. Figure 8.25 shows the selection efficiencies and signal distributions as a function of true muon zenith angle for each type of event. The detector geometry and containment cuts used to select FC/PCDN events preferentially remove neutrinos incident at small angles to the detector planes. Therefore the angular distribution of selected events has a broad peak around zero.

The selection efficiency is calculated to be: $57.1 \%$ for FC events; $44.2 \%$ for PCDN events; and $43.7 \%$ for PCUP events. The efficiency for PC events is lower than FC events since due to the additional track length requirements placed on the PC events. The overall selection efficiency is $52.3 \%$. 

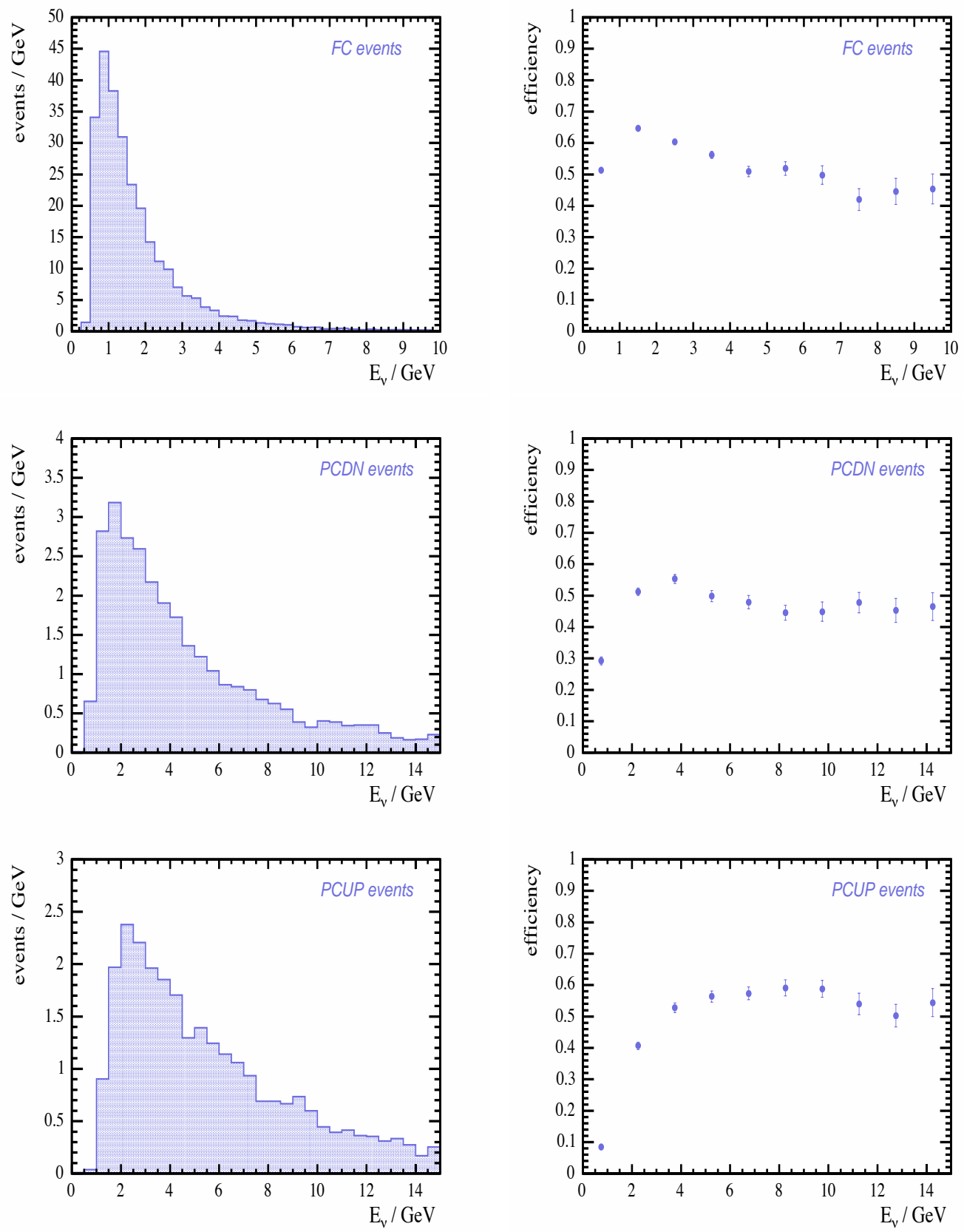

Figure 8.24: Distribution of selected events and event selection efficiencies as a function of neutrino energy for $M C$ atmospheric $\nu_{\mu} / \bar{\nu}_{\mu} C C$ events. 

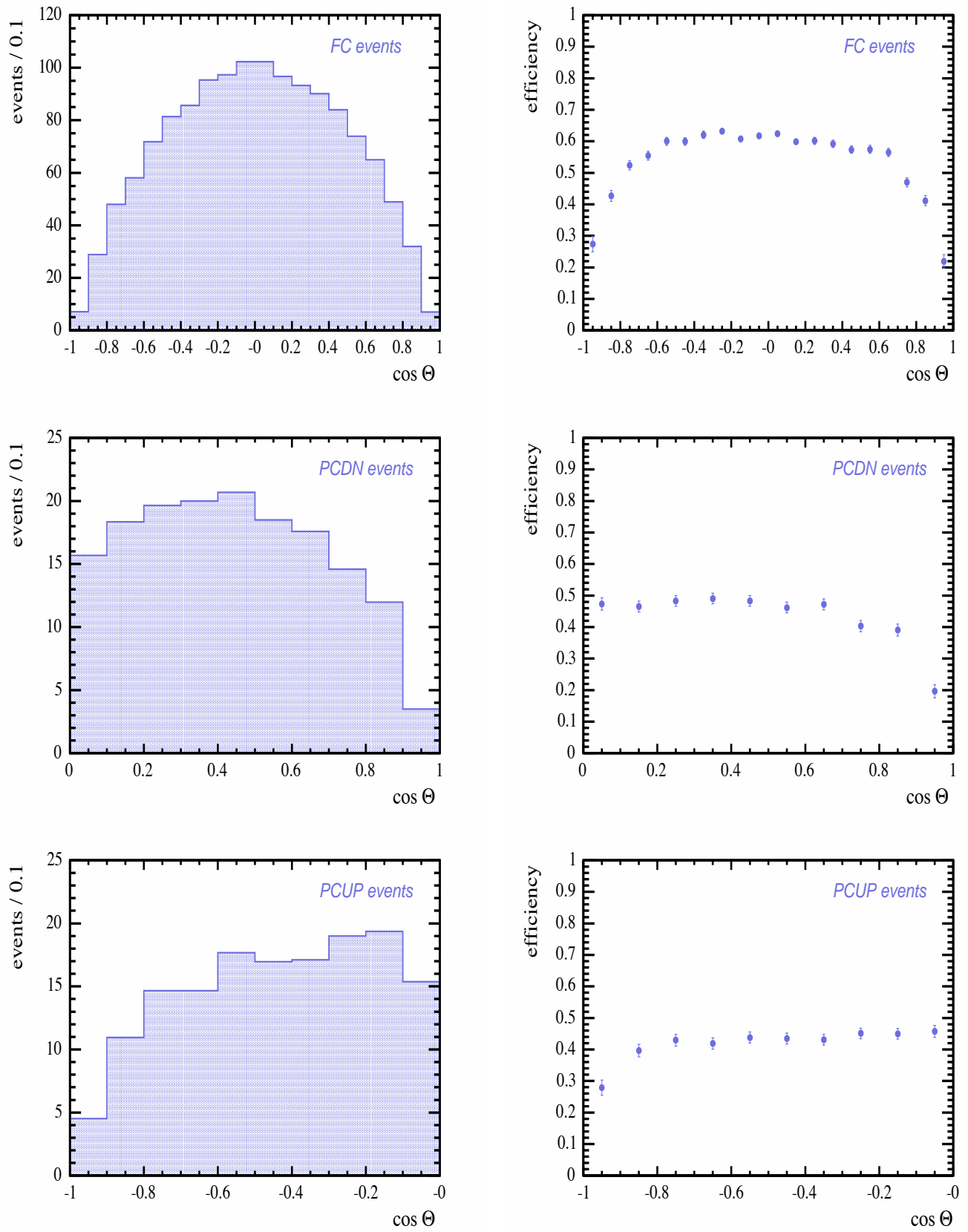

Figure 8.25: Distributions of selected events and event selection efficiencies as a function of muon zenith angle for $M C$ atmospheric $\nu_{\mu} / \bar{\nu}_{\mu} C C$ events. 


\subsection{Other Backgrounds}

\subsubsection{Neutrons}

Cosmic muons interact in the rock above the detector to produce showers of hadrons. A small number of these showers can enter the detector unaccompanied by the muon and produce events that appear contained in the detector. In particular, neutrons can interact inside the fiducial volume of the detector to produce contained proton tracks that are mistaken for neutrino interactions.

In order to measure this background, cosmic muon interactions were generated in the rock using the Geant4 simulation [147] and neutrons with a kinctic energy of $>300$ $\mathrm{MeV}$ were selected [144]. These neutrons were found to occur at a rate of approximately 40 events/day. A neutron sample corresponding to a detector exposure of 2.79 Yrs was analysed. The majority of neutrons interact inside the detector to produce small hadronic showers or short proton tracks. Only $10 \%$ of events pass the digit filters and $65 \%$ of these events do not contain a reconstructed track. For events that do contain a track, $99 \%$ begin outside the fiducial volume or do not pass the track selection cuts. The remaining events are removed in the early stages of the FC/PCDN and PCLP event selections. Therefore the neutron background is believed to be negligible.

\subsubsection{Neutrino-Induced Up-Going Muons}

Neutrinos interact in the rock below the detector to produce muons that are incident on the detector at a rate of approximately 0.65 cvents/day. Neutrino-induced muons incident at small angles to the planes can enter the scintillator inside the fiducial volume of the detector and be mistaken for contained neutrino interactions.

In order to determine this background, neutrino interactions were generated below the detector as described in [118]. A muon sample corresponding to a detector exposure of 200 Yrs was analysed. The majority of neurino-induced muons are through-going and so $65 \%$ of events do not pass the digit filters. For events that contain a reconstructed track, $97 \%$ begin outside the fiducial volume or do not pass the track selection cuts. The FC/PCDN and PCUP event selections each remove $70 \%$ of the remaining events. The up-going muon background in the data is estimated to be $0.5 \pm 0.1$ events. 


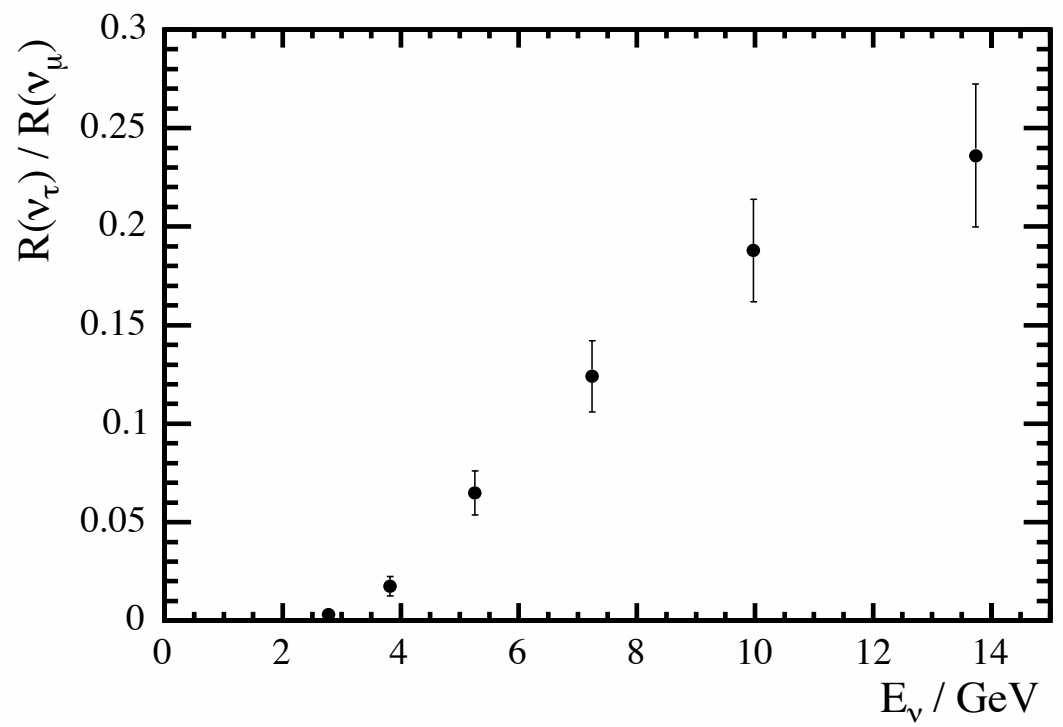

Figure 8.26: Ratio between the rates of selected $M C$ atmospheric $\nu_{\tau} / \bar{\nu}_{\tau} C C$ and $\nu_{\mu} / \bar{\nu}_{\mu}$ CC events as a function of neutrino energy.

\subsubsection{Tau Neutrinos}

If $\nu_{\mu} \leftrightarrow \nu_{\tau}$ oscillations occur in atmospheric neutrinos, an additional background will be generated by $\nu_{\tau} / \bar{\nu}_{\tau} \mathrm{CC}$ interactions where the tau lepton decays into a muon. The oscillation analysis must take account of the oscillated $\nu_{\tau} / \bar{\nu}_{\tau}$ CC events that are selected as $\nu_{\mu} / \bar{\nu}_{\mu} \mathrm{CC}$ events.

In order to determine this background, atmospheric $\nu_{\tau} / \bar{\nu}_{\tau}$ events were generated with the same energy spectrum as atmospheric $\nu_{\mu} / \bar{\nu}_{\mu}$ events. A neutrino sample corresponding to a detector exposure of $370 \mathrm{kT}$-Yrs was analysed. The rate of $\nu_{\tau} / \bar{\nu}_{\tau} \mathrm{CC}$ interactions is suppressed since the neutrino energy must be above the $3 \mathrm{GeV}$ threshold required to produce a tau lepton. The fraction of events containing muon tracks is also limited by the $17.4 \%$ branching ratio [105] for the decay of tau leptons into muons. Figure 8.26 shows the ratio between the rate of selected $\nu_{\tau} / \bar{\nu}_{\tau}$ CC events and the rate of selected $\nu_{\mu} / \bar{\nu}_{\mu} \mathrm{CC}$ events as a function of neutrino energy. This has a threshold at approximately $3 \mathrm{GeV}$ and rises above $20 \%$ at $10 \mathrm{GeV}$. The overall $\nu_{\tau} / \bar{\nu}_{\tau} \mathrm{CC}$ event rate is $3 \%$ of the $\nu_{\mu} / \bar{\nu}_{\mu}$ CC event rate. For the case of full $\nu_{\mu} \rightarrow \nu_{\tau}$ oscillations, the MC expectation for a detector exposure equivalent to the data is $3.3 \pm 0.6$ events. 


\subsection{Systematic Uncertainties in Event Selection}

The MC expectation is dependent on the physics models and detector conditions used in the simulation of atmospheric neutrinos. In order to determine the systematic uncertainties in the event selection, additional $\mathrm{MC}$ atmospheric neutrino samples have been generated using alternative physics models and detector conditions (sec Section 6.5). For each alternative $\mathrm{MC}$ sample, the difference in the MC expectation relative to the central MC sample is taken as the systematic crror in the MC expectation. Table 8.6 lists the systematic errors calculated in the MC expectation. The overall systematic error is estimated to be $20 \%$ for the atmospheric neutrino signal and $10 \%$ for the cosmic muon background. The systematic effects are discussed in detail below:

- Flux Model : The uncertaintics in the atmospheric ncutrino flux model give rise to large systematic errors in the $\mathrm{MC}$ expectation. An uncertainty of $15 \%$ is assigned to the absolute atmospheric neutrino flux (see Section 6.4.1). In addition, uncertaintics of $5 \%$ are assigned to the Up/Down and $\bar{\nu}_{\mu} / \nu_{\mu}$ flux ratios while keeping the overall flux constant. This produces systematic errors of $1 \%$ and $2 \%$ respectively in the MC expectation.

- Cross-Section Model : The uncertainties in the neutrino cross-section model also give rise to large systematic errors in the $\mathrm{MC}$ expectation. An uncertainty of $10 \%$ is assigned to the overall neutrino cross-section (see Section 6.4.2). In addition, the relative cross-section for quasi-clastic neutrino interactions is varied by $\pm 20 \%$ [148] while keeping the overall cross-section constant. This produces a systematic error of $4 \%$ in the MC expectation.

- Hadronic Model : The reconstruction and selection of neutrino events depends to a small degrec on the model used to propagate hadronic particles produced in neutrino interactions. In order to estimate the systematic error due to the choice of hadronic model, the GCALOR model [141] used for the central MC sample is replaced by the $G H E I S H A$ model [143], which has also becn found to give a good description of the detector response to single hadrons. This produces a systematic crror of $3 \%$ in the MC expectation.

- Timing Resolution : Timing information is used at a number of stages of the reconstruction and selection of events. In order to determine the effect of 


\begin{tabular}{|c|c|}
\hline & $\begin{array}{c}\text { Systematic Error } \\
\text { in Event Sclection }\end{array}$ \\
\hline Neutrino Flux & $15 \%$ \\
Up/Down Ratio $( \pm 5 \%)$ & $1 \%$ \\
$\bar{\nu}_{\mu} / \nu_{\mu}$ Ratio $( \pm 5 \%)$ & $2 \%$ \\
Neutrino Cross-Section & $10 \%$ \\
QE Cross-Section $( \pm 20 \%)$ & $4 \%$ \\
Hadronic Model $($ Gheisha $)$ & $3 \%$ \\
Timing Resolution $( \pm 0.1 n s)$ & $1 \%$ \\
Energy Calibration $( \pm 5 \%)$ & $1 \%$ \\
Cosmic Muon Normalization $( \pm 10 \%)$ & $<1 \%$ \\
\hline
\end{tabular}

Table 8.6: Systematic errors in MC expectation, calculated using $M C$ samples generated with alternative physics models and detector conditions.

the timing resolution on the event selection, additional MC samples have becn generated with the timing smearing adjusted to vary the resolution by \pm 0.1 ns. This produces a systematic error of $1 \%$ in the MC expectation.

- Energy Calibration : Pulse height information is used at a number of stages of the reconstruction and selection of events. The error in the pulse height calibration is estimated to be 5\% [89]. In order to estimate the effect of the pulse height calibration on the event selection, additional MC samples have been generated with the overall light output adjusted by $\pm 5 \%$. This produces a systematic crror of $1 \%$ in the $\mathrm{MC}$ expectation.

- Cosmic Muon Normalization : The normalization of the MC cosmic muon sample is determined by comparing the number of events selected in data and MC, but the relative normalization of data and $\mathrm{MC}$ varies with energy and direction. In order to account for this, an uncertainty of $10 \%$ is assigned to the normalization. The expected cosmic muon background is determined using the veto shicld with an uncertainty of approximately $10 \%$. This produces a systematic error of $<1 \%$ in the overall MC expectation. 


\begin{tabular}{|c|c|c|c|c|c|}
\hline & Data & \multicolumn{4}{|c|}{ MC Expectation (no oscillations) } \\
& & cosmic muons & $\nu_{\mu} / \bar{\nu}_{\mu} \mathrm{CC}$ & $\nu_{e} / \bar{\nu}_{e} \mathrm{CC}, \mathrm{NC}$ & $\nu$-induced $\mu$ \\
\hline FC & 56 & $2.8 \pm 0.4$ & $70.7 \pm 14.1$ & $3.8 \pm 0.8$ & $0.3 \pm 0.1$ \\
PCDN & 16 & $0.7 \pm 0.3$ & $16.2 \pm 3.2$ & $<0.1$ & $0.1 \pm 0.0$ \\
PCUP & 10 & $<0.2$ & $15.1 \pm 3.0$ & $<0.1$ & $0.1 \pm 0.0$ \\
\hline Total & 82 & $3.5 \pm 0.5$ & $102.0 \pm 20.4$ & $3.9 \pm 0.8$ & $0.5 \pm 0.1$ \\
\hline
\end{tabular}

Table 8.7: Summary of expected/observed events for each type of event. The cosmic muon background is calculated by applying the measured shield efficiencies to the vetoed events. Neutrino-induced muons are produced by atmospheric $\nu_{\mu} / \bar{\nu}_{\mu} C C$ interactions in the rock below the detector.

\subsection{Selected Events}

The results of the event selection are summarized in Table 8.7. A total of 82 candidate atmospheric $\nu_{\mu} / \bar{\nu}_{\mu}$ CC events are selected from the data. This compares with a MC expectation of $109.9 \pm 21.4$ events consisting of: $3.5 \pm 0.5$ cosmic muons; $102.0 \pm 20.4$ atmospheric $\nu_{\mu} / \bar{\nu}_{\mu}$ CC events; $3.9 \pm 0.8$ atmospheric $\nu_{e} / \bar{\nu}_{e} \mathrm{CC}$ and atmospheric NC cvents; and $0.5 \pm 0.1$ neutrino-induced up-going muons (in the absence of oscillations). A full list of the candidate atmospheric $\nu_{\mu} / \bar{\nu}_{\mu}$ CC events is included in Appendix A. Figures 8.27-8.29 show some examples of the selected events.

The atmospheric neutrino interactions are expected to be uniform in space and time. The distribution of interaction points reconstructed from the selected events is shown for the X-Y plane in Figure 8.30, and along the Z-axis in Figure 8.31. The selected events appear evenly spread across the detector. Figure 8.32 shows the measured rate of events for successive intervals of three weeks. This also appears uniform, with the overall event rate measured to be 0.26 events/day. This compares with a MC expectation of $0.35 \pm 0.07$ events/day. The observed number of events is consistent with the MC expectation to approximately one standard deviation. 

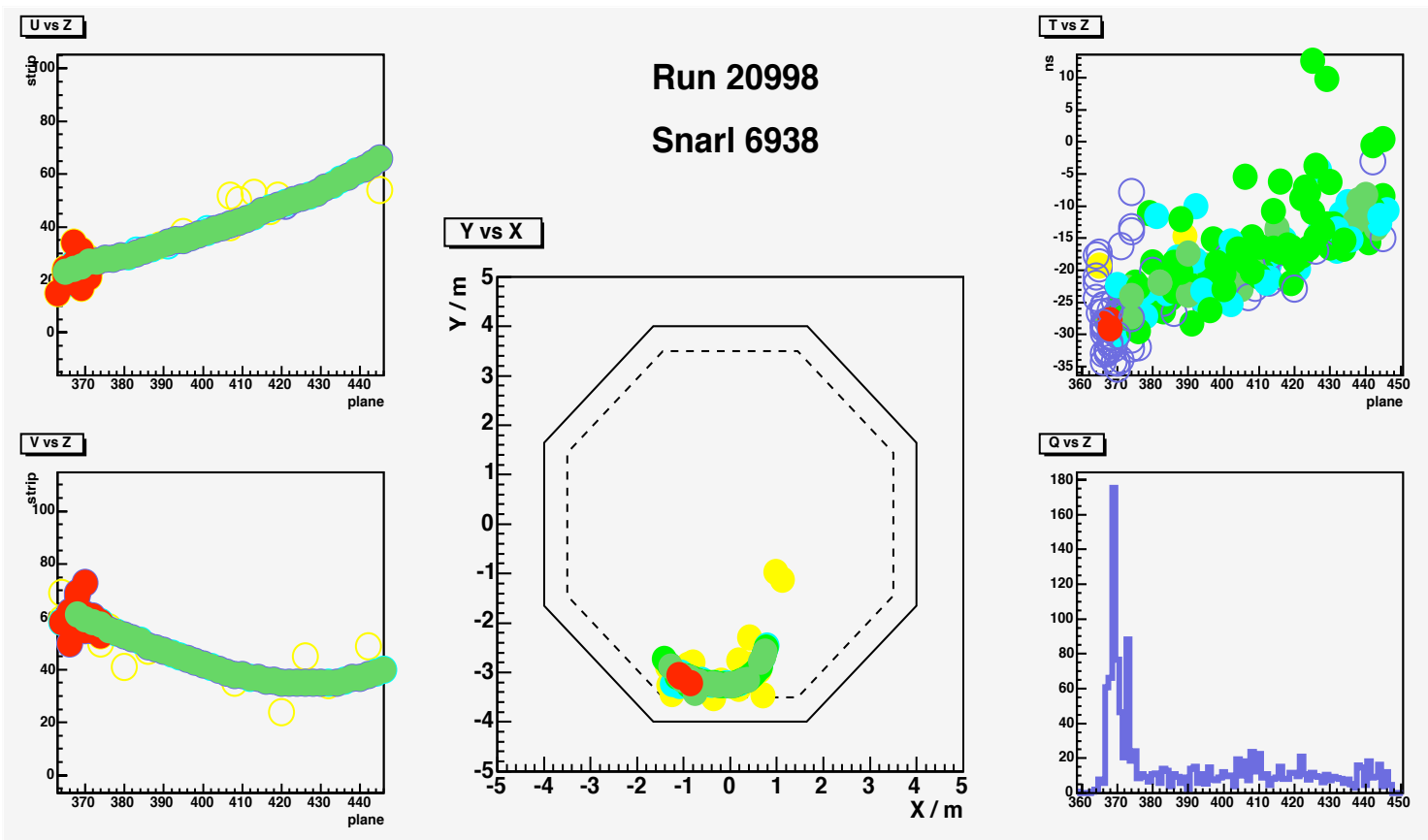

Figure 8.27: Example of FC atmospheric $\nu_{\mu} \bar{\nu}_{\mu} C C$ event. The panels on the left show the hits in the $U-Z$ and $V$-Z views, with the green points representing the reconstructed track and the red points representing reconstructed vertex shower. The central panel shows the $X$-Y view. The panel on the top right shows the measured times of hits in the event as a function of plane number. The panel on the bottom right shows the pulse height deposited in each plane of the event as a function of the plane number. 

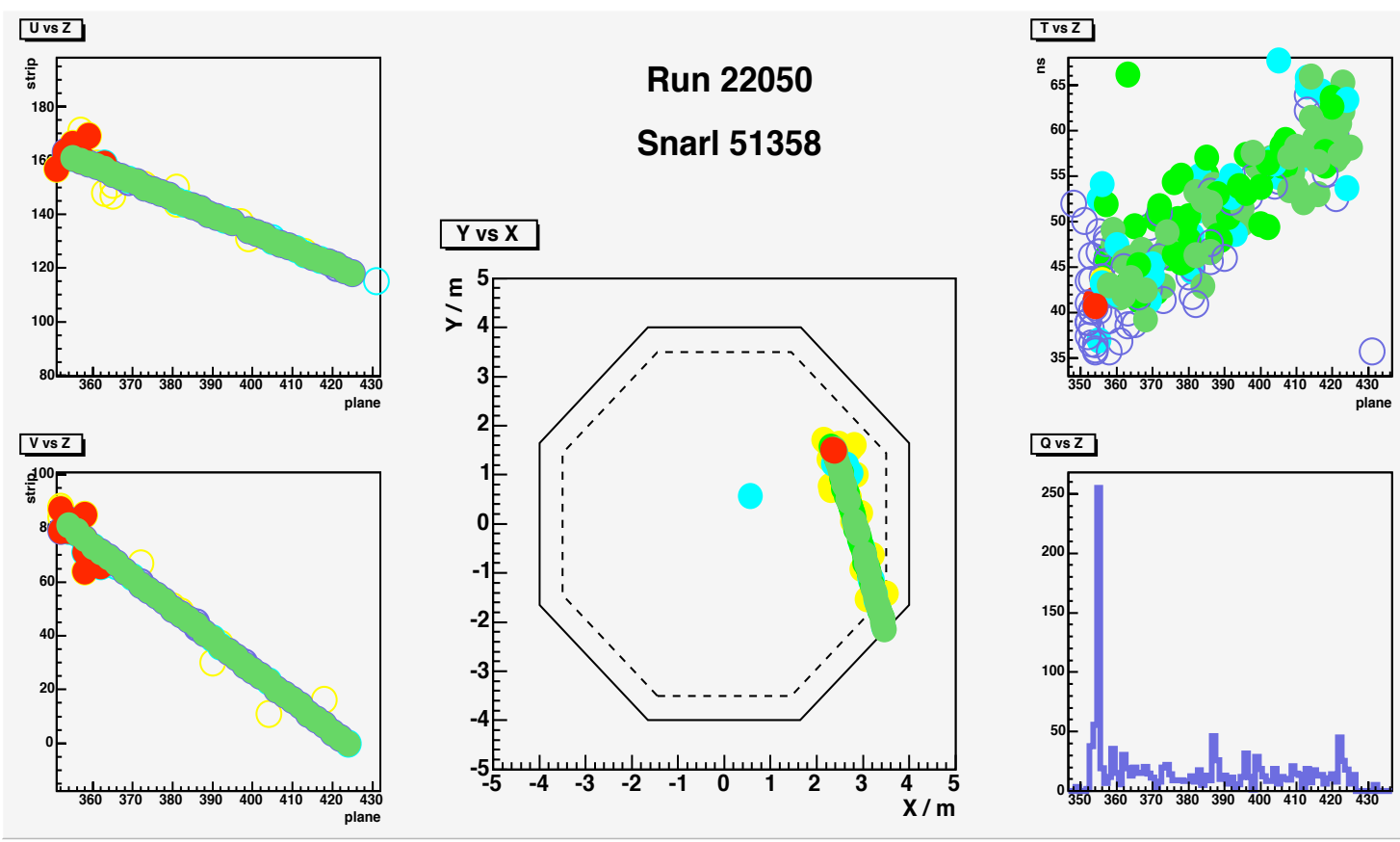

Figure 8.28: Example of PCDN atmospheric $\nu_{\mu} / \bar{\nu}_{\mu} C C$ event.
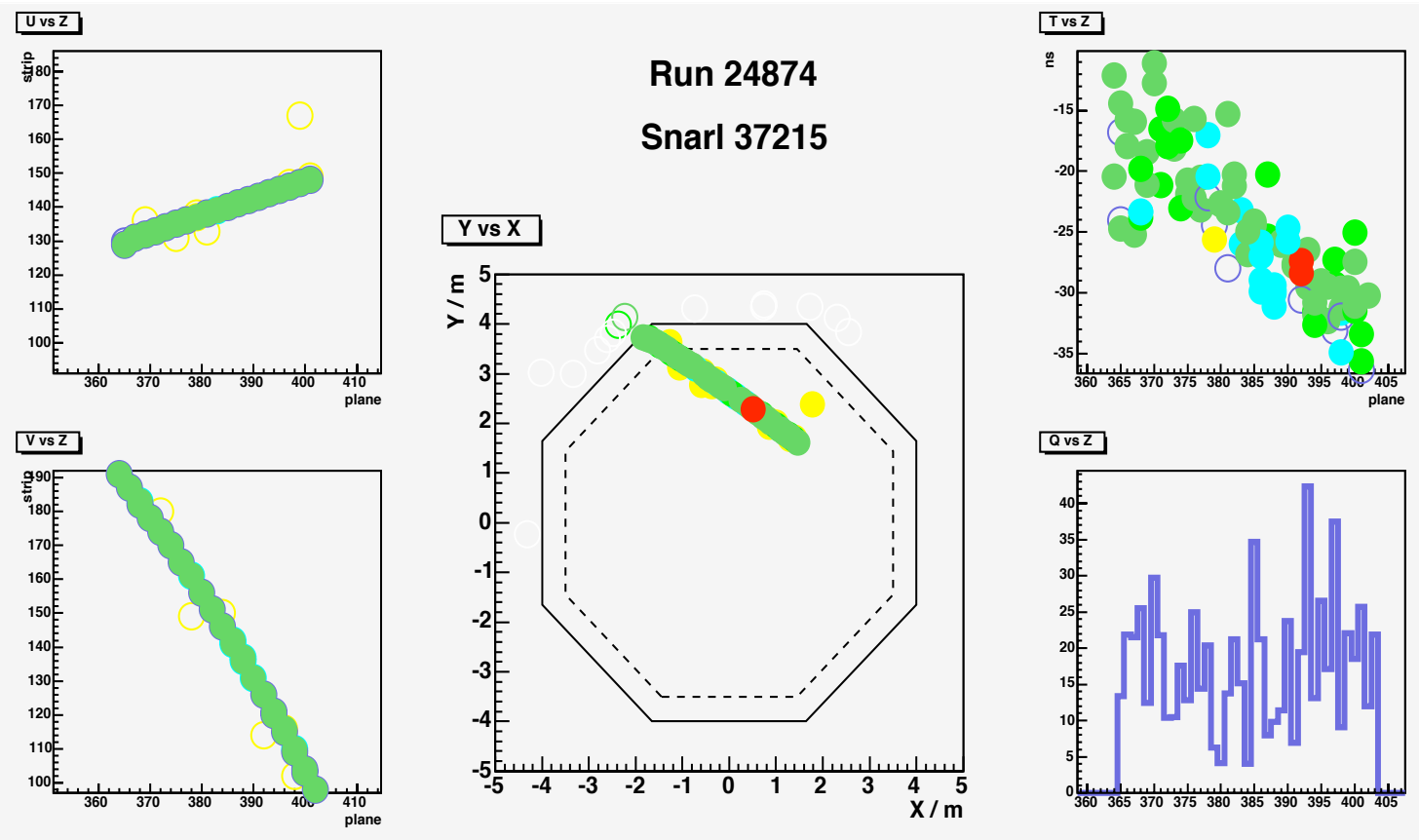

Figure 8.29: Example of PCUP atmospheric $\nu_{\mu} / \bar{\nu}_{\mu} C C$ event. 


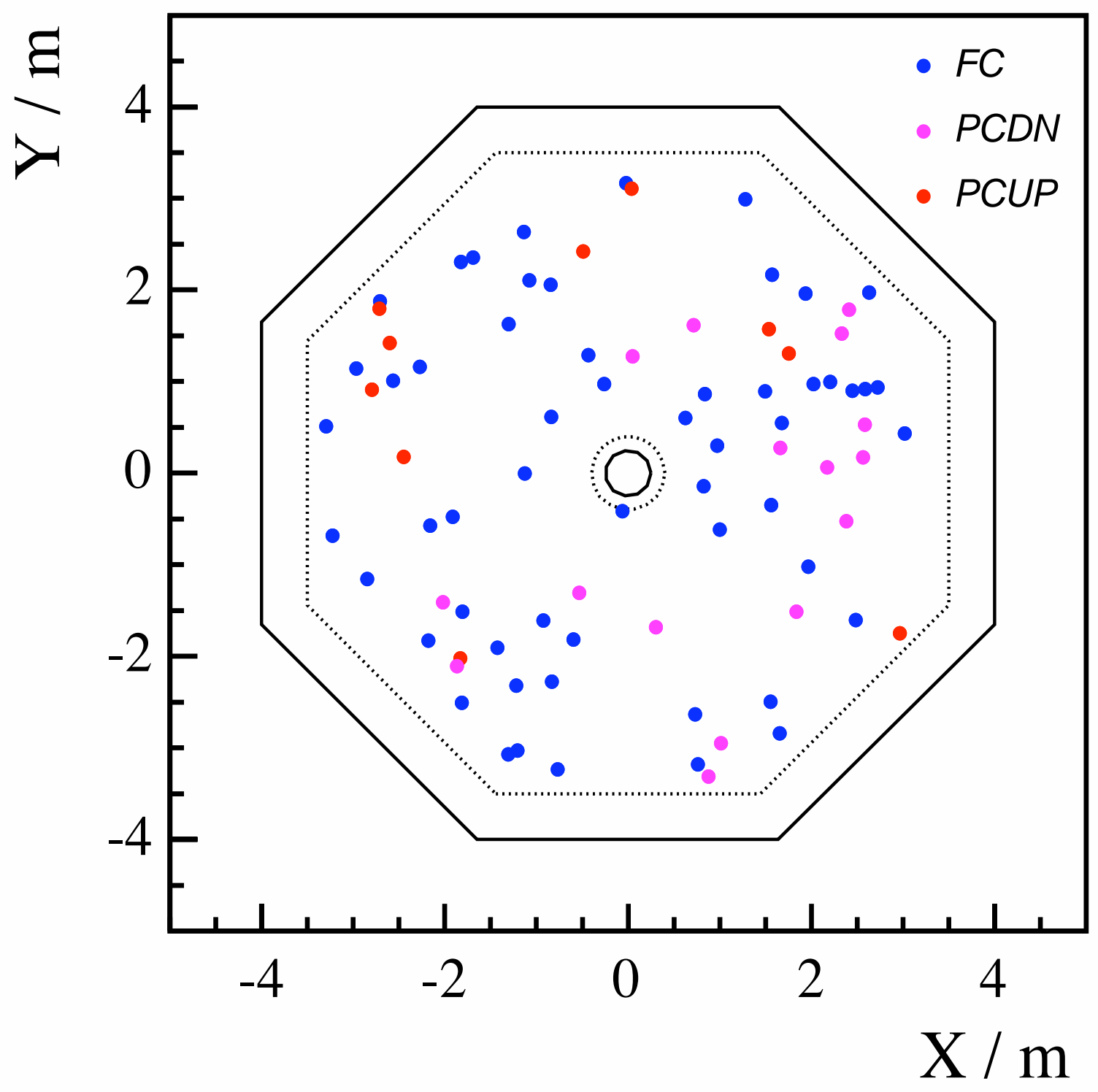

Figure 8.30: Distribution of interaction points in the $x$-y plane for selected atmospheric $\nu_{\mu} / \bar{\nu}_{\mu} C C$ events. The $F C$ events are indicated by blue circles, PCDN events by magenta circles, and PCUP events by red circles. The solid lines represent the edges of the detector, and the dotted lines indicate the boundaries of the fiducial volume. 


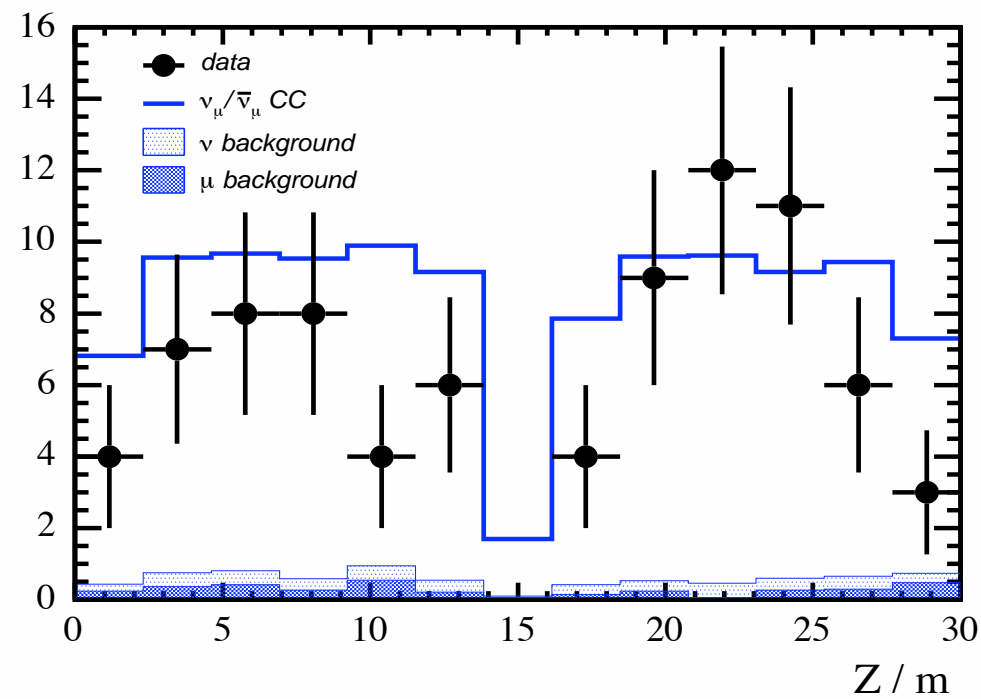

Figure 8.31: Distribution of interaction points along the z-axis for selected atmospheric $\nu_{\mu} / \bar{\nu}_{\mu} C C$ events. The region less than $15 \mathrm{~m}$ represents supermodule 1 and the region greater than $15 \mathrm{~m}$ represents supermodule 2. The distribution of cosmic muon background is obtained by scaling the distribution of vetoed events using the measured shield efficiencies.

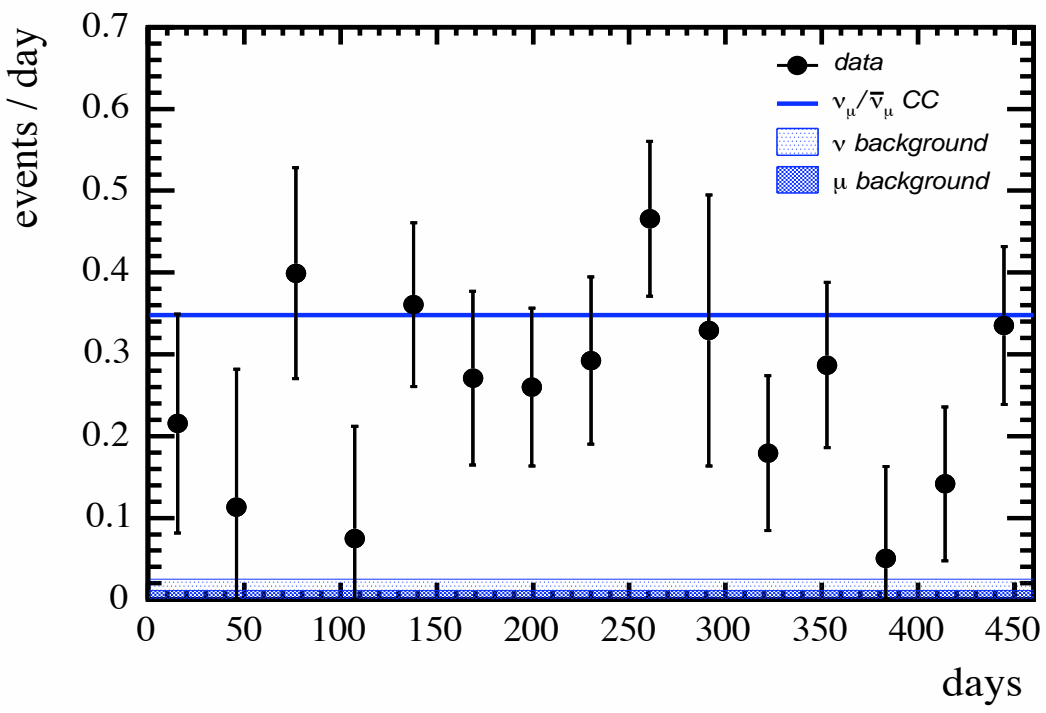

Figure 8.32: Distribution of neutrino event rate over time. The data is divided into 3-week intervals and the event rate is calculated for each interval. The number of days is defined relative to August 1st 2003. 


\section{Chapter 9}

\section{Neutrino Oscillation Analysis}

\subsection{Analysis Overview}

The measured and expected distributions of selected events are compared in order to test the consistency of the data with $\nu_{\mu k} \leftrightarrow \nu_{\tau}$ oscillations and measure the oscillation parameters $\Delta m_{23}^{2}$ and $\sin ^{2} 2 \theta_{23}$. The signature of neutrino oscillations is a deficit in the observed atmospheric $\nu_{\mu} / \bar{\nu}_{\mu} \mathrm{CC}$ events relative to the expectation at high values of $L / E$. Since the selected data set is small, the oscillation parameters cannot be determined with high precision. Table 9.1 lists the expected number of events for no oscillations, and for oscillations with $\Delta m_{23}^{2}=2.5 \times 10^{-3} \mathrm{eV}^{2}, \sin ^{2} 2 \theta_{23}=1.0$. The data is consistent with both scenarios to within one standard deviation. The selected data set can be used to determine the atmospheric neutrino up/down ratio and $\bar{\nu}_{\mu} / \nu_{\mu}$ ratio with reasonable precision. These ratios provide measures of the compatibility of the data with the simulation and with the oscillation hypothesis.

In order to measure the $u p /$ down and $\bar{\nu}_{\mu} / \nu_{\mu}$ ratios and to perform an oscillation fit, a high resolution sample of events is selected with well-measured direction and charge. Events with a well-measured direction are used to determine the up/down ratio; events with a well-measured charge are used to determine the $\bar{\nu}_{\mu} / \nu_{\mu}$ ratio. A maximum likelihood fit is applied to the reconstructed $L / E$ distribution to measure the oscillation parameters. An oscillation fit is first performed on the combined sample of neutrino and anti-neutrinos. The events are then divided into neutrino and anti-neutrinos and a second oscillation fit is performed. Finally, the projected sensitivity to oscillations is determined for a detector exposure of $25 \mathrm{kT}$-Yrs (approximately 5 years running). 


\begin{tabular}{|c|c|c|c|c|c|c|}
\hline & Data & Cosmic muons & $\nu_{\mu} / \bar{\nu}_{\mu} \mathrm{CC}$ & $\nu_{\tau} / \bar{\nu}_{\tau} \mathrm{CC}$ & $\nu_{e} / \bar{\nu}_{e} \mathrm{CC}, \mathrm{NC}$ & $\nu$-induced $\mu$ \\
\hline FC+PC & & \multicolumn{5}{|c|}{ Expectation ( no oscillations $)$} \\
cvents & 82 & $3.5 \pm 0.3$ & $102.0 \pm 20.4$ & $<0.1$ & $3.9 \pm 0.8$ & $0.5 \pm 0.1$ \\
\hline $\begin{array}{c}\text { FC+PC } \\
\text { events }\end{array}$ & 82 & $3.5 \pm 0.3$ & $73.0 \pm 14.6$ & $0.9 \pm 0.2$ & $3.9 \pm 0.8$ & $0.4 \pm 0.1$ \\
\hline
\end{tabular}

Table 9.1: Comparison between expected and observed numbers of events for no oscillations, and for oscillations with $\Delta m_{23}^{2}=2.5 \times 10^{-3} \mathrm{eV}^{2}, \sin ^{2} 2 \theta_{23}=$ 1.0. The expected cosmic muon background is calculated from the data using $F C / P C D N$ events that have been tagged by the veto shield.

\subsection{Selection of High Resolution Events}

A high resolution sample of events is used to measure the propertics of the atmospheric neutrino flux: the up/down ratio is determined using events with a well-measured muon dircetion; the $\bar{\nu}_{\mu} / \nu_{\mu}$ ratio is determined using events with a well-measured muon charge. Events with a well-measured direction are selected by applying cuts on the muon length and on timing information; events with a well-measured charge are selected by applying additional cuts on the muon curvature. The following cuts are used to select high resolution events:

- Track Length : The efficiency for correctly reconstructing the direction and charge of atmospheric $\nu_{\mu} / \bar{\nu}_{\mu}$ events increases with the event length. Events which contain a larger number of hits or travel a longer distance have: a better timing resolution; a better discrimination between the start and end times of the muon track; a clearer muon track curvature; and a smaller background from $\nu_{e} / \bar{\nu}_{e} \mathrm{CC}$, NC events and from cosmic muons. In order to select the well-measured atmospheric $\nu_{\mu} / \bar{\nu}_{\mu}$ CC signal, muon tracks are required to cross $\geq 10$ planes and travel a distance of $>1 \mathrm{~m}$. Figure 9.1 shows the distribution of the number of muon track planes for data and MC. Figure 9.2 shows the distribution of the muon track range for data and $\mathrm{MC}$. The track length cuts remove $80 \%$ of signal events which have an incorrectly reconstructed direction. The cuts select $76.2 \%$ of signal events, with the event direction reconstructed correctly in $98.9 \%$ of selected events. 
- Muon Direction : The muon direction is reconstructed from timing information 11sing the method outlined in Section 7.4.1. Events with a well-measured direction are selected by analysing the up-going and down-going timing fits to muon tracks. A cut is applied on the difference between the rms deviations of cach timing fit, requiring the best fit to be significantly better than the alternative fit. Events are required to satisfy $\left|r m s_{u p}-r m s_{\text {down }}\right|>0.66$ ns. Figure 9.3 shows the distribution of $r m s_{u p}-r m s_{\text {doun }}$ for events which satisfy the track length cuts. The direction cut selects $93.6 \%$ of remaining signal events, with the muon direction reconstructed correctly in $99.6 \%$ of selected events.

- Muon Charge : The muon charge is reconstructed from the track curvature using the method outlined in Section 7.4.2. Events with a well-measured charge are selected by applying a cut on the calculated crror in $\langle Q / p\rangle$. Events with $\langle Q / p\rangle / \sigma_{\langle Q / p\rangle}<-2$ are classified as $\nu_{\mu}$; events with $\left.\langle Q / p\rangle / \sigma_{\langle Q / p\rangle}\right\rangle+2$ are classified as $\bar{\nu}_{\mu}$. Figure 9.4 shows the distribution of $\langle Q / p\rangle / \sigma_{\langle Q / p\rangle}$ for events which satisfy the track length and muon direction cuts. The muon charge cut selects $74.7 \%$ of the remaining signal events. Since the $\nu_{\mu}$ event rate is larger than the $\bar{\nu}_{\mu}$ event rate, the purity of the selected $\nu_{\mu}$ sample is higher than the purity of the selected $\bar{\nu}_{\mu}$ sample. For events classified as $\nu_{\mu}$, the muon charge is reconstructed correctly in $99.4 \%$ of cases; for events classified as $\bar{\nu}_{\mu}$, the muon charge is reconstructed correctly in $97.4 \%$ of cases.

The events satisfying the muon direction cuts are separated into up-going and downgoing events based on the reconstructed muon zenith angle. Table 9.2 lists the number of up-going and down-going events selected in data and MC. Of the 82 events selected from data, 60 events have a well-measured direction, with 22 events classified as up-going and 38 cvents classificd as down-going. This compares with an expectation of $37.4 \pm 7.4$ up-going events and $40.3 \pm 7.6$ down-going events. There is good agreement between data and MC for down-going events, but a deficit in the data for up-going events. Figure 9.5 shows the distribution of the reconstructed muon zenith angle for data and MC. The expected distributions are shown for no oscillations, and for oscillations with $\Delta m_{23}^{2}=2.5 \times 10^{-3} \mathrm{eV}^{2}, \sin ^{2} 2 \theta_{23}=1.0$. The data are more consistent with oscillations although the statistical errors are large. 


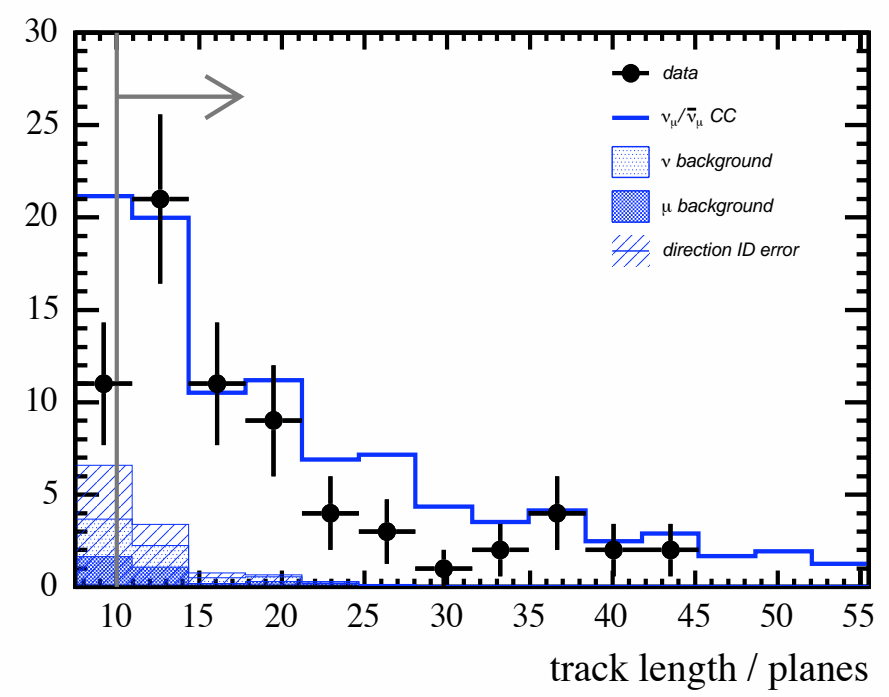

Figure 9.1: Distribution of muon track planes for selected $\nu_{\mu} / \bar{\nu}_{\mu}$ events. The data are represented by the points; the solid blue line represents the expectation for no oscillations. Events are required to contain $>10$ planes.

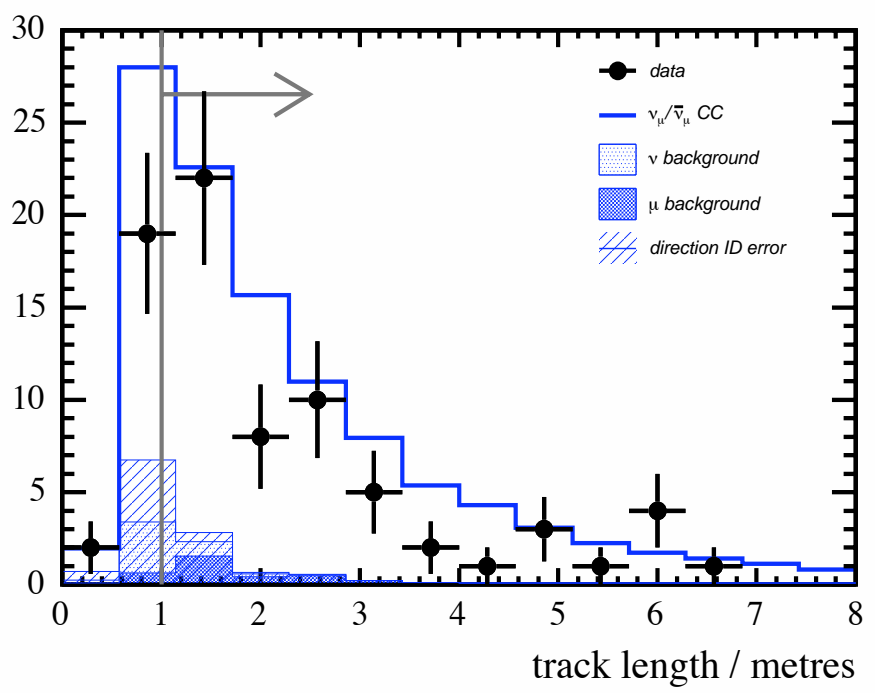

Figure 9.2: Distribution of muon range for selected $\nu_{\mu} / \bar{\nu}_{\mu}$ events. The data are represented by the points; the solid blue line represents the expectation for no oscillations. Events are required to be $>1.0 \mathrm{~m}$ in length. 


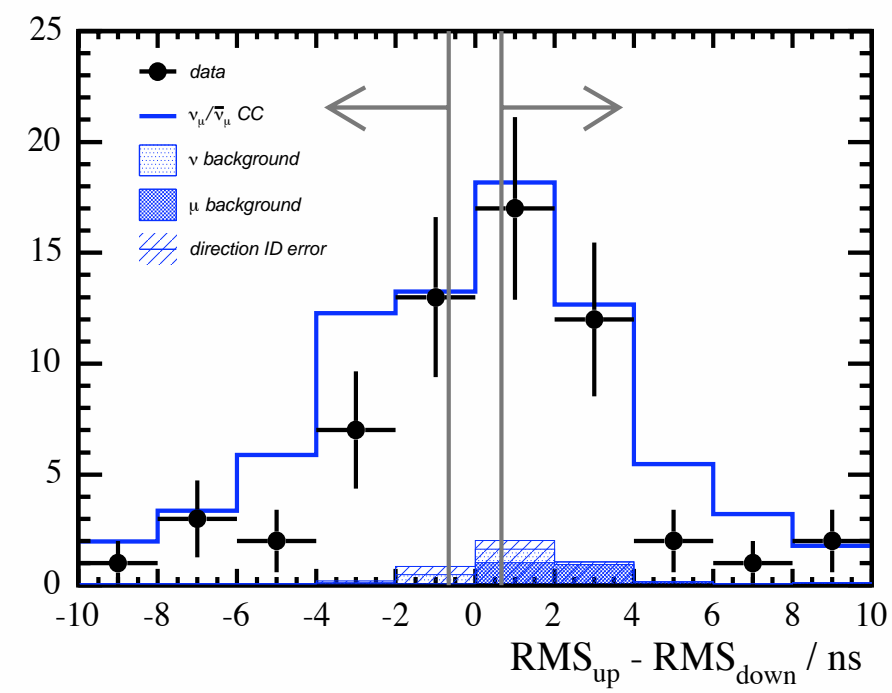

Figure 9.3: Distribution of $r m s_{u p}-r m s_{\text {down }}$ for events that satisfy the track length cuts. The data are represented by the points; the solid blue line represents the expectation for no oscillations. Events are required to satisfy $\mid$ rms $_{\text {up }}-r m s_{\text {down }} \mid>0.66$ ns.

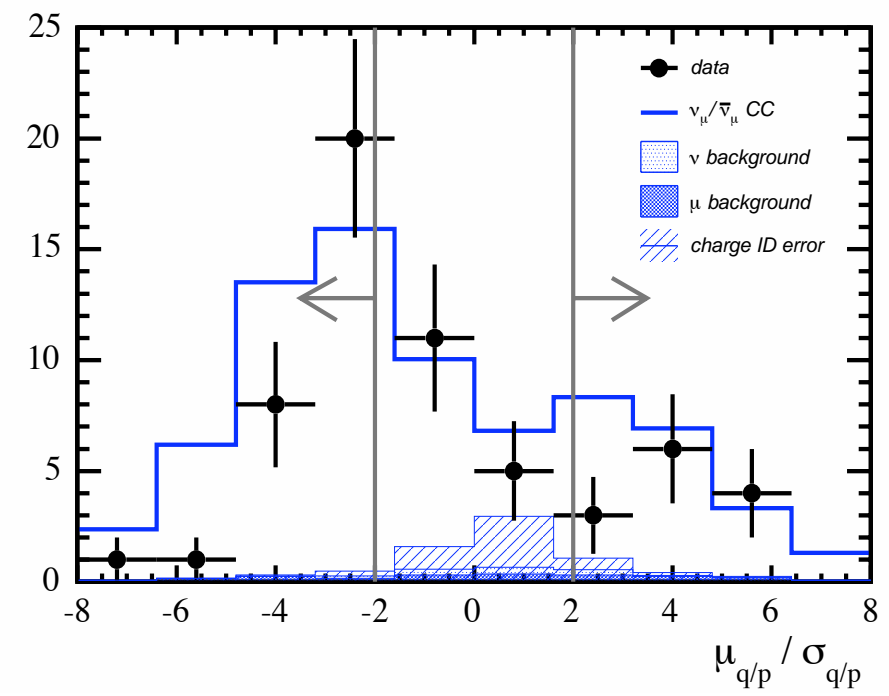

Figure 9.4: Distribution of $\langle Q / p\rangle / \sigma_{\langle Q / p\rangle}$ for atmospheric neutrino events that satisfy the track length and muon direction cuts. The data are represented by the points; the solid blue line represents the expectation for no oscillations. Events are required to satisfy $|\langle Q / p\rangle| / \sigma_{\langle Q / p\rangle}>2.0$. 


\begin{tabular}{|c|c|c|c|c|c|}
\hline & Data & \multicolumn{4}{|c|}{ Expectation ( no oscillations ) } \\
& & Cosmic muons & $\nu_{\mu} / \bar{\nu}_{\mu} \mathrm{CC}$ & $\nu_{e} / \bar{\nu}_{e} \mathrm{CC}, \mathrm{NC}$ & $\nu$-induced $\mu$ \\
\hline UP & 22 & $<0.1$ & $36.7 \pm 7.3$ & $0.4 \pm 0.1$ & $0.3 \pm 0.1$ \\
DOWN & 38 & $2.1 \pm 0.2$ & $37.7 \pm 7.5$ & $0 . \overline{0} \pm 0.1$ & $<0.1$ \\
$?$ & 22 & $1.4 \pm 0.1$ & $27.6 \pm 5.5$ & $3.0 \pm 0.6$ & $0.1 \pm 0.0$ \\
\hline
\end{tabular}

Table 9.2: Comparison between data and expectation for events selected as up-going and down-going, and events with ambiguous direction.

\begin{tabular}{|c|c|c|c|c|c|}
\hline & Data & \multicolumn{4}{|c|}{ Expectation ( no oscillations ) } \\
& & Cosmic muons & $\nu_{\mu} / \bar{\nu}_{\mu} \mathrm{CC}$ & $\nu_{e} / \bar{\nu}_{e} \mathrm{CC}, \mathrm{NC}$ & $\nu$-induced $\mu$ \\
\hline$\nu_{\mu}$ & 27 & $0.5 \pm 0.1$ & $35.1 \pm 7.0$ & $0.1 \pm 0.0$ & $0.1 \pm 0.0$ \\
$\bar{\nu}_{\mu}$ & 13 & $0.7 \pm 0.1$ & $18.1 \pm 3.6$ & $0.2 \pm 0.0$ & $0.1 \pm 0.0$ \\
$?$ & 42 & $2.3 \pm 0.2$ & $48.7 \pm 9.7$ & $3.6 \pm 0.7$ & $0.3 \pm 0.1$ \\
\hline
\end{tabular}

Table 9.3: Comparison betueen data and expectation for events selected as neutrinos and anti-neutrinos, and events with ambiguous charge.

\begin{tabular}{|c|c|c|c|c|c|c|}
\hline \multicolumn{2}{|c|}{} & Data & \multicolumn{4}{|c|}{ Expectation (no oscillations ) } \\
\hline \multirow{2}{|c|}{$\nu_{\mu}$} & UP & 11 & $<0.1$ & $17.4 \pm 3 . \overline{5}$ & $<0.1$ & $0.1 \pm 0.0$ \\
& DOWN & 16 & $0.5 \pm 0.1$ & $17.7 \pm 3.5$ & $<0.1$ & $<0.1$ \\
\hline $\bar{\nu}_{\mu}$ & UP & 5 & $<0.1$ & $9.0 \pm 1.8$ & $0.1 \pm 0.0$ & $0.1 \pm 0.0$ \\
& DOW. & 8 & $0.7 \pm 0.1$ & $9.1 \pm 1.8$ & $0.1 \pm 0.0$ & $<0.1$ \\
\hline
\end{tabular}

Table 9.4: Comparison between data and expectation for neutrinos and anti-neutrinos, separated into up-going and down-going events. 

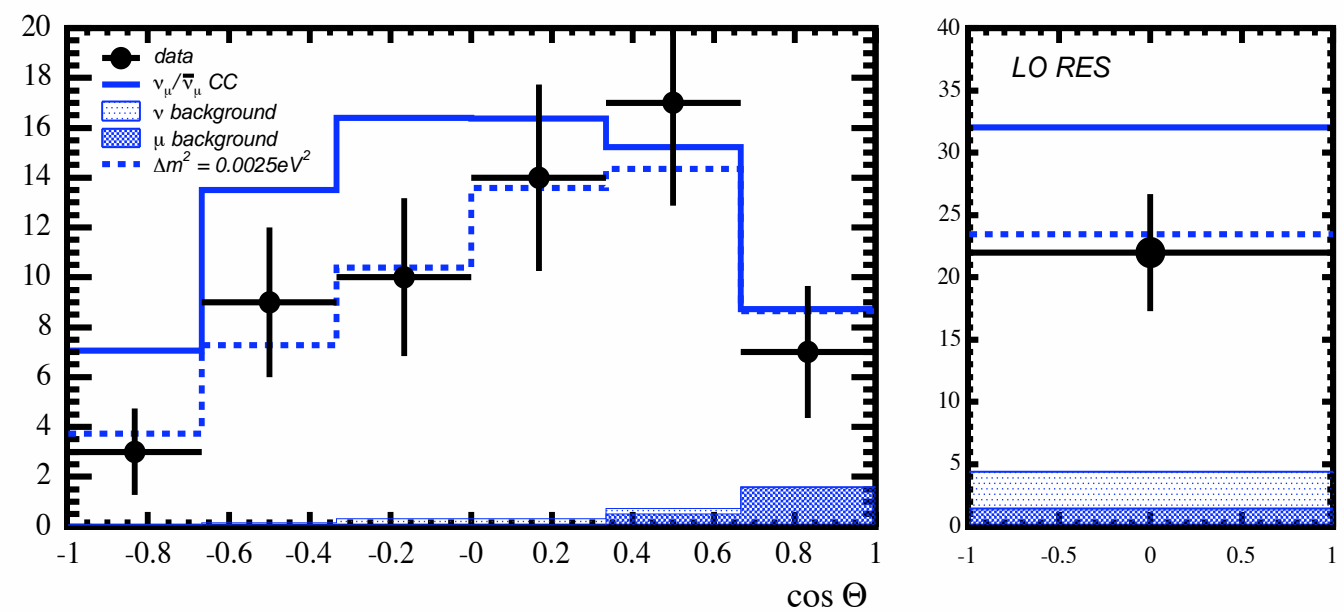

Figure 9.5: Distribution of reconstructed muon zenith angle for events with a well-measured direction (left) and events with ambiguous direction (right). The data are represented by the points; the solid blue line represents the expectation for no oscillations; the dashed blue line represents the expectation for oscillations with $\Delta m_{23}^{2}=2.5 \times 10^{-3} \mathrm{eV}^{2}, \sin ^{2} 2 \theta_{23}=1.0$.
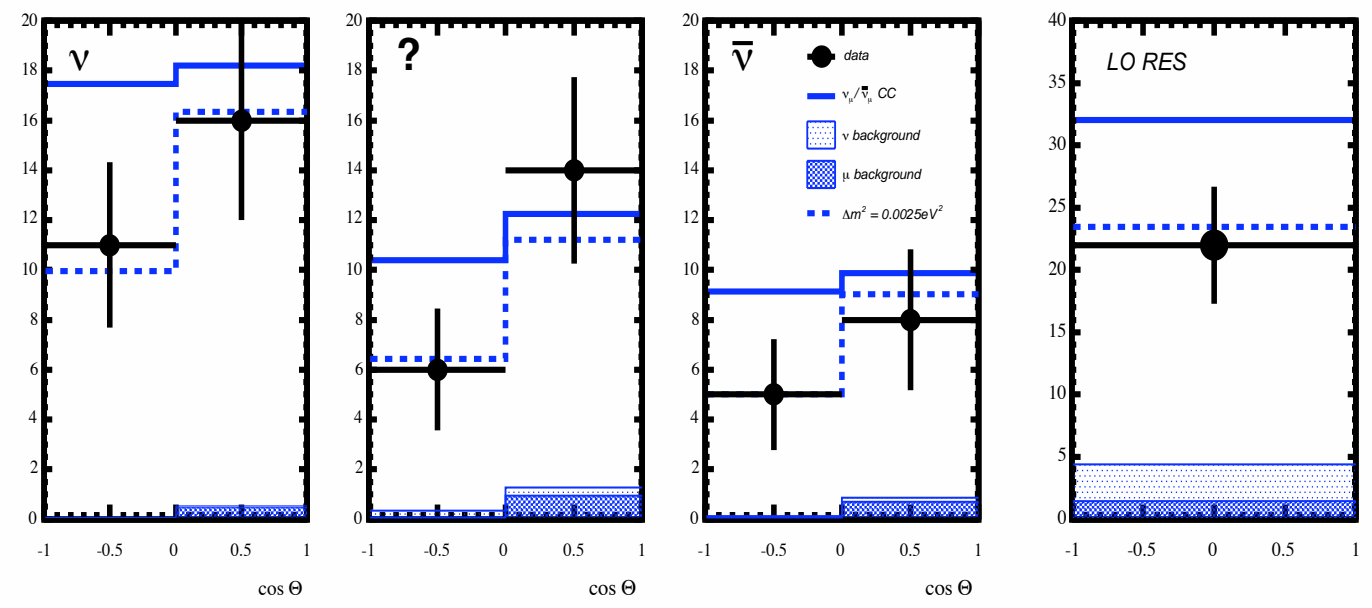

Figure 9.6: Distribution of reconstructed muon zenith angle for events classified as neutrinos $\left(\nu_{\mu}\right)$, events with an ambiguous charge (?), events classified as anti-neutrinos $\left(\bar{\nu}_{\mu}\right)$, and events with ambiguous direction (right). The data are represented by the points; the solid blue line represents the expectation for no oscillations; the dashed blue line represents the expectation for oscillations with $\Delta m_{23}^{2}=2.5 \times 10^{-3} \mathrm{eV}^{2}, \sin ^{2} 2 \theta_{23}=1.0$. 
The events satisfying the muon charge cuts are separated into neutrinos and antineutrinos based on the reconstructed muon charge. Table 9.3 lists the number of neutrinos and anti-neutrinos selected in data and MC. Of the 60 events with a well-measured direction, 40 events have a well-measured charge, with 27 cvents classified as neutrinos and 13 events classified as anti-neutrinos. This compares with an expectation of $35.8 \pm 7.0$ neutrinos and $19.1 \pm 3.6$ anti-neutrinos. Table 9.4 lists the number of selected neutrinos and anti-ncutrinos, scparated into up-going and down-going cvents. Figure 9.6 shows the distribution of the reconstructed muon zenith angle for selected neutrinos and anti-neutrinos in data and $\mathrm{MC}$. The expected distributions are shown for no oscillations, and for oscillations with $\Delta m_{23}^{2}=2.5 \times 10^{-3} \mathrm{cV}^{2}, \sin ^{2} 2 \theta_{23}=1.0$. The data are more consistent with oscillations although the statistical errors are large.

\subsubsection{Determination of up/down Ratio}

The atmospheric neutrino $u p /$ down ratio provides a measure of the consistency of the data with neutrino oscillations. Using the events with a well-measured direction, the measured up/down ratio is $\left(R_{u / d}\right)_{D A T A}=0.58 \pm 0.16($ stat $)$. This compares with an cxpectation of $\left(R_{u / d}\right)_{M C}=0.93 \pm 0.06(s y s)$ for no oscillations. The systematic crror in the expected up/down ratio is determined using $\mathrm{MC}$ atmospheric neutrino samples generated with alternative physics models and detector conditions (see Section 8.9). The difference between the up/down ratio calculated for the central MC sample and for each alternative MC sample is taken as the systematic error due to the choice of model. Table 9.5 lists the systematic errors calculated for cach alternative MC sample. An additional error of $5 \%$ resulting from uncertainties in the flux model is assumed. The ratio between the measured and expected up/down ratios is calculated to be:

$$
\frac{\left(R_{u / d}\right)_{D A T A}}{\left(R_{u / d}\right)_{M C}}=0.62 \pm 0.17(s t a t) \pm 0.04(s y s)
$$

This is 2.2 standard deviations away from the expectation for no oscillations.

The measured up/down ratio is compared with the expectation for a range of oscillation parameters. Figure 9.7 shows the expected up/down ratio as a function of $\Delta m_{23}^{2}$, assuming oscillations with $\sin ^{2} 2 \theta_{23}=1.0$. The up/down ratio initially falls with $\Delta m_{23}^{2}$ as the oscillation probability for up-going events increases while the oscillation proba- 
bility for down-going events remains low. The up/down ratio then rises with $\Delta m_{23}^{2}$ as the oscillation probability for down-going events increases while the oscillation probability for up-going events tends to a mean value of one half. The measured up/down ratio is plotted along with the statistical error at \pm 1 standard deviation. The measured up/down ratio is 2.1 standard deviations away from the expectation for no oscillations.

\subsubsection{Determination of $\bar{\nu}_{\mu} / \nu_{\mu}$ Ratio}

The atmospheric $\bar{\nu}_{\mu} / \nu_{\mu}$ ratio provides a measure of the consistency between neutrino and anti-neutrino oscillations. Using the events with a well-measured charge, the measured $\bar{\nu}_{\mu} / \nu_{\mu}$ ratio in data is $\left(R_{\bar{\nu} / \nu}\right)_{D A T A}=0.48 \pm 0.16($ stat $)$. This compares with an cxpectation of $\left(R_{\bar{\nu} / \nu}\right)_{M C}=0.53 \pm 0.04(s y s)$ for no oscillations. The expected $\bar{\nu}_{\mu} / \nu_{\mu}$ ratio does not vary significantly with the oscillation parameters. The systematic error in the expected $\bar{\nu}_{\mu} / \nu_{\mu}$ ratio is calculated using MC atmospheric neutrino samples generated with altcrnative physics models and detector conditions (sce Section 8.9). The difference between the $\bar{\nu}_{\mu} / \nu_{\mu}$ ratio calculated for the central MC sample and for each alternative MC sample is taken as the systematic error due to the choice of model. Table 9.6 lists the systematic crors calculated for each alternative MC sample. An additional crror of $5 \%$ resulting from uncertainties in the flux model is assumed. The ratio between the measured and expected $\bar{\nu}_{\mu} / \nu_{\mu}$ ratios is calculated to be:

$$
\frac{\left(R_{\bar{\nu} / \nu}\right)_{D A T A}}{\left(R_{\bar{\nu} / \nu}\right)_{M C}}=0.90 \pm 0.29(\text { stat }) \pm 0.06(\text { sys })
$$

The measured and expected values are consistent within errors.

In order to study the sensitivity of the $\bar{\nu}_{\mu} / \nu_{\mu}$ ratio to differences between neutrinos and anti-neutrinos, the neutrino oscillation parameters $\left(\Delta m_{23}^{2}, \sin ^{2} 2 \theta_{23}\right)$ are fixed and the anti-neutrino oscillation parameters $\left(\Delta \bar{m}_{23}^{2}, \sin ^{2} 2 \bar{\theta}_{23}\right)$ are allowed to vary. Figure 9.8 shows the expected $\bar{\nu}_{\mu} / \nu_{\mu}$ ratio as a function of $\Delta \bar{m}_{23}^{2}$, assuming oscillations with $\Delta m_{23}^{2}=2.5 \times 10^{-3} \mathrm{eV}^{2}, \sin ^{2} 2 \theta_{23}=\sin ^{2} 2 \bar{\theta}_{23}=1.0$. The expected $\bar{\nu}_{\mu} / \nu_{\mu}$ ratio falls slowly with $\Delta \bar{m}_{23}^{2}$. The measured $\bar{\nu}_{\mu} / \nu_{\mu}$ ratio is plotted along with the statistical crror at \pm 1 standard deviation. With the current statistics, the sensitivity to differences between neutrinos and anti-neutrinos is low. 


\begin{tabular}{|c|c|}
\hline Systematic effect & Error in up/down ratio \\
\hline Uncertainty in Flux Model & $5 \%$ \\
Hadronic Model $($ Gheisha) & $3 \%$ \\
QE Cross-section $( \pm 20 \%)$ & $<1 \%$ \\
Timing Resolution $( \pm 0.1 n s)$ & $1 \%$ \\
Encrgy Calibration $( \pm 5 \%)$ & $1 \%$ \\
Cosmic Muon Normalization $( \pm 10 \%)$ & $<1 \%$ \\
\hline Total & $6 \%$ \\
\hline
\end{tabular}

Table 9.5: Systematic uncertainties in the up/down ratio determined using $M C$ atmospheric neutrino samples generated with alternative physics models and detector conditions.

\begin{tabular}{|c|c|}
\hline Systematic cffect & Error in $\bar{\nu}_{\mu} / \nu_{\mu}$ ratio \\
\hline Uncertainty in Flux Model & $5 \%$ \\
Hadronic Model (Gheisha) & $4 \%$ \\
QE Cross-section $( \pm 20 \%)$ & $3 \%$ \\
Timing Resolution $( \pm 0.1 n s)$ & $1 \%$ \\
Energy Calibration $( \pm 5 \%)$ & $1 \%$ \\
Cosmic Muon Normalization $( \pm 10 \%)$ & $<1 \%$ \\
\hline Total & $7 \%$ \\
\hline
\end{tabular}

Table 9.6: Systematic uncertainties in the $\bar{\nu}_{\mu} / \nu_{\mu}$ ratio determined using $M C$ atmospheric neutrino samples generated with alternative physics models and detector conditions. 


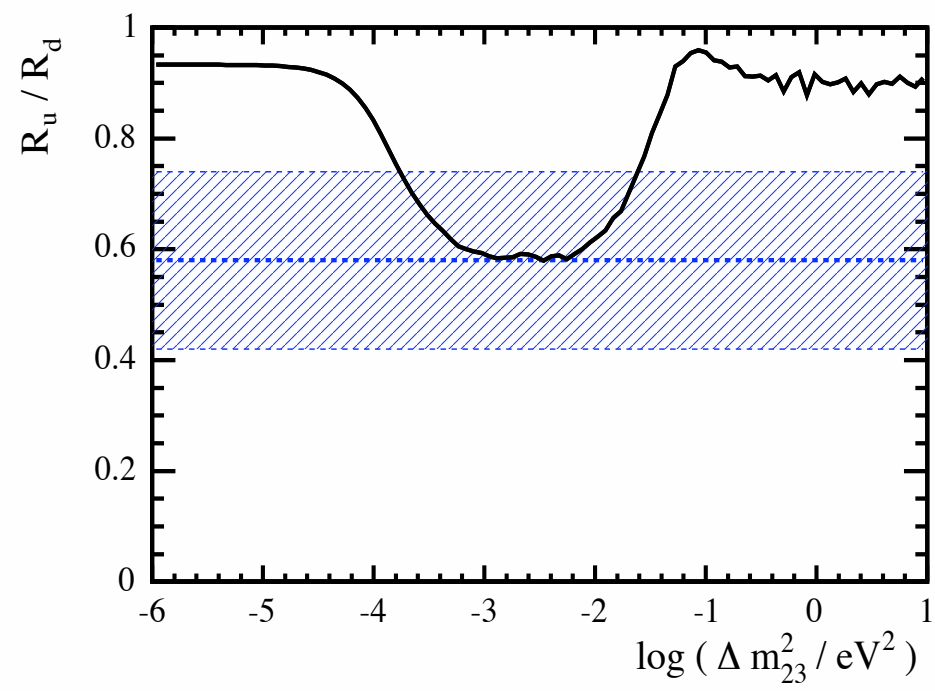

Figure 9.7: The up/down ratio as a function of $\Delta m_{23}^{2}$, assuming oscillations with $\sin ^{2} 2 \theta_{23}=1$. The solid line represents the expected up/down ratio; the dashed blue line represents the measured up/down ratio; the hatched region represents the statistical errors at \pm 1 standard deviations.

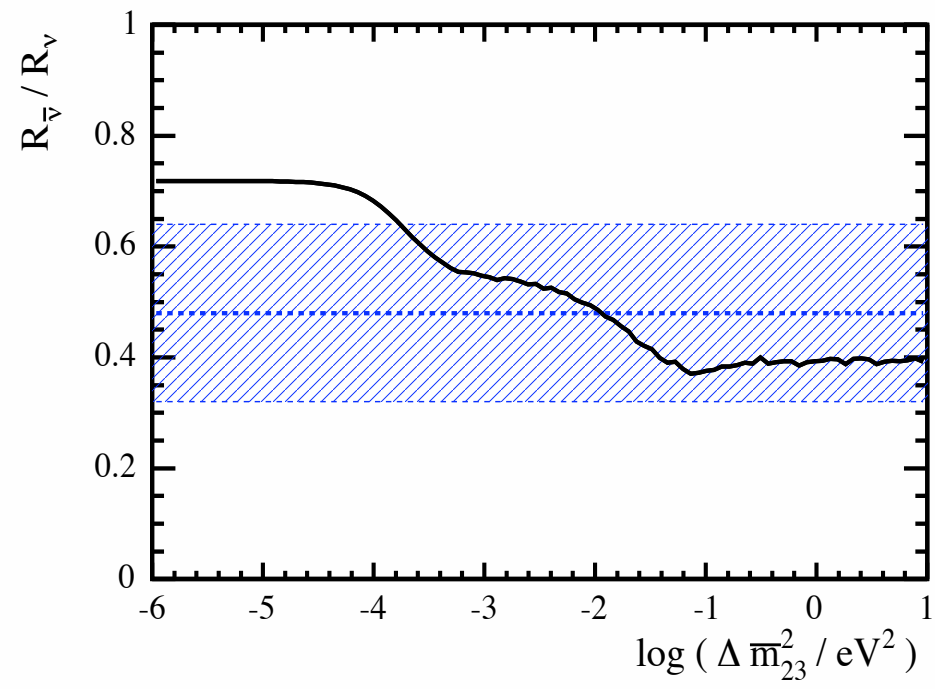

Figure 9.8: The $\bar{\nu}_{\mu} / \nu_{\mu}$ ratio as a function of $\Delta \bar{m}_{23}^{2}$, assuming oscillations with $\Delta m_{23}^{2}=2.5 \times 10^{-3} \mathrm{eV}^{2}, \sin ^{2} 2 \theta_{23}=\sin ^{2} 2 \bar{\theta}_{23}=1$. The solid line represents the expected $\bar{\nu}_{\mu} / \nu_{\mu}$ ratio; the dashed blue line represents the measured $\bar{\nu}_{\mu} / \nu_{\mu}$ ratio; the hatched region represents the statistical error at \pm 1 standard deviations. 


\subsection{Fits to Neutrino Oscillations}

A maximum likelihood method is used to fit the oscillation parameters $\Delta m_{23}^{2}$ and $\sin ^{2} 2 \theta_{23}$ assuming two-flavour $\nu_{\mu} \leftrightarrow \nu_{\tau}$ oscillations. In this approximation the probability of neutrino oscillations is given as follows:

$$
P\left(\nu_{\mu} \leftrightarrow \nu_{\tau}\right)=\sin ^{2} 2 \theta_{23} \sin ^{2}\left(\frac{1.27 \Delta m_{23}^{2} L}{E}\right)
$$

where:

$$
\begin{aligned}
& E=\text { neutrino energy }(\mathrm{GeV}) . \\
& L=\text { neutrino propagation distance }(\mathrm{km}) .
\end{aligned}
$$

The reconstructed $L / E$ distribution is used as the basis for the oscillation fit. The neutrino energy $E$ is calculated by summing the reconstructed muon momentum and reconstructed shower energy. For FC events, the muon momentum is calculated from the measured range of the muon track; for PC events, the muon momentum is calculated from the measured curvature of the muon track. The PC events are required to have a well-measured charge in order to have a well-measured energy. Figure 9.9 shows the measured and expected distributions of reconstructed energies. The neutrino propagation distance $\mathrm{L}$ is calculated by projecting the reconstructed muon direction back to a constant production height in the atmosphere as follows:

$$
L=-(R-d) \cos \theta+\sqrt{(R-d)^{2} \cos ^{2} \theta+(d+h)(2 R-d+h)}
$$

where:

$\theta=$ reconstructed muon zenith angle.

$d=$ depth of the detector $(0.7 \mathrm{~km})$.

$h=$ neutrino production height in the atmosphere $(20 \mathrm{~km})$.

$R=$ radius of the earth $(6370 \mathrm{~km})$.

Events are required to have a well-measured direction in order to have a well measured propagation distance. Since the propagation distance varies rapidly with zenith angle, events are binned in terms of $\log _{10}(L / E)$. 


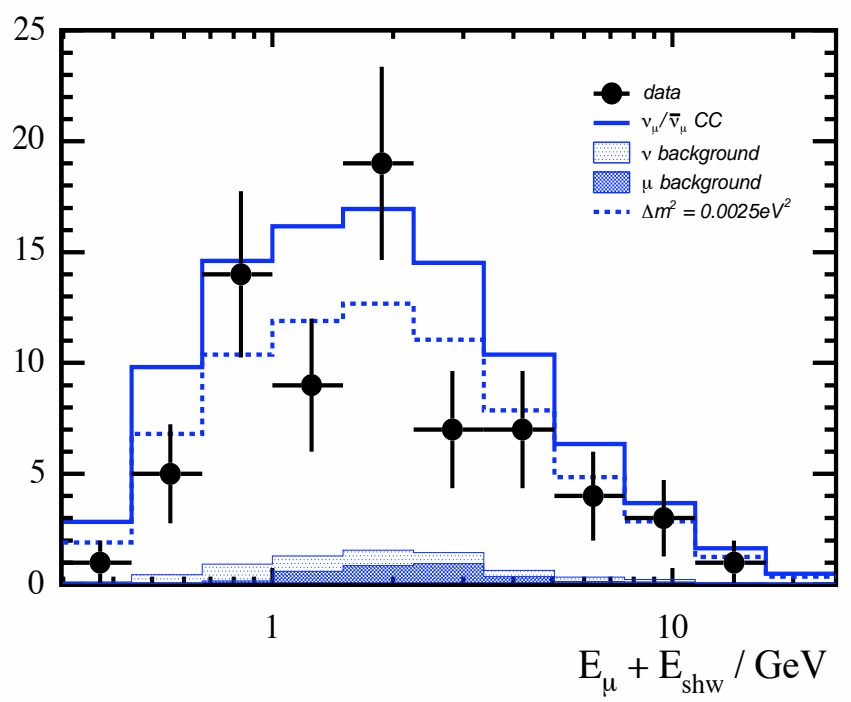

Figure 9.9: Distribution of reconstructed neutrino energies for all FC events and $P C$ events with well-measured charge. The data are represented by the points; the solid blue line represents the expectation for no oscillations; the dashed blue line represents the expectation for oscillations with the parameters $\Delta m_{23}^{2}=2.5 \times 10^{-3} \mathrm{eV}^{2}, \sin ^{2} 2 \theta_{23}=1.0$.

Figure 9.10 shows the difference between the reconstructed and true $\log _{10}(L / E)$ using the true muon direction to calculate the propagation distance. The resolution is calculated to be $\sigma_{\log (L / E)}=0.16$ for FC events and $\sigma_{\log (L / E)}=0.15$ for PC events. Figure 9.11 shows the difference between the reconstructed and true $\log _{10}(L / E)$ using the true neutrino direction to calculate the propagation distance. The $L / E$ resolution is degraded by the scattering angle between the muon and neutrino. The resolution is calculated to be $\sigma_{\log (L / E)}=0.72$ for $\mathrm{FC}$ events and $\sigma_{\log (L / E)}=0.41$ for PC events. The FC distribution has large tails due to the smaller mean energy of FC events and therefore the larger mean angle between the muons and neutrino.

Figure 9.12 shows the measured and expected distributions of $\log _{10}(L / E)$ for selected events; Figure 9.13 shows the measured and expected distributions of $\log _{10}(L / E)$ for selected neutrinos and anti-neutrinos. In both cases the expected distributions are shown for no oscillations, and for oscillations with $\Delta m_{23}^{2}=2.5 \times 10^{-3} \mathrm{eV}^{2}, \sin ^{2} 2 \theta_{23}=1.0$. The data are more consistent with oscillations although the statistical errors are large. 

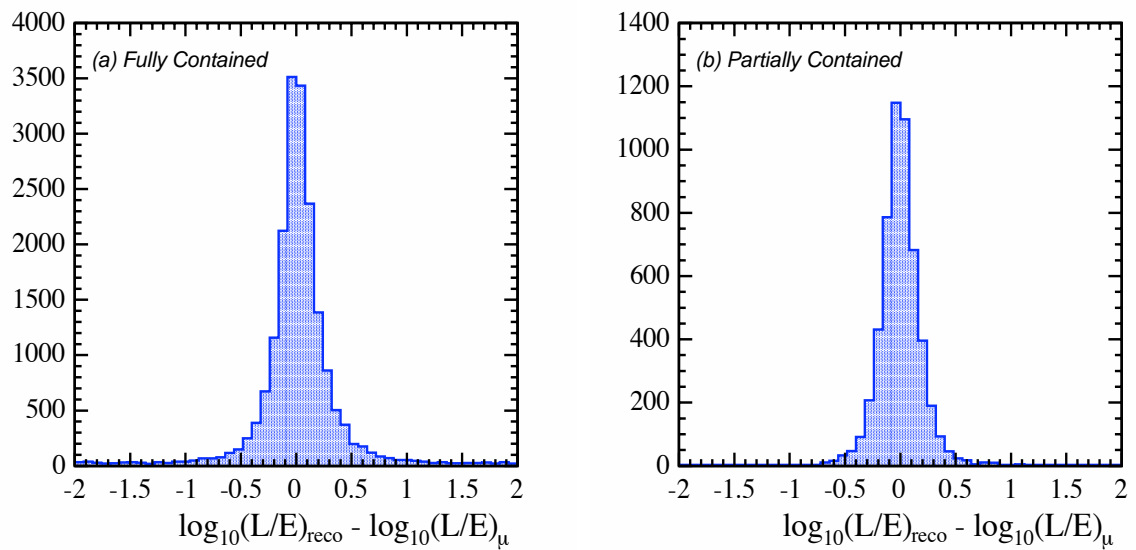

Figure 9.10: Distribution of differences between reconstructed and true values of $\log _{10}(L / E)$ for well-measured events. The true muon direction is used to calculate the propagation length. The distributions are separated into fully contained and partially contained events.
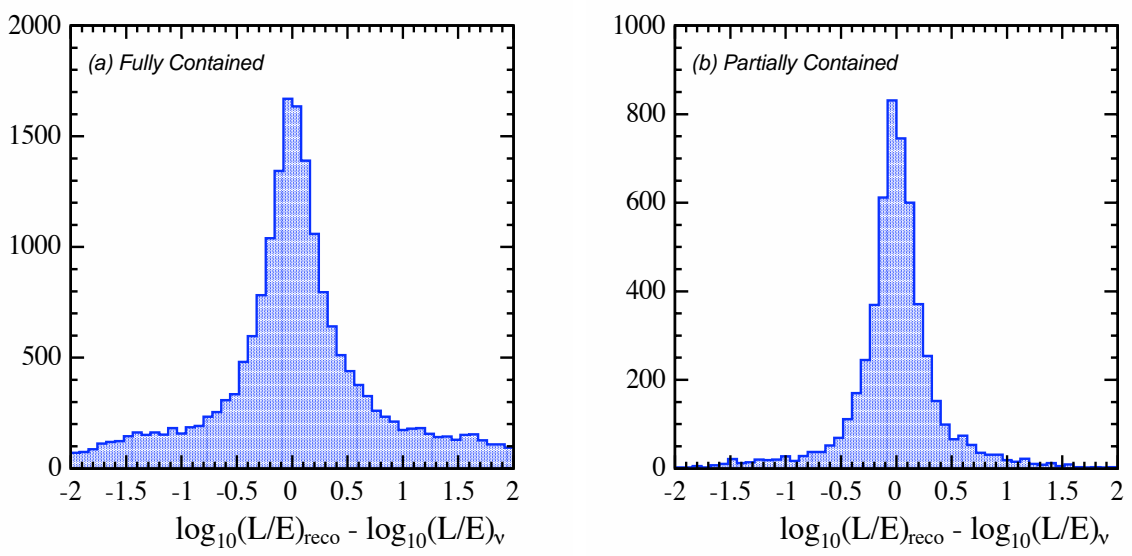

Figure 9.11: Distribution of differences between reconstructed and true values of $\log _{10}(L / E)$ for well-measured events. The true neutrino direction is used to calculate the propagation length. The distributions are separated into fully contained and partially contained events. 

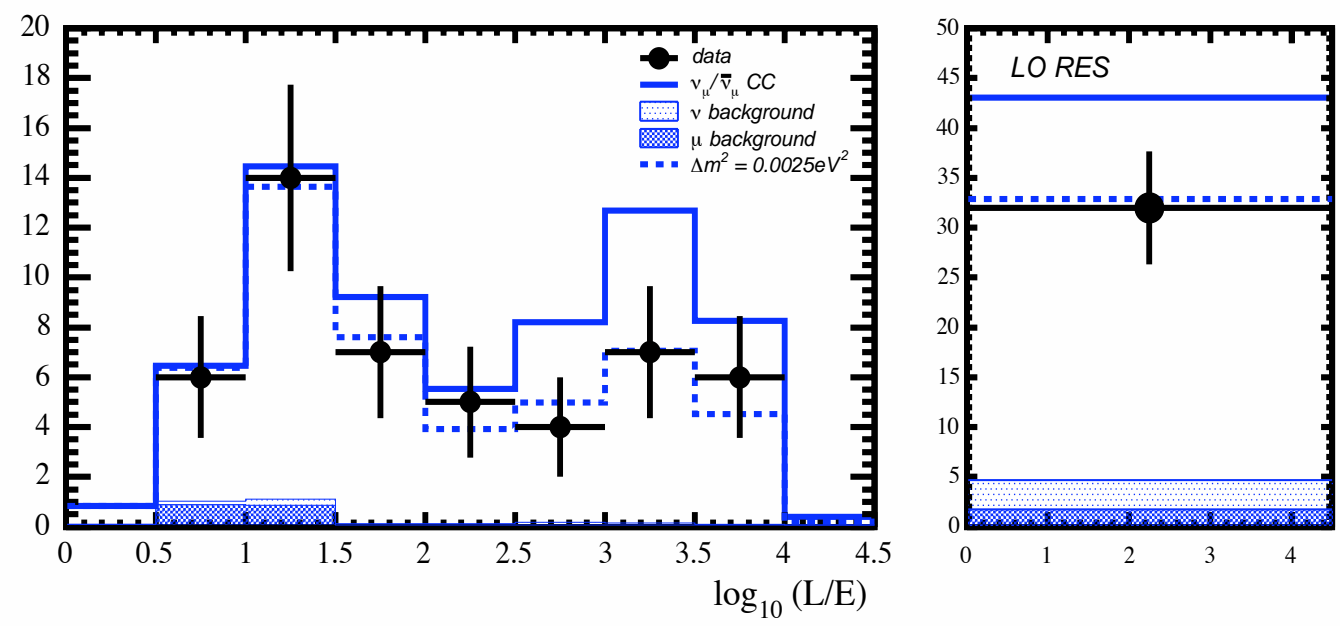

Figure 9.12: Distribution of reconstructed L/E for well-measured events (left) and low resolution events (right). The data are represented by the points; the solid blue line represents the expectation for no oscillations; the dashed blue line represents the expectation for oscillations with the parameters $\Delta m_{23}^{2}=2.5 \times 10^{-3} \mathrm{eV}^{2}, \sin ^{2} 2 \theta_{23}=1.0$.
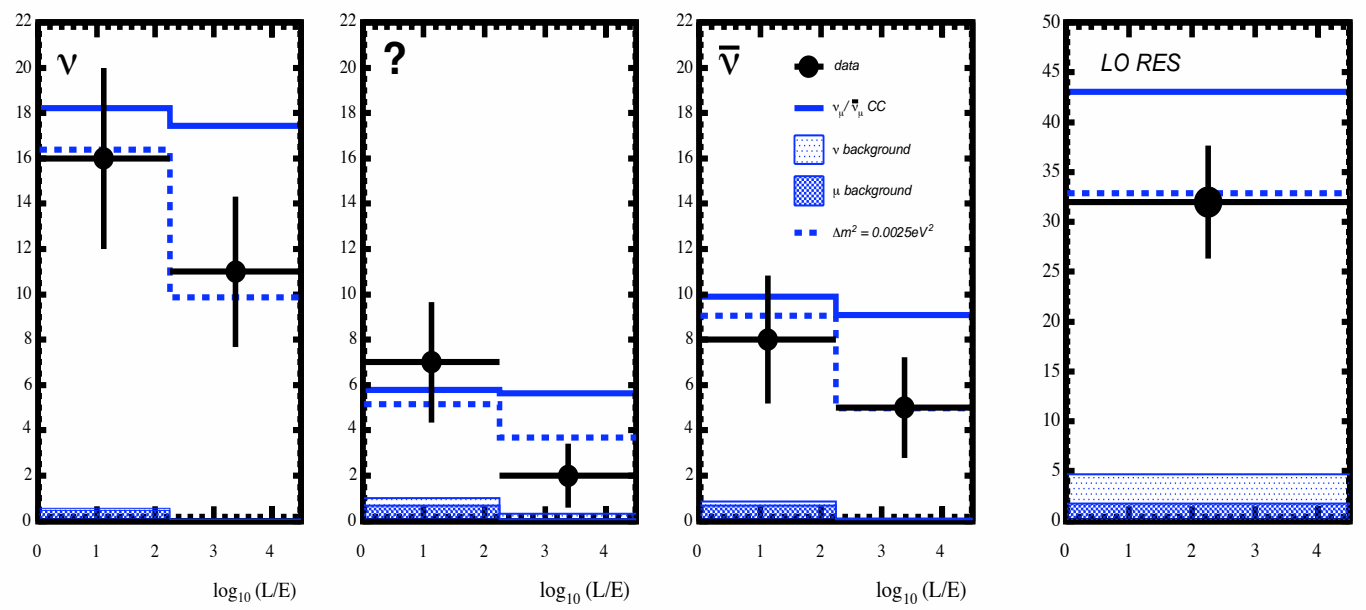

Figure 9.13: Distribution of reconstructed $L / E$ for events classified as neutrinos $\left(\nu_{\mu}\right)$, events with an ambiguous charge (?), events classified as antineutrinos $\left(\bar{\nu}_{\mu}\right)$, and low resolution events (right). The data are represented by the points; the solid blue line represents the expectation for no oscillations; the dashed blue line represents the expectation for oscillations with the parameters $\Delta m_{23}^{2}=2.5 \times 10^{-3} e V^{2}$, $\sin ^{2} 2 \theta_{23}=1.0$. 
The reconstructed $\log _{10}(L / E)$ distribution is subject to several systematic effects that must be taken into account in the oscillation fit. These effects are studied using MC atmospheric neutrino samples generated with alternative physics models and detector conditions (sec Section 8.9). Figures 9.14 (a)-(f) show the reconstructed $\log _{10}(L / E)$ distributions for the central MC sample and for a range of alternative $\mathrm{MC}$ samples assuming no oscillations. The systematic effects are discussed in detail below:

(a) To study systematic errors resulting from the choice of atmospheric neutrino flux model, the model of Barr et al [124] used in the central MC sample is replaced by the model of Battistoni et al [142]. This distorts the $\log _{10}(L / E)$ distribution.

(b) To study systematic errors resulting from the choice of hadronic interaction model, the GCALOR model [141] used in the central MC sample is replaced by the Geant 3 implementation of the GHEISHA model [143]. This is found to distort the $\log _{10}(L / E)$ distribution at high values.

(c) To study the effect of uncertainties in the neutrino interaction model, the QE cvents are re-weighted by $\pm 20 \%$. Figure 9.14(c) shows the effect of re-weighting the QE events by $+20 \%$. The $\log _{10}(L / E)$ distribution is shifted towards higher values, corresponding to lower energies where $\mathrm{QE}$ events are distributed.

(d) To study the effect of uncertainties in the cosmic muon background, the $\log _{10}(L / E)$ distribution for the background is re-weighted by $\pm 10 \%$. Figure 9.14(d) shows the effect of re-weighting the background events by $+10 \%$. The $\log _{10}(L / E)$ distribution is increased at low values where the background is distributed.

(e) To study the effect of uncertainties in the timing resolution, the resolution is varied by \pm 0.1 ns. Figure $9.14(\mathrm{e})$ shows the effect of varying the resolution by $+0.1 \mathrm{~ns}$. The $\log _{10}(L / E)$ distribution is shifted slightly towards lower values since a larger fraction of low energy events have an ambiguous direction.

(f) To study the effect of uncertainties in the detector energy calibration, the light output is varied by $\pm 5 \%$. Figure $9.14(\mathrm{f})$ shows the effect of changing the light output by $+5 \%$. The $\log _{10}(L / E)$ distribution is shifted towards lower values since the mean reconstructed energy of neutrino events is increased. 
(a)

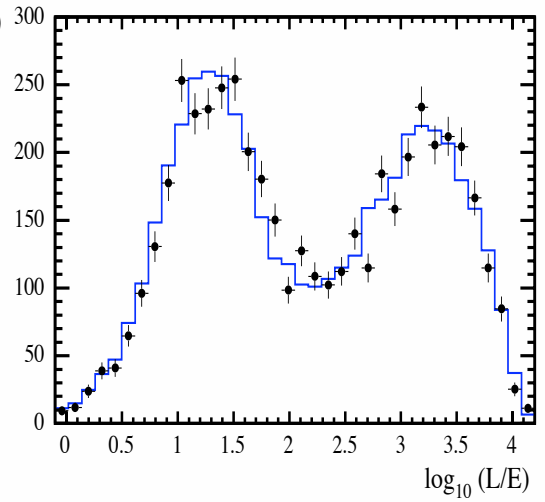

(c)

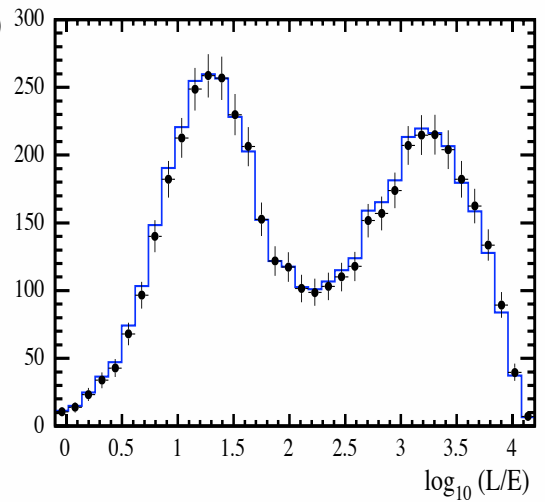

(e)

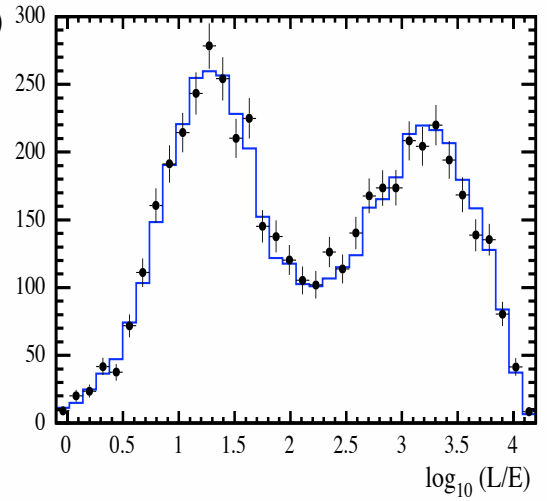

(b)

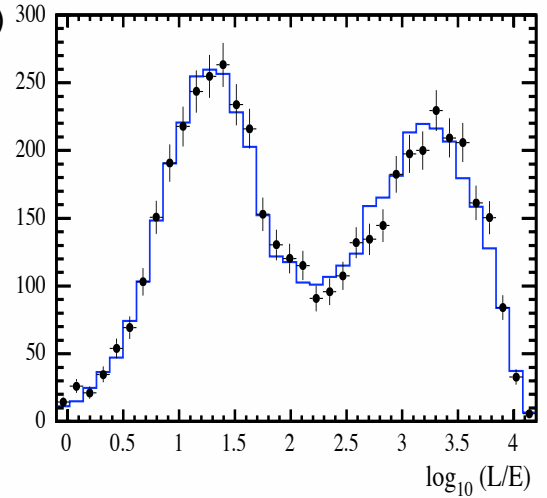

(d)

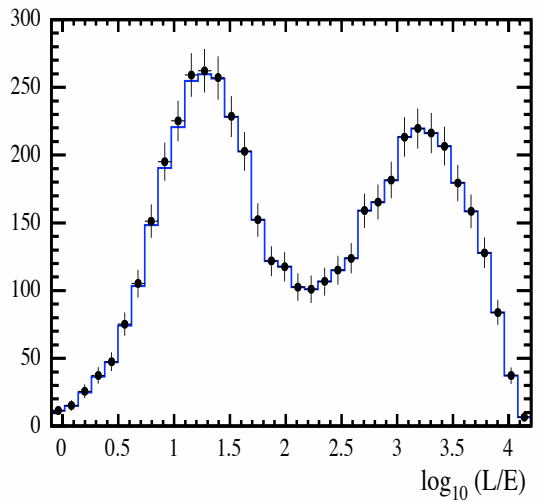

(f)

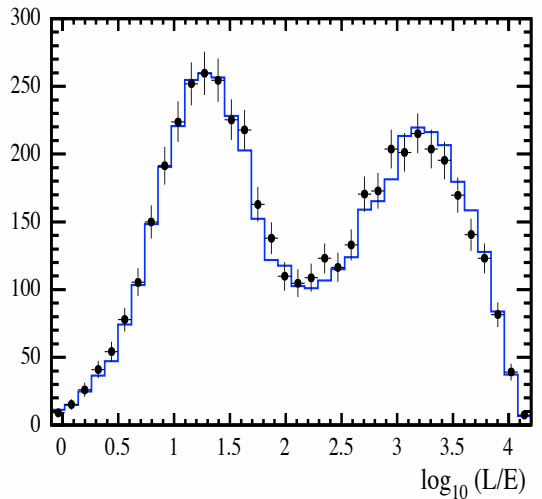

Figure 9.14: Comparison between reconstructed $L / E$ distributions for central MC sample (solid blue lines) and alternative MC samples (points). The alternative $M C$ samples are generated using the following models: (a) Battistoni flux model in place of Barr model. (b) GHEISHA hadronic model in place of GCALOR model. (c) QE events re-weighted by $+20 \%$. (d) cosmic muon background re-weighted by $+10 \%$. (e) timing resolution smeared by $+0.1 \mathrm{~ns}$. (f) detector response increased by $+5 \%$. 


\begin{tabular}{|c|c|}
\hline Systematic Effect & $-\Delta \ln \mathrm{L}$ \\
\hline$\nu_{\mu} / \bar{\nu}_{\mu}$ Normalization $(+20 \%)$ & 1.93 \\
$\nu_{\mu} / \bar{\nu}_{\mu}$ Normalization $(-20 \%)$ & 2.53 \\
$u p /$ down Ratio $( \pm 5 \%)$ & 0.03 \\
$\bar{\nu}_{\mu} / \nu_{\mu}$ Ratio $( \pm 5 \%)$ & 0.04 \\
Flux Model (Battistoni) & 0.08 \\
Hadronic Model (Gheisha) & 0.09 \\
QE Cross-Scction $( \pm 20 \%)$ & 0.05 \\
Timing Resolution $( \pm 0.1 n s)$ & 0.06 \\
Energy Calibration $( \pm 5 \%)$ & 0.05 \\
Cosmic Muon Normalization $( \pm 10 \%)$ & $<0.01$ \\
\hline
\end{tabular}

Table 9.7: The log-likelihood difference between the $\log _{10} L / E$ distribution for the central $M C$ atmospheric neutrino sample and alternative $M C$ samples generated using different physics models and detector conditions.

Only systematic errors that are significant compared to the statistical errors in the data are included in the oscillation fit. The significance of each systematic effect is estimated using the following log-likelihood function:

$$
-\Delta \ln \mathcal{L}=\sum_{i=0}^{n}\left(X_{i}-N_{i} \ln X_{i}\right)-\sum_{i=0}^{n}\left(N_{i}-N_{i} \ln N_{i}\right)
$$

where:

$N_{i}=$ expected number of events in $i^{\text {th }}$ bin for central MC sample.

$X_{i}=$ cxpected number of events in $i^{\text {th }}$ bin for alternative MC sample.

The $\log _{10}(L / E)$ distributions are normalized to the exposure of the data and are binned in the same way as the distributions used for the oscillation fit (see Figure 9.12). Equation 9.3 is used to calculate the difference in log-likelihood between the central MC sample and each alternative MC sample. Table 9.7 lists the values of $-\Delta \ln \mathcal{L}$ calculated for each systematic effect considered. This compares with a value of $-\Delta \ln \mathcal{L}=1.2$ resulting from Gaussian errors at \pm 1 standard deviation. Only the uncertainty in the atmospheric neutrino normalization is significant at this level. 


\subsubsection{Combined $\nu_{\mu} / \bar{\nu}_{\mu}$ Oscillation Fit}

A combined neutrino and anti-neutrino oscillation fit is first performed on the data. The selected atmospheric neutrino events are scparated into a high resolution sample containing events with well-measured $L / E$, and a low resolution sample containing all other events. The high resolution sample is binned in terms of $\log _{10}(L / E)$ while the low resolution sample is contained in a single bin (sec Figure 9.12)

The oscillation fit is performed on a $140 \times 140$ grid in $\left(\log _{10} \Delta m_{23}^{2}, \sin ^{2} 2 \theta_{23}\right)$ parameter space over the range $\left(-6<\log _{10} \Delta m_{23}^{2}<1,0<\sin ^{2} 2 \theta_{23}<1\right)$. Neutrinos and antineutrinos assumed to have the same oscillation parameters. The expected $\log _{10}(L / E)$ distribution is generated at each grid point and the negative log-likelihood $-\ln \mathcal{L}$ for the observed $\log _{10}(L / E)$ distribution is calculated as follows:

$$
-\ln \mathcal{L}=\sum_{i=0}^{n}\left(X_{i}-N_{i} \ln X_{i}\right)+\frac{\alpha^{2}}{2 \sigma_{\alpha}^{2}}
$$

where:

$N_{i}=$ observed number of events in $i^{t h}$ bin.

$X_{i}=$ expected number of events in $i^{\text {th }}$ bin.

$\alpha=$ variation in atmospheric neutrino normalization.

$\sigma_{\alpha}=$ systematic crror in atmospheric neutrino normalization.

The expectation $X_{i}$ at each grid point is given by:

$$
X_{i}=(1+\alpha) S_{i}\left(\Delta m_{23}^{2}, \sin ^{2} 2 \theta_{23}\right)+B_{i}
$$

where:

$S_{i}=$ expected number of atmospheric neutrino events in $i^{\text {th }}$ bin.

$B_{i}=$ expected number of cosmic muon events in $i^{\text {th }}$ bin.

The systematic error in the atmospheric neutrino normalization is accommodated in the oscillation fit by allowing the expected $\log _{10}(L / E)$ distribution for atmospheric neutrinos to vary by an amount $\alpha$ and adding a Gaussian error term $\alpha^{2} / 2 \sigma_{\alpha}^{2}$ in the log-likelihood. The value of $\sigma_{\alpha}$ is taken to be $20 \%$ (see Section 8.9). The log-likelihood is minimized with respect to the parameter $\alpha$ at each grid point. 

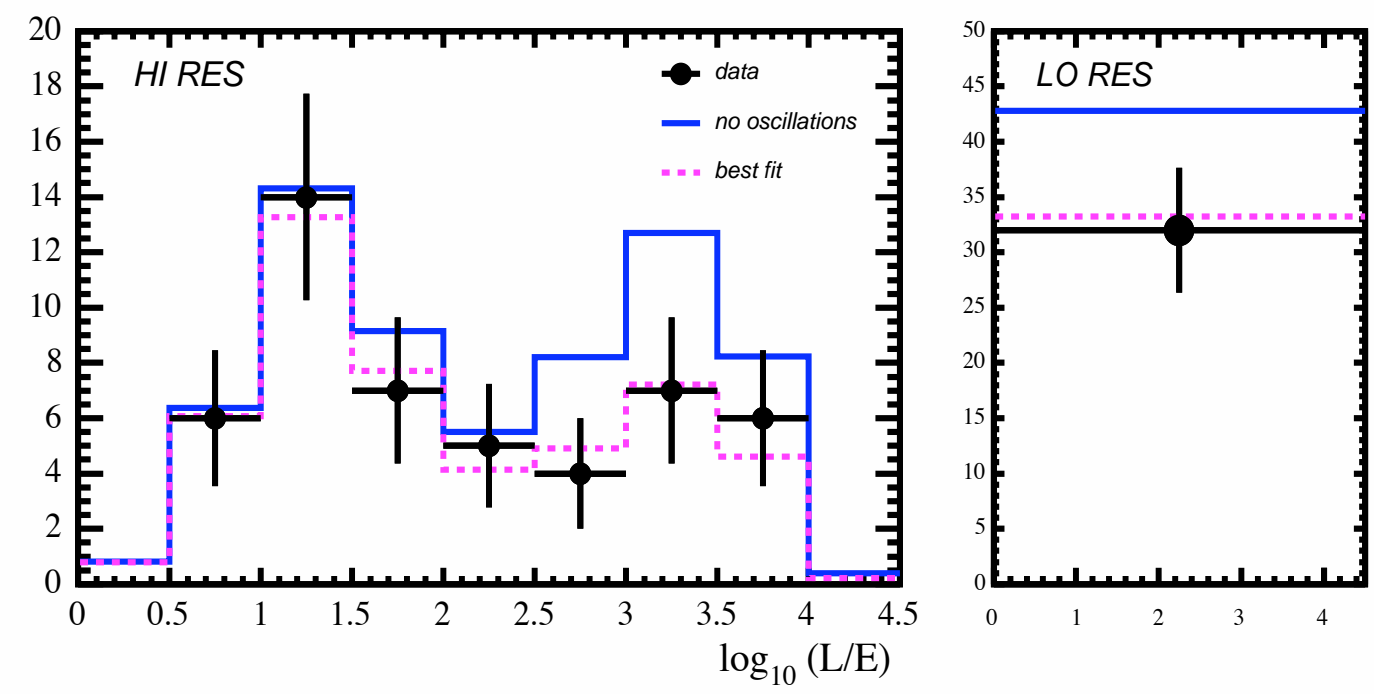

Figure 9.15: Distribution of reconstructed $L / E$ for well-measured events (left) and low resolution events (right). The data are represented by the points; the solid blue line represents the expectation for no oscillations; the dashed line represents the expectation for the best fit two-flavour oscillation parameters $\Delta m_{23}^{2}=1.3 \times 10^{-3} \mathrm{eV}^{2}, \sin ^{2} 2 \theta_{23}=0.90$.

The minimum log-likelihood occurs at $\left(\Delta m_{23}^{2}=1.3 \times 10^{-3} \mathrm{eV}^{2}, \sin ^{2} 2 \theta_{23}=0.90\right)$. The best fit atmospheric neutrino normalization at this point is $1+\alpha=0.96$. Figure 9.15 shows the distributions of $\log _{10}(L / E)$ for the data and the best fit oscillation parameters. The confidence limits on the oscillation parameters are determined by calculating the difference in the log-likelihood $-\Delta \ln \mathcal{L}$ at each grid point relative to the best fit point. In the limit of Gaussian errors, the $(68 \%, 90 \%, 99 \%)$ confidence limits are given by $-\Delta \ln \mathcal{L}=(1.2,2.3,4.6)$. Figure 9.16 shows the $68 \%$ and $90 \%$ confidence limits obtained using this method. The data are consistent with a wide range of oscillation parameters, including the results from Super-Kamiokande [51] [52] and K2K [58] (see Section 2.3). Only a small region of parameter space is disfavoured at $90 \%$ confidence. The null oscillation hypothesis is disfavoured at $79 \%$ confidence. Since the best fit point is located close to the physical boundary and the current statistics are low, it is likely that the Gaussian approximation provides an incomplete coverage of the confidence limits. However, more sophisticated techniques of constructing the confidence limits have been found to produce similar results [149]. 


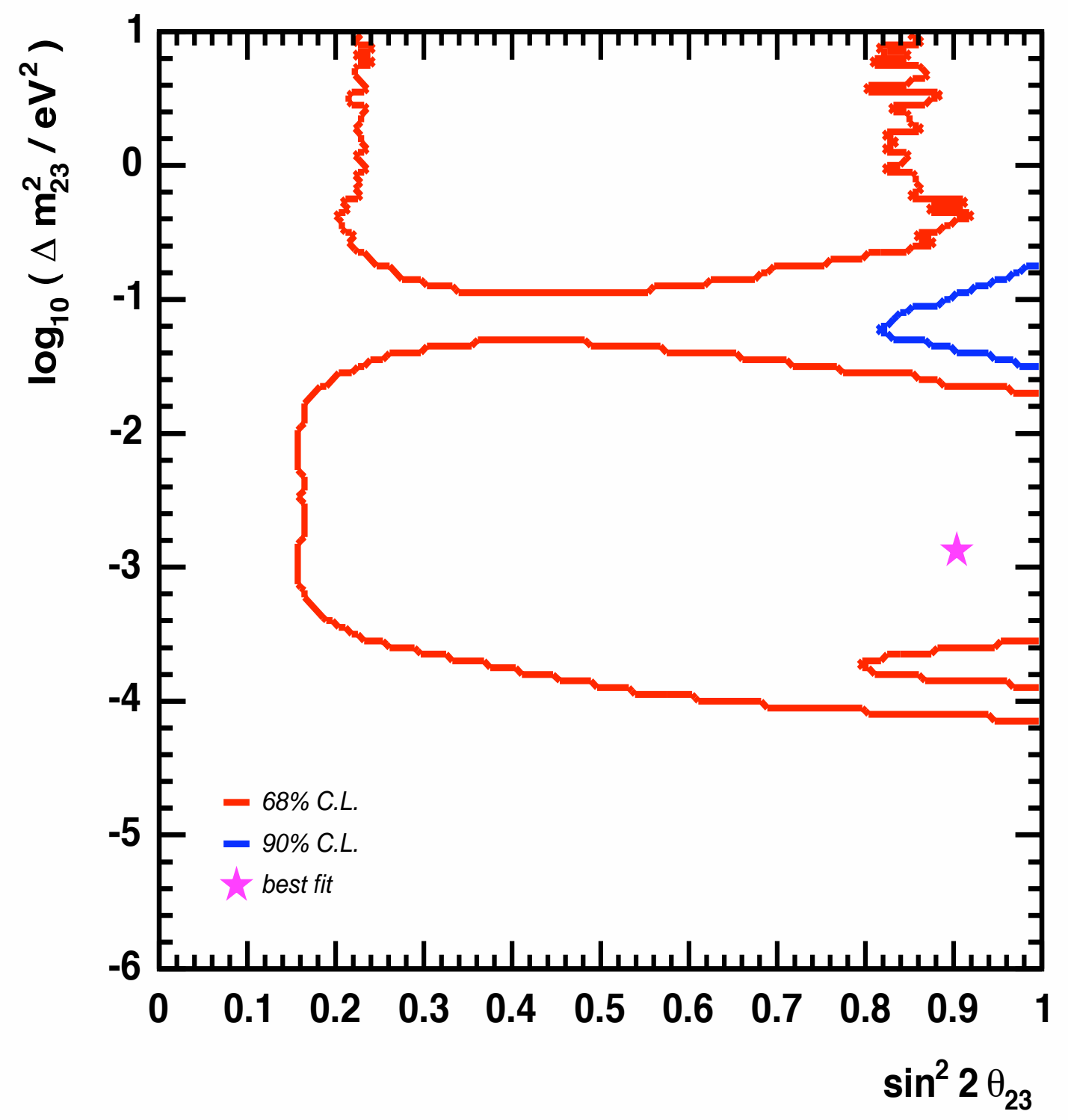

Figure 9.16: The 68\% and 90\% confidence limits obtained for the oscillation parameters $\Delta m_{23}^{2}$ and $\sin ^{2} 2 \theta_{23}$. The best fit point is indicated by the star. 


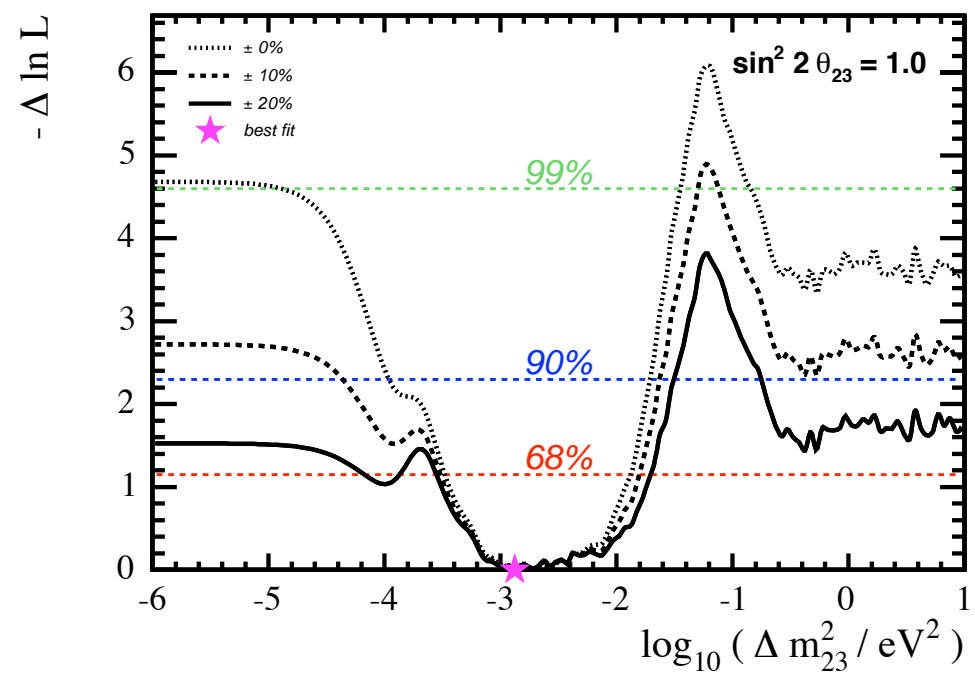

Figure 9.17: The distribution of $-\Delta \ln \mathcal{L}$ as a function of $\Delta m_{23}^{2}$ for the case where $\sin ^{2} 2 \theta_{23}=1.0$, assuming systematic errors in the atmospheric neutrino normalization of $0 \%$ (solid line), 10\% (dashed line), and $20 \%$ (dotted line). The best fit point is indicated by the star.

Figure 9.17 shows the distributions of $-\Delta \ln \mathcal{L}$ as a function of $\Delta m_{23}^{2}$ assuming $\sin ^{2} 2 \theta_{23}=1.0$, and systematic errors of $0 \%, 10 \%$ and $20 \%$ in the normalization. Each distribution has a broad minimum around the best fit point. The distributions rise sharply at either side of this minimum. The large peak at $\log _{10} \Delta m_{23}^{2}=-1.2$ corresponds to the point where the expected oscillation maximum coincides with the first peak of the $\log _{10}(L / E)$ distribution. The small peak at $\log _{10} \Delta m_{23}^{2}=-3.6$ corresponds to the point where the expected oscillation maximum coincides with the best fit oscillation minimum. The flat regions at $\log _{10} \Delta m_{23}^{2}<-5$ and $\log _{10} \Delta m_{23}^{2}>0$ correspond to the regions where the expected oscillation maximum falls outside the $\log _{10}(L / E)$ distribution.

The systematic error in the normalization has a significant effect on the calculated confidence limits. If the oscillation fit is performed assuming a fixed normalization, the null oscillation hypothesis is disfavoured at $>99 \%$ confidence. One way of reducing the uncertainty in the normalization would be to use the atmospheric neutrino flux measured by the Soudan 2 experiment which was located at the same depth and geomagnetic latitude as the MINOS Far Detector. By analysing atmospheric $\nu_{e} / \bar{\nu}_{e}$ data, Soudan 2 has determined the atmospheric neutrino normalization to within $10 \%$ [150]. 


\subsubsection{Separated $\nu_{\mu} / \bar{\nu}_{\mu}$ Oscillation Fit}

MINOS is the first underground experiment to make separate observations of atmospheric neutrinos and anti-neutrinos. A separate neutrino and anti-neutrino oscillation fit is performed on the data allowing neutrinos and anti-neutrinos to have different oscillation parameters. The selected events are divided into high resolution samples containing neutrinos, anti-neutrinos and events with well-measured $L / E$ but ambiguous charge; and a low resolution sample containing all other events. The high resolution samples are binned in terms of $\log _{10}(L / E)$ while the low resolution sample is contained in a single bin (see Figure 9.13).

The oscillations of neutrinos and anti-neutrinos are characterized by independent parameters $\left(\Delta m_{23}^{2}, \sin ^{2} 2 \theta_{23}\right)$ and $\left(\Delta \bar{m}_{23}^{2}, \sin ^{2} 2 \bar{\theta}_{23}\right)$. In order to study the sensitivity of the data to differences in the oscillations of neutrinos and anti-neutrinos, a fit is applied to the parameters $\Delta m_{23}^{2}$ and $\Delta \bar{m}_{23}^{2}$, assuming $\sin ^{2} 2 \theta_{23}=\sin ^{2} 2 \bar{\theta}_{23}=1$. The oscillation fit is performed on a $140 \times 140$ grid in $\left(\log _{10} \Delta m_{23}^{2}, \log _{10} \Delta \bar{m}_{23}^{2}\right)$ parameter space for the range $\left(-6<\log _{10} \Delta m_{23}^{2}<1,-6<\log _{10} \Delta \bar{m}_{23}^{2}<1\right)$. The expected $\log _{10}(L / E)$ distribution is generated at each grid point and the negative log-likelihood for the observed $\log _{10}(L / E)$ distribution is calculated using Equation 9.4. The systematic error in the atmospheric neutrino normalization is accommodated by allowing the expected $\log _{10}(L / E)$ distributions to vary with a systematic uncertainty of $20 \%$ in the same way as for the combined oscillation fit.

The minimum log-likelihood occurs at $\left(\Delta m_{23}^{2}=3.0 \times 10^{-4} \mathrm{cV}^{2}, \Delta \bar{m}_{23}^{2}=6.0 \times\right.$ $10^{-3} \mathrm{eV}^{2}$ ). The best fit atmospheric neutrino normalization at this point is $1+\alpha=0.96$. Figure 9.18 shows the distributions of $\log _{10}(L / E)$ for the data and the best fit oscillation parameters. The confidence limits on the oscillation parameters are calculated in the same way as for the combined oscillation fit. Figure 9.19 shows the $68 \%$ and $90 \%$ confidence limits obtained using this method. With the current statistics, the sensitivity to differences in the oscillations of neutrinos and anti-neutrinos is low. The data is consistent with oscillations of neutrino and anti-neutrino with the same parameters over a large range of $\Delta m_{23}^{2}$ and $\Delta \bar{m}_{23}^{2}$ values. Only the regions of parameter space where the oscillation parameters differ by a number of orders of magnitude are disfavoured at $90 \%$. For neutrino ocillations with $\Delta m_{23}^{2}=2 . \bar{\delta} \times 10^{-3} \mathrm{eV}^{2}$, the entire range of anti-neutrino oscillation parameters is within the $68 \%$ confidence limit. 

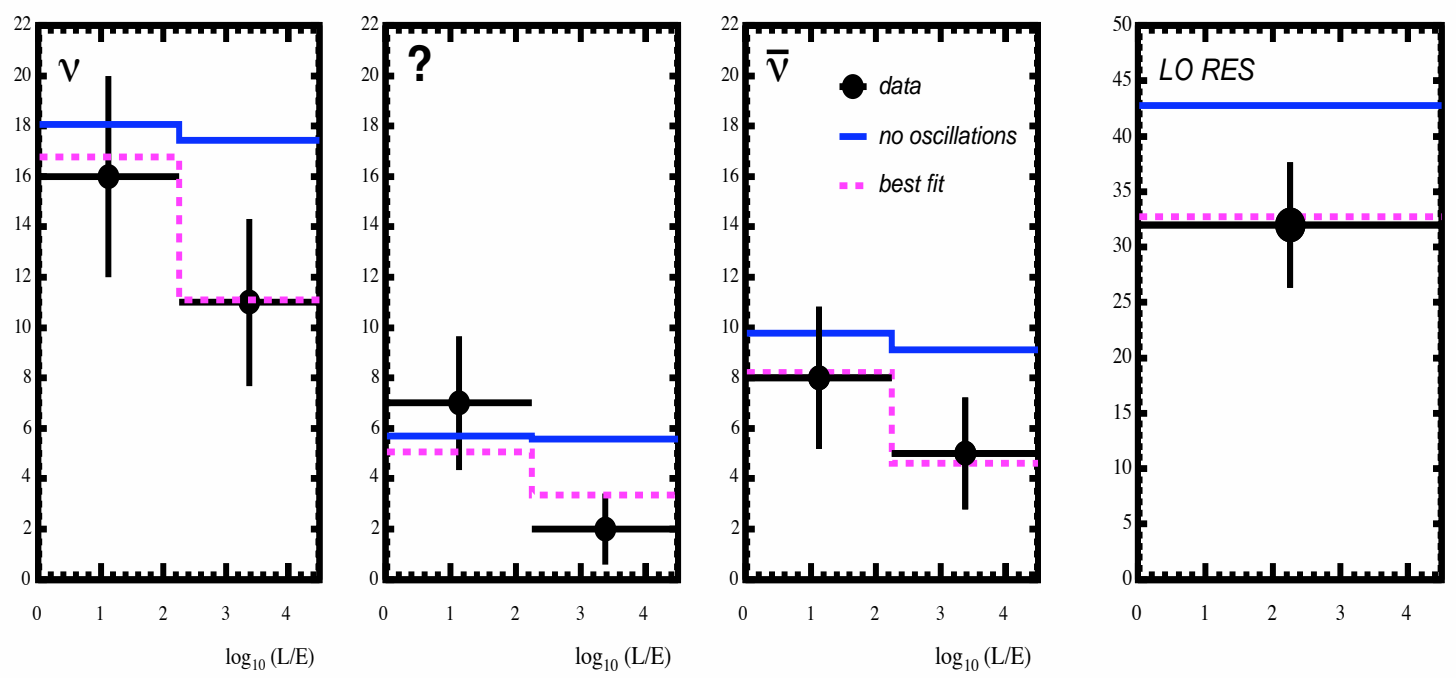

Figure 9.18: Distribution of reconstructed $L / E$ for events classified as neutrinos $\left(\nu_{\mu}\right)$, events with an ambiguous charge (?), events classified as antineutrinos $\left(\bar{\nu}_{\mu}\right)$, and low resolution events (right). The data are represented by the points; the solid blue line represents the expectation for no oscillations; the dashed line represents the expectation for the best fit oscillation parameters $\Delta m_{23}^{2}=3.0 \times 10^{-4} \mathrm{eV}^{2}, \Delta \bar{m}_{23}^{2}=6.0 \times 10^{-3} \mathrm{eV}^{2}$. 


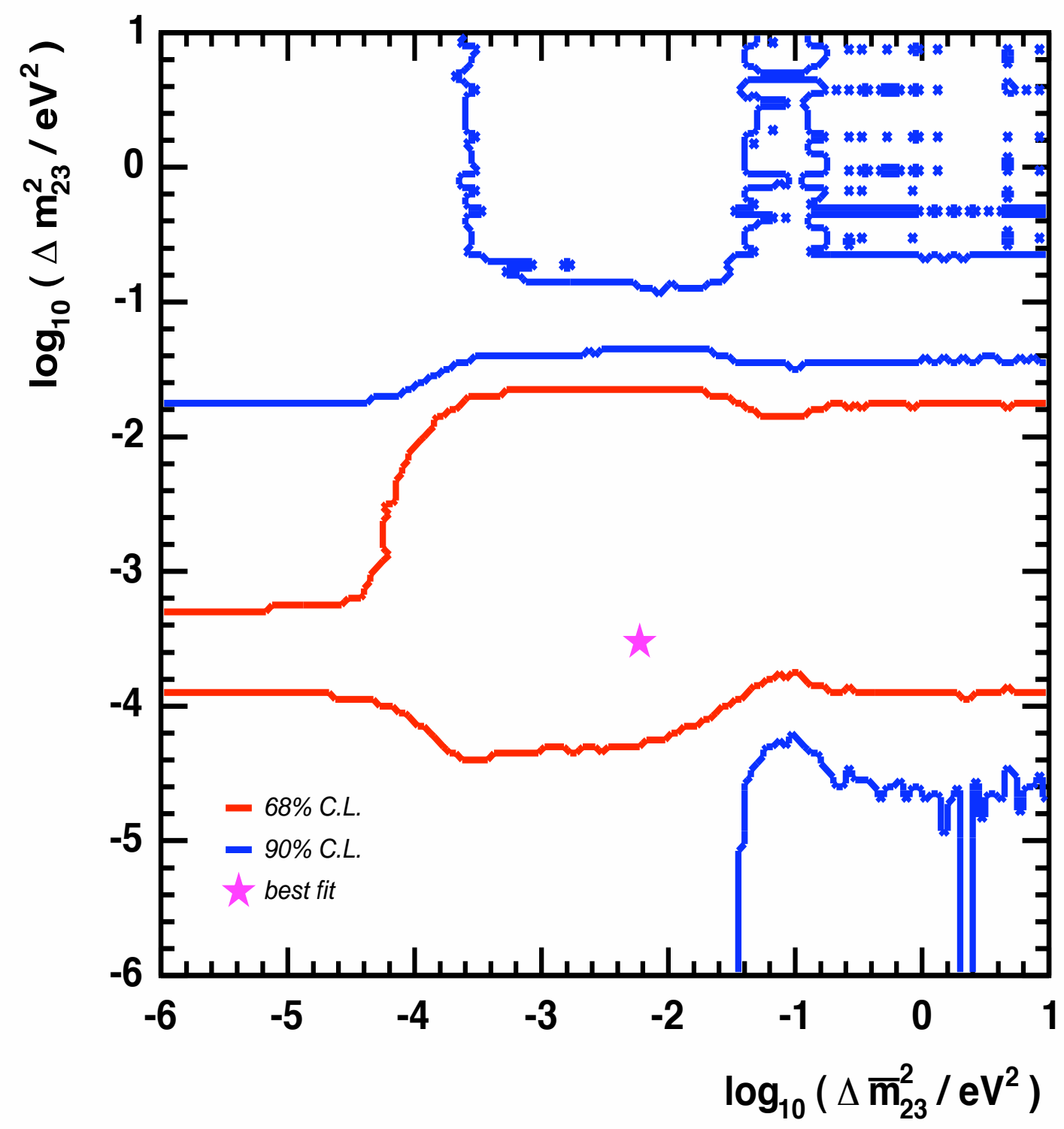

Figure 9.19: The 68\% and 90\% confidence limits obtained for the oscillation parameters $\Delta m_{23}^{2}$ and $\Delta \bar{m}_{23}^{2}$. The best fit point is indicated by the star. 


\subsection{Projected 25 kT-Yr Sensitivity}

The current sensitivity to atmospheric neutrino oscillations is limited by low statistics. In order to study the future sensitivity of the MINOS atmospheric neutrino analysis, the projected sensitivity of the combined and separated $\nu_{\mu} / \bar{\nu}_{\mu}$ oscillation fits is calculated for a detector exposure of $25 \mathrm{kT}$-Yrs (approximately 5 years detector running).

The projected sensitivity is determined by generating a series of mock experiments for a given set of oscillation parameters, and then applying the combined and scparated oscillation fits to each experiment. A total of 100 experiments are generated for each given set of oscillation parameters. The combined oscillation fits are performed on a $140 \times 140$ grid in $\left(\log _{10} \Delta m_{23}^{2}, \sin ^{2} 2 \theta_{23}\right)$ space, and the separated oscillation fits are performed on a $140 \times 140$ grid in $\left(\log _{10} \Delta m_{23}^{2}, \log _{10} \Delta \bar{m}_{23}^{2}\right)$ space. The expected $\log _{10}(L / E)$ distribution is generated at each grid point and the negative log-likelihood for the observed $\log _{10}(L / E)$ distribution is calculated for each experiment. The best fit oscillation parameters are given by the minimum log-likelihood. A systematic uncertainty of $20 \%$ in the atmospheric neutrino normalization is included in the fits as described above. At this level of statistics, other systematic effects are expected to be significant in the oscillation fits. However, it is anticipated that both the systematic errors and the techniques used to reconstruct and select atmospheric neutrino events will also improve.

For each experiment, the difference in likelihood $-\Delta \ln \mathcal{L}$ relative to the best fit is calculated at each grid point. The projected confidence limits on the oscillation parameters are determined be calculating the mean difference in likelihood $\langle-\Delta \ln \mathcal{L}\rangle$ at each grid point. In the limit of Gaussian errors, the $(68 \%, 90 \%, 99 \%)$ confidence limits are given by $\langle-\Delta \ln \mathcal{L}\rangle=(1.2,2.3,4.6)$. Figure 9.20 shows the projected sensitivity of the combined fit for the input parameters $\left(\Delta m_{23}^{2}=2.5 \times 10^{-3} \mathrm{cV}^{2}, \sin ^{2} 2 \theta_{23}=1.0\right)$. The oscillation parameters are determined to be in the range $4 \times 10^{-1}<\Delta m_{23}^{2}<2 \times 10^{-2} \mathrm{eV}^{2}$, $\sin ^{2} \theta_{23}>0.5$ at the $90 \%$ confidence level. Figure 9.21 shows the projected sensitivity of the separated fit for the input parameters $\left(\Delta m_{23}^{2}=\Delta \bar{m}_{23}^{2}=2.5 \times 10^{-3} \mathrm{eV}^{2}\right)$, assuming $\left(\sin ^{2} 2 \theta_{23}=\sin ^{2} 2 \bar{\theta}_{23}=1.0\right)$. Figure 9.22 shows the projected sensitivity for the input parameters $\left(\Delta m_{23}^{2}=2.5 \times 10^{-3} \mathrm{cV}^{2}, \Delta \bar{m}_{23}^{2}=10^{-5}, 10^{-4}, 10^{-2}, 10^{-1} \mathrm{cV}^{2}\right)$, assuming $\left(\sin ^{2} 2 \theta_{23}=\sin ^{2} 2 \bar{\theta}_{23}=1.0\right)$. This suggests that differences of 1 or 2 orders of magnitude between $\Delta m_{23}^{2}$ and $\Delta \bar{m}_{23}^{2}$ can be resolved. 


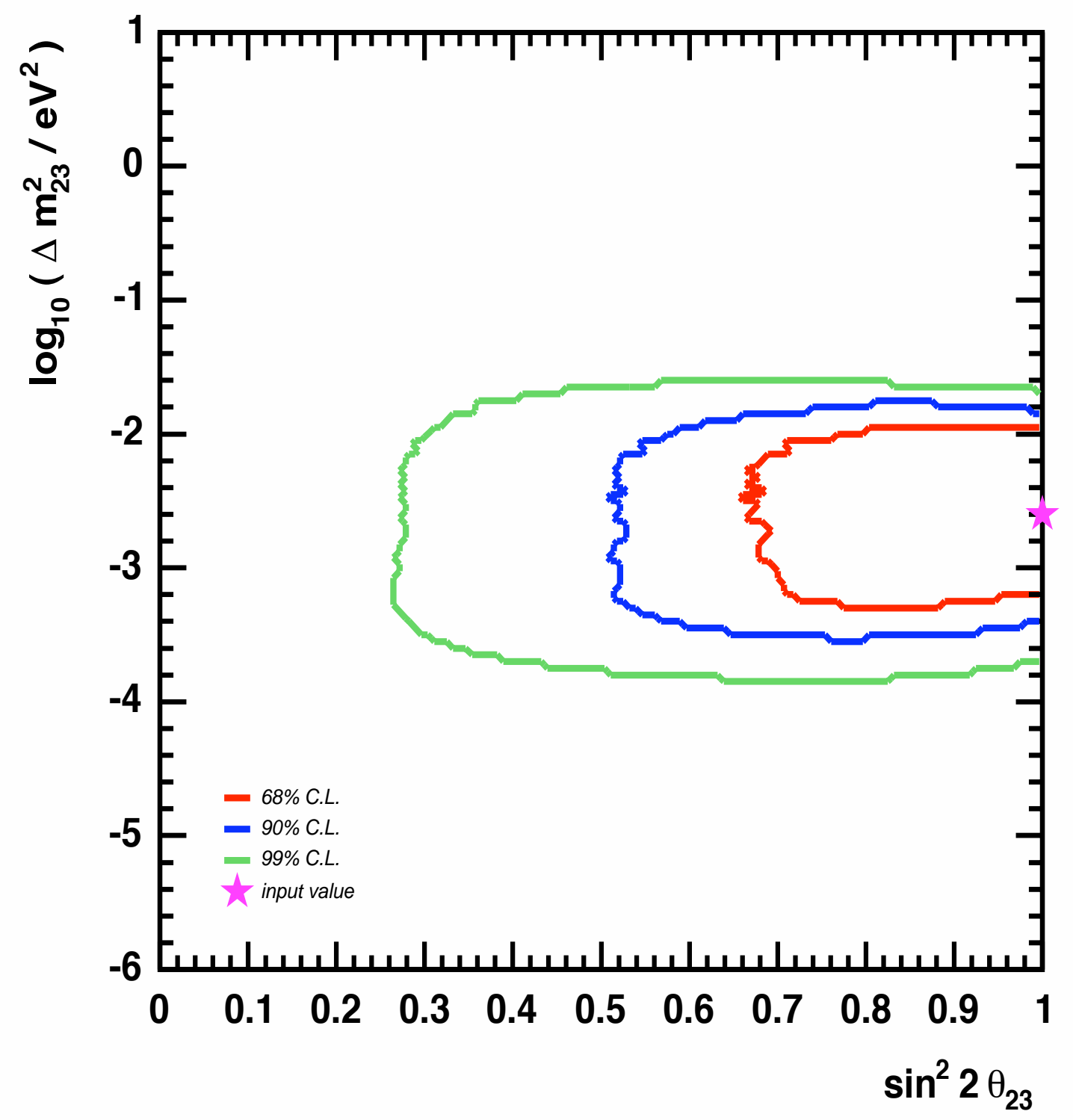

Figure 9.20: Projected confidence limits on oscillation parameters after 25 $k T$-Yrs for input parameters $\Delta m_{23}^{2}=2.5 \times 10^{-3} \mathrm{eV}^{2}, \sin ^{2} 2 \theta_{23}=1.0$. 


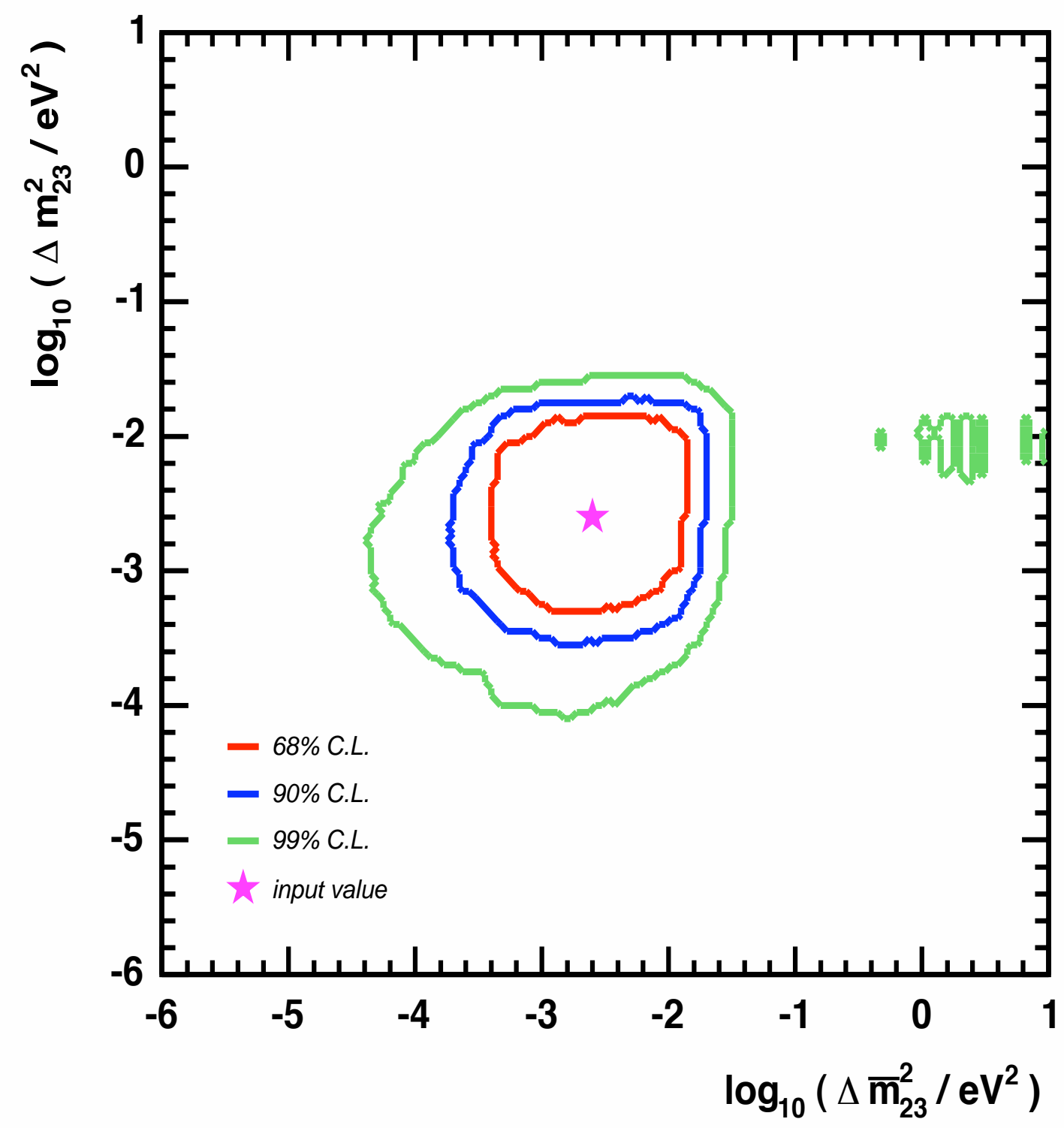

Figure 9.21: Projected confidence limits on oscillation parameters after 25 $k T$-Yrs for input parameters $\Delta m_{23}^{2}=\Delta \bar{m}_{23}^{2}=2.5 \times 10^{-3} e^{2}$ assuming $\sin ^{2} 2 \theta_{23}=\sin ^{2} 2 \bar{\theta}_{23}=1.0$. 

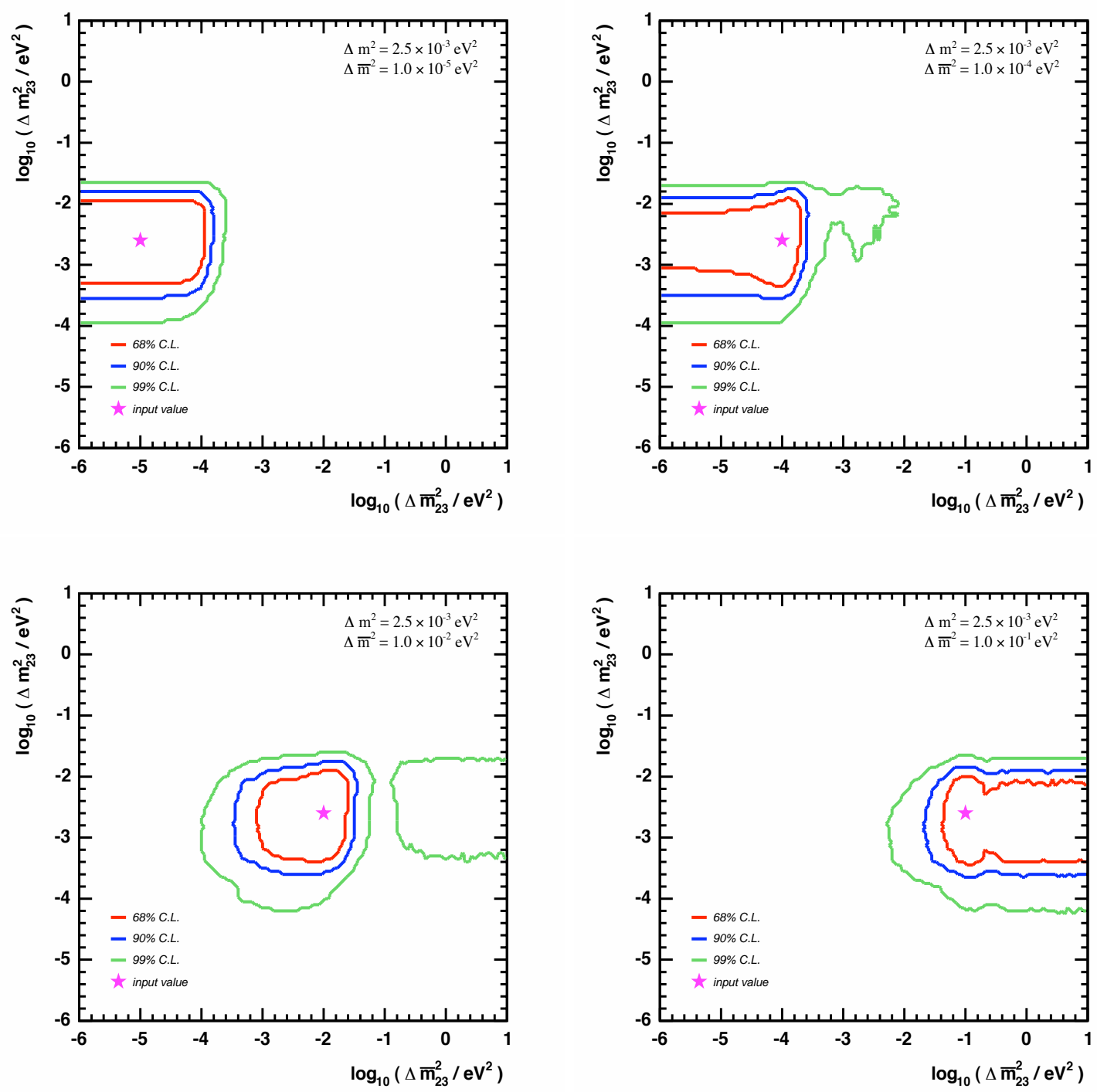

Figure 9.22: Projected confidence limits on oscillation parameters after $25 \mathrm{kT}$-Yrs for input parameters $\Delta m_{23}^{2}=2.5 \times 10^{-3} \mathrm{eV}^{2}, \Delta \bar{m}_{23}^{2}=$ $10^{-5}, 10^{-4}, 10^{-2}, 10^{-1} e^{2}$ assuming $\sin ^{2} 2 \theta_{23}=\sin ^{2} 2 \bar{\theta}_{23}=1.0$. 


\section{Chapter 10}

\section{Conclusion}

The Far Detector has been collecting atmospheric neutrino data since 1st August 2003. The analysis presented here is based on data collected up to 1st October 2004, corresponding to a total detector exposure of 4.67 kT-Yrs. The Far Detector systems are well understood and monitored in detail. A set of well-defined data quality checks are used to select production data for the atmospheric neutrino analysis. The data has been calibrated to correct for variations in timing and response across the detector. Timing information is particularly important for the atmospheric neutrino analysis since it is used to measure the direction of atmospheric neutrino events. The detector timing system has been calibrated at the sub-nanosecond level.

A series of algorithms have been developed to identify and reconstruct atmospheric $\nu_{\mu} / \bar{\nu}_{\mu}$ CC interactions in the Far Detector. The signature of these events is a muon track with a contained interaction vertex. The particle tracks and showers produced by atmospheric neutrino interactions are reconstructed by analysing the topology of events. The track and shower kinematies are reconstructed and are then combined to give the overall neutrino kinematics. The atmospheric $\nu_{\mu} / \bar{\nu}_{\mu} \mathrm{CC}$ signal must be selected from a high background of cosmic muons. In order to separate the signal and background, events are divided into fully contained, down-going partially contained and up-going partially contained event classes. The main background to fully contained and downgoing partially contained atmospheric neutrinos arises from cosmic muons that enter the detector between planes and appear contained. The signal and background are separated by applying a series of containment cuts and then using the veto shield to tag the majority of the remaining cosmic muon background. The main background to 
up-going partially contained atmospheric neutrinos arises from cosmic muons that are mis-reconstructed as up-going from the timing information. The signal and background are separated by applying a series of timing cuts to select well-measured up-going events. The cosmic muon background has been reduced to the level of $<5 \%$.

A total of 82 candidate atmospheric neutrino events are selected from the data. This compares with an expectation of $109.9 \pm 21.4$ events in the absence of neutrino oscillations. The candidate events are separated into up-going and down-going events based on the direction of the muon track. Of the 82 selected events, 60 events have a clearly identified direction with 22 events classified as up-going and 38 events classified as down-going. The ratio between the measured and expected up/down ratios is:

$$
\frac{\left(R_{u / d}\right)_{D A T A}}{\left(R_{u / d}\right)_{M C}}=0.62 \pm 0.17(\text { stat }) \pm 0.04(\text { sys })
$$

This is 2.2 standard deviations away from the expectation for no oscillations.

The candidate events are separated into $\nu_{\mu}$ and $\bar{\nu}_{\mu}$ based on the curvature of the muon track. Of the 60 events with a clearly identified direction, 40 events have a clearly identified charge with 27 events classified as neutrinos and 13 events classified as anti-neutrinos. This represents the first direct observation of atmospheric $\nu_{\mu}$ and $\bar{\nu}_{\mu}$ interactions. The ratio between the measured and expected $\bar{\nu}_{\mu} / \nu_{\mu}$ ratios is:

$$
\frac{\left(R_{\bar{\nu} / \nu}\right)_{\nu A T A}}{\left(R_{\bar{\nu} / \nu}\right)_{M C}}=0.90 \pm 0.29(\text { stat }) \pm 0.06(\text { sys })
$$

The measured and expected ratios are consistent within crrors.

A maximum likelihood analysis is used to determine the oscillation parameters $\left(\Delta m_{23}^{2}, \sin ^{2} \theta_{23}\right)$ assuming two-flavour oscillations. The best fit point is: $\Delta m_{23}^{2}=$ $1.3 \times 10^{-3} \mathrm{cV}^{2}, \sin ^{2} \theta_{23}=0.9$. The data are consistent with a large range of oscillation parameters. The null oscillation hypothesis is disfavoured at the $79 \%$ confidence level. The data are divided into neutrinos and anti-neutrinos and a second fit is applied to detcrmine the oscillation parameters $\left(\Delta m_{23}^{2}, \Delta \bar{m}_{23}^{2}\right)$ assuming $\left(\sin ^{2} \theta_{23}=\sin ^{2} \bar{\theta}_{23}=1.0\right)$. The best fit point is: $\Delta m_{23}^{2}=3.0 \times 10^{-1} \mathrm{eV}^{2}, \Delta \bar{m}_{23}^{2}=6.0 \times 10^{-3} \mathrm{eV}^{2}$. The data are consistent with a large range of oscillation parameters. Assuming neutrino oscillations with $\Delta m_{23}^{2}=2.5 \times 10^{-3} \mathrm{eV}^{2}$, the entire range of anti-neutrino oscillation parameters is found to lie within the $68 \%$ confidence limit. 
The expected sensitivity is calculated for the combined and separated $\nu_{\mu} / \bar{\nu}_{\mu}$ oscillation fits for a detector cxposure of $25 \mathrm{kT}$-Yrs . Assuming ncutrino oscillations with $\Delta m_{23}^{2}=2.5 \times 10^{-3} \mathrm{eV}^{2}$ and $\sin ^{2} \theta_{23}=1.0$, the oscillation parameters are determined to be in the range $4 \times 10^{-4}<\Delta m_{23}^{2}<2 \times 10^{-2} \mathrm{cV}^{2}$, $\sin ^{2} \theta_{23}>0.5$ at the $90 \%$ confidence level and differences of $1-2$ orders of magnitude between $\Delta m_{23}^{2}$ and $\Delta \bar{m}_{23}^{2}$ are resolved. The sensitivity of the analysis can be enhanced by a number of possible improvements in the reconstruction and sclection of events, and in the fitting procedure. The analysis currently only considers atmospheric neutrino interactions contained inside the detector. These events can be combined with up-going muons produced by atmospheric neutrino interactions in the rock to improve the overall sensitivity. The large systematic crror in the absolute atmospheric neutrino flux can also be reduced by using charged current electron neutrino events from the Far Detector or the Soudan 2 experiment to normalize the expected distributions of charged current muon neutrino events. Finally, the $L / E$ resolution can be improved by using the neutrino direction to determine the propagation distance in place of the muon direction. A more accurate reconstruction of the neutrino kinematics is possible by combining the track and shower kinematics with a knowledge of the underlying physics of neutrino interactions.

This analysis has devcloped a method for sclecting a low background sample of atmospheric $\nu_{\mu} / \bar{\nu}_{\mu}$ events and reconstructing the neutrino kinematics. The first separate observation of atmospheric $\nu_{\mu}$ and $\bar{\nu}_{\mu}$ interactions has been performed. With the current statistics, the sensitivity to neutrino oscillations is still limited. However, the atmospheric neutrino analysis will continue over the coming years. 


\section{Appendix A}

\section{Atmospheric Neutrino Events}

The analysis is based on data collected at the Far Detector between 1st August 2003 and 31 st October 2004 (runs in the range $18143-27793$ ). The total livetime is 315.9 days (204.9 days with magnetic ficld forward and 111.0 days with magnetic ficld reversed). The 82 candidate atmospheric $\nu_{\mu} / \bar{\nu}_{\mu}$ CC events are listed in the tables provided below. The tables are separated into FC events, down-going PC events and up-going PC events. The following information is provided in each table:

- Day (defined relative to 1st August 2003).

- Run/Snarl number

- Up-going/Down-going

- Neutrino/Anti-Neutrino

- Muon Momentum

- Shower Energy 


\section{A.1 Fully Contained Events}

\begin{tabular}{|c|c|c|c|c|c|c|c|}
\hline & Day & Run & Snarl & $\mathrm{u} / \mathrm{d}$ & $\nu_{\mu} / \bar{\nu}_{\mu}$ & $\mathrm{E}_{\mu} / \mathrm{GeV}$ & $\mathrm{E}_{s h w} / \mathrm{GeV}$ \\
\hline 1 & 16 & 18581 & 63807 & d & - & 0.7 & 0.2 \\
\hline 2 & 29 & 18866 & 62010 & $\mathrm{~d}$ & - & 2.0 & 0.1 \\
\hline 3 & 71 & 20131 & 33435 & d & $\nu_{\mu}$ & 0.8 & 0.4 \\
\hline 4 & 71 & 20147 & 26192 & - & - & 0.3 & 0.0 \\
\hline 5 & 82 & 20629 & 31711 & $\mathrm{u}$ & $\nu_{\mu}$ & 2.3 & 0.4 \\
\hline 6 & 88 & 20818 & 65032 & $\mathrm{u}$ & $\nu_{\mu}$ & 1.1 & 0.3 \\
\hline 7 & 93 & 20998 & 6938 & $\mathrm{~d}$ & $\nu_{\mu}$ & 3.5 & 4.6 \\
\hline 8 & 123 & 21737 & 63416 & 11 & $\nu_{\mu}$ & 1.2 & 3.6 \\
\hline 9 & 133 & 21906 & 116585 & - & - & 0.7 & 0.0 \\
\hline 10 & $14 j$ & 22165 & 6613 & - & - & 0.5 & 1.2 \\
\hline 11 & 152 & 22236 & 83171 & $\mathrm{u}$ & - & 0.7 & 0.6 \\
\hline 12 & 166 & 22424 & 149574 & - & - & 0.5 & 0.0 \\
\hline 13 & 168 & 22446 & 82423 & $\mathrm{~d}$ & $\nu_{\mu}$ & 3.7 & 0.9 \\
\hline 14 & 173 & 22509 & 24855 & - & - & 0.8 & 0.2 \\
\hline 15 & 177 & 22575 & 95688 & - & - & 0.3 & 0.3 \\
\hline 16 & 189 & 22708 & 84455 & $\mathrm{~d}$ & $\nu_{\mu}$ & 1.4 & 2.9 \\
\hline 17 & 189 & 22713 & 10258 & - & - & 0.6 & 0.1 \\
\hline 18 & 199 & 22825 & 32924 & - & - & 0.5 & 0.6 \\
\hline 19 & 202 & 22850 & 92736 & - & - & 0.7 & 0.2 \\
\hline 20 & 202 & 22868 & 10353 & $d$ & $\nu_{\mu}$ & 0.7 & 0.2 \\
\hline 21 & 206 & 22919 & 7988 & $d$ & $\bar{\nu}_{\mu}$ & 1.9 & 0.2 \\
\hline 22 & 226 & 23285 & 59052 & $\mathrm{u}$ & - & 0.7 & 0.1 \\
\hline 23 & 227 & 23297 & 43710 & 11 & $\nu_{\mu}$ & 1.3 & 0.8 \\
\hline 24 & 230 & 23604 & 95164 & $d$ & - & 0.8 & 1.0 \\
\hline 25 & 235 & 23943 & 85316 & - & - & 0.6 & 0.1 \\
\hline 26 & 243 & 24088 & 8719 & $\mathrm{u}$ & $\nu_{\mu}$ & 0.7 & 0.3 \\
\hline 27 & 252 & 24829 & 87042 & 11 & $\nu_{\mu}$ & 1.0 & $0 . \bar{\jmath}$ \\
\hline
\end{tabular}




\begin{tabular}{|c|c|c|c|c|c|c|c|}
\hline & Day & Run & Snarl & $\mathrm{u} / \mathrm{d}$ & $\nu_{\mu} / \bar{\nu}_{\mu}$ & $\mathrm{E}_{\mu} / \mathrm{GeV}$ & $\mathrm{E}_{s h w} / \mathrm{GeV}$ \\
\hline 28 & 253 & 24844 & 97277 & 11 & $\bar{\nu}_{\mu}$ & 1.7 & 0.3 \\
\hline 29 & 256 & 24871 & 120533 & - & - & 1.0 & 1 \\
\hline 30 & 256 & 24881 & 44348 & - & - & 0.9 & 0.7 \\
\hline 31 & 258 & 24941 & 72237 & $\mathrm{~d}$ & $\nu_{\mu}$ & 1.1 & 1.1 \\
\hline 32 & 262 & 24982 & 23731 & 11 & $\nu_{\mu}$ & 2.7 & 7.2 \\
\hline 33 & 263 & 24988 & 8655 & $d$ & $\bar{\nu}_{\mu}$ & 1.5 & 0.1 \\
\hline 34 & 266 & 25048 & 25582 & $\mathrm{~d}$ & - & 1.3 & 0.3 \\
\hline 35 & 272 & 25106 & 78421 & - & - & 0.4 & 1.0 \\
\hline 36 & 276 & 25142 & 38534 & - & - & 0.6 & 1.4 \\
\hline 37 & 277 & 25154 & 96745 & - & - & 0.6 & 1.5 \\
\hline 38 & 309 & 25589 & 98333 & - & - & 0.6 & 0.4 \\
\hline 39 & 319 & 25689 & 73688 & d & - & 0.7 & 0.3 \\
\hline 40 & 331 & 25831 & 21795 & - & - & 0.4 & 0.1 \\
\hline 41 & 344 & 25988 & 40460 & $d$ & $\nu_{\mu}$ & 2.4 & 0.3 \\
\hline 42 & 345 & 25991 & 67835 & $\mathrm{~d}$ & $\bar{\nu}_{\mu}$ & 0.9 & 0.1 \\
\hline 43 & 345 & 25991 & 68957 & d & $\nu_{\mu}$ & 3.7 & 1.6 \\
\hline 44 & 346 & 26000 & 60501 & - & - & 0.6 & 0.0 \\
\hline $4 j$ & 363 & 26348 & 57497 & - & - & 1.0 & 1.1 \\
\hline 46 & 412 & 27184 & 116702 & $\mathrm{~d}$ & $\nu_{\mu}$ & 0.8 & 0.1 \\
\hline 47 & 419 & 27292 & 47839 & $\mathrm{~d}$ & - & 0.8 & 0.8 \\
\hline 48 & 422 & 27325 & 123806 & $u$ & $\nu_{\mu}$ & 0.8 & 0.8 \\
\hline 49 & 435 & 27566 & 28709 & - & - & $0 . \bar{j}$ & 0.1 \\
\hline 50 & 437 & 27581 & 61928 & d & $\nu_{\mu}$ & 0.8 & 0.2 \\
\hline 51 & 448 & 27694 & 103569 & d & $\nu_{\mu}$ & 1.6 & 0.2 \\
\hline 52 & 450 & 27709 & 19610 & $\mathrm{~d}$ & $\nu_{\mu}$ & 0.7 & 4.2 \\
\hline 53 & 451 & 27721 & 13243 & d & $\bar{\nu}_{\mu}$ & 1.2 & 0.2 \\
\hline 54 & 453 & 27740 & 63058 & 11 & $\nu_{\mu}$ & 0.7 & 0.1 \\
\hline $5 \overline{5}$ & 453 & 27748 & 1328 & d & - & 0.8 & 0.0 \\
\hline 56 & 456 & 27781 & 54144 & - & - & 0.4 & 0.2 \\
\hline
\end{tabular}




\section{A.2 Down-Going Partially Contained Events}

\begin{tabular}{|c|c|c|c|c|c|c|c|}
\hline & Day & Run & Snarl & $\mathbf{u} / \mathbf{d}$ & $\nu_{\mu} / \bar{\nu}_{\mu}$ & $\mathrm{E}_{\mu} / \mathrm{GeV}$ & $\mathbf{E}_{\text {shu }} / \mathrm{GeV}$ \\
\hline 1 & 29 & 18862 & 36180 & - & - & 7.6 & 0.6 \\
\hline 2 & 90 & 20884 & 27361 & $\mathrm{~d}$ & - & 24.9 & 0.0 \\
\hline 3 & 139 & 22050 & 51358 & $\mathrm{~d}$ & - & 43.1 & 3.6 \\
\hline 4 & 141 & 22101 & 121177 & $\mathrm{~d}$ & $\nu_{\mu}$ & 1.4 & 0.2 \\
\hline 5 & 149 & 22201 & 10289 & $\mathrm{~d}$ & - & 25.2 & 0.3 \\
\hline 6 & 166 & 22424 & 136567 & $\mathrm{~d}$ & $\bar{\nu}_{\mu}$ & 2.0 & 0.7 \\
\hline 7 & 206 & 22919 & 67340 & $\mathrm{~d}$ & $\bar{\nu}_{\mu}$ & 5.8 & 1.5 \\
\hline 8 & 226 & 23285 & 33554 & $\mathrm{~d}$ & - & 37.6 & 3.0 \\
\hline 9 & 229 & 23584 & 107525 & $\mathrm{~d}$ & $\nu_{\mu}$ & 1.5 & 1.4 \\
\hline 10 & 251 & 24829 & 36847 & $\mathrm{~d}$ & $\nu_{\mu}$ & 2.7 & 1.6 \\
\hline 11 & 260 & 24964 & 13099 & $\mathrm{~d}$ & $\nu_{\mu}$ & 0.7 & 2.2 \\
\hline 12 & 268 & 25066 & 70672 & $\mathrm{~d}$ & $\bar{\nu}_{\mu}$ & 4.0 & 0.3 \\
\hline 13 & 312 & 25627 & 47998 & $\mathrm{~d}$ & - & 17.7 & 0.4 \\
\hline 14 & 326 & 25773 & 114723 & $\mathrm{~d}$ & $\bar{\nu}_{\mu}$ & 8.4 & 6 \\
\hline 15 & 356 & 26277 & 28086 & $\mathrm{~d}$ & - & 9.4 & 5.8 \\
\hline 16 & 400 & 27045 & 20111 & $\mathrm{~d}$ & - & 13.4 & 8.4 \\
\hline
\end{tabular}




\section{A.3 Up-Going Partially Contained Events}

\begin{tabular}{|c|c|c|c|c|c|c|c|}
\hline & Day & Run & Snarl & $\mathbf{u} / \mathbf{d}$ & $\nu_{\mu} / \bar{\nu}_{\mu}$ & $\mathrm{E}_{\mu} / \mathrm{GeV}$ & $\mathrm{E}_{s h w} / \mathrm{GeV}$ \\
\hline 1 & 31 & 18902 & 36351 & $u$ & - & 20.6 & 1.7 \\
\hline 2 & 85 & 20747 & 17532 & $\mathrm{u}$ & - & 2.9 & 2.2 \\
\hline 3 & 124 & 21772 & 49880 & $\mathrm{u}$ & - & 3.2 & 2.0 \\
\hline 4 & 128 & 21853 & 24825 & $\mathrm{u}$ & $\bar{\nu}_{\mu}$ & 2.1 & 0.8 \\
\hline 5 & 161 & 22336 & 22507 & $\mathrm{u}$ & $\nu_{\mu}$ & 2.4 & 0.2 \\
\hline 6 & 256 & 24874 & 37215 & $\mathrm{u}$ & $\bar{\nu}_{\mu}$ & 4.4 & 0.3 \\
\hline 7 & 278 & 25189 & 77584 & $\mathrm{u}$ & - & 8.4 & 2.2 \\
\hline 8 & 345 & 25988 & 89674 & $\mathrm{u}$ & $\bar{\nu}_{\mu}$ & 4.6 & 0.4 \\
\hline 9 & 381 & 26830 & 27336 & $\mathrm{u}$ & $\nu_{\mu}$ & 1.1 & 4.5 \\
\hline 10 & 452 & 27724 & 85333 & $\mathrm{u}$ & $\bar{\nu}_{\mu}$ & 7.1 & 1.1 \\
\hline
\end{tabular}




\section{Bibliography}

[1] Fermi E, An attempt of a theory of beta radiation, Z. Phys. 88, 161-177 (1934).

[2] Cowan C. et al, Detection of the free neutrino: a confirmation, Science 124, 103-4 (1956).

[3] Danby G. et al, Obscrvation of high encrgy neutrino reactions and the cxistence of two kinds of neutrinos, Phys. Rev. Lett. 9, 36-44 (1962).

[4] Perl M. et al, Evidence for anomalous lepton production in $e^{+}-e^{-}$annihilation, Phys Rev Lett. 35, 1489-1492 (1975).

[5] Innes W. et al, Observation of structure in the $\Upsilon$ region, Phys. Rev. Lett. 39, 12402 (1977).

[6] (The DONUT collaboration) Kodama K. ct al, Obscrvation of taul-ncutrino interactions, Phys. Lett. B504, 218-224 (2001).

[7] The LEP collaboration, A combination of preliminary electroweak measurements and constraints on the Standard Model, hep-ex/0412015 (2004).

[8] (The Mainz collaboration) Kraus C. et al, Final results from phase II of the Mainz ncutrino mass scarch in tritium $\beta$ decay, Eur. Phys. J. 40, 447468 (2005).

[9] Lobashev V, Direct search for the neutrino mass in the beta decay of tritium: Status of the Troitsk nu-mass experiment, Phys. Atom. Nucl. 63, 962-968 (2000).

[10] Assamagan K. et al, Lpper limit of the muon-neutrino mass and charged pion mass from momentum analysis of a surface muon beam, Phys. Rev. D53, 606j-6077 (1996). 
[11] (The ALEPH collaboration) Barate R. et al, An upper limit on the tau neutrino mass from threc- and fivc-prong tau decays, Eur. Phys. J. C2, 395406 (1998).

[12] Sanchez A. et al, Cosmological parameters from CMB measurements and the final 2dFGRS power spectrum, astro-ph/0507583 (2005).

[13] Tegmark M. et al, Cosmological parameters from SDSS and WMAP, Phys. Rev. D69, 103501 (2004).

[14] (The SNO collaboration) Ahmad Q. et al, Measurement of the rate of $\nu_{e}+d \rightarrow$ $p+p+e^{-}$interactions produced by ${ }^{8} \mathrm{~B}$ solar neutrinos at the Sudbury Neutrino Obscrvatory, Phys. Rev. Lett. 87, 071301 (2001).

[15] (The Super-Kamiokande collaboration) Fukuda Y. et al, Evidence for oscillation of atmospheric neutrinos, Phys. Rev. Lett. 81, 1562-7 (1998).

[16] (The Soudan 2 collaboration) Sanchez M. et al, Observation of atmospheric neutrino oscillations in Soudan 2, Phys. Rev. D68, 113004 (2003).

[17] (The MACRO collaboration) Ambrosio M. et al, Atmospheric neutrino oscillations from upward through-going muon multiple scattering in MACRO, Phys. Lett. B566, 3544 (2003).

[18] (The KamLAND collaboration) Eguchi K. et al, First results from KamLAND: Evidence for reactor anti-neutrino disappearance, Phys. Rev. Lett. 90, 021802 (2003).

[19] (The K2K collaboration) Ahn M. ct al, Indications of ncutrino oscillation in a 250 km long-baseline experiment, Phys. Rev. Lett. 90, 041801 (2003).

[20] Maki Z. Nakagawa M. Sakata S, Remarks on the unified model of elementary particles, Prog. Theor. Phys. 28, 870 (1962).

[21] Pontecorvo B, Sov. Phys. JETP 53, 1717 (1967).

[22] Bahcall J. Serenelli A. Basu S, New solar opacities, abundances, helioseismology, and neutrino fluxes, Astrophys. J. 621, L85-8 (2005).

[23] Davis R. Harmer D. Hoffman K, Search for neutrinos from the sun, Phys. Rev. Lett. 20, 1205-9 (1968). 
[24] Cleveland B. et al, Measurement of the solar electron neutrino flux with the Homestake chlorine detector, Astrophys. J. 496, 505526 (1998).

[25] (The SAGE collaboration) Abazov A. et al, Search for neutrinos from sun using the reaction ${ }^{71} \mathrm{Ga}\left(\nu_{e}, e^{-}\right)^{71} \mathrm{Gc}$, Phys. Rev. Lett. 67, 33325 (1991).

[26] (The GALLEX collaboration) Anselmann P. et al, Solar neutrinos observed by GALLEX at Gran Sasso, Phys. Lett. B285, 376-389 (1992).

[27] (The SAGE collaboration) Abdurashitov J. et al, Measurement of the solar neutrino capture rate by the Russian-American gallium solar neutrino experiment during one half of the 22-year cycle of solar activity, J. Exp. Theor. Phys. 95, 181-193 (2002).

[28] (The GALLEX collaboration) Hampela W. et al, GALLEX solar neutrino observations: results for GALLEX IV, Phys. Lett. B447, 127133 (1999).

[29] Bahcall J. Basu S. Pinsonneault M, How uncertain are solar neutrino predictions?, Phys. Lett. B433, 1-8 (1998).

[30] (The Kamiokande collaboration) Hirata K. et al, Obscrvation of B-8 solar neutrinos in the Kamiokande-II detector, Phys. Rev. Lett. 63, 16-19 (1989).

[31] (The Kamiokande collaboration) Fukuda Y. ct al, Solar neutrino data covcring solar cycle 22, Phys. Rev. Lett. 77, 1683-6 (1996).

[32] (The Super-Kamiokande collaboration) Fukuda S. et al, The Super-Kamiokande detector, Nucl. Instr. Meth. A501, 418462 (2003).

[33] (The Super-Kamiokande collaboration) Fukuda S. et al, Solar B-8 and hep neutrino measurements from 1258 days of Super-Kamiokande data, Phys. Rev. Lett. 86, $5651-5$ (2001).

[34] (The Super-Kamiokande collaboration) Fukuda S. et al, Determination of solar neutrino oscillation parameters 11sing 1496 days of Supcr-Kamiokande I data, Phys. Lett. B539, 179-187 (2002).

[35] (The SNO collaboration) Boger J. et al, The Sudbury Neutrino Observatory, Nucl. Instr. Meth. A449, 172-207 (2000). 
[36] (The SNO collaboration) Aharmim B. et al, Electron energy spectra, fluxes, and day-night asymmetrics of ${ }^{8} \mathrm{~B}$ solar neutrinos from the 391-day salt phase SNO data set, nucl-ex/0502021 (2005).

[37] Mikheev S. Smirnov A, Resonance enhancement of oscillations in matter and solar neutrino spectroscopy; Sov. J. Nucl. Phys. 42, 913-917 (1985).

[38] Wolfenstcin L, Neutrino oscillations in matter, Phys. Rev. D17, 23692374 (1978).

[39] Shirai J, Start of KamLAND, Nucl. Phys. Proc. Suppl. 118, 15-22 (2003).

[40] (The KamLAND collaboration) Araki T. et al, Measurement of neutrino oscillation with KamLA.ND: evidence of spectral distortion, Phys. Rev. Lett. 94, 081801 (2005).

[41] (The IMB collaboration) Becker-Szendy R. et al, I.MB-3: A large water cerenkov detector for nucloon decay and neutrino interactions, Nucl. Instr. Meth. A324, 363-382 (199).

[42] (The Kamiokande collaboration) Hirata K. et al, Expcrimental study of the atmospheric neutrino flux, Phys. Lett. B205, 416 (1988).

[43] (The IMB collaboration) Becker-Szendy R. et al, The clectron-neutrino and muonneutrino content of the atmospheric flux, Phys. Rev. D46, 3720-4 (1992).

[44] (The Kamiokande collaboration) Hirata K. ct al, Observation of a small atmospheric $\nu_{\mu} / \nu_{e}$ ratio in Kamiokande, Phys. Lett. B280, 146-152 (1992).

[45] (The NUSEX collaboration) Battistoni G. et al, The NUSEX detector, Nucl. Instr. Meth. A245, 277-290 (1986).

[46] (The NUSEX collaboration) Aglictta M. ct al., Expcrimental study of atmospheric neutrino flux in the NLSEX experiment, Europhys. Lett. 8, 611-614 (1989).

[47] (The Frejus collaboration) Berger C. et al, The Frejus nucleon decay detector, Nucl. Instr. Meth. A262, 463-495 (1987).

[48] (The Frejus collaboration) Berger C. et al, A study of neutrino oscillations in the Frejus experiment, Phys. Lett. B245, 305-310 (1990). 
[49] (The Soudan 2 collaboration) Thron J. et al, The Soudan II proton deacy experiment, Nucl. Instr. Meth A283, 6425 (1989).

[j0] (The Soudan 2 collaboration) Allison W. et al, Measurement of the atmospheric ncutrino flavour composition in Soudan 2, Phys. Lett. B391, 491500 (1997).

[51] (The Super-Kamiokande collaboration) Ashie Y. et al, A measurement of atmospheric neutrino oscillation parameters by Super-Kamiokande I, Phys. Rev. D71, $112005(2005)$.

[52] Ashie Y. et al, Evidence for an oscillatory signature in atmospheric neutrino oscillation, Phys. Rev. Lett. 99, 101801 (2004).

[53] Barger V. et al, Neutrino decay as an explanation of atmospheric neutrino observations, Phys. Rev. Lett. 82, 2640-2643 (1999).

[54] Barger V. et al, Neutrino decay and atmospheric neutrinos, Phys. Lett. B462, 109-114 (1999).

[55] Lisi E. Marrone A. Montanino D, Probing possible decoherence effects in atmospheric neutrino oscillations, Phys. Rev. Lett. 85, 11669 (2000).

[j6] Fogli G. Lisi E. Marrone A. Montanino D, Status of atmospheric neutrino $\nu_{\mu} \leftrightarrow \nu_{\tau}$ oscillations and decoherence after the first K2K spectral data, Phys. Rev. D67, 093006 (2003).

[57] (The K2K collaboration) Ahn S. et al, Detection of accelerator produced neutrinos at a distance of $250 \mathrm{~km}$, Phys. Lett. B511, 178184 (2001).

[58] (The K2K collaboration) Aliu E. et al, Evidence for muon neutrino oscillation in an accclcrator-based cxpcriment, Phys. Rev. Lett. 94, 081802 (2005).

[59] (The CHOOZ collaboration) Apollonio M. et al, Initial results from the CHOOZ long baseline reactor neutrino oscillation experiment, Phys. Lett. B420, 397-404 (1998).

[60] (The CHOOZ collaboration) Apollonio M. et al, Search for neutrino oscillations on a long base-line at the CHOOZ nuclear power station, Eur. Phys. J. C27, $331-374(2003)$. 
[61] (The LSND collaboration) Athanassopoulos C. et al, The Liquid Scintillator Neutrino Detector and LAMPF neutrino source, Nucl. Instr. Meth. A388, 149172 (1997).

[62] (The LSND collaboration) Aguilar A. ct al, Evidence for neutrino oscillations from the observation of $\bar{\nu}_{e}$ appearance in a $\bar{\nu}_{\mu}$ beam, Phys. Rev. D64, 112007 $(2001)$.

[63] (The KARMEN collaboration) Armbruster B. ct al, Upper limits for neutrino oscillations $\bar{\nu}_{\mu} \rightarrow \bar{\nu}_{e}$ from muon decay at rest, Phys. Rev. D65, 112001 (2002).

[64] (The BLGEY collaboration) Declais Y. et al, Search for neutrino oscillations at 15-meters, 40-meters, and 95-meters from a nuclear power reactor at Bugey, Nucl. Phys. B434, ј03-534 (1995).

[65] Strumia A, Interpreting the LSND anomaly: stcrile neutrinos or CPT-violation or...?, Phys. Lett. B539, 91-101 (2002).

[66] Barenboim G. Borissov L. Lykken J, Neutrinos that violate CPT, and the experiments that love them, Phys. Lett. B534, 106113 (2002).

[67] (The BooNE collaboartion) Church, E. et al, A letter of intent for an experiment to measure $\nu_{\mu} \rightarrow \nu_{e}$ oscillations and $\nu_{\mu}$ disappearance at the Fermilab booster: BooNE, nucl-ex/9706011 (1997).

[68] Fukugita M. Yanagida T, Baryogenesis without grand unification, Phys. Lett. B175, 457 (1986).

[69] Buchmuller W. Peccei R. Yanagida P, Leptogenesis as the origin of matter, hep$p h / 0502169(2005)$.

[70] (The MINOS collaboration) Adamson P. et al, MINOS Technical Design Report, NUMI-NOTE-GEN-397 (MINOS technical note) (1998).

[71] Litchficld P, Atmospheric neutrinos in MINOS, Nucl. Instr. Meth. A451, 187191 $(2000)$.

[72] Howcroft C, Atmospheric neutrinos in the MINOS Far Detector, PhD Thesis, University of Cambridge (2004). 
[73] Kopp S, The NuMI neutrino beam at Fermilab, physics/0508001 (2005).

[74] Indurthy. D ct al, Ion chambers for monitoring the NuMI neutrino beam at Fermilab, NuMI-CONF-BEAM-1028 (MINOS technical note) (2004).

[75] Drake G. Dawson J. Nelson C, Overview of the Front End Electronics for the Near Detector, NuMI-NOTE-ELEC-0628 (MINOS technical note) (1999).

[76] Diwan M. et al, A study of $\nu_{\mu} \rightarrow \nu_{e}$ sensitivity in MINOS, NuMI-NOTE-SIM-714 (MINOS technical note) (2001).

[77] Arroyo C, Simulation of MINOS CC analysis, NuMI-NOTE-SIM-486 (MINOS technical note) (1999).

[78] (The MINOS Collaboration), Proposal for a five ycar run plan for MINOS, $N u M I$ NOTE-GEN-930 (MINOS technical note) (2003).

[79] Andreopoulos C. et al, Results from the MINOS Far Detector 4-plane prototype, NuMI-ANA-994 (MINOS technical note) (2003).

[80] Hatcher R, A comparison of the gminos simulation to prototype module data, NuMI-NOTE-SIM-478 (MINOS technical note) (1999).

[81] Lang K. et al, Characterization of 1,600 Hamamatsu 16-anode photomultipliers for the MINOS Far Detector, Nucl. Instr. Meth. (in press) (2004).

[82] Avvakumov S. ct al, Spontancous light cmission from fibres in MINOS, Nucl. Instr. Meth. (in press) (2004).

[83] Oliver J. et al, Design and performance of the readout system for the MINOS Far Detector, IEEE Trans. Nucl. Sci. 51, 21935 (2003).

[84] Belias A. et al, The MINOS Data Acquisition system, IEEE Trans. Nucl. Sci. $\mathbf{5 1}, 451-5(2004)$.

[85] Beall E, Documentation of MINOS Detector Control Systems: Design and implementation, NuMI-NOTE-GEN-1089 (MINOS technical note) (2005).

[86] The MINOS collaboration, Proposal for a cosmic ray veto shield for MINOS, NuMI-NOTE-GEN-1037 (MINOS technical note) (2004). 
[87] Adamson P. et al, The MINOS Light Injection calibration system, Nucl. Instr. Meth. A492, 325343 (2002).

[88] Felt $\mathrm{N}$. et al, Far Detector front end electronics documentation, NuMI-NOTEFDDOCS-901 (MINOS technical note) (2003).

[89] Hartnell J, Measurement of the calorimetric energy scale in MINOS, PhD Thesis, University of Oxford (200ॅ).

[90] Nichol R, Calibration of the MINOS detectors, PhD Thesis, University College London (2003).

[91] Smith C, Calibration of the MINOS detectors and extraction of neutrino oscillation parametcrs, PhD Thesis, University College London (2002).

[92] Adamson P. et al, On the linearity of the MINOS Light Injection calibration systcm, Nucl. Instr. Meth. A521, 361366 (2004).

[93] Nichol R, Light Injection calibration at the Calibration Detector, NuMI-NOTEGEN-0974 (MINOS technical note) (2003).

[94] Tagg N, Calibration working group report, talks at the March 2005 MINOS collaboration mecting at Argonne, NuMI-TRANS-GEN-1079 (MINOS technical note) (2005).

[95] Nichol R. Smith C, Strip-to-strip calibration at the Calibration Detector, NuMINOTE-GEN-0972 (MINOS technical note) (2003).

[96] Kordosky M, Hadronic interactions in the MINOS detectors, PhD Thesis, University of Texas (2004).

[97] Vahle P, Electromagnetic interactions in the MINOS detectors, PhD Thesis, University of Texas (2004).

[98] Hatcher R, Active detector (FLS) simulation in gminos and reco-minos, NuMINOTE-SIM-0479 (MINOS technical note) (1999).

[99] Giani S. et al, GEANT detector description and simulation tool, CERN Program Library Long Writeup W5013 (1994). 
[100] Tagg N, Detsim and PhotonTransport: MINOS detector and front-end simulation http://www-numi.fnal.gov/offlinc_software/srt_public_context/wcbdocs/detsim/ detsim_documentation.html.

[101] Birks J, Theory and practice of scintillation counting, (Pergamon Press) (1964).

[102] Kordosky M. et al, M16 response and cross talk simulation, NuMI-NOTE-SIM1044 (MINOS technical note) (2004).

[103] Simpson J, Elcmental and isotropic composition of the galactic cosmic rays, Ann. Rev. Nucl. Part. Sci. 33, 323-381 (1983).

[104] Wang J. et al, Measurement of cosmic-ray hydrogen, helium, and their isotopic composition with the bess experiment, Astrophys. J. 564, 244-259 (2002).

[105] Eidclman S. ct al, Revicw of particle physics, Phys. Lett. B592 (2004).

[106] Boczio M. et al, The cosmic ray proton and helium spectra between 0.4 and 200 $\mathrm{GeV}$, Astrophys. J. 518, 457-472 (1999).

[107] Menn W. et al, The absolute flux of protons and helium at the top of the atmosphere using IMAX, Astrophys. J. 533, 281-297 (2000).

[108] Sanuki T. et al, Precise measurement of cosmic-ray proton and helium spectra with the BESS spectrometer, Astrophys. J. 545, 113542 (2000).

[109] (The AMS collaboration) Alcaraz J. et al, Cosmic protons, Phys. Lett. B490, $27-3 j(2000)$.

[110] Takeda M. et al, Extension of the cosmic-ray energy spectrum beyond the predicted Grcisen-Zatsepin-K11z'min cutoff, Phys. Rev. Lett. 81, 11636 (1998).

[111] Glushkov A. et al, Encrgy spectrum of primary cosmic rays in the energy region of $10^{17}$ to $10^{20} \mathrm{eV}$ by Yakutsk Array data, Proc. 28th ICRC (international cosmic ray conference) , 389-392 (2003).

[112] Bird D. et al, The cosmic ray energy spectrum observed by the Fly's Eye, Astrophys. J. 424, 491-502 (1994). 
[113] (High Resolution Fly's Eye) Abu-Zayyad T. et al, Measurement of the spectrum of UHE cosmic rays by the FADC detector of the HiRes Experiment, astroph/0208301 (2002).

[114] Sigl G, The enigma of the highest energy particles of nature, Annals Phys. 303, $117-141$ (2003).

[115] Kremer J. et al, Mcasurements of ground-level muons at two geomagnetic locations, Phys. Rev. Lett. 83, 4241-4244 (1999).

[116] Motoki M. et al, Precise measurements of atmospheric muon fluxes with the BESS spectrometer, Astropart. Phys. 19, 113-126 (2003).

[117] (The L3 collaboration) Achard P. et al, Measurement of the atmospheric muon spectrum from 20 to $3000 \mathrm{GeV}$, Phys. Lett. B598, 15-32 (2004).

[118] Rebel B, Neutrino-induced muons in the MINOS Far Detector, PhD Thesis, Univerity of Indiana (2004).

[119] Gaisser T, Cosmic rays and particle physics, (Cambridge University Press) (1990).

[120] Kasahara S, A study of cosmic ray composition in the knee region using multiple muon events in the Soudan 2 detector, PhD Thesis, University of Minnesota (1997).

[121] Kasahara S, www.hep.umn.edu/ schubert/far/.

[122] Howeroft C, Atmospheric neutrinos: Contained CC event update, NuMI-TRANSGEN-1004 (MINOS technical note) (2004).

[123] Gaisscr T. Honda M, Flux of atmospheric ncutrinos, Ann. Rev. Nucl. Part. Sci 52, 153-199 (2002).

[124] Barr G. et al., A three-dimensional calculation of atmospheric neutrinos, Phys. Rev. D70, 023006 (2004).

[125] Simpson J, The latitude dependence of neutron densities in the atmosphere as a function of altitude, Phys. Rev. 73, 1389-1391 (1948). 
[126] CLIMAX, http://ulysses.sr.unh.cdu/Neutron.Monitor/Misc/neutron2.html.

[127] Ward C.P, Atmospheric neutrino event rates http://www.hop.phy.cam.ac.11k/ cpw1/flux.pdf.

[128] Engel R. et al, Hadronic interactions for atmospheric cascades, Proc 27th ICRC (international cosmic ray conference), 1318-21 (2001).

[129] Cho Y. et al, Pion production in proton-beryllium collisions at $12.4 \mathrm{GeV} / \mathrm{c}$, Phys. Rev. D4, 1967-1974 (1971).

[130] Eichten et al, Particle production in proton interactions in nuclei at $24 \mathrm{GcV} / \mathrm{c}$, Nucl. Phys. B44, 333-343 (1972).

[131] Abbott T. et al, Mcasurement of particle production in proton-induced reactions at $14.6 \mathrm{GeV} / \mathrm{c}$, Phys. Rev. D45, 3906-3920 (1992).

[132] (The NA49 collaboration) Barr G. et al, Hadroproduction in proton carbon collisions at the NA49 expcriment, Proe 28th ICRC, 15514 (2003).

[133] (The HARP collaboration) Prior G. et al, The HARP experiment: first physics results, Nucl. Phys. A752, 2433 (2005).

[134] Barr G, Atmospheric neutrino fluxes, Nucl. Phys. Proc. Suppl. 143, 89-95 (2005).

[135] Gaisser T, Atmospheric neutrino fluxes, astro-ph/0502380 (2005).

[136] Stanev T, Atmospheric neutrino challenges, atro-ph/0412395 (2004).

[137] Gallagher H, The NEUGEN neutrino event generator, Nucl. Phys. B112, 188-192 (2002).

[138] Merenyi R. et al, Determination of pion intranuclear rescattering rates in $\nu_{\mu}-N e$ versus $\nu_{\mu}-D$ interactions for the atmospheric neutrino flux, Phys. Rev. D45, 743-751 (1992).

[139] Zeller G, Low energy neutrino cross-sections: comparison of various Monte Carlo predictions to experimental data, hep-ex/0312061 (2003). 
[140] Zeitnitz C. Gabriel T, The GEANT-CALOR interface and benchmark calculations of ZEUS test calorimeters, Nucl. Instr. Meth. A349, 106111 (1994).

[141] Zcitnitz C, GCALOR simulation package http://www.staff.uni-mainz.de/zeitnitz/gcalor/gcalor.html.

[142] Battistoni G. et al, The FLUKA atmospheric neutrino flux calculation, Astropart. Phys. 19, 269290 (2003).

[143] Fescfeldt H, GHEISHA - the simulation of hadronic showers: physics and applications; (1985).

[144] Ward C.P, Simulation of neutron background to atmospheric neutrino analyses, NUMI-NOTE-SIM,ATM-NU-1085 (2005).

[145] Thomson M, Alternative demuxer: AltDeMux http://www.hep.phy.cam.ac.uk/ thomson/minos/talks/altdemux.pdf.

[146] Blake A, Neutrino event reconstruction http://www.hep.phy.cam.ac.uk/ blake/fermi03_reco.ppt.

[147] Agostinelli S. et al, GEANT4: A simulation toolkit, Nucl. Instr. Meth. A506, 250 (2003).

[148] Casper D, Current knowledge of neutrino cross-sections and future prospects, $N u$ Fact0 4 ( $6^{\text {th }}$ interntaional workshop on neutrino factories and superbeams) (2004).

[149] Thomson M, Atmospheric neutrino results, NuMI-TRANS-GEN-1095 (MINOS technical note) (2005).

[150] (The Soudan 2 collaboration) Allison W. ct al, Neutrino oscillation effects in Soudan-2 upward-stopping muons, hep-ex/050706 (2005). 